FORSCHUNGSERGEBNISSE DER WIRTSCHAFTSUNIVERSITÄT WIEN

Astrid Haider

\title{
Die Lohnhöhe und Lohnstreuung im Nonprofit-Sektor
}

Eine quantitative Analyse anhand österreichischer Arbeitnehmer-ArbeitgeberDaten 


\section{Astrid Haider}

\section{Die Lohnhöhe und Lohnstreuung im Nonprofit- Sektor}

Über die Lohnstruktur im Nonprofit Sektor ist wenig bekannt. Mit österreichischen Arbeitnehmer-Arbeitgeber-Daten zeigt die Verfasserin, ob und wie ehrenamtliche Mitarbeiter/-innen, Spenden und Subventionen die Lohnhöhe und die innerbetriebliche Lohnstreuung von bezahlten Arbeitnehmer/-innen einer Nonprofit-Organisationen (NPO) beeinflussen. Mithilfe von OLS- und Quantilsregressionen sowie einer Instrumentvariablenschätzung wird gezeigt, dass die Anwesenheit ehrenamtlicher Mitarbeiter/-innen die Lohnhöhe von bezahlten Arbeitskräften senkt. Ein ausgeglichenes zahlenmäßiges Verhältnis von bezahlten und unbezahlten Arbeitskräften reduziert die innerbetriebliche Lohnstreuung. Spenden erhöhen die Lohnhöhe für die oberen Lohngruppen einer NPO, öffentliche Subventionen erhöhen hingegen die Löhne aller Lohngruppen. Sowohl Spenden als auch öffentliche Subventionen vergrößern also die Lohnstreuung in einer NPO.

Astrid Haider, Dr.rer.soc.oec.; Studium der Handelswissenschaft und Soziologie in Wien und Berlin; Doktoratsstudium am Institut für Sozialpolitik an der Wirtschaftsuniversität Wien. 
Die Lohnhöhe und Lohnstreuung im Nonprofit-Sektor 


\section{Forschungsergebnisse der Wirtschaftsuniversität Wien}

Band 39

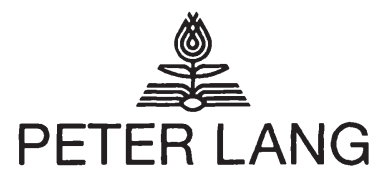

Frankfurt am Main · Berlin · Bern · Bruxelles · New York · Oxford · Wien Astrid Haider - 978-3-631-75377-4 
Astrid Haider

\section{Die Lohnhöhe und Lohnstreuung im Nonprofit-Sektor}

Eine quantitative Analyse anhand österreichischer Arbeitnehmer-Arbeitgeber-Daten

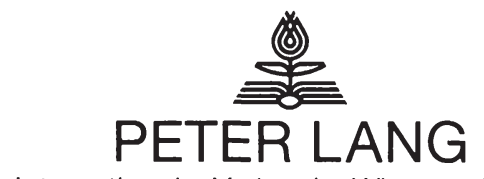

Internationaler Verlag der Wissenschaften

Astrid Haider - 978-3-631-75377-4

Downloaded from PubFactory at 01/11/2019 05:43:53AM

via free access 
Bibliografische Information der Deutschen Nationalbibliothek Die Deutsche Nationalbibliothek verzeichnet diese Publikation in der Deutschen Nationalbibliografie; detaillierte bibliografische Daten sind im Internet über http://dnb.d-nb.de abrufbar.

Open Access: The online version of this publication is published on www.peterlang.com and www.econstor.eu under the international Creative Commons License CC-BY 4.0. Learn more on how you can use and share this work: http://creativecommons.org/ licenses/by/4.0.

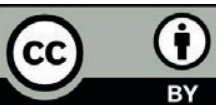

This book is available Open Access thanks to the kind support of ZBW - Leibniz-Informationszentrum Wirtschaft.

Gefördert durch die Wirtschaftsuniversität Wien.

\author{
Umschlaggestaltung: \\ Atelier Platen, nach einem Entwurf \\ der Werbeagentur Publique. \\ Universitätslogo der Wirtschaftsuniversität Wien: \\ Abdruck mit freundlicher Genehmigung \\ der Wirtschaftsuniversität Wien. \\ Gedruckt auf alterungsbeständigem, \\ säurefreiem Papier.
}

ISSN 1613-3056

ISBN 978-3-631-59370-7

ISBN 978-3-631-75377-4 (eBook)

(C) Peter Lang GmbH

Internationaler Verlag der Wissenschaften

Frankfurt am Main 2010

Alle Rechte vorbehalten.

Das Werk einschließlich aller seiner Teile ist urheberrechtlich geschützt. Jede Verwertung außerhalb der engen Grenzen des

Urheberrechtsgesetzes ist ohne Zustimmung des Verlages

unzulässig und strafbar. Das gilt insbesondere für

Vervielfältigungen, Übersetzungen, Mikroverfilmungen und die Einspeicherung und Verarbeitung in elektronischen Systemen.

www.peterlang.de

Astrid Haider - 978-3-631-75377-4 


\section{Vorwort}

Diese Dissertation ist durch zahlreiche Anregungen und Kommentare von Kolleg/-inn/en und Freunden bereichert worden.

In allererster Linie bedanke ich mich bei Prof. Dr. Ulrike Schneider für zahlreiche Anregungen, schnelles Feedback und für guten Rat, wenn ich nicht weiter wusste.

Ich bedanke mich weiterhin bei Prof. Dr. Herbert Walther für die Übernahme der Zweitbetreuung.

Meinen Kolleginnen und Kollegen am Institut für Sozialpolitik danke ich für diverse Diskussionsbeiträge und Hilfestellungen, sowie Motivation - nicht zuletzt durch so manche gemeinsame Kaffeepause.

Ich möchte auch den Mitarbeitern der Statistik Austria danken: Herrn Mag. Robert Leisch als meinen ,ersten Ansprechpartner bei der Statistik Austria“, der für meine Vielzahl an Fragen jederzeit ein offenes Ohr hatte, Herrn Mag. Klaus Stöger, der ebenso wesentlich an der Befragung der Nonprofit Organisationen mitwirkte. Herrn Mag. Reinhold Schwarzl und Herrn Dipl.-Ing.Dr. Johannes Biricz danke ich dafür, dass sie mir ermöglichten, die Einkommenssteuerdaten an der Statistik Austria zu nutzen.

Meiner Familie sei ebenfalls ein ,Dankeschön' dargebracht, vor allem dafür, dass sie für mich eine „dissertationsfreie Zone“ war und ist. Nicht zuletzt bedanke ich mich bei meinem Freund, Dieter Pennerstorfer, fürs unermüdliche Zuhören, Durchlesen, Kommentieren, Mitdenken und Motivieren!

Astrid Haider

September 2008 
Astrid Haider - 978-3-631-75377-4

Downloaded from PubFactory at 01/11/2019 05:43:53AM

via free access 


\section{Inhaltsverzeichnis}

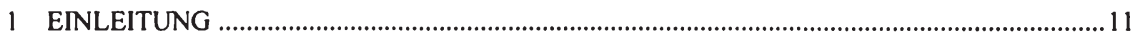

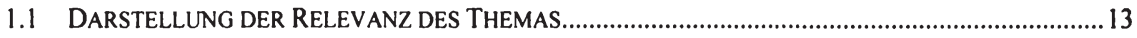

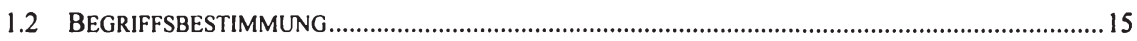

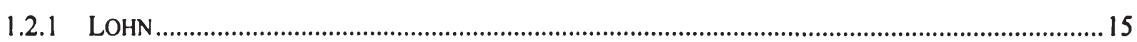

1.2.2 NONPROFIT ORGANISATIONEN UND DER NONPROFIT SEKTOR ................................................16

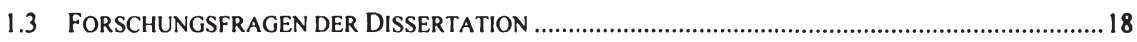

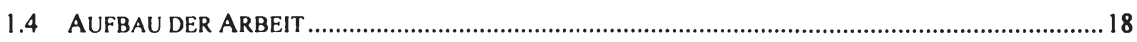

TEIL I: DETERMINANTEN DER LOHNHÖHE VON BEZAHLTEN ARBEITNEHMER/-INNEN

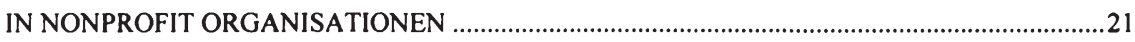



3 ALLGEMEINE THEORIEN ZUR BESTIMMUNG DER LOHNHÖHE ...................................25

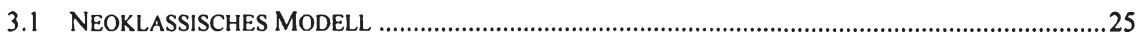

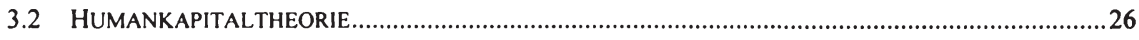

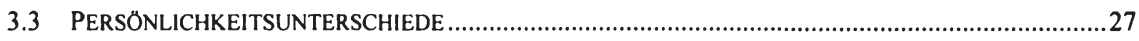

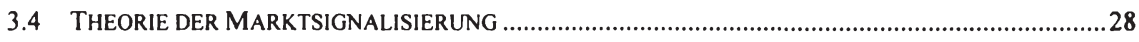

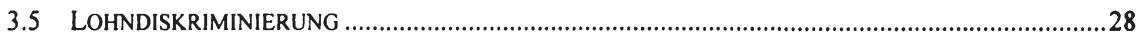

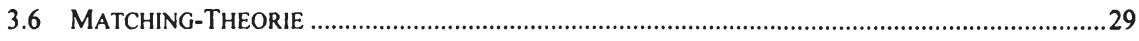

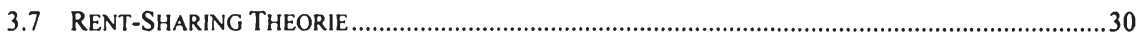

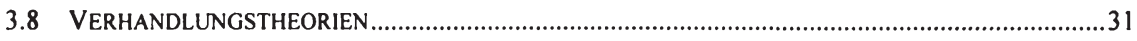

3.9 EFFIZIENZLOHNTHEORIE UND THEORIE DER RELATIVEN POSITION .............................................32



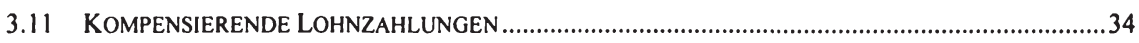

3.12 ZUSAMMENFASSUNG DER ALLGEMEINEN THEORIEN ZUR BESTIMMUNG DER LOHNHÖHE ........34

4 BESONDERHEITEN IN DER BESTIMMUNG DER LOHNHÖHE BEI NPO............................36

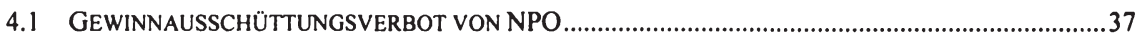

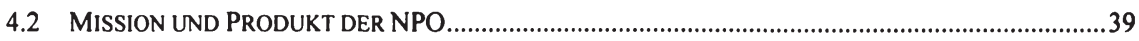

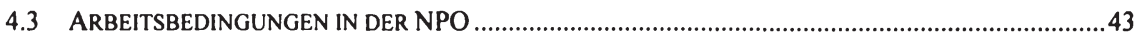

4.4 GEWERKSCHAFTLICHE ORGANISATION DER ARBEITNEHMER/-INNEN IN EINER NPO.................43

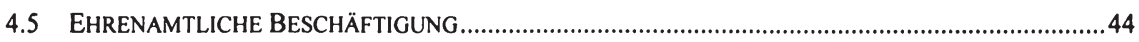

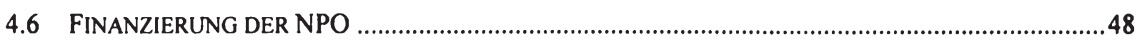

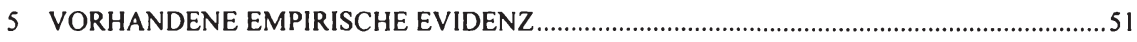




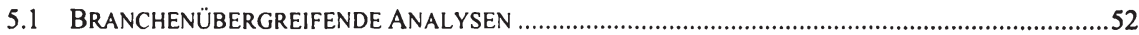

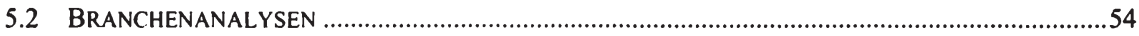

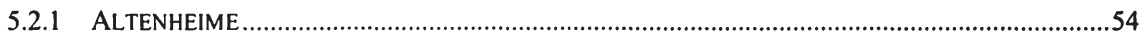

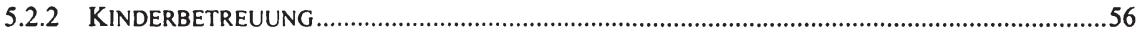

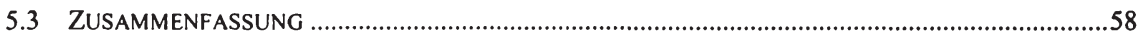

6 MODELLRAHMEN, VARIABLENAUSWAHL UND ERWARTETE VORZEICHEN .............61

6.1 DiE LOHNFUNKTION VON ARBEITNEHMER/-INNEN IM NONPROFIT SEKTOR .............................61

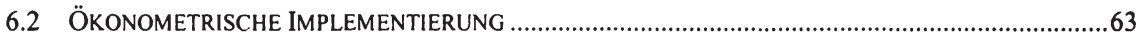

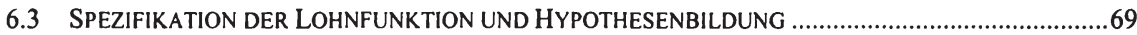

6.3.1 DER REGRESSAND: LOGARITHMIERTE BRUTTOTAGESLÖHNE ..............................................69

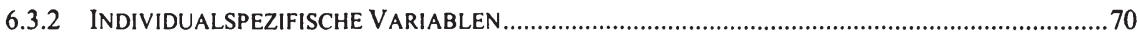

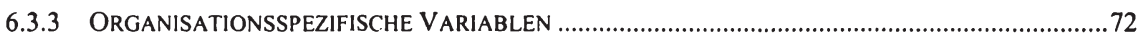

7 LINKED EMPLOYER-EMPLOYEE (LEE-) DATEN ALS GRUNDLAGE DER

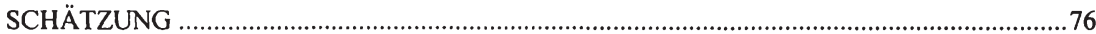

7.1 BESCHAFFENHEIT VON LEE-DATEN UND DEREN VORTEILE FÜR ARBEITSÖKONOMISCHE

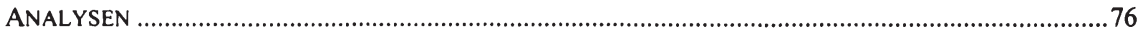

7.2 METHODISCHE ERFORDERNISSE BEI HIERARCHISCHEN DATENSTRUKTUREN.............................77

7.3 DER LEE-DATENSATZ „NPO-STEUER-DATEN 2006“..........................................................79

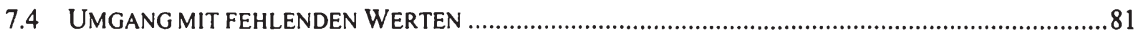

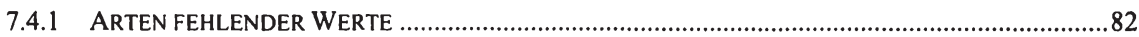

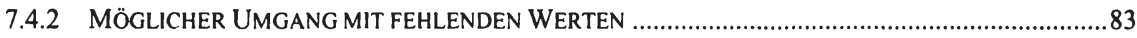

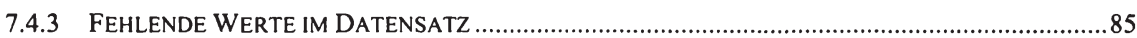

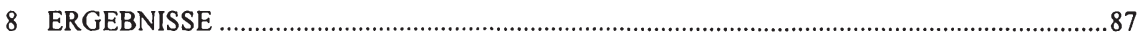

8.1 BESCHREIBUNG DER STICHPROBE UND DESKRIPTIVE ERGEBNISSE ..........................................87

8.2 KLEINSTE-QUADRAT-SCHÄTZUNG (OLS-REGRESSION) ........................................................92

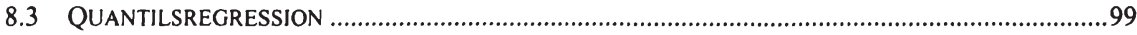

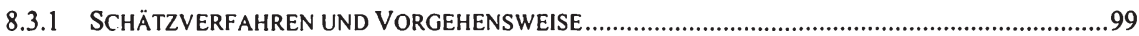

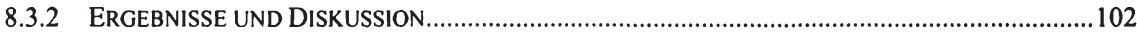

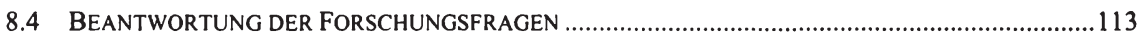

TEIL II: DETERMINANTEN DER INNERBETRIEBLICHEN LOHNSTREUUNG IM

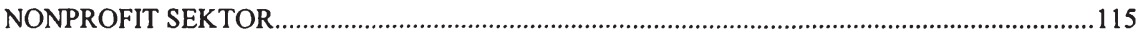

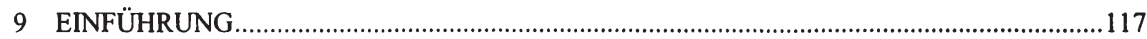

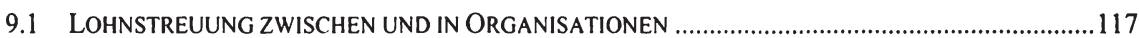


10 THEORIEN - WAS KANN DIE LOHNSTREUUNG IN ORGANISATIONEN BEEINFLUSSEN?

10.1 DIE ZUSAMMENSETZUNG DER ARBEITNEHMER/-INNEN IN ORGANISATIONEN:

HUMANKAPITALTHEORIE UND LOHNSTREUUNG .

10.2 KOLLEKTIVE LOHNVERHANDLUNGEN UND LOHNSTREUUNG - VERHANDLUNGSTHEORIEN ... 122

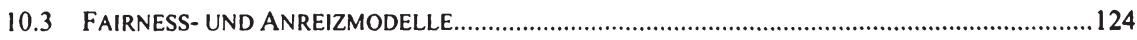

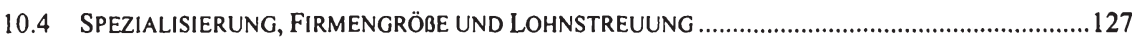

10.5 RENT-SHARING, EFFIZIENZLÖHNE UND KOMPENSIERENDE LOHNZAHLUNGEN .......................128

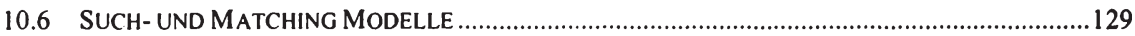

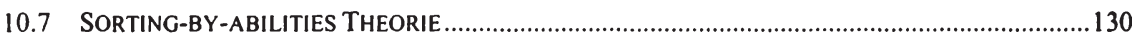

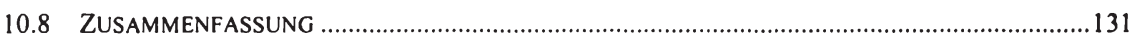

11 BESONDERHEITEN VON NPO IN BEZUG AUF DIE LOHNSTREUUNG ........................131

11.1 UNTERSCHIEDE BEZÜGLICH DER LOHNSTREUUNG ZWISCHEN DEM GEWINNORIENTIERTEN

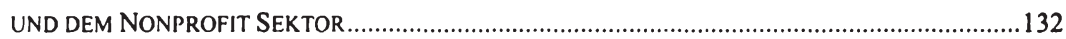

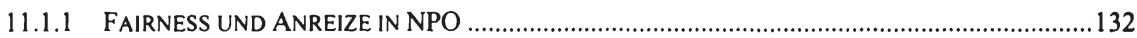

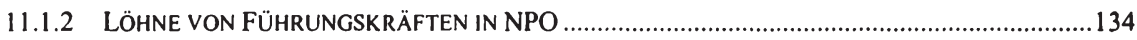

11.2 UNTERSCHIEDE BEZÜGLICH DER LOHNSTREUUNG ZWISCHEN NONPROFIT

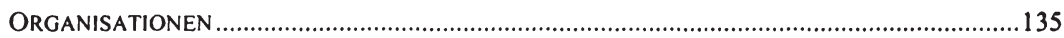



12.1 INNERBETRIEBLICHE LOHNSTREUUNG IM GEWINNORIENTIERTEN BEREICH.........................137

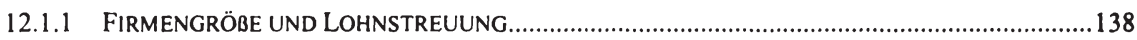

12.1.2 VERHANDLUNGSMACHT UND INNERBETRIEBLICHE LOHNSTREUUNG................................138

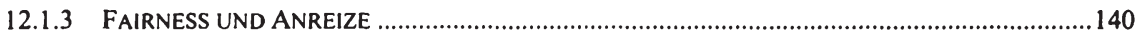

12.2 LOHNSPREIZUNG IN NPO UND NONPROFIT-NAHEN BRANCHEN .......................................... 142

12.3 LÖHNE VON FÜHRUNGSKRÄFTEN IM NONPROFIT SEKTOR ..................................................143

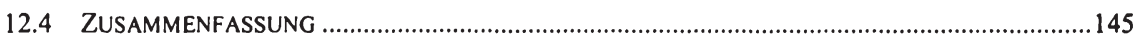

13 MODELLRAHMEN, VARIABLENAUSWAHL UND ERWARTETE VORZEICHEN .........150

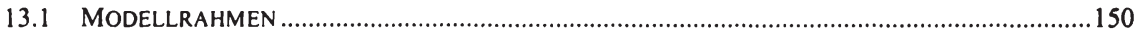

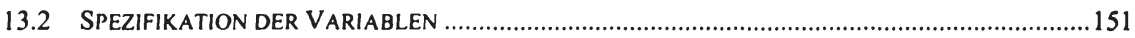

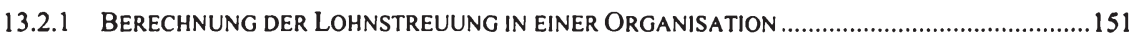

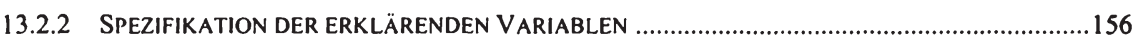

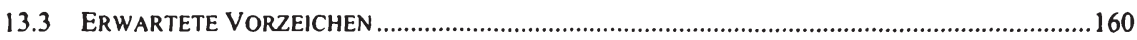


14.1 BESCHREIBUNG DES DATENSATZES UND DER KONTROLLVARIABLEN..................................... 164

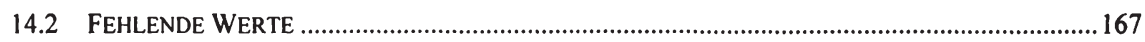

14.3 INSTRUMENTVARIABLENSCHÄTZUNG (IV SCHÄTZUNG ODER 2SLS-SCHÄTZUNG) ................. 168

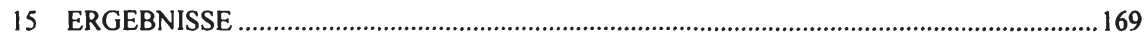

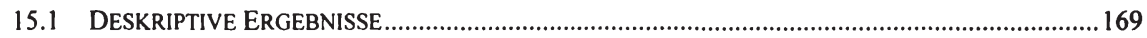

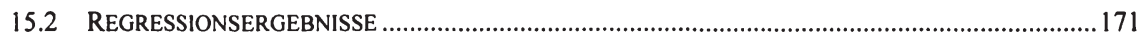

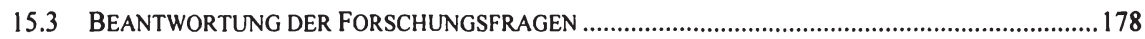

TEIL III: ZUSAMMENFASSENDE SCHLUSSBETRACHTUNG UND AUSBLICK ................... 181

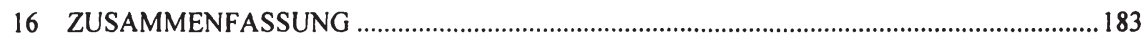

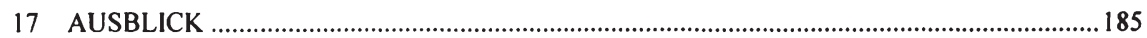

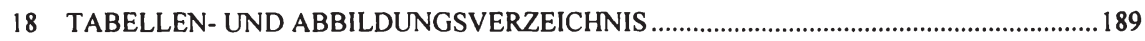

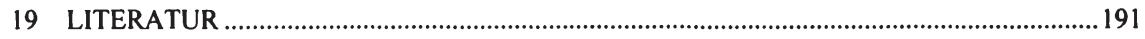


Diese beiden folgenden Stelleninserate wurden leicht verändert einer Zeitung entnommen wurden. Obwohl sie nur zwei Beispiele sind, sind sie doch typisch für Stellenanzeigen im Nonprofit Sektor:

NPO sucht für eine 30 Stunden Karenzvertretung eine/n Entwicklungspolitische/n Bildungs- und KampagnenreferentIn

Die NPO ist ein gemeinnütziger Verein, der im Bereich der Entwicklungszusammenarbeit weltweit Sozial-, Kinder- und Jugendprojekte unterstützt. In Österreich sind wir entwicklungspolitisch tätig: Wir gestalten Bildungsprogramme, sind Träger diverser Maßnahmen und Initiativen und machen Lobbying.

Sie arbeiten mit an der Konzeption, Durchführung und Dokumentation von entwicklungspolitischen Bildungs- und Kampagnenprojekten in Österreich. Projektformulierungen, Erstellung inhaltlicher Grundlagendokumente, Betreuung von Gruppen und Initiativen sowie Kontakt mit SpenderInnen bzw. Fördergruppen und Medien sind Teil Ihrer Arbeit. Als besonderer Schwerpunkt betreuen Sie im Jahr der Fußballeuropameisterschaft und Olympiade Projekte und Aktivitäten in Österreich zum Themenfeld Fußball/Sport und Entwicklung. Organisationstalent, genaues und selbständiges Arbeiten, Erfahrung im Bereich Entwicklungspolitik und Kampagnengestaltung, Kenntnis der für die Arbeit relevanten Förder- und Finanzierungsinstrumente sowie deren Handhabung sind erwünscht. Sehr gute Computerkenntnisse, ausgezeichnete Deutschkenntnisse, gute Stressbewältigung, Eigeninitiative, hohes Engagement und Freude an der Mitarbeit in einer Non Profit Organisation sind Voraussetzung.

Die Bezahlung erfolgt im NGO üblichen Gehaltsrahmen. Wir freuen uns über Ihre Bewerbung. 
CARE Österreich, Verein für Entwicklungszusammenarbeit und humanitäre Hilfe, sucht eine/n erfahrene/n Programm Officer (m/w) (Schwerpunkt Asien) 30 Stunden

CARE zählt zu den weltweit größten unabhängigen internationalen Hilfsorganisationen. 14.500 MitarbeiterInnen arbeiten in rund 70 Projektländern in Afrika, Asien, Lateinamerika und Osteuropa an der Vision einer Welt ohne Armut. CARE hat Beraterstatus I bei den Vereinten Nationen und ist politisch und weltanschaulich unabhängig.

\section{Aufgaben}

- Contract Management und Project Controlling

- Projektentwicklung für EU und ADA Ausschreibungen in den Regionen Asien \& CIS

- Liaison mit CARE Länderbüros, Donoren und Partnerorganisationen

- Projektreisen

\section{Anforderungsprofil}

- Abgeschlossenes Studium aus den Bereichen Sozialwissenschaften und/oder relevante Studien zur Entwicklungszusammenarbeit

- Mindestens 2 Jahre Erfahrung im Contract Management für EU und ADA finanzierte Projekte

- Sehr gute organisatorische Fähigkeiten

- Erfahrung in der Zusammenarbeit mit multi-nationalen und multi-kulturellen Teams

- Ausgezeichnete Deutsch- und Englischkenntnisse in Wort und Schrift

- Auslandserfahrung in einem EZA Projekt wünschenswert

\section{Was können wir Ihnen bieten?}

- 30 Stunden mit maximal $20 \%$ Reiseanteil

- Selbstständige, abwechslungsreiche Tätigkeit in einem dynamischen Umfeld

- Engagiertes Team

- NGO-adäquate Entlohnung

Quelle: derstandard.at (Abgerufen am 25.6.2008)

Die knappe Formulierung in den Stellenanzeigen wirft viele Fragen auf: Die Bezahlung, so im Text, erfolge im $\mathrm{NGO}^{2}$ (oder Nonprofit) üblichen Gehaltsrah-

1 http://derstandard.at/?url=/anzeiger/derjob/DetailView.aspx\%3FID\%3D108447

2 Non-Governmental Organisation 
men. Was hat sich der interessierte Leser bzw. die interessierte Leserin dieser Stellenanzeigen unter einer NPO-adäquaten Entlohnung vorzustellen? Ist damit ein vergleichsweiser niedriger Lohn gemeint? Sind Löhne im Nonprofit Sektor zwingenderweise niedriger als im gewinnorientierten Bereich? Ähneln sich alle Nonprofit Organisationen (NPO) in dieser Eigenschaft oder sind vielmehr konkrete Eigenschaften der Organisation ausschlaggebend in der Frage, wie hoch der Lohn sein wird? Verhält sich das Lohnschema innerhalb einer Nonprofit Organisationen für alle gleich?

Die vorliegende Arbeit hat das Ziel, einige dieser Fragen zu klären, indem sie sich mit Löhnen im österreichischen Nonprofit Sektor auseinandersetzt. Die Lohnhöhe und Lohnstreuung in Nonprofit Organisationen werden genauer analysiert. Dabei steht weniger der Vergleich von NPO mit gewinnorientierten Unternehmen im Mittelpunkt. Vielmehr soll hier die Frage beantwortet werden, ob besondere Eigenschaften der Nonprofit Organisationen die Lohnhöhe und die Lohnstreuung beeinflussen.

\subsection{Darstellung der Relevanz des Themas}

Das Einkommen aus unselbständiger Arbeit stellt für die meisten Personen den wichtigsten Bestandteil ihrer ökonomischen Lebensgrundlage dar. Löhne und Gehälter $^{3}$ sind am Arbeitsmarkt als ,Preis der Arbeit" von zentraler Bedeutung. Die Lohnstruktur ${ }^{4}$ in einer Gesellschaft ist sowohl von gesellschaftlicher als auch von ökonomischer Relevanz - einerseits unter Aspekten der Verteilungsgerechtigkeit, andererseits gibt es ökonomische Theorien, die einen Zusammenhang zwischen der Produktivität einer Firma und der Lohnstruktur herstellen (vgl. z.B. Winter-Ebmer, Zweimüller 1999). Aus diesen Gründen stellen die Löhne sowie die Lohnstruktur einen wichtigen Forschungsgegenstand in der Arbeitsmarktökonomie dar.

Nach dem neoklassischen Modell bestimmt sich die Höhe der Löhne auf einem vollkommenen Arbeitsmarkt durch das Gleichgewicht von Arbeitsangebot und nachfrage. Ein Unternehmen stellt Arbeitnehmer/-innen ein bis das Grenzerlösprodukt der Arbeit dem Lohn entspricht (vgl. z.B. Pindyck, Rubinfeld 2005: 679). Dieses einfache Modell kann der komplexen Lohnstruktur einer Volkswirtschaft nicht gerecht werden, die sich daraus ergibt, dass eine Vielzahl

3 Zur Definition der Begrifflichkeiten siehe Kapitel 1.2.1.

4 „Die Betrachtung der L[ohnstruktur] konzentriert sich auf die Lohndifferenzen, die durch interpersonelle Leistungs- und Qualifikationsunterschiede und durch die Unterschiedlichkeit der Berufe bedingt sind. Neben diesen "erlaubten" Lohndifferenzen sind aber auch interregionale, interbetriebliche und interindustrielle Unterschiede von Interesse" (Gabler Wirtschafts Lexikon 2004: 1932). 
von regionalen und qualifikationsspezifischen Teilarbeitsmärkten nebeneinander bestehen. Die Lohnfindung kann auf jedem dieser nicht immer „vollkommenen“ Märkte unabhängig voneinander zu einem unterschiedlichen Ergebnis kommen. Die Löhne auf unterschiedlichen Teilarbeitsmärkten können einander jedoch über die Zeit beeinflussen. Daher wurden verschiedene Theorien geschaffen, die die Determinanten des Lohns auf Branchenebene, sowie auf betrieblicher und individueller Ebene bestimmen. Besonders der Betrieb (bzw. synonym dazu die Firma oder Organisation) ist zuletzt in den Mittelpunkt des Interesses gerückt, da er die Lohnstruktur einer Gesellschaft maßgeblich beeinflusst (vgl. Stephan 2001: 13).

Im Jahr $2001^{5}$ gab es in Österreich 11.005 Arbeitsstätten, die dem Nonprofit Sektor zugehörig waren. Das entspricht 2,8\% aller österreichischen Arbeitsstätten. Insgesamt waren 2005 rund 170.000 entgeltlich Beschäftigte in Arbeitsstätten des Nonprofit Sektors tätig, wobei Werkvertragsnehmer/-innen in dieser Zählung nicht inkludiert sind (vgl. Haider et al. 2008: 757). Ein Großteil dieser Arbeitsplätze ist in der Dienstleistungsbranche zu finden. Nach einer Hochrechnung von Trukeschitz sind zum Zeitpunkt 31.12.2001 rund 55.200 Personen entgeltlich in den sozialen Diensten des Nonprofit Sektors beschäftigt (vgl. Trukeschitz 2006: 219).

Bis heute ist trotz der nicht zu vernachlässigenden Bedeutung des österreichischen Nonprofit Sektors nur wenig über Löhne in diesem Sektor bekannt. Das ökonomische Verhalten von NPO unterscheidet sich von gewinnorientierten Unternehmen in vielerlei Hinsicht (siehe dazu z.B. Hansmann 1980; James, Rose-Ackerman 1986), auch in Bezug auf das Lohnsetzungsverhalten (vgl. z.B. Leete 2001, Ruhm, Borkoski 2003, Preston 1988, Mocan, Tekin 2003). Löhne und Lohnstrukturen im Nonprofit Sektor sind wenig erforscht. Vorhandene Studien, die sich mit Löhnen im Nonprofit Sektor beschäftigen, untersuchen zumeist das Lohndifferenzial zwischen NPO und gewinnorientierten Unternehmen, bzw. ob ein derartiges Lohndifferenzial besteht. Die Ergebnisse der Studien sind zum Teil widersprüchlich (vgl. z.B. Leete 2001; Leete 2006 und Kapitel 5).

Vergleiche zwischen der Lohnhöhe in NPO und gewinnorientierten Unternehmen unterstellen häufig implizit, dass in beiden Bereichen vergleichbare Firmenlohnpolitiken gegenüber allen Arbeitnehmer/-innen der Organisation bestehen. Denkbar wäre es aber, dass bezüglich der innerbetrieblichen Lohnstreuung erhebliche Unterschiede zwischen NPO, gewinnorientierten und öffentlichen Unternehmen bestehen.

Vor diesem Hintergrund besteht das Ziel der Dissertation darin, die Lohnhöhe von bezahlten Arbeitnehmer/-innen im Nonprofit Sektor sowie die inner-

5 Neuere Daten sind meines Wissens nach nicht verfügbar. 
betriebliche Lohnstreuung in österreichischen NPO genauer zu analysieren. Im Mittelpunkt des Interesses steht dabei die Frage, ob Zuwendungen an eine Organisation, die Lohnhöhe beeinflussen. Freiwillige Mitarbeiter/-innen, Spenden und Subventionen sind für den Nonprofit Sektor charakteristisch, wie in den kommenden Kapiteln gezeigt werden wird. Ehrenamtliche Mitarbeit stellt eine zeitliche Ressource dar, Spenden und Subventionen sind monetäre $\mathrm{Zu}$ wendungen, die das Budget der Organisation erweitern.

\subsection{Begriffsbestimmung}

Vor der Präsentation der Forschungsfragen der Arbeit werden einleitend zentrale Begriffe der Arbeit definiert und abgegrenzt. Dazu zählen die Termini ,Lohn' und ,Nonprofit Organisation'.

\subsubsection{Lohn}

Im engeren Sinn ist der Lohn das „Arbeitseinkommen gewerblicher Arbeitnehmer[/-innen] (Arbeiter[/-innen])" (Meyers Großes Taschenlexikon in 24 Bänden : o.S.) oder das „Entgelt für diejenige Arbeit, die in Abhängigkeit von einem Arbeitgeber geleistet wird" (Geigant et al. 2000: 599). Im weiteren Sinn ist der Lohn das „Entgelt für menschliche Arbeitsleistung“ (Geigant et al. 2000: 599) bzw. das „Arbeitseinkommen der Arbeitnehmer[/-innen]“, was auch das Arbeitsentgelt von Angestellten, sowie Beamten und Beamtinnen beinhaltet (Lexikon der Geographie 2001: o.S.). Der Begriff ,Löhne und Gehälter' ist die ,zusammenfassende Bezeichnung für die Bruttobeträge der Arbeitsentgelte. $\mathrm{Zu}$ Löhnen und Gehältern gehören alle Vergütungen, die die Belegschaftsmitglieder (Arbeiter[/-innen] und Angestellte) und Geschäftsführer[/-innen] sowie Mitglieder des Vorstands erhalten, gleichgültig in welcher Form sie gewährt werden, also auch Sachbezüge, Aufwandsentschädigungen etc." (Gabler Wirtschafts Lexikon 2004: 1921). Synonyme zum Begriff sind auch ,Entgelt', ,Entlohnung', , Vergütung' und ,Verdienst'.

Während das Gehalt das Arbeitsentgelt für Angestellte darstellt, ist der Lohn jenes für Arbeiter/-innen. Wie in der Ökonomie üblich, werden in dieser Arbeit Löhne und Gehälter zusammenfassend als ,Löhne' bezeichnet, wobei hier jene Zahlungsströme gemeint sind, die an den Produktionsfaktor Arbeit gezahlt werden. Im empirischen Teil dieser Arbeit werden nur Bruttolöhne ohne Sachbezüge und Aufwandsentschädigungen aufgrund der Datenverfügbarkeit als Maßgröße herangezogen. 


\subsubsection{Nonprofit Organisationen und der Nonprofit Sektor}

Der Begriff ,Nonprofit Organisation' $\left(\mathrm{NPO}^{6}\right)$ hat viele Synonyme. Im Englischen sind neben dem Begriff der Nonprofit Organisation auch ,not-for-profit organisation', ,nonprofit enterprise' (James, Rose-Ackerman 1986), ,non-profitdistributing organisation', ,philantropic organisation', ,voluntary organisation', ,social-profit organisation' und ,charitable organisation' üblich. Im deutschen Sprachgebrauch sind unter anderem auch ,Freiwilligenorganisation' und ,zivilgesellschaftliche Organisation' üblich.

Das Wort Nonprofit Organisation ist eine Negativ-Abgrenzung gegenüber den gewinnorientierten Firmen, was mitunter als problematisch gesehen wird, da sich NPO dadurch ausschließlich durch eine Abgrenzung zu den anderen Organisationen charakterisieren und identifizieren. ${ }^{7}$, Nonprofit' heißt nicht, wie aus dem Namen geschlossen werden könnte, dass eine NPO keine Gewinne machen darf. Der Name soll vielmehr ausdrücken, dass eine NPO nicht gewinnorientiert ist, weshalb der Begriff ,not-for-profit organisation' eigentlich treffender wäre. Entscheidend ist, dass NPO anders als Firmen, nicht gewinnmaximierend arbeiten, sondern andere Ziele wie zum Beispiel die Erreichung gewisser Sachziele im Vordergrund haben. ${ }^{8}$

Die Begriffe ,Nongovernmental Organisation' (NGO) bzw. ,Nichtregierungsorganisation' (NRO) werden auch als Synonym von NPO betrachtet. Sie drücken aus, dass die Organisation nicht staatlich ist. Dementsprechend müssten sowohl gewinnorientierte als auch nicht gewinnorientierte (d.h. Nonprofit) Organisationen in diesem Begriff enthalten sein. Üblicherweise werden aber nur NPO darunter verstanden.

In dieser Arbeit wird das Wort Nonprofit Organisation (NPO) verwendet, da es in der Literatur sehr weit verbreitet ist.

Nach dem Johns Hopkins Comparative Non-Profit Sector Project werden NPO durch fünf Kriterien definiert. NPO

- müssen private, d.h. nicht staatliche Organisationen sein;

- müssen ein Mindestmaß an Selbstverwaltung bzw. Entscheidungsautonomie aufweisen;

6 In dieser Arbeit steht die Abkürzung NPO sowohl für „Nonprofit Organisation“ als auch für die Mehrzahl „Nonprofit Organisationen“.

7 Zur Problematik der Begriffsstreitigkeit von NPO siehe auch: Simsa, Meyer (2006)

8 Zu einer Diskussion über mögliche Zielsetzungen einer NPO siehe z.B. James, RoseAckerman (1986), Steinberg (2006) und Brown, Slivinski (2006). 
- dürfen keine Gewinne an Eigentümer/-innen oder Mitglieder ausschütten;

- besitzen ein Mindestmaß an formaler Organisation und

- zeichnen sich durch ein Minimum an Freiwilligkeit aus (Badelt 2002: 8 f.). ${ }^{9}$

Dieser sehr gängigen Definition einer NPO wird in dieser Arbeit gefolgt.

Der Nonprofit Sektor umfasst die Gesamtheit aller NPO in einer Volkswirtschaft. Im deutschen Sprachraum sind auch die Begriffe ,Sozialwirtschaft', ,Freiwilligensektor', ,Zivilgesellschaft' und ,Dritter Sektor' Umschreibungen des Nonprofit Sektors. Der Nonprofit Sektor gilt dabei neben dem Staat und dem privaten gewinnorientierten Sektor als ,Dritter Sektor'.

NPO werden in der volkswirtschaftlichen Gesamtrechnung anhand ihrer Finanzressourcen definiert. Eine NPO im System of National Accounts wird dann als ,private Organisationen ohne Erwerbszweck“ anerkannt, wenn mehr als 50\% ihrer Einnahmen nicht vom Verkauf von Waren oder Dienstleistungen stammen. Einnahmen müssen mehrheitlich aus Spenden oder Mitgliedsbeiträgen von privaten Haushalten stammen. Außerdem dürfen sie nicht vom Staat kontrolliert werden, ansonsten werden die Organisationen dem Sektor Staat zugewiesen. Organisationen von geringer Bedeutung werden dem Sektor ,private Haushalte“ zugerechnet (vgl. Haider et al. 2008; Heitzmann 2001: 67). Die Defintion nach dem System of National Accounts kann zur Folge haben, dass eine Organisation, deren Finanzierungsstruktur jährlichen Schwankungen unterliegt, in einem Jahr als private Organisation ohne Erwerbszweck definiert wird, im darauffolgenden Jahr z.B. als Marktproduzentin angesehen werden könnte, wenn in diesem Jahr mehr als 50\% der Einnahmen von Umsatzerlösen stammen.

Die Tätigkeiten von NPO haben meist gemeinnützigen Charakter. Ordnet man NPO nach Branchen, so sind sie hauptsächlich in den Aktivitätsbereichen „Kultur, Sport, Freizeit“, „Bildung und Forschung“, „Gesundheit“, „Soziale Dienste“, „Umwelt“, „Lokale Entwicklung und Wohnungswesen“, „Rechtswesen, Interessensvertretung, Politik“, „Stiftungs- und Spendenwesen, Freiwilligenarbeit“, „Internationale Aktivitäten“, „Religion“ und „Wirtschafts- und Berufsverbände, Gewerkschaften" tätig (vgl. UN 2003: 26ff. und Salamon, Anheier 1996: 7).

NPO spielen eine wichtige Rolle in einer Gesellschaft und Volkswirtschaft. Sie sind wichtiger Bestandteil des kulturellen, sozialen und politischen Systems. Auch ihr volkswirtschaftlicher Beitrag in einer Gesellschaft ist nicht zu vernachlässigen. Für einen Überblick über die Strukturen und Ausmaß des österrei-

9 Für eine ausführliche Diskussion des NPO-Begriffs siehe z.B.: Ostermeier (2002). 
chischen NPS siehe auch Haider et al. (2008), Heitzmann (2001), Neumayr et al. (2007), Schneider (2007) und (2008) .

\subsection{Forschungsfragen der Dissertation}

Eine quantitative Analyse der Lohnstruktur im österreichischen Nonprofit Sektor wurde bislang noch nicht durchgeführt. Ungeklärt bleiben daher die Fragen, wie Löhne im Nonprofit Sektor und innerhalb einer Nonprofit Organisationen differieren und ob das Lohnsetzungsverhalten einer Organisation für die ganze Belegschaft konsistent ist oder ob der Arbeitsmarkt innerhalb einer Organisation segmentiert ist.

Ziel dieser Arbeit ist es, verschiedene Aspekte der Lohnstruktur von Nonprofit Organisationen näher zu beleuchten. Dabei liegt ein besonderer Schwerpunkt bei jenen Determinanten, die das Budget, d.h. die Zahlungsfähigkeit der Organisation womöglich erweitern und typisch für den Nonprofit Sektor sind. Dazu zählen Spenden von Zeit und Geld sowie öffentliche Subventionen.

Forschungsfragen, die durch die Dissertation beantwortet werden sollen, sind insbesondere:

Welche Determinanten wirken auf die Lohnhöhe und die innerbetriebliche Lohnstreuung in NPO in Österreich?

Und spezieller:

Beeinflussen ehrenamtliche Mitarbeiter/-innen, Geldspenden und öffentliche Subventionen die Lohnhöhe und die innerbetriebliche Lohnstreuung von Nonprofit Organisationen?

\subsection{Aufbau der Arbeit}

Die Arbeit ist in drei Hauptteile gegliedert. Im ersten Teil (Kapitel 2 bis 8) werden Determinanten der Lohnhöhe von Arbeitnehmer/-innen im Nonprofit Sektor analysiert, im zweiten (Kapitel 9 bis 15) werden Determinanten der innerbetrieblichen Lohnstreuung in NPO untersucht. Der dritte Teil (Kapitel 16 und 17) fasst die wichtigsten Ergebnisse beider Teile zusammen und gibt einen Ausblick.

Beide Teile können auch getrennt gelesen werden, da sie jeweils in sich geschlossen sind. Der erste Teil, der sich mit der Lohnhöhe von bezahlten Arbeitnehmer/-innen im Nonprofit Sektor beschäftigt, beginnt mit einem einleitenden Kapitel, danach werden in den folgenden zwei Kapiteln die wichtigsten vorhandenen Theorien zum Thema zusammengefasst. Das nächste Kapitel gibt einen Überblick über wichtige, vorhandene empirische Arbeiten Kapitel 6 stellt das 
geschätzte Modell dar, danach wird die Berechnungsmethode gezeigt. Die Ergebnisse werden im Kapitel 8 dargestellt. Analog dazu verläuft der zweite Teil der Dissertation. Auch hier werden nach einem einleitenden Kapitel, wichtige Theorien, die Aussagen über innerbetriebliche Lohnstreuung treffen und danach empirische Arbeiten vorgestellt. Kapitel 13 präsentiert den Modellrahmen für die quantitative Schätzung, die Ergebnisse des zweiten Teils werden im Kapitel 15 dargestellt. 
Astrid Haider - 978-3-631-75377-4

Downloaded from PubFactory at 01/11/2019 05:43:53AM

via free access 


\section{Teil I:}

Determinanten der Lohnhöhe von bezahlten Arbeitnehmer/-innen in Nonprofit Organisationen 
Astrid Haider - 978-3-631-75377-4

Downloaded from PubFactory at 01/11/2019 05:43:53AM

via free access 
Löhne sind ein komplexes und wichtiges Thema für alle Organisationen, in denen Individuen bezahlt beschäftigt sind, da Löhne sowohl das Wohlbefinden der Arbeitnehmer/-innen als auch den Erfolg der Organisation beeinflussen. Sie wirken sich unter anderem auf die Motivation, Arbeitszufriedenheit, Produktivität, Arbeitnehmerfluktuation, auf Kosten und Erlöse aus, wobei nicht nur die individuelle Lohnhöhe ausschlaggebend ist, sondern auch die Lohnstreuung oder die Änderung der Löhne im Zeitverlauf.

Der erste Teil der Dissertation setzt sich mit der Lohnhöhe von bezahlten Arbeitnehmer/-innen in österreichischen NPO auseinander. Es stellt sich die Frage, ob Eigenheiten von NPO, die charakteristisch für den dritten Sektor sind, die Lohnhöhe in NPO beeinflussen, womit z.B. auch Lohnunterschiede zwischen dem Nonprofit und gewinnorientierten Sektor erklärt werden könnten.

Zur Lohnhöhe im Nonprofit Sektor existieren einige empirische Analysen, die zumeist die Lohnhöhe zwischen dem Nonprofit und Forprofit, d.h. dem gewinnorientierten Sektor vergleichen, entweder branchenübergreifend oder in bestimmten Branchen wie der Kinderbetreuung oder der Altenpflege. Es zeigt sich, dass die Ergebnisse dieser vergleichenden Studien stark vom verwendeten $\mathrm{Da}$ tensatz und den in die Lohnfunktion aufgenommenen Kontrollvariablen abhängen. Zumeist wurden entweder Individual- und/oder Organisationscharakteristika als Kontrollvariable verwendet, die die Lohnhöhe eines Individuums beeinflussen. Studien, die für die Analyse der Lohnhöhe in NPO Individualdaten verwenden sind z.B. Goddeeris (1988), Leete (2001), Preston (1989 und 1990), Shackett und Trapani (1987), Ruhm und Borkoski (2003) und Weisbrod (1983), Organisationsdaten verwendet beispielsweise Preston (1988). In der nun vorliegenden Dissertation werden beide Datenarten verbunden, um eine umfassendere Analyse vornehmen zu können. Bislang existieren nur wenige Studien, die auf derartigen LEE-Daten ${ }^{10}$, das sind Daten die aus Arbeitgeber- und Arbeitnehmerinformationen bestehen, basieren (vgl. Borjas et al. 1983; Holtmann, Idson 1993; Mocan, Tekin 2003; Noguchi, Shimizutani 2007).

Nach dem John Hopkins Comparative Non-profit Sector Project werden NPO, wie in Kapitel 1 verdeutlicht, durch fünf Charakteristika definiert: NPO besitzen nach dieser Definition (i) ein Mindestma $\beta$ an formaler Organisation, (ii) sind privat, d.h. nicht staatlich, (iii) weisen ein Minimum an Selbstverwaltung bzw. Entscheidungsautonomie auf, (iv) dürfen keine Gewinne an Eigentümer/-innen oder Mitglieder ausschütten und ( $v$ ) besitzen ein Mindestmaß an Freiwilligkeit (vgl. Badelt et al. 2007b: 7). Besonders durch die letzten zwei Punkte unterscheiden sich NPO von gewinnorientierten Unternehmen. In dieser Arbeit wird

10 LEE steht für linked employer-employee. Auch üblich ist die Verwendung des Begriffs matched employer-employee. 
argumentiert, dass diese Eigenheiten der NPO bei der Analyse von Löhnen berücksichtigt und Lohnfunktionen dementsprechend adaptiert werden müssen.

Interessanterweise wird in der vorhandenen Literatur zwar betont, dass das Gewinnausschüttungsverbot, dem NPO unterliegen, eine wichtige Eigenschaft ist, die in die Lohnanalyse miteinbezogen werden muss, das Vorhandensein von Freiwilligkeit in der Organisation spielt hingegen in existierenden Studien weitgehend keine Rolle bei der Modellierung von Löhnen im Nonprofit Sektor.

Der Fokus dieser Arbeit liegt daher auf dem Einfluss dieser freiwilligen Elemente einer NPO auf die Lohnhöhe von bezahlten Arbeitnehmer/-innen in NPO, insbesondere von Beiträgen in Form von Zeit (d.h. freiwillige, unbezahlte Arbeit) und Geld (d.h. Spenden). Am Rande werden auch Subventionen dazu gezählt. Insgesamt erhöhen diese Zuwendungen die zeitlichen und monetären Ressourcen einer Organisation, d.h. die Budgetbeschränkung einer Organisation wird dadurch gelockert. Über Zuwendungen in Form von freiwilliger Arbeitsleistung und Geldspenden können NPO relativ frei verfügen, das Budget der Organisation wird dadurch erweitert. Unklar ist jedoch, inwieweit NPO auch über Subventionen frei verfügen können.

Doch auch in der freien Verfügbarkeit über ehrenamtliche Arbeit und Spenden liegen Grenzen. Zum einen können durch gesetzliche Vorschriften und Auflagen freiwillige Mitarbeiter/-innen nicht in allen Tätigkeitsfeldern eingesetzt werden, zum anderen sind verschiedene Tätigkeiten für Ehrenamtliche unterschiedlich attraktiv. Spenden werden teilweise für bestimmte Projekte gegeben, sodass auch dann die NPO nicht völlig frei über die Gelder handhaben kann.

Die Forschungsfragen des ersten Teils sind demnach:

Beeinflussen Ehrenamtlichkeit, Spenden und Subventionen (inkl. Einnahmen aus Leistungsverträgen) die Lohnhöhe von bezahlten Arbeitnehmer/-innen in NPO?

Wenn diese Elemente eine Rolle bei der Bestimmung der Lohnhöhe spielen, auf welche Art und Weise beeinflussen sie die Lohnhöhe?

Die Forschungsfragen werden mithilfe einer OLS-Regression und einer Quantilsregression beantwortet. Letztere ermöglicht eine genauere Analyse möglicher Zusammenhänge, da der Einfluss der unabhängigen Variablen auf verschiedene Quantile der abhängigen Variablen separat geschätzt werden kann. Die Quantilsregression wurde für die Analyse der Lohnhöhe in NPO meines Wissens nach noch nicht verwendet, obwohl diese Methode zur Schätzung der Lohnfunktion in anderen Bereichen durchaus üblich ist (vgl. z.B. Blaise 2005; Machado, Mata 2005).

In Folge werden im Kapitel 3 allgemeine Theorien zur Bestimmung der Lohnhöhe dargestellt und danach im Kapitel 4 auf Eigenheiten von NPO, die bei der 
Schätzung der Lohnhöhe zu beachten sind, eingegangen. Kapitel 5 stellt vorhandene Analysen zur Lohnhöhe in NPO kurz dar, bevor im Kapitel 6 ein Modell zur Bestimmung der Lohnhöhe im Nonprofit Sektor aufgestellt wird. Daraus wird eine empirisch schätzbare Lohnfunktion abgeleitet. Kapitel 7 stellt die Datengrundlage, auf der die empirische Schätzung beruht vor, außerdem werden methodische Anmerkungen zur Berechnung der Ergebnisse getroffen. Kapitel 8 stellt schließlich die Ergebnisse der empirischen Analyse dar und beantwortet die Forschungsfragen des ersten Teils der Arbeit.

\section{Allgemeine Theorien zur Bestimmung der Lohnhöhe}

Einführend wird ein Überblick über gängige Theorien zur Bestimmung der Lohnfunktion gegeben. Auf eine ausführliche Darstellung wird jedoch verzichtet, da diese Theorien in Lehrbüchern zur Arbeitsökonomie nachzulesen sind (vgl. z.B. Franz 2006). Ebenso wird darauf verzichtet, für den Nonprofit Sektor wenig relevante Erklärungsansätze darzustellen.

\subsection{Neoklassisches Modell}

Nach dem neoklassischen Modell bestimmt sich die Höhe der Löhne auf einem vollkommenen Arbeitsmarkt durch das Gleichgewicht von Arbeitsangebot und nachfrage, wobei im sehr einfachen Modell davon ausgegangen wird, dass alle Arbeitnehmer/-innen Arbeit gleicher Qualität anbieten. Angebots- und Nachfragekurven werden aus dem mikroökonomischen Kalkül der jeweiligen Akteure abgeleitet (vgl. Klodt 2000: 18).

Ein Individuum entscheidet aus seiner individuellen Nutzenfunktion, wieviel Arbeit es zu einem bestimmten Lohnsatz anbieten möchte. Arbeitnehmer/-innen bieten solange Arbeit an, bis der Marktlohn ihrem Reservationslohn entspricht. Über alle Haushalte kann daraus die aggregierte Arbeitsangebotsfunktion abgeleitet werden.

Ein Unternehmen stellt Arbeitnehmer/-innen ein, bis das Grenzerlösprodukt der Arbeit dem Lohn entspricht. Das Grenzerlösprodukt der Arbeit stellt den Grenzerlös einer Produktionsmenge dar, die aus der zusätzlich produzierten Menge eines zusätzlich eingestellten Arbeiters bzw. einer Arbeiterin entsteht. Bei Unterstellung eines sinkenden Grenzprodukts der Arbeit hat die Arbeitsnachfragefunktion einen fallenden Verlauf.

Der Arbeitsmarkt wird als Wettbewerbsmarkt gesehen, auf dem Arbeiter/-innen gemäß ihrem Grenzprodukt entlohnt sind. Der Lohnsatz ergibt sich auf dem Wettbewerbsmarkt und verändert sich durch eine Veränderung von Angebot und Nachfrage. Eine zentrale Voraussetzung ist ein frei schwankender Lohnsatz, der 
im Gleichgewichtssatz für die Räumung der Märkte sorgt (vgl. z.B. Pindyck, Rubinfeld 2005: 679 und 828).

Dieses einfache Modell kann der komplexen Lohnstruktur einer Volkswirtschaft nicht gerecht werden, die sich daraus ergibt, dass eine Vielzahl von regionalen und qualifikationsspezifischen Teilarbeitsmärkten nebeneinander bestehen. Die Lohnfindung kann auf jedem dieser Märkte unabhängig voneinander zu einem unterschiedlichen Ergebnis kommen. Die Löhne auf verschiedenen Teilarbeitsmärkten können einander jedoch über die Zeit beeinflussen. Daher wurden verschiedene Theorien geschaffen, die die Determinanten des Lohns auf Branchenebene, sowie auf betrieblicher und individueller Ebene bestimmen. Besonders der Betrieb (bzw. synonym dazu die Firma oder Organisation) ist zuletzt in den Mittelpunkt des Interesses gerückt, da er die Lohnstruktur einer Gesellschaft maßgeblich beeinflusst (vgl. Stephan 2001: 13).

$\mathrm{Zu}$ unterscheiden ist zwischen Theorien zur Bestimmung der individuellen Lohnhöhe die aus Unterschieden zwischen Individuen resultieren und jenen, die Differenzen durch Organisationsspezifika erklären. In einem vollkommenen Arbeitsmarkt werden Arbeitnehmer/-innen nach ihrer Produktivität bezahlt, d.h. dass Arbeitnehmer/-innen mit gleicher Produktivität in unterschiedlichen Organisationen denselben Lohn bekommen und Arbeitnehmer/-innen ungleicher Produktivität in derselben Firma ungleichen Lohn. Der Lohn wird ausschließlich durch die Produktivität der Person bestimmt, nicht durch firmenrelevante Faktoren. Lohnfunktionen spiegeln diese Tatsache wieder, indem die darin enthaltenen erklärenden Variablen hauptsächlich persönliche Eigenschaften der Personen sind (vgl. Manning 1994: 1). Das Modell wird der Realität jedoch oft nicht gerecht. Individuen gleicher Produktivität bekommen in verschiedenen Firmen unterschiedlichen Lohn, umgekehrt bekommen oft Individuen ungleicher Produktivität denselben Lohn in einer Firma, obwohl der Arbeitgeber die Unterschiede in der Produktivität beobachten kann. Löhne hängen häufig von Firmen oder bestimmten Arbeitsplätzen ab und weniger von Individuen. Die Tatsache betitelt Manning (vgl. 1994: 1) company wage policy bzw. Firmenlohnpolitik.

Die Humankapitaltheorie, die Theorien zu unbeobachteten Qualitätsunterschieden, zu Lohndiskriminierung, die Theorie zur Marktsignalisierung sowie die Matching-Theorie vermuten Lohnunterschiede aufgrund von (vermeintlichen) Produktivitätsunterschieden zwischen Individuen, alle weiteren dargestellten Theorien erklären Lohnunterschiede durch Unterschiede auf Organisationsebene.

\subsection{Humankapitaltheorie}

Die Humankapitaltheorie stellt ausgehend von Mincer (1958) und Schultz (1963) eine der bekanntesten Ansätze zur Erklärung der Lohnhöhe eines Arbeitnehmers bzw. einer Arbeitnehmerin dar. Dabei wird das Arbeitsangebot in den Mittelpunkt des Interesses gerückt. Die konventionelle Markttheorie wird dabei 
erweitert, indem die Heterogenität der Faktors Arbeit analytisch erfasst wird. Der Theorie liegt der Gedanke zugrunde, dass unterschiedlich hohe Investitionen in Humankapital beobachtbare Einkommensunterschiede zwischen Arbeitnehmer/-inne/n erklären. Humankapital stellt die Summe aus Wissen und Fertigkeiten eines Arbeitnehmers bzw. einer Arbeitnehmerin dar. Investitionen in das Humankapital können sowohl Zeit als auch Sachgüter sein, die für den Erwerb einer produktivitätserhöhenden Ausbildung aufgewendet werden. Über die Zeit wird Humankapital abgeschrieben, d.h. es verliert an Wert, weil erlerntes Wissen wieder vergessen oder weil es aufgrund technischer Erneuerungen obsolet wird. Humankapital wird üblicherweise in allgemeines und spezifisches Humankapital unterteilt, wobei ersteres nicht an Wert verliert, wenn der Arbeitgeber gewechselt wird, letzteres bei einem Arbeitsplatzwechsel wertlos wird.

Die Humankapitaltheorie kann erklären, warum Arbeitnehmer/-innen, die über eine längere Ausbildung verfügen, mehr verdienen als Arbeitnehmer/-innen, die wenig in Humankapital investiert haben. Dabei wird unterstellt, dass mehr Humankapital zu Produktivitätssteigerungen führt, die mit höheren Einkommenszahlungen einhergehen. Eine zusätzliche Investition in das Humankapital ist dann lohnend, wenn die abgezinsten Kosten des Erwerbs einer weiteren Einheit an Humankapital niedriger oder gleich den abgezinsten zukünftigen Rückflüssen aus dieser zusätzlichen Investition sind. Die Kosten der Investition bestehen aus Ausbildungskosten und Opportunitätskosten, die Erträge daraus sind Einkommenszahlungen, die über das Einkommen des Arbeitnehmers bzw. der Arbeitnehmerin, das er oder sie ohne weitere Ausbildungsaktivitäten erzielen könnte, hinausgehen.

Empirisch wurde versucht, eine quantitative Schätzung von Bildungsrenditen vorzunehmen, wobei eine exakte Messung schwierig ist. Die Erfassung der Berufserfahrung ist dabei weitaus schwieriger als die Erfassung der schulischen Ausbildung. Quantitative Analysen kommen zu dem Schluss, dass die Humankapitaltheorie etwa ein Drittel der beobachtbaren Einkommensvariation erklären kann (vgl. Franz 2006: 75-100).

\subsection{Persönlichkeitsunterschiede}

Arbeitnehmer/-innen können auch unterschiedlich entlohnt werden, da sie sich in ihren unveränderlichen Fähigkeiten und Persönlichkeitstypen unterscheiden (vgl. z.B. Nyhus, Pons 2006). ${ }^{11}$ Individuen unterscheiden sich nicht aufgrund

$11 \mathrm{Zu}$ unterschiedlichen Persönlichkeitstypen von Arbeitnehmer/-innen und den Auswirkungen auf die Arbeitsleistung bzw die Lohnhöhe siehe z.B. die vorwiegend psychologische Literatur: Burke, Witt (2004), John, Srivastava (1999), Keller (2007), Mueller, Plug (2006), Nyhus, Pons (2006), Sturman (1999) 
erworbener Fähigkeiten, sondern aufgrund unterschiedlicher Persönlichkeitstypen. Beispiele hierfür sind Unterschiede im Talent, der Motivation eines Arbeitnehmers bzw. einer Arbeitnehmerin, der Präferenz für Herausforderungen in der Arbeit und der Freude an Arbeitsaufträgen (vgl. Sturman 1999: 190). So wirkt sich zum Beispiel die emotionale Stabilität eines Individuums positiv auf die Lohnhöhe aus, Gewissenhaftigkeit positiv vor allem am Beginn der Arbeitskarriere eines Individuums (vgl. Nyhus, Pons 2006). Diese Persönlichkeitsunterschiede der Arbeitnehmer/-innen führen zu Produktivitätsunterschieden, was wiederum zu Unterschieden in der Entlohnung führt. In empirischen Untersuchungen werden verschiedenartige Persönlichkeitstypen nur selten analysiert, da sie zum größten Teil unbeobachtbar sind oder in vorhandenen Datensätzen nicht beobachtet wurden (vgl. Becker 2000: 95ff.).

\subsection{Theorie der Marktsignalisierung}

Im Unterschied zur Humankapitaltheorie wird bei der Theorie der Marktsignalisierung davon ausgegangen, dass Ausbildung nicht notwendigerweise produktivitätssteigernd ist, sich aber dennoch für Arbeitnehmer/-innen lohnen kann. Der Theorie liegt eine Informationsasymmetrie zwischen Arbeitgeber und Arbeitnehmer/-in zugrunde. Der oder die Einzelne weiß über die eigene Produktivität Bescheid, der Arbeitgeber kann dies beim Einstellungsgespräch oder unmittelbar nach der Einstellung jedoch nicht feststellen, bzw. wäre dies mit sehr hohen Kosten verbunden. Deshalb geht der Arbeitgeber von einer durchschnittlichen Produktivität der Arbeitnehmer/-innen aus. Personen mit hoher Produktivität sind infolge unterbezahlt und müssen daher höhere Produktivität signalisieren. Eine Möglichkeit, dem Arbeitgeber gegenüber höhere Produktivität zu signalisieren, sind Bildungsabschlüsse. Ausbildung ist somit ein Signal für hohe Produktivität der Arbeitnehmer/-innen, an das sie höhere Lohnforderungen knüpfen (vgl. z.B. Pindyck, Rubinfeld 2005: 808f.). Deshalb ist der Theorie der Marktsignalisierung zufolge höhere Ausbildung mit höheren Lohnzahlungen verbunden, Ausbildung ist jedoch, wie gezeigt, nicht notwendigerweise der Grund für die höhere Produktivität der besser Bezahlten, sondern ein Signal dafür.

\subsection{Lohndiskriminierung}

Von Lohndiskriminierung spricht man, wenn es zu einer schlechteren Entlohnung kommt, als es durch die Arbeitsleistung gerechtfertigt wäre (vgl. Franz 2006: 340). Lohndiskriminierungen betreffen hauptsächlich Frauen, ausländische oder ältere Arbeitnehmer/-innen. Zur Analyse von Lohndiskriminierung ist es notwendig zu unterscheiden, worauf Lohnunterschiede beruhen.

Einerseits gibt es Lohnunterschiede, die auf einer geringeren Humankapitalausstattung, einer geringeren Berufserfahrung bzw. auf einer geringeren Produktivität beruhen. So könnte etwa die Humankapitalausstattung von Frauen geringer 
sein, weil Unternehmen aufgrund unvollkommener Informationen über die erwartete Betriebszugehörigkeitsdauer weniger in die betriebliche Weiterbildung von Frauen investieren. Frauen erhalten infolge einen niedrigeren Lohn, da sie eine geringere Humankapitalausstattung haben.

Andererseits erhalten diskriminierte Personengruppen trotz gleicher oder vielleicht sogar höherer Produktivität einen niedrigeren Lohn. Für Frauen zeigt sich beispielsweise, dass trotz Kontrolle relevanter Determinanten von Lohnunterschieden Frauen einen geringeren Lohn bekommen, d.h. es herrscht Lohndiskriminierung vor (vgl. Franz 2006: 341).

Eine Möglichkeit, warum Frauen auch nach Kontrolle relevanter Determinanten niedrigeres Einkommen haben ist die sogenannte "statistische Diskriminierung“ (Franz 2006: 341). Sie beruht auf der Tatsache, dass Produktivität nur schlecht oder nicht vollständig messbar ist. Es liegen unvollständige Informationen bezüglich der wahren Produktivität vor. Organisationen benutzen bei der Festlegung des Lohns das Geschlecht zur Bestimmung der vermutlichen Produktivität, da die tatsächliche Produktivität unbekannt ist. Frauen wird in Folge eines Vorurteils nur eine geringere Produktivität zugetraut, weshalb sie ein geringeres Einkommen haben. Möglicherweise ist diese Art der Lohndiskriminierung nur vorübergehend vorhanden, wenn nämlich nach einer gewissen Zeit der Lohn angepasst wird, sobald die tatsächliche Produktivität bekannt ist. Für Frauen existieren Zugangsbeschränkungen für höherqualifizierte Arbeitsplätze, weil sie bei der Auswahl für diese Positionen von Männern diskriminiert werden. In der Gruppe der niedrigqualifizierten Arbeitnehmer/-innen sind überporportional viele Frauen, weshalb es zu Lohndifferenzialen kommt (vgl. Franz 2006: 342).

\subsection{Matching-Theorie}

Die Matching-Theorie kann theoretisch erklären, warum Löhne von Arbeitnehmer/-innen in vergleichbaren Tätigkeiten auf derselben Hierarchieebene nicht identisch sein müssen, sondern vielmehr teilweise erheblich differieren. Ausgangspunkt bildet die Annahme, dass Arbeitgeber bei der Personalauswahl und -zuordnung Entscheidungen unter Qualitätsunsicherheit treffen. Arbeitnehmer/-innen können einerseits bezüglich ihrer absoluten Qualität differieren. Darunter wird verstanden, dass die Leistungsfähigkeit eines Arbeitnehmers bzw. einer Arbeitnehmerin - also beispielsweise seine Produktivität oder ihr Talent unabhängig vom aktuellen betrieblichen Einsatzort der Person ist. Andererseits gibt es die relative Qualität eines Arbeitnehmers bzw. einer Arbeitnehmerin, die umschreibt, wie gut eine Person die spezifischen Anforderungen einer bestimmten Stelle im Vergleich zu anderen Arbeitnehmer/-inne/n erfüllt. Aussagen zur relativen Qualität eines Arbeitnehmers oder einer Arbeitnehmerin sind daher nur möglich, wenn die Anforderungen des konkreten betrieblichen Einsatzortes berücksichtigt werden. Mit zunehmender Dauer des Beschäftigungsverhältnisses 
erhalten sowohl Arbeitnehmer/-innen als auch Arbeitgeber/-innen zusätzliche Informationen über die tatsächliche Qualität eines Arbeitnehmers bzw. einer Arbeitnehmerin. Arbeitskräfte, deren Fähigkeiten und Eigenschaften optimal zu einer konkreten Stelle passen, sind auf dieser Stelle produktiver als jene, deren Qualitätsmerkmale nicht oder weniger gut mit den Anforderungen der Stelle übereinstimmen. Aufgrund dieses Produktivitätsvorteils erhalten Arbeitnehmer/innen in effizienten Zuordnungen (, matches') daher auch höhere Einkommen als andere Beschäftigte auf vergleichbaren Stellen.

Bei Gültigkeit der Matching-Theorie ist zu erwarten, dass Arbeitnehmer/-innen, die sich aufgrund ihrer hohen relativen Qualität in einem effizienten Match befinden, lange in ihrer aktuellen Tätigkeit verweilen und höhere Einkommen als andere Beschäftigte erhalten. Empirisch sollte daher ein positiver Zusammenhang zwischen der Verweildauer in einer Tätigkeit und dem Einkommen nachweisbar sein. Tatsächlich konnte empirisch dieser Zusammenhang jedoch nicht festgestellt werden. Vielmehr zeigt sich, dass sich die Verweildauer auf einer Hierarchiestufe nicht positiv auf die Lohnhöhe auswirkt (vgl. Becker 2000: 147ff.)

\subsection{Rent-Sharing Theorie}

Mitunter wird die Theorie auch Zahlungsfähigkeitshypothese oder Produktmarktmacht-Ansatz genannt. Die Rent-Sharing Theorie argumentiert, dass die Höhe der Renten ${ }^{12}$, die auf unvollkommenen Produktmärkten entstehen, die Lohnhöhe in einer Organisation beeinflussen (vgl. Klodt 2000, 20). Höhere Renten führen der Theorie zufolge zu einem höheren Lohnniveau in einer Organisation. Höhere Renten ermöglichen es einer Organisation, diese Renten mit den Arbeitnehmer/-innen zu teilen, d.h. höhere Löhne zu zahlen. Die Arbeitskräfte haben die Möglichkeit, über diese Renten zu verhandeln (vgl. Stephan 2001: 39ff.).

Unternehmen erwirtschaften in Abhängigkeit ihrer Größe und ihrer sektoraler Zugehörigkeit unterschiedlich hohe Renten und verfügen daher über unterschiedlich hohe Zahlungsfähigkeit in Bezug auf Löhne. Daraus ist nicht unmittelbar ersichtlich, warum erwirtschaftete Renten an Arbeitnehmer/-innen weitergegeben werden sollen.

Das am häufigsten vorgebrachte Argument dafür ist, dass Arbeitnehmer/-innen in irgendeiner Form über Verhandlungsmacht verfügen, die es ihnen erlaubt, sich einen Teil der Renten anzueignen. Für die Verhandlungsmacht der Arbeit-

12 Renten sind als „Extragewinne“ zu verstehen (Klodt 2000: 15). Die Theorie unterstellt, dass Unternehmen nicht den vollkommenen Wettbewerbsbedingungen unterliegen, bei denen es keine Renten gäbe. 
nehmer/-innen ist der gewerkschaftliche Organisationsgrad von Interesse. Arbeitnehmer/-innen müssen sich nicht explizit zu einer Gewerkschaft zusammenschließen, auch implizit kann sich eine Gruppe von Insidern zusammenschließen, die sich durch das Vorhandensein von firmenspezifischen Fähigkeiten auszeichnet. Insider besitzen Marktmacht, die aus Transaktionskosten resultiert. Einstellungen und Entlassungen verursachen Kosten, etwa durch Ausbildung und Einschulung. Mit höheren Löhnen könnte jedoch auch eine gewerkschaftliche Organisation verhindert werden wollen, da diese mit höheren Kosten für die Arbeitgeberseite verbunden wäre. Die Bildung einer Gewerkschaft soll durch freiwillige Zahlung von höherem Einkommen abgewendet werden.

Eine weitere mögliche Erklärung ist eine mögliche Interessendivergenz zwischen der Unternehmensführung einer Organisation und den Eigentümer/inne/n. Voraussetzung dafür sind Informationsasymmetrien zwischen den Anteilseigner/-inne/n und den Manager/-inne/n. Hier wird unterstellt, dass die Unternehmensführung nicht streng gewinnmaximierend handelt, sondern höhere Löhne zahlt, um sich die Loyalität unterstellter Arbeitnehmer/-innen zu sichern, selbst wenn dies nicht produktivitätssteigernd wirkt. Insbesondere wenn Renten erzielt werden, ist ein derartiges Verhalten möglich oder erforderlich (vgl. Klodt 2000: 20f.).

Ähnlich wie im Fall von Effizienzlöhnen ${ }^{13}$ kann als weiterer Erklärungsgrund argumentiert werden, dass sich das „Teilen“ der Renten mit den Arbeitnehmer/innen positiv auf die Produktivität und die Arbeit-nehmerfluktuation der Individuen auswirkt, und es deshalb vorteilhaft für das Unternehmen ist, Rent-Sharing zu betreiben (vgl. Piekkola, Kauhanen 2003). Analog zum Fairness-Ansatz der Effizienzlohntheorie kann auch die Hypothese aufgestellt werden, dass Renten aus ethischen bzw. Fairness-Überlegungen durch das Management mit der Belegschaft geteilt werden.

In empirischen Studien lässt sich ein statistisch gesicherter, positiver Zusammenhang zwischen der Profitabilität von Unternehmen und den gezahlten Einkommen belegen (vgl. z.B. Klodt 2000).

\subsection{Verhandlungstheorien}

Eng mit der Rent-Sharing Theorie zusammenhängend sind Verhandlungstheorien. Hier wird ausführlicher dargestellt, wie die Lohnhöhe von der Verhandlungsmacht der kollektiven Arbeitnehmer/-innen und der Arbeitgeber/-innen abhängt. Je höher die Marktmacht der Arbeitnehmer/-innen ist, desto höher ist 
die Lohnhöhe in einer Organisation (vgl. z.B. Franz 2006: 286ff., Metcalf et al. 2000).

Gewerkschaften, die auf kollektiver Ebene über Löhne verhandeln, bevorzugen Entlohnung nach dem Senioritätsprinzip, d.h. eine mit der Anstellungsdauer bzw. dem Alter steigende Entlohnung, im Vergleich zu einer Entlohnung, die sich an der individuellen Leistung orientiert (vgl. Metcalf et al. 2000: 2f. sowie $16) .^{14}$

\subsection{Effizienzlohntheorie und Theorie der relativen Position}

Die Grundidee der Effizienzlohntheorie ist, dass es für Organisationen vorteilhaft sein kann, Löhne über dem Markträumungsniveau zu bezahlen, da Löhne als ein Instrument zur Beeinflussung der Leistungsbereitschaft, Motivation, Betriebstreue und damit zur Produktivität der Arbeitnehmer/-innen verstanden werden (vgl. Landmann, Jerger 1999: 184). Dabei geht man wiederum von einem positiven Zusammenhang zwischen der Lohnhöhe und der Produktivität der Arbeitnehmer/-innen aus, die in Folge die Höhe des Outputs beeinflusst. Ein Absinken des Lohnsatzes löst eine Reduktion an Arbeitsanstrengung und folglich Produktivität der Arbeitnehmer/-innen aus, die die Einsparung der Lohnkosten überkompensieren würden.

Durch Effizienzlöhne wird Arbeitslosigkeit induziert, was aber von den Unternehmen akzeptiert wird, da sie die Disziplinierungswirkung von Effizienzlöhnen verstärkt. Arbeitslosigkeit erhöht die Opportunitätskosten des Verlusts des Arbeitsplatzes. Die Effizienzlohntheorie kann einen Erklärungsbeitrag leisten, warum es am Arbeitsmarkt dauerhaft positive Lohndifferenziale gibt.

$\mathrm{Zu}$ unterscheiden sind vier unterschiedliche Stränge der Effizienzlohntheorie (vgl. z.B. Landmann, Jerger 1999: 183ff., Becker 2000: 107f.):

a) Shirking-Ansatz: Hier wird unterstellt, dass Arbeitnehmer/-innen in der Lage sind, das Ausmaß ihrer Leistungsanstrengung zu variieren, ohne dass dies von der Unternehmensleitung vollkommen beobachtbar bzw. vertraglich festlegbar ist. Bezüglich der Leistungsintensität herrscht asymmetrische Information zwischen Arbeitgeber/-innen und Arbeitnehmer/-innen. Durchgängige Kontrolle zu jeder Zeit ist aufgrund zu hoher Kontrollkosten nicht möglich. Durch die Zahlung von Effizienzlöhnen, die über dem Markträumungsniveau liegen, soll ein gewisses Drohpotential des Arbeitsgebers gegenüber dem Arbeitnehmer bzw. der Arbeitnehmerin aufgebaut werden. Wenn nämlich Shirking, das in der

14 Vgl. auch Kapitel 10.2, in dem genauer auf den Effekt von kollektiven Lohnverhandlungen auf die Lohnstreuung eingegangen wird. 
deutschsprachigen Literatur auch als Bummeln bezeichnet wird, eines Arbeitnehmers bzw. einer Arbeitnehmerin entdeckt wird, wird die Person entlassen und muss sich einen neuen Arbeitgeber suchen, der womöglich nur mehr den markträumenden Lohnsatz zahlt. Deshalb werden höhere Löhne bezahlt, um shirking zu verhindern.

b) Turnover-Ansatz: Unternehmen zahlen bei diesem Ansatz aus dem Grund Effizienzlöhne, um die Fluktuationsrate der Belegschaft zu senken, da eine hohe Fluktuation mit Transaktionskosten verbunden ist. Aufgrund höherer Löhne werden für den Arbeitnehmer bzw. die Arbeitnehmerin die Opportunitätskosten eines Arbeitgeberwechsels erhöht.

c) Selektions-Ansatz: Dieser Ansatz, der auch als Adverse-Selection-Ansatz bezeichnet wird, geht davon aus, dass Effizienzlöhne gezahlt werden, um die durchschnittliche Qualität der Bewerber/-innen um eine vakante Stelle zu erhöhen. Der Arbeitgeber kann über Lohnofferte, die oberhalb des Lohnes für die Durchschnittsqualität liegen, sowohl die Anzahl der Stellenbewerber/-innen als auch die erwartete Qualität der eingestellten Bewerber/-innen positiv beeinflussen.

d) Gift-Exchange bzw. Fairness Ansatz: Hierbei wird ein über dem Markträumungsniveau liegender Lohn von den Arbeitnehmer/-innen als Geschenk seitens der Unternehmensleitung aufgefasst, das sie wiederum durch zusätzliche Leistungsanstrengungen vergelten.

Effizienzlöhne liegen in jenen Branchen besonders weit über dem Markträumungslohn, in denen hohe Überwachungskosten und eine hohe Arbeitnehmerfluktuation vorherrschen.

Hohe Überwachungskosten und eine hohe Arbeitnehmerfluktuation treffen vermutlich nicht auf alle Arbeitnehmer/-innen innerhalb einer Organisation zu. Deshalb stellt sich die Frage, ob in Folge nur bestimmte Arbeitnehmer/-innen innerhalb einer Organisation Effizienzlöhne erhalten (vgl. u.a. Landmann, Jerger 1999: 183ff.). Die Theorie der relativen Position geht davon aus, dass Normen über Gerechtigskeitsvorstellungen eine große Bedeutung für die Arbeitnehmer/innen haben, wodurch es sinnvoll sein kann, auch jenen Personen innerhalb einer Organisation Effizienzlöhne zu zahlen, die keine hohen Überwachungskosten oder keine hohe Arbeit-nehmerfluktuation haben (vgl. Gerlach, Stephan 2006b: 48f.). Ungleiche Bezahlung kann sonst zu Demotivation und einem Rückgang der Produktivität führen. ${ }^{15}$ 


\subsection{Sorting-by-abilities Theorie}

Diese Theorie stellt dar, warum manche Organisationen ein höheres Lohnniveau haben als andere (vgl. Kremer, Maskin 1996). Der Grund für Löhne, die über dem Markträumungsniveau liegen, wird bei dieser Theorie in der Qualifikation der Arbeitnehmer/-innen gesehen. Die Theorie geht davon aus, dass in einer einzelnen Organisation Personen mit ähnlichen Qualifikationen arbeiten. Es kommt zunehmend zu einer Trennung zwischen niedrig und hoch qualifizierten Arbeitnehmer/-innen zwischen Organisationen, d.h. innerhalb einer Organisation wird das Qualifikations-niveau der Arbeitnehmer/-innen ähnlicher. Die Streuung des Qualifikations-niveaus innerhalb einer Organisation und die damit verbundene innerbetriebliche Lohnstreuung gehen zurück. Einhergehend mit dieser Entwicklung ist ein Anstieg der Einkommensungleichheiten zwischen Organisationen.

\subsection{Kompensierende Lohnzahlungen}

Unternehmen sind auf kompetitiven Arbeitsmärkten gezwungen, ihre Arbeitnehmer/-innen für schlechte Arbeitsbedingungen zu entschädigen. Es wird davon ausgegangen, dass in die Nutzenfunktionen von Arbeitnehmer/-innen schlechte Arbeitsbedingungen negativ eingehen. Arbeitgeber müssen daher eine Lohnprämie zahlen, die sie für den Disnutzen aus den negativen Arbeitsbedingungen entschädigt. Der Begriff ,schlechte' Arbeitsbedingungen ist weit zu fassen, dazu können z.B. hohe Unfallrisiken, monotone Tätigkeiten, Schichtarbeit oder hohe körperliche oder psychische Belastungen durch die Arbeitsumgebung gezählt werden (vgl. Becker 2000: 118).

\subsection{Zusammenfassung der allgemeinen Theorien zur Bestimmung der Lohnhöhe}

Abbildung 1 gibt eine zusammenfassende Übersicht über die eben vorgestellten für NPO besonders relevanten Theorien, die die Lohnhöhe eines Individuums erklären können. Dabei wird zwischen Theorien, die Aussagen über die Lohnhöhe auf Individualebene treffen und jenen, die sich auf die betriebliche oder Branchenebene beziehen, unterschieden.

Die Theorien, die in der oberen Hälfte der Graphik abgebildet sind, erklären Lohnunterschiede zwischen Organisationen, wobei sich die einzelnen Theorien stark in dem unterscheiden, was sie genau zu erklären vermögen. Bei der Effizienzlohntheorie, der Rent-Sharing Theorie, bei den kompensierenden Lohnzahlungen wird davon ausgegangen, dass die Lohnpolitik des Unternehmens jeweils für alle Mitarbeiter/-innen gilt. Dies ist jedoch nicht zwingenderweise der Fall. Die Theorien in der unteren Hälfte der Graphik haben gemein, dass sie 
Lohnunterschiede auf (zum Teil angenommene) Produktivitätsunterschiede auf Individualebene zurückführen.

\section{Abbildung 1: Übersicht über relevante Erklärungsansätze}

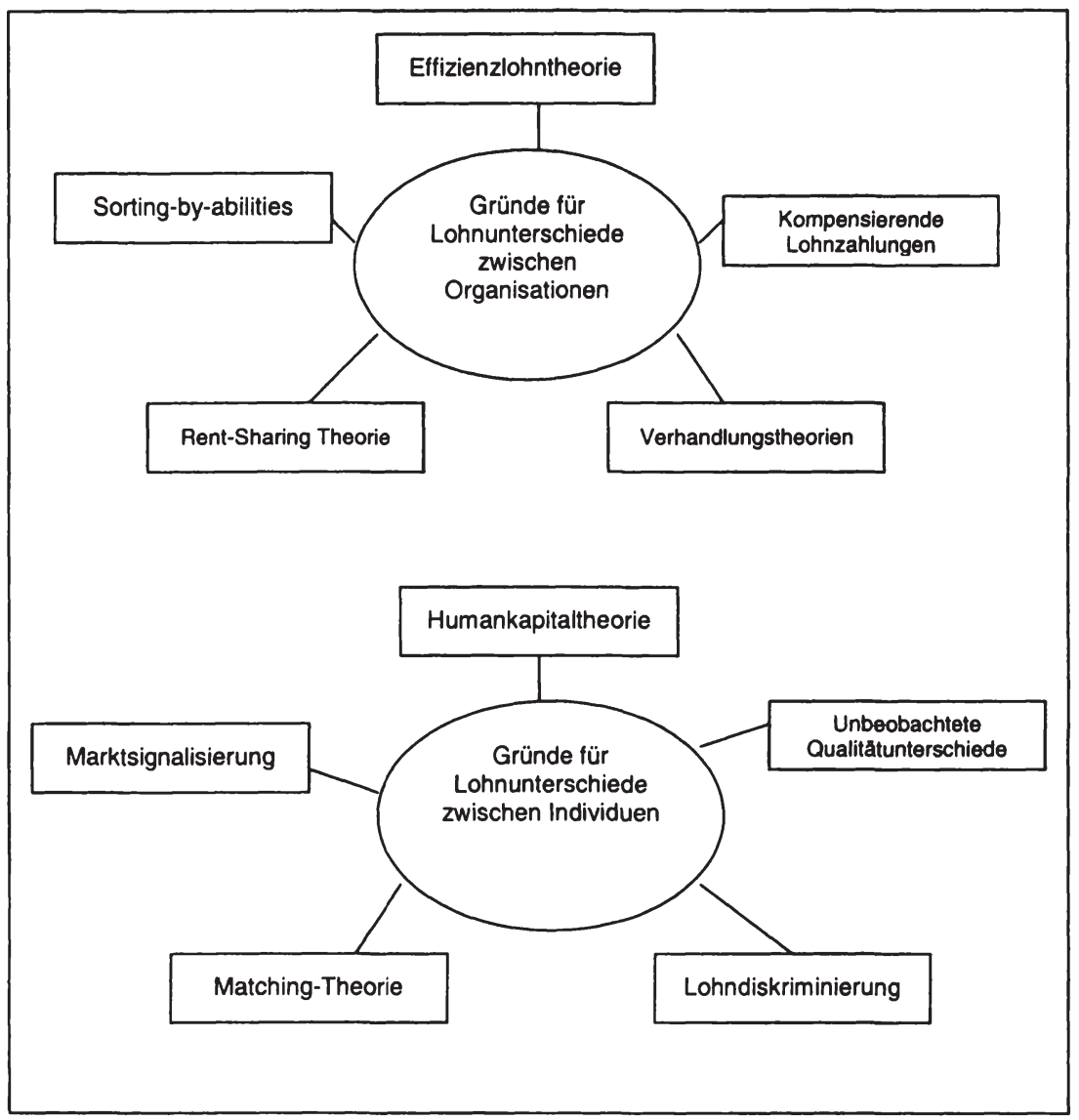

\section{Quelle: eigene Darstellung}

Die Hauptaussagen der einzelnen Theorien werden in der nachstehenden Tabelle kurz zusammengefasst: 


\section{Tabelle 1: Unterschiedliche Theorien zur Erklärung von Unterschieden in der Lohnhöhe}

\begin{tabular}{|c|c|}
\hline Theorie & Hauptaussage \\
\hline Humankapitaltheorie & $\begin{array}{l}\text { Löhne bestimmen sich durch unterschiedliche Ausbildung und Erfahrung, die zu } \\
\text { Produktivitätsunterschieden führen. }\end{array}$ \\
\hline $\begin{array}{l}\text { Theorie der unbeobachteten } \\
\text { Qualitätsunterschiede: }\end{array}$ & Unbeobachtbare Qualitätsunterschiede führen zu Produktivitätsunterschieden \\
\hline Lohndiskriminierung & $\begin{array}{l}\text { Die Organisation schreibt unterschiedliche Produktivität aufgrund verschiedener } \\
\text { persönlicher Charakteristika zu }\end{array}$ \\
\hline Matching-Theorie & $\begin{array}{l}\text { effiziente Zuordnungen zwischen Arbeitnehmer/-innen und Arbeitsplatz führen } \\
\text { durch Produktivitätssteigerungen zu höheren Löhnen }\end{array}$ \\
\hline Marktsignalisierung & Durch Ausbildung wird unterschiedliche Produktivität signalisiert \\
\hline Effizienzlohntheorie & $\begin{array}{l}\text { Es ist vorteilhaft für die Organisation, hohe Löhne zu zahlen, da dies die } \\
\text { Produktivität der Arbeitnehmer/-innen steigert }\end{array}$ \\
\hline $\begin{array}{l}\text { Kompensierende } \\
\text { Lohnzahlungen }\end{array}$ & $\begin{array}{l}\text { Überdurchschnittlich schlechte/gute Arbeitsbedingungen werden durch } \\
\text { überdurchschnittliche hohe/niedrige Löhne kompensiert }\end{array}$ \\
\hline Verhandlungstheorien & Hohe Verhandlungsmacht der Arbeitnehmer/-innen führt zu höheren Löhnen \\
\hline Rent-Sharing Theorie & Renten werden in Form höherer Löhne an die Arbeitnehmer/-innen verteilt \\
\hline Sorting by abilities & In Organisationen arbeiten Individuen mit ähnlicher Produktivität \\
\hline
\end{tabular}

Quelle: eigene Darstellung

\section{Besonderheiten in der Bestimmung der Lohnhöhe bei NPO}

Ausgehend von den oben vorgestellten allgemeinen Theorien zur Bestimmung der Lohnhöhe, werden in diesem Kapitel Besonderheiten von NPO dargestellt, die für die Bestimmung der Lohnhöhe von Arbeitnehmer/-inne/n in NPO von Bedeutung sein können. NPO in ihrer Eigenart als Organisationen zwischen Staat und Markt bringen besondere Eigenschaften und Voraussetzungen mit sich, die sich auch auf die Bedeutung der oben vorgestellten Theorien für den Nonprofit Sektor und damit auch auf die Lohnhöhe eines Arbeitnehmers bzw. einer Arbeitnehmerin in einer NPO auswirken können. Besonders ausführlich wird dabei auf ehrenamtliche, d.h. unbezahlte Arbeit und die Finanzierung von NPO eingegangen, die die personellen und finanziellen Ressourcen der Organisation erweitern. 


\subsection{Gewinnausschüttungsverbot von NPO}

NPO unterliegen einem Gewinnausschüttungsverbot ${ }^{16}$, d.h. sie dürfen vorhandene Gewinne nicht an Eigentümer/-innen oder Mitglieder verteilen. Sehr wohl dürfen NPO jedoch Gewinne erzielen (vgl. z.B. Badelt et al. 2007b: 7). Sie müssen jedoch in der Organisation verbleiben und für die Mission der NPO verwendet werden. Der Name nonprofit, auch not-for-profit impliziert auch, dass NPO nicht existieren, um Gewinne zu erwirtschaften bzw. zu maximieren. Nicht die Gewinnmaximierung steht im Mittelpunkt des Interesses, sondern ein Sachziel, nämlich die Zweckausrichtung der Organisation bzw. die Mission (vgl. Heitzmann 2001: 33). ${ }^{17}$

Das Gewinnausschüttungsverbot und die fehlende Gewinnmaximierung als Formalziel kann auch die Lohnhöhe von Beschäftigten einer NPO beeinflussen. Insbesondere könnte dadurch die Rent-Sharing Theorie, die weiter oben dargestellt wurde, für NPO zu adaptieren sein.

Die Tatsache, dass NPO keine Gewinne an Eigentümer/-innen oder Mitglieder ausschütten dürfen, kann sich sowohl positiv als auch negativ auf die Lohnhöhe der Arbeitnehmer/-innen auswirken.

Aus der Rent-Sharing Theorie kann abgeleitet werden, dass NPO möglicherweise höhere Löhne zahlen, weil sie dem Gewinnausschüttungsverbot unterliegen. Dieses kann dazu führen, dass Manager/-innen keinen Anreiz für eine Lohnsenkung haben, da dadurch resultierende höhere Gewinne nicht an externe Stakeholder ausgeschüttet werden dürfen. NPO haben also keinen gewinngetriebenen Anreiz, durch niedrige Löhne Kosten zu senken, was in der Literatur auch als philantropic wage-setting (Feldstein 1971) bzw. attenuated property rights-Phänomen (Borjas et al. 1983) bezeichnet wird. Lohnzahlungen, die über dem Markträumungsniveau liegen, können als karitative Handlung an Arbeitnehmer/-innen der NPO interpretiert werden. Die Rent-Sharing Theorie könnte demnach in NPO eine besondere Rolle spielen. Manager/-innen könnten auch Renten verteilen, um den eigenen Nutzen zu maximieren, indem sie sich selbst und ihren Mitarbeiter/-inne/n höhere Löhne zahlen. Nach der Agency-Theorie werden Manager/-innen der NPO nicht in gleichem Maße wie jene einer gewinnorientierten Firma von Eigentümer/-inne/n überwacht, was dazu führt,

16 Für eine kritische Betrachtung des Gewinnausschüttungsverbots und dessen Folgen siehe Valentinov (2008).

17 Eine ausführliche Diskussion, welche Zielorientierung NPO haben, findet sich beispielsweise in James, Rose-Ackerman (1986). Ben-Ner (1986: 99) unterstellt NPO das Ziel der Maximierung der Konsumentenrente unter der Nebenbedingung des NullProfits. Brown und Slivinski (2006: 140f.) diskutieren die Folgen, die sich durch eine der Gewinnorientierung unterschiedlichen Zielorientierung ergeben. 
dass sie Renten verteilen und höhere Löhne bezahlen können (vgl. Speckbacher 2003: 272).

Umgekehrt führt die fehlende Gewinnorientierung vielleicht auch dazu, dass gar keine Renten oder weniger hohe Renten entstehen. In diesem Fall wäre die Rent-Sharing Theorie für NPO irrelevant, da keine Renten vorhanden sind, die durch Lohnzahlungen über dem Markträumungsniveau ausgeschüttet werden können. Eine Missionsorientierung der Organisation kann auch dazu führen, dass die Organisation zu Gunsten von Sachzielen bzw. zu Gunsten der Mission, Kosten senken will und daher erwirtschaftete Renten nicht an die Belegschaft verteilt, sondern in die Organisation reinvestiert.

Lakdawalla und Philipson (1998) führen die Hypothese an, dass NPO hauptsächlich in hoch kompetitiven Branchen entstehen, da der Nachteil des Gewinnausschüttungsverbots in Branchen, in denen aufgrund des hohen Wettbewerbs nur wenige Renten erwirtschaftet werden können, weniger schwer wiegt. Anders gesagt, werden sich Organisationen eher dann dazu entscheiden, als gewinnorientierte Firma aufzutreten, wenn in der Branche hohe Gewinne zu erwarten sind. Eine empirische Überprüfung der Hypothese erfolgt jedoch nicht, und es ist besonders für Österreich fraglich, ob die Hypothese zutrifft.

Aufgrund des Gewinnausschüttungsverbots können NPO Güter oder Dienstleistungen mit höherer Qualität produzieren, da sie wenig Anreiz haben, bei der Qualität zu ,schummeln“, um Kosten zu sparen. Sobald die Qualität eines Produkts oder einer Dienstleistung schwer überprüfbar ist entsteht ein MoralHazard Problem ${ }^{18}$, da die Firmen einen Anreiz haben, die Qualität für die Kund/-inn/en unbemerkt zu senken und Kosten zu sparen (vgl. z.B. Akerlof 1970 und Hansmann 1980, der das Problem für NPO beschreibt). Als Folge wird von gewinnorientierten Unternehmen der Markt für Produkte mit hoher Qualität nicht bedient. NPO haben, wie oben beschrieben, weniger bzw. keinen Anreiz bei der Qualität zu schummeln und werden von Kund/-inn/en als vertrauenswürdiger eingestuft. Als Folge einer hohen angebotenen Qualität der Produkte und Dienstleistungen, suchen NPO als Arbeitgeber auch vorwiegend hoch qualifizierte Arbeitnehmer/-innen (vgl. James, Rose-Ackerman 1986, 41). Der Selektions-Ansatz der Effizienzlohntheorie besagt, dass hohe Löhne aus dem Grund gezahlt werden, um die Durchschnittsqualität der Bewerber/-innen um eine Stelle zu erhöhen. Wenn also NPO besonders hohe Qualität anbieten wollen und aus diesem Grund besonders qualifizierte Arbeitskräfte suchen, so kann der Selektions-Ansatz für NPO eine wichtige Rolle spielen.

18 ,Moral Hazard' liegt vor, wenn ,...eine Partei, deren Handlungen unbeobachtet bleiben, die Wahrscheinlichkeit oder das Ausmaß einer Zahlung beeinflussen kann" (Pindyck, Rubinfeld 2005: 814). 


\subsection{Mission und Produkt der NPO}

NPO können sich von gewinnorientierten Unternehmen durch ihre Tätigkeit unterscheiden. Dabei wird die Annahme getroffen, dass sich das Produkt oder die Dienstleistung der NPO von dem oder der des gewinnorientierten Unternehmens unterscheidet. Wegen der Besonderheit des Produkts wird unterstellt, dass Arbeitnehmer/-innen bereit sind, auf einen Teil des Lohns zu verzichten. Diese Hypothese wird auch Arbeitsspendenhypothese genannt. Nach dieser beziehen Arbeitnehmer/-innen in NPO niedrigere Löhne (vgl. z.B.: Frank 1996; Leete 2006; Hansmann 1980; Preston 1989; Rose-Ackermann 1986; Ruhm, Borkoski 2003). Obwohl verschiedene Autor/-inn/en die Theorie leicht variieren, bleibt die Hauptaussage der Theorie dieselbe: Arbeitnehmer/-innen „spenden“ einen Teil ihrer Arbeitszeit, d.h. sie akzeptieren niedrigere Löhne, da sie die Möglichkeit haben, an einer in ihren Augen sozial wertvollen Tätigkeit der NPO mitzuwirken.

Das produzierte Gut bzw. die produzierte Dienstleistung ist Preston (1989) zufolge ein Gut mit positiven sozialen Externalitäten, nach Frank (1996: 2) eines, das zu „moralischer Befriedigung“ führt. Das Ziel einer NPO ist die Erfüllung einer Mission, die einen positiven sozialen Nutzen darstellt. Die Arbeitnehmer/-innen einer NPO wissen, dass sie zur Verwirklichung eines idealistischen Ziels beitragen und nicht für den Profit eines Kapitaleigners bzw. einer Kapitaleignerin arbeiten (Rose-Ackermann 1986). Dadurch wird unterstellt, dass Mitarbeiter/-innen in NPO außergewöhnlich hoch intrinsisch motiviert sind.

Mosca, Musella und Pastore (2007: 58) unterstellen, dass zusätzlich zum produzierten Output der NPO in Form eines Produkts oder einer Dienstleistung aufgrund der Besonderheit des Outputs „Beziehungsgüter“ erstellt werden, d.h. dass Beziehungen, Freundschaften oder Vertrauen zwischen Arbeitnehmer/-innen oder zwischen Arbeitnehmer/-innen und Stakeholdern entstehen. Diese Beziehungsgüter können nur durch die Arbeit in der NPO konsumiert werden, wobei mehr Konsum durch mehr Arbeit entsteht. Benz (2005) vergleicht die Arbeitszufriedenheit von Arbeitnehmer/-innen in NPO und gewinnorientierten Unternehmen und findet für die erste Gruppe höhere Arbeitszufriedenheit. Es wird die Schlussfolgerung aufgestellt, dass die Arbeit in NPO höheren intrinsischen Nutzen bietet. Zur gleichen Schlussfolgerung gelangen Lanfranchi und Narcy (2008), die ebenfalls die Arbeitszufriedenheit im Nonprofit Sektor untersuchen und feststellen, dass Arbeitsplätze in NPO so konzipiert sind, dass sie die hohe intrinsische Motivation der Arbeitnehmer/-innen unterstuitzen.

Auch das fehlende Gewinnmaximierungsziel kann darauf hindeuten, dass das primäre Motiv einer Person, in einer NPO zu arbeiten, ein nicht-monetäres sein könnte, also etwa ein soziales oder ethnisches Ziel. Als Folge ist der Lohn als 
Motivationskriterium weniger relevant als in gewinnorientierten Unternehmen (vgl. Brandl et al. 2006: 357).

Dies kann dazu führen, dass Effizienzlöhne im Nonprofit Sektor weniger wichtig bzw. unwirksamer sind, da die Zahlung von Geld die Motivation und Produktivität von Beschäftigten im Nonprofit Sektor weniger stark beeinflussen kann. Die außergewöhnlich hohe Motivation und Identifikation kann dazu führen, dass Effizienzlöhne als zusätzlicher Motivationsfaktor, um shirking zu verhindern, für Arbeitnehmer/-innen in NPO an Bedeutung verlieren. Der ShirkingAnsatz der Effizienzlohntheorie, der besagt, dass höhere Löhne gezahlt werden, um Bummeln zu verhindern, wird in diesem Fall bedeutungslos. Nach Mirvis und Hackett (1983) geben Arbeitnehmer/-innen im Nonprofit Sektor sehr viel öfter an, dass ihnen die Arbeit an sich wichtiger ist, als das Geld, das sie verdienen. Es besteht die Gefahr, dass durch extrinsische Motivation, d.h. z.B. durch die Zahlung von Effizienzlöhnen oder durch höhere Überwachung der Arbeit, die intrinsische verdrängt wird (vgl. z.B. Grepperud, Pedersen 2006; James Jr. 2005).

Die Arbeitsspendenhypothese wird besonders für Manager/-innen von NPO als bedeutsam angesehen, da sie vermehrt den tatsächlichen sozialen Nutzen der Aktivität einer NPO abschätzen können.

Allerdings muss auch bedacht werden, dass durch Organisationswachstum und durch eine zunehmende Professionalisierung von NPO auch Spezialist/-inn/en eingestellt werden, die eher extrinsisch motiviert sein können und sich weniger stark mit der NPO identifizieren. Für sie gilt diese Besonderheit weniger.

Im Widerspruch zur eben aufgestellten Hypothese steht die Überlegung, dass Effizienzlöhne insofern für NPO von besonderer Bedeutung sind, als in NPO häufig Tätigkeiten ausgeführt werden, die schwer oder gar nicht zu überwachen sind. Nach der Effizienzlohntheorie müsste eine NPO demnach höhere Löhne zahlen (vgl. Ito, Domain 1987).

Die Tatsache, dass NPO zumeist ein Ziel verfolgen, das dem Gemeinwohl dient, führt auch dazu, dass den Organisationen zum Teil besondere Erwartungen entgegengebracht werden. NPO werden als Ausdruck des Solidaritäts- bzw. des Gemeinschaftsgedankens gesehen (vgl. Badelt et al. 2007a: 522f.). Auch das kann das Lohnsetzungsverhalten von NPO beeinflussen. So wird einer Untersuchung von Brandl et al. (2006: 364) zufolge, das Senioritätsprinzip in österreichischen NPO favorisiert, sodass individuelle Lohnverhandlungen eine weniger große Rolle spielen. Leistungsbezogene Vergütung für Führungs- und gehobene Fachkräfte wird überwiegend abgelehnt (vgl. Brandl et al. 2006), da ein an individuelle Leistungen angelehntes Entgelt, mit fixen und variablen Gehaltsbestandteilen als wirkungslos, nicht messbar und die Identität einer NPO untergrabend angesehen wird (vgl. Brandl et al. 2006: 368). Unterstellt man NPO besondere Einstellungen zu Gleichheit und Gemeinschaft, so kann auch 
der Gift-Exchange bzw. Fairness Ansatz der Effizienzlohntheorie eine besondere Rolle spielen. Hierbei werden Effizienzlöhne von den Arbeitgebern gezahlt, die von den Arbeitnehmer/-inne/n als Geschenk aufgefasst werden. Effizienzlöhne können dann als Ausdruck des Solidaritätsgedankens interpretiert werden. Diese wiederum vergelten dies durch zusätzliche Leistungsanstrengung.

Sieht man NPO als Ausdruck des Solidaritätsgedankens in der Gesellschaft, so kann daraus auch die Hypothese abgeleitet werden, dass Lohndiskriminierung in NPO weniger häufig auftritt oder dass in NPO auch Arbeitnehmer/-innen beschäftigt werden, die aus diversen Gründen vielleicht am Arbeitsmarkt gewinnorientierter Unternehmen keine Beschäftigung finden.

Betrachtet man die Situation in Österreich, auf, dass NPO hauptsächlich in Dienstleistungsbranchen agieren. Personen, die in NPO arbeiten, sind hauptsächlich in den Branchen ,öffentliche Verwaltung, Landesverteidigung, Sozialversicherung“, „Unterrichtswesen“, „Gesundheits-, Veterinär- und Sozialwesen“ und „Erbringung von sonstigen öffentlichen und persönlichen Dienstleistungen“ tätig. ${ }^{19}$ Die Lohnhöhe in diesen Branchen ist, wie aus Tabelle 2 ersichtlich, im Vergleich eher gering (vgl. Statistik Austria 2007: 235). Betrachtet man österreichische Durch-schnittslöhne getrennt nach Geschlecht und ÖNACE, so zeigt sich, dass die Löhne in den Sektoren „Unterrichtswesen“, „Gesundheits-, Veterinär- und Sozialwesen“, „Erbringung von sonstigen öffentlichen und persönlichen Dienstleistungen“ unter den Durchschnittslöhnen über alle Branchen liegen. Eine Ausnahme stellen jedoch zum Teil weibliche Erwerbstätige in den Sektoren „Unterrichtswesen“ und „Gesundheits-, Veterinär- und Sozialwesen“ dar. In diesen Branchen liegen die Löhne für Frauen über dem Durchschnitt der Löhne für Frauen. Die Löhne für Tätige im Bereich „öffentliche Verwaltung, Landesverteidigung, Sozialversicherung“ liegen knapp über dem Durchschnitt über alle Branchen.

Das erwartete Lohnniveau in den Branchen, in denen NPO aktiv sind, ist im Vergleich zu anderen Sektoren niedrig. Ein Grund dafür können kleinere Renten in diesen Sektoren sein, die in Folge auch nicht durch Rent-Sharing an die Arbeitnehmer/-innen verteilt werden können.

19 Für eine Übersicht über die einzelnen ÖNACE Kategorien und einer Erläuterung zu den einzelnen Kategorien siehe: Statistik Austria (o.J.). 
Bruttoverdienste der unselbständigen Erwerbstätigen 2005 nach Abschnitten und Unterabschnitten der ÖNACE 1995

\begin{tabular}{|c|c|c|c|c|c|c|c|c|c|c|}
\hline \multirow{3}{*}{ ÖNACE } & \multirow{3}{*}{ Bezeichnung des Sektors } & \multicolumn{3}{|c|}{ Insgesamt } & \multicolumn{3}{|c|}{ Männer } & \multicolumn{3}{|c|}{ Frauen } \\
\hline & & $25 \%$ & $50 \%$ & $75 \%$ & $25 \%$ & $50 \%$ & $75 \%$ & $25 \%$ & $50 \%$ & $75 \%$ \\
\hline & & \multicolumn{9}{|c|}{ verdienen pro Monat weniger als. } \\
\hline$A, B$ & Land- und Forstwirtschaft, Fischerei und Fischzucht & 787 & 1269 & 1843 & 898 & 1412 & 2048 & 655 & 1065 & 1445 \\
\hline $\mathrm{C}$ & Bergbau und Gewinnung von Steinen und Erden & 2166 & 2764 & 3609 & 2296 & 2833 & 3675 & 1179 & 1953 & 3112 \\
\hline DA-DN & Sachgütererzeugung I & 1761 & 2344 & 3160 & 2040 & 2597 & 3417 & 1284 & 1709 & 2282 \\
\hline E & Energie- und Wasserversorgung & 2319 & 3414 & * & 2601 & 3685 & * & 1439 & 2225 & 3243 \\
\hline $\mathrm{F}$ & Bauwesen & 1838 & 2231 & 2730 & 1914 & 2279 & 2780 & 1050 & 1588 & 2230 \\
\hline G & $\begin{array}{l}\text { Handel; Instandhaltung u. Reparatur v. } \\
\text { Kraftfahrzeugen u. Gebrauchsgütern }\end{array}$ & 1252 & 1773 & 2490 & 1732 & 2220 & 3040 & 1019 & 1382 & 1896 \\
\hline $\mathrm{H}$ & Beherbergungs-und Gaststättenwesen & 943 & 1357 & 1749 & 1138 & 1516 & 1952 & 858 & 1272 & 1623 \\
\hline I & Verkehr und Nachrichtenübermittlung & 1544 & 2028 & 2556 & 1669 & 2122 & 2652 & 1205 & 1726 & 2263 \\
\hline $\mathrm{J}$ & Kredit- und Versicherungswesen & 1937 & 2958 & 4056 & 2559 & 3688 & * & 1587 & 2335 & 3263 \\
\hline $\mathrm{K}$ & $\begin{array}{l}\text { Realitätenwesen, Vermietung beweglicher Sachen, } \\
\text { Erbringung von unternehmensbezogenen } \\
\text { Dienstleistungen }\end{array}$ & 1259 & 1847 & 2658 & 1602 & 2089 & 3098 & 980 & 1479 & 2199 \\
\hline $\mathrm{L}$ & $\begin{array}{l}\text { Öffentliche Verwaltung, Landesverteidigung, } \\
\text { Sozialversicherung }\end{array}$ & 1519 & 2065 & 2656 & 1874 & 2365 & 2996 & 1329 & 1889 & 2468 \\
\hline$M$ & Unterrichtswesen & 981 & 1853 & 2623 & 1201 & 2119 & 3111 & 882 & 1686 & 2363 \\
\hline $\mathrm{N}$ & Gesundheits-, Veterinär- und Sozialwesen & 1113 & 1693 & 2411 & 1488 & 2265 & 3091 & 1055 & 1567 & 2220 \\
\hline 0 & $\begin{array}{l}\text { Erbringung v. sonstigen öffentlichen u. } \\
\text { persönlichen Dienstleistungen }\end{array}$ & 1092 & 1603 & 2445 & 1356 & 2082 & 3110 & 991 & 1391 & 1993 \\
\hline $\mathbf{P}$ & Private Haushalte & 635 & 961 & 1470 & 589 & 1091 & 2119 & 638 & 949 & 1425 \\
\hline$Q$ & Exterritoriale Organisationen und Körperschaften & 1585 & 2380 & 3204 & 1751 & 2507 & 3118 & 1437 & 2325 & 3280 \\
\hline Zusamme & & 1390 & 2008 & 2762 & 1806 & 2344 & 3194 & 1090 & 1573 & 2212 \\
\hline
\end{tabular}

* Aufgrund der Höchstbeitragsgrundlage 2005 von $3630 €$ ergibt sich in diesen Fällen eine ausweisbare Obergrenze von $4235 €$ (inkl. Sonderzahlungen). Quelle: Statistik Austria (2007): Statistisches Jahrbuch Österreichs 2007: 235 
Aus der vorhin aufgestellten Vermutung, dass NPO besonders hohe Qualität anbieten und deshalb besonders Personen mit hoher Qualifikation anstellen, lässt sich der Schluss ziehen, dass in NPO Individuen mit überdurchschnittlich hohem Humankapital arbeiten. Die zu erwartende Lohnhöhe für Individuen in NPO würde in Folge im Vergleich zu anderen Sektoren überdurchschnittlich hoch sein.

\subsection{Arbeitsbedingungen in der NPO}

Unter Umständen herrschen in NPO andere Arbeitsbedingungen als in gewinnorientierten oder öffentlichen Unternehmen. So schreibt beispielsweise Rose-Ackerman (1996) NPO höhere Löhne zu, die auf einer durchschnittlich höheren Organisationsgröße beruhen. Für die USA gilt, dass NPO in den relevanten Sektoren, wie Kinderbetreuung, Pflege, Krankenhäuser und soziale Dienstleistungen größer sind als gewinnorientierte Unternehmen (vgl. RoseAckerman 1996). Es ist vielfach erwiesen, dass die Lohnhöhe und die Firmengröße aus mehreren Gründen - wie beispielsweise monotonere Arbeit in großen Firmen - positiv miteinander korrelieren (vgl. z.B. Haltiwanger, Davis 1996; Lallemand et al. 2005; Medoff, Brown 1989; Oi, Idson 1999). Auch für NPO wurde dieser Zusammenhang insbesondere für Manager/-innen/gehälter festgestellt (vgl. Hallock 2002; Oster 1998). Für Österreich bleibt zu prüfen, ob sich die Organisationsgröße von NPO in den einzelnen Branchen von jenen öffentlicher oder gewinnorientierter Unternehmen unterscheidet.

Preston (1989) zeigt, dass Beschäftigte im Nonprofit Sektor mehr Arbeitsautonomie, höhere Abwechslung in der Tätigkeit und höhere Einwirkungsmöglichkeiten bei der Arbeit haben, was zu vergleichweise niedrigeren Löhnen in NPO führt. Arbeitnehmer/-innen im Nonprofit Sektor erhalten außerdem mehr nichtmonetäre Nebenleistungen, was ebenso zu niedrigeren Löhnen führt (vgl. Benz 2005: 171). Auch Lanfranchi und Narcy (2008) zeigen in einer über sieben europäische Länder vergleichenden Studie, dass die Arbeitszufriedenheit durch nicht-pekuniäre Vorteile wie Eigenständigkeit, Ermessensfreiheit und nicht-monotone Tätigkeiten im Nonprofit Sektor höher ist.

\subsection{Gewerkschaftliche Organisation der Arbeitnehmer/-innen in einer NPO}

Eine weitere Besonderheit für Österreich ist, dass die Branchenkollektivvertragsdichte im österreichischen Nonprofit Sektor eher gering ist, was auch daran liegt, dass viele NPO die Voraussetzungen der Kollektivvertragsfähigkeit des Arbeitgebers nicht erfüllen (vgl. Runggaldier, Drs 2007: 314). Allerdings liegt für Österreich kein Zahlenmaterial vor, das genaue Angaben über die 
kollektivvertragliche Abdeckung von Arbeitnehmer/-innen im Nonprofit Sektor in Österreich macht. Die Organisationen im verwendeten „NPO-SteuerDatensatz $^{\text {‘20 }}$ unterliegen etwa $\mathrm{zu}$ einem Drittel einem Kollektivvertrag, ein weiteres Drittel orientiert sich beim Lohnschema an einem Kollektivvertrag. NPO haben durch die geringe kollektivvertragliche Abdeckung bei der Vertragsgestaltung bezüglich der Vergütung mehr Freiheiten. Dies lässt darauf schließen, dass Verhandlungstheorien, die Aussagen über den Einfluss kollektiver Arbeitgeber- und Arbeitnehmerinteressen auf die Lohnhöhe treffen, für NPO weniger relevant sind. Die Macht von Gewerkschaften bzw. eine andere Form von Verhandlungsmacht kollektiver Arbeitnehmer/-innen ist nicht stark ausgeprägt, was sich negativ auf die Lohnhöhe von Beschäftigten in NPO auswirken kann. Auch wenn es darum geht, überschüssige Renten an Arbeitnehmer/-innen zu verteilen, könnte sich die fehlende kollektive Verhandlungsmacht seitens der Beschäftigten negativ auswirken.

Allerdings spricht einiges dafür, dass die Verhandlungsmacht der kollektiven Arbeitnehmer/-innen des Nonprofit Sektors im Zeitablauf zunehmend ist. So wurde 2003 erstmals im Bereich der Gesundheits- und Sozialberufe ein Kollektivvertrag eingeführt, 2004 und 2005 Kollektivverträge für die außeruniversitäre Forschung und für Be-schäftigte in privaten Bildungseinrichtungen (vgl. Bönisch 2008: 207). Auch sonst lehnen sich NPO bei der Höhe der Vergütung auch ohne vorhandene Kollektivverträge häufig an Kollektivvertragssätze an, zum Teil auch, weil öffentliche Subventions- und Auftraggeber/-innen dies verlangen. Leistungsverträge werden zum größten Teil mit der öffentlichen Hand geschlossen, die als monopsonistischer Vertrags-partner gesehen werden kann. Ein von Eckardstein (2007: 289) genannter Grund für die Anpassung an andere Lohnschemen ist die höhere Legitimation der Vergütungshöhe gegenüber ihren Arbeitnehmer/-inne/n und externen Stakeholder/-inne/n.

\subsection{Ehrenamtliche Beschäftigung ${ }^{21}$}

Betrachtet man das Ausmaß von ehrenamtlicher Tätigkeit in einer Volkswirtschaft, so wird schnell klar, dass sie ein nicht zu verachtendes Arbeitsinput darstellt. So wurde beispielsweise für die USA geschätzt, dass ca. 83,9 Millionen Erwachsene einer ehrenamtlichen Tätigkeit nachgehen, was $44 \%$ aller Erwachsenen in der USA darstellt. Von diesen Ehrenamtlichen arbeiten ca. 67\% für eine NPO (vgl. Leete 2006: 167). Für Europa schwankt der Anteil der Erwachsenen, die sich ehrenamtlich betätigen, zwischen $11 \%$ und $34 \%$ nach einer Schätzung von Gaskin and Smith (vgl. 1995: 28), die zehn europäische

Vgl. Kapitel 7.3.

21 In dieser Arbeit werden die Begriffe Freiwilligenarbeit und Ehrenamt gleich gesetzt. 
Länder analysieren. In Österreich sind, einer Studie von Badelt und Hollerweger (2007) zufolge, 51,1\% aller Österreicher über 15 Jahren ,im weitesten Sinn“ ehrenamtlich tätig, das sind mehr als 3,1 Millionen Personen (Badelt, Hollerweger 2007: 508). Beschränkt man ehrenamtliche Tätigkeit auf freiwillige Arbeit innerhalb einer Organisation, liegt das Arbeits-volumen, das durch ehrenamtliche Tätigkeit geleistet wird, bei 222.000 Vollzeitäquivalenten, wobei nach Ansicht von Badelt und Hollerweger der Nonprofit Sektor den größten Anteil hat (vgl. Badelt, Hollerweger 2007). Ehrenamtliche Tätigkeit findet sich freilich vereinzelt auch in gewinnorientierten Firmen, der Hauptanteil freiwilliger Arbeit innerhalb formeller Organisation ist jedoch NPO zuzurechnen. Im Vergleich zwischen NPO und gewinnorientierten Unternehmen in relevanten Branchen weisen NPO eine deutlich höhere Anzahl Freiwilliger auf (vgl. Menchik, Weisbrod 1987: 159, Leete 2006: 167).

Ehrenamtliche Mitarbeiter/-innen sind häufig bei der Produktion von Gütern mit positiven sozialen Externalitäten wie z.B. im Bereich des Gesundheits- und Bildungswesens beteiligt und sind für Anbieter aus dem Nonprofit Sektor maßgeblich (vgl. Menchik, Weisbrod 1987: 159). Die Beziehung zwischen den bezahlten und den unbezahlten Arbeit-nehmer/-inne/n ist komplex, da ehrenamtliche Mitarbeiter/-innen sehr unterschiedliche Aufgaben innerhalb der Organisation übernehmen können. Die Aufgaben der freiwilligen Mitarbeiter/innen unterscheiden sich dabei nicht notwendigerweise von denen der bezahlten Arbeitskräfte (vgl. Netting et al. 2005: 192). Ehrenamtliche Beschäftigte können daher, je nach Tätigkeit, entweder als Komplimente oder als Substitute zu bezahlten Arbeitskräften gesehen werden (vgl. Emanuele 1996; Handy et al. 2008). Es gibt, einer Reihe an Untersuchungen durch Handy, Mook und Quarter (2008) zufolge, auch keinen Hinweis darauf, dass ehrenamtliche Mitarbeiter/innen vermehrt durch bezahlte Arbeits-kräfte ersetzt werden oder umgekehrt.

Interessanterweise fanden Ehrenamtliche bei der Analyse von Löhnen in NPO, meines Wissens nach, bislang noch keine Beachtung, obwohl, wie in Folge dargestellt wird, einige Überlegungen dafür sprechen, dass das Vorhandensein von Ehrenamtlichen die Lohnhöhe von bezahlten Mitarbeiter/-innen in NPO beeinflussen kann. Ein Grund für die bisherige Missachtung eines möglichen Einflusses von ehrenamtlicher Tätigkeit auf die Lohnhöhe von bezahlten Beschäftigten in NPO ist das Fehlen geeigneter Daten. Lange Zeit wurden entweder ausschließlich Organisationsdaten oder Daten auf Individualebene zur Schätzung von Lohnfunktionen verwendet. Nun ist es natürlich möglich, Informationen über das Vorhandensein und die Anzahl Ehrenamtlicher in einer Organisation in einen Organisationsdatensatz $\mathrm{zu}$ inkludieren. Fragen über freiwillige Tätigkeit sind jedoch häufig nicht in branchen-übergreifenden Umfragen enthalten, da sie für die meisten Sektoren keine Relevanz haben. Außerdem sind die statistischen Ämter in vielen Ländern noch kaum an Daten zu Freiwilligenarbeit interes- 
siert. ${ }^{22}$ Bei der Analyse von Sekundärdaten ist es folglich nur schwer möglich, ehrenamtliche Tätigkeit als Variable in die Lohnfunktion mit ein-zubeziehen. Untersucht man Individualdaten, so gibt es zwar des Öfteren Informationen zu Einkommen und zu ehrenamtlicher Tätigkeit, jedoch bezieht sich letztere dann auf die ehrenamtliche Tätigkeit der befragten Person, d.h. wir wissen nichts darüber, ob es an dem Arbeitsplatz, an dem die befragte Person arbeitet, auch mitarbeitende Ehrenamtliche gibt.

Zuletzt wurden LEE-Daten entwickelt, einige Datensätze beinhalten NPO, wobei sie bislang nur von wenigen Autor/-inn/en genutzt wurden, um die Lohnhöhe in NPO bzw. das Lohndifferenzial zwischen NPO und gewinnorientierten Unternehmen zu bestimmen (vgl. z.B. Mocan, Tekin 2003, Mocan, Viola 1997). Ehrenamtliche wurden bisher auch bei Verwendung derartiger Daten noch nicht in die Schätzung der Lohngleichung aufgenommen.

Wie können ehrenamtliche Mitarbeiter/-innen einer NPO Löhne von bezahlten Beschäftigten beeinflussen? An dieser Stelle werden fünf Argumente dargestellt, warum sie in die Lohnfunktion von Arbeitnehmer/-innen einer NPO aufgenommen werden sollen:

Erstens können ehrenamtliche Mitarbeiter/-innen Indikatoren für Besonderheiten der bezahlten Arbeitnehmer/-innen im Nonprofit Sektor $\operatorname{sein}^{23}$. Der Arbeitsspendenhypothese zufolge, akzeptieren Arbeit-nehmer/-innen im Nonprofit Sektor niedrigere Löhne, wie weiter oben dargestellt, und „spenden“ damit gleichsam einen Teil ihres Einkommens, weil sie eine für sie sozial erwünschte Aufgabe verrichten (vgl. z.B. Hansmann 1980). Ehrenamtliche Arbeitskräfte können als der Extremfall der Arbeitsspendenhypothese gesehen werden, da sie quasi die gesamte Arbeitszeit „spenden“ (vgl. Leete 2006: 166). Sowohl das Vorhandensein als auch die Anzahl der ehrenamtlichen Mitarbeiter/-innen können daher ein Indikator für ein hohes $\mathrm{Ma}$ an sozialer Erwünschtheit der Tätigkeit bzw. der Mission der NPO und der von ihr angebotenen Stellen sein. Das Vorhandensein von ehrenamtlichen Arbeitskräften bzw. eine höhere Anzahl ehrenamtlicher Mitarbeiter/-innen würde folglich auf niedrigere Löhne der bezahlten Beschäftigten hinweisen. Dieser Effekt sollte sich besonders bei gut bezahlten Arbeitskräften (d.h. bei den oberen Lohnquantilen) zeigen, da der Arbeitsspendenhypothese zufolge Führungskräfte einer Organisation die sozialen

22 Allerdings gibt es seitens der UN Bemühungen, den Nonprofit Sektor statistisch besser zu erfassen (siehe dazu: UN 2003). Bis zum Jahr 2007 haben sich weltweit 28 Länder dazu verpflichtet, eine solche Satellitenrechnung durchzuführen (vgl. Salamon et al. 2007).

23 Bezahlte Arbeitnehmer/-innen in NPO betätigen sich überdies in ihrer Freizeit oft selbst ehrenamtlich, und zwar häufiger als Arbeitnehmer/-innen aus dem gewinnorientierten oder öffentlichen Sektor (vgl. Rotolo, Wilson 2006). 
Auswirkungen der Tätigkeit der NPO besonders gut abschätzen können (vgl. Leete 2006; Preston 1989).

Zweitens können ehrenamtliche Mitarbeiter/-innen die Produktivität oder die Arbeitskosten der bezahlten Arbeitskräfte beeinflussen. Je nachdem wie das Verhältnis der beiden Gruppen - d.h. komplementär oder als Substitut - beschrieben werden kann, können freiwillige Mitarbeiter/-innen die Notwendigkeit, Effizienzlöhne zu zahlen, verringern (vgl. Borjas 2005: 463ff.). Außerdem ist es möglich, dass durch die An-wesenheit Freiwilliger und der damit verbundenen höheren sozialen Kontrolle bezahlte Mitarbeiter/-innen weniger dazu geneigt sind, shirking zu betreiben.

Ehrenamtliche können auch die Kosten der Arbeitnehmerfluktuation reduzieren, da sie als eine Art „Puffer“ auftreten können, wenn bezahlte Arbeitskräfte die Organisation verlassen, was gleichzeitig die Notwendigkeit der Organisation, Effizienzlöhne zu zahlen, reduziert. Im Gegensatz zu Organisationen ohne freiwillige Arbeitskräfte, können bezahlte Arbeitskräfte mit ehrenamtlichen Mitarbeiter/-inne/n andere Aufgaben oder Verantwortlichkeiten haben, einerseits weil möglicher-weise Teile der Arbeit durch die Ehrenamtlichen ausgeführt wird, andererseits weil bezahlte Arbeitnehmer/-innen womöglich die unbezahlten ausbilden oder leiten. Je nach konkreter Aufgabe benötigen bezahlte Arbeitskräfte daher eine vergleichsweise höhere oder niedrige Qualifikation und/oder sie haben andere Aufgaben als Arbeitnehmer/-innen in NPO ohne Ehrenamtliche. Wenn Freiwillige eher ergänzend zu den bezahlten Mitarbeiter/-innen tätig sind, könnte dadurch der Arbeitsstress der bezahlten Arbeitnehmer/-innen verringert werden, sodass weniger kompensierende Löhne gezahlt werden müssen (vgl. Borjas 2005: 206ff.).

Das dritte Argument betrifft die Rent-Sharing Hypothese bzw. die Theorie der Eigentumsrechte (vgl. Borjas et al. 1983; Preston 1988). Aufgrund des Gewinnausschüttungsverbots haben Führungskräfte von NPO keinen gewinngetriebenen Anreiz, niedrige Löhne zu zahlen, um den Gewinn der Organisation zu vergrößern. Der Theorie der Eigentumsrechte zufolge minimieren Manager/innen aufgrund der Trennung zwischen Eigentum und Kontrolle der Organisation nicht zwingenderweise Kosten und zahlen höhere Löhne an sich und die bezahlten Arbeitskräfte, weil sie daraus Nutzen ziehen. Dadurch, dass einige Mitarbeiter/-innen nicht bezahlt werden müssen, da sie ehren-amtlich tätig sind, können vorhandene Renten in NPO in Form höherer Löhne an die (wenigen) bezahlten Mitarbeiter/-innen aufgeteilt werden. Die Möglichkeiten des RentSharings vergrößern sich dadurch. Ehrenamtliche würden folglich die erwartete Lohnhöhe positiv beeinflussen.

Viertens können ehrenamtliche Mitarbeiter/-innen möglicherweise direkten oder indirekten Einfluss auf den Lohnsetzungsprozess der bezahlten Arbeitskräfte nehmen. Einerseits können ehrenamtliche Mitarbeiter/-innen als Führungskräfte 
bei der Lohnsetzung seitens der NPO als Arbeitgeber direkten Einfluss nehmen, wobei a priori unklar bleibt, wie sich das auf die erwartete Lohnhöhe der Arbeitnehmer/-innen auswirkt. Andererseits reduzieren Freiwillige die Verhandlungsmacht von bezahlten Arbeitskräften, besonders wenn die Beziehung zwischen den Gruppen substitutionell ist. Wenn nämlich bezahlte Arbeitskräfte leicht durch unbezahlte ersetzt werden können, kann sich das negativ auf die Verhandlungsmacht der bezahlten Arbeitnehmer/-innen bei Lohnverhandlungen auswirken.

Schließlich kann als fünfter Punkt angeführt werden, dass Ehrenamtliche die Organisationsgröße erhöhen. Normalerweise wird diese über die Anzahl der Vollzeitäquivalente $^{24}$ gemessen. Inkludiert man ausschließlich bezahlte $\mathrm{Be}$ schäftigte, um die Größe einer NPO zu messen, kann die tatsächliche Organisationsgröße in vielen Fällen stark unterschätzt werden. Löhne und Organisationsgröße korrelieren, wie schon dargestellt, positiv miteinander (vgl. z.B. Lallemand et al. 2005; Lallemand et al. 2007a; Medoff, Brown 1989; Oi, Idson 1999). Die Lohnhöhe eines Arbeitnehmers bzw. einer Arbeitnehmerin einer NPO kann demnach positiv mit der Anzahl der freiwilligen Mitarbeiter/-innen korrelieren, was als Größeneffekt interpretiert werden kann.

Zusammenfassend kann festgehalten werden, dass insgesamt fünf Gründe für einen Einfluss Freiwilliger auf die Lohnhöhe von bezahlten Arbeitskräften sprechen. Dazu zählen, dass Ehrenamtliche ein Indikator für das Existieren der Arbeitsspendenhypothese sein können, dass sie die Produktivität oder die Arbeitskosten der bezahlten Arbeitnehmer/-innen beeinflussen können, dass sie die Rent-Sharing Möglichkeiten für die bezahlten Mitarbeiter/-innen erhöhen, dass sie direkt oder indirekt den Lohnverhandlungsprozess beeinflussen und dass sie die Organisationsgröße verändern.

In Folge werden Gründe präsentiert, warum Spenden und öffentliche Subventionen die Lohnhöhe beeinflussen können.

\subsection{Finanzierung der NPO}

Die Spendenbereitschaft in der österreichischen Bevölkerung kann als relativ hoch eingeschätzt werden. Umfragen zufolge, geben rund $80 \%$ der Bevölkerung an, mindestens einmal im Jahr eine Geldspende gegeben zu haben (vgl. Österreichisches Institut für Spendenwesen 2007: 6). Betrachtet man allerdings das Spendenvolumen, liegt Österreich mit einem Spendenausmaß von geschätzten 410 Millionen Euro jährlich im Vergleich zu anderen OECD Ländern

24 Im Kontext der NPO lassen sich z.B. auch die Anzahl der betreuten Kinder in Kindergärten als alternative Messgröße für Organisationsgröße finden (vgl. Mocan, Tekin 2003; Preston 1988). 
im Mittelfeld (vgl. Österreichisches Institut für Spendenwesen 2007: 8 und 14). Die gespendete Summe geht aber nicht notwendigerweise nur an NPO. In Österreich, so stellt das Österreichische Institut für Spendenwesen fest, wird „eher oft als viel gespendet" (Österreichisches Institut für Spendenwesen 2007: 8). Gespendet wird häufig für die Bereiche Gesundheit und Soziales, für Entwicklungszusammenarbeit, Umwelt und für den Tierschutz.

Ein großer Teil der Einnahmen für NPO stammt aus staatlichen Förderungen und Leistungserlösen (vgl. Schneider et al. 2007: 62). So stammen nur etwa 7$8 \%$ der Einnahmen aus Spenden, während geschätzte $13 \%$ aus Subventionen stammen und ca. 30\% Einnahmen aus Leistungsverträgen (vgl. Neumayr et al. 2007: 8). Hochgerechnet be-laufen sich die Einnahmen der österreichischen Nonprofit Organisationen im Jahr 2005 in Österreich aus dem öffentlichen Sektor auf ca. 2 Milliarden Euro (vgl. Neumayr et al. 2007: 7).

NPO produzieren häufig Güter und Dienstleistungen, die Eigenschaften von öffentlichen Gütern aufweisen (vgl. Weisbrod 1977; Weisbrod 1988). NPO, die Güter und Dienstleistungen mit Kollektivgut-eigenschaften produzieren, erhalten vermehrt finanzielle Zuwendungen in Form von Spenden und Subventionen im Vergleich zu NPO, die Güter und Dienstleistungen mit Eigenschaften eines Privatgutes produzieren (vgl. Brown, Slivinski 2006: 143). Die Abhängigkeit von externer Finanzierung durch Spenden und Subventionen kann Auswirkungen auf die Löhne haben.

Wenn eine Organisation Subventionen erhält, kann das bedeuten, dass zusätzliche Renten zur Verfügung stehen, die mittels Rent-Sharing verteilt werden können. Die Organisation hat durch die öffentliche Finanzierung womöglich ein sichereres Einkommen als NPO, die sich hauptsächlich aus unbeständigeren Finanzierungsarten wie Spenden oder Umsatzerlösen finanzieren. Daraus kann abgeleitet werden, dass Subventionen die Lohnhöhe positiv beeinflussen. Wenn der Staat als Subventionsgeber auftritt, könnte er von den NPO als „Lückenfüller" betrachtet werden, der eventuell auftretende Differenzen zwischen Einnahmen und Ausgaben begleicht, sodass die Budgetbeschränkung einer Organisation zum Teil aufgehoben wird. ${ }^{25}$ Wenn eine Organisation mit hoher Wahrscheinlichkeit damit rechnen kann, dass Ausgaben, die nicht durch Einnahmen gedeckt sind, durch den Staat beglichen werden, könnte sie insgesamt weniger dazu gezwungen sein, Rücklagen zu bilden, und kann daher höhere Löhne zahlen. Der Staat als Subventionsgeber könnte demnach als relativ sichere Einnahmequelle betrachtet werden.

25 Siehe dazu die Theorie der weichen Budgetbeschränkung (, soft budget constraint'; vgl. z.B. Kornai et al. 2003), die ursprünglich durch Kornai (vgl. z.B. 1986) für sozialistische Staaten aufgestellt wurde, später jedoch auch auf marktwirschaftliche, gewinnorientierte Unternehmen, Nonprofit Organisationen und private Haushalte ausgedehnt wurde. 
Andererseits kann dies auch bedeuten, dass (staatliche) Geldgeber möglicherweise Einfluss auf die Personalstruktur und die Löhne in der NPO selbst haben. Als Beispiel dafür kann das Feld der Langzeitpflege in Österreich genannt werden, wo zwischen den öffentlichen Geldgebern und den Anbietern der Dienstleistungen (d.h. den Pflegeheimen) fixe Patienten-Personalquoten vertraglich vereinbart werden. Zum Teil werden auch Höchstgrenzen für Personalkosten, die durch den öffentlichen Förderer refundiert werden, vereinbart. Folglich können dadurch keine höheren Löhne bezahlt werden und höhere Subventionen würden Löhne negativ beeinflussen.

Spenden sind freiwillige Zuwendungen von Privatpersonen und Firmen ${ }^{26}$. Auch hier ist a priori nicht unmittelbar klar, ob Spenden die Lohnhöhe positiv oder negativ beeinflussen. Einerseits können höhere Spenden, wie im Fall der Subventionen, für die NPO zusätzliches Einkommen darstellen, was wiederum die Rent-Sharing Möglichkeiten erhöht. Dadurch können höhere Spenden Löhne positiv beeinflussen. Andererseits unterliegen NPO einem Legitimationsdruck gegenüber Klient/-inne/n, Geldgeber/-inne/n, ehrenamtlichen Mitarbeiter/inne/n, Medien und der allgemeinen Öffentlichkeit. Hohe Löhne in diesem Segment würden als Verschwendung oder fehlende intrinsische Motivation aufgefasst werden (vgl. Oster 1998). So ist es möglich, dass Konsument/-inne/n oder Spender/-innen die Lohnhöhe der Mitarbeiter/-innen von NPO als Signal für Produktivität verstehen. Wenn die Qualität der Dienstleistungen der Organisationen nicht oder nur mit hohem Aufwand eingeschätzt werden kann, können niedrige Löhne darüber Auskunft geben, dass Spenden effektiv zur Erfüllung des Missions-zwecks eingesetzt werden (Hansmann 1980). Auch nach Preston (1988: 339) werden Spenden als eine positive Funktion von Organisationseffizienz und dem öffentlichen Nutzen, den die NPO produziert, modelliert. Hohe Effizienz und hoher öffentlicher Nutzen werden mit mehr Spenden „belohnt“. In diesem Fall würden höhere Spenden zu niedrigeren Löhnen führen, da niedrige Löhne eine Konzentration auf die Mission der Organisation signalisieren, während hohe Löhne als Verschwendung interpretiert werden (vgl. Preston 1988: 344f.). Spender/-innen interessiert, wie die NPO ihre Spende verwendet (vgl. z.B. Brown, Slivinski 2006: 143ff.). Sie spenden Geld an jene NPOs, mit deren Mission und Verhalten sie einverstanden sind. So könnten Spender/-innen auf den „Preis“ einer Spende reagieren (vgl. Brown, Slivinski 2006: 144). Der „Preis“ einer Spende gibt an, wie viel Prozent eines gespendeten Euros tatsächlich in die Bereitstellung des Guts bzw. der Dienstleistung der NPO gehen und nicht in Fundraising oder die Administration fließen. Wenn Spender/-innen in hohem Maße auf den „Preis“ der Spende reagieren, d.h. sensibel bezüglich der

26 Leider erlauben die Daten keine Trennung der Spendenvariable in private Spenden und Firmenspenden, obwohl eine getrennte Untersuchung der Effekte dieser beiden Kategorien auf die Lohnhöhe interessant wäre. 
Gemeinkosten einer Organisation reagieren, dann stehen die Organisationen unter Druck, Löhne möglichst niedrig zu halten. Tinkelman und Mankaney (2007) finden tatsächlich eine postitive Korrelation zwischen Spenden und administrativer Effizienz, besonders wenn die Organisation hauptsächlich von Spenden finanziert wird. Zusätzliche Spenden werden für die Produktion zusätzlicher Güter und Dienstleistungen verwendet.

Dem Argument widersprechend, kann angeführt werden, dass der oben angeführte negative Zusammenhang zwischen Spenden und der Lohnhöhe nur dann schlagend wird, wenn die Löhne der allgemeinen Öffentlichkeit und damit potentiellen Spender/-inne/n bekannt sind. Dies kann für Österreich nicht angenommen werden, da die Organisationen dies nicht öffentlich machen müssen.

Subventionen und Spenden können besonders die Löhne am oberen Ende der Lohnverteilung beeinflussen. Hohe Einnahmen aus Spenden und Subventionen können ein Indikator für besondere Fähigkeiten und Leistungen der Manager/innen der NPO darstellen, womit höhere Spenden und Subventionen zu höheren Löhnen am oberen Ende der Lohnverteilung führen sollten (vgl. Hallock 2002: 399f). Andererseits können Einnahmen aus Spenden und Subventionen auch insofern negativen Einfluss auf die Löhne am oberen Ende der Lohnverteilung haben, da sie hohe Kontrolle der NPO von außen durch unterschiedliche Stakeholder signalisieren und deshalb die Legitimationsproblematik besonders für Führungskräfte relevant sein kann (vgl. Hallock 2002).

Zusammenfassend können öffentliche Subventionen und Spenden einerseits Renten der NPO erhöhen, was in Folge zu höheren Löhnen führen kann, können andererseits den Druck auf die Organisation erhöhen, niedrige Löhne zu zahlen, da sie unter öffentlichem Legitimationsdruck stehen.

\section{Vorhandene empirische Evidenz}

Die relevante vorhandene empirische Evidenz im Bereich der Lohnhöhe im Nonprofit Sektor stammt hauptsächlich aus dem amerikanischen Raum. Bei einem Überblick über die existierenden Beiträge zur Lohnhöhe fällt auf, dass im Mittelpunkt des Interesses ein Vergleich der Lohnhöhe im Nonprofit Sektor mit jener im gewinnorientierten Bereich steht. Dabei beschränken sich einige Forscher/-innen bei der Analyse der Lohnhöhe auf bestimmte Branchen, andere verwenden Daten aus allen Wirtschaftsbereichen. Weiterhin fällt auf, dass die Variablen aus der Forschungsfrage der vorliegenden Arbeit - ehrenamtliche Mitarbeiter/-innen, Spenden und Subventionen - bislang kaum als Bestimmungsgrößen der Lohnhöhe in die Lohnfunktion aufgenommen wurden.

Gemeinhin wird vermutet, dass die Lohnhöhe im Nonprofit Sektor niedriger ist als jene im gewinnorientierten und im öffentlichen Sektor (siehe dazu z.B. Meszaros 2007, Warwick 2007: 12f.). In beiden eben genannten (Zeitungs-)Arti- 
keln wird davon berichtet, dass keine oder wenig Daten zu Löhnen im Nonprofit Sektor bekannt sind, gleichzeitig wird jedoch festgehalten, dass die Löhne im Nonprofit Sektor niedriger als in anderen Sektoren sind. Auch in der Stellenanzeige, die zu Beginn der Arbeit präsentiert wurde, wird vermutlich unterstellt, dass niedrigere Löhne üblich sind. Unter Forscher/-innen, die empirisch untersucht haben, ob es Unterschiede in der Lohnhöhe zwischen NPO und gewinnorientierten Unternehmen gibt, herrscht hingegen kein Konsens darüber. Unterschiede in den verwendeten Methoden, Daten oder Datenniveaus sind ein Grund für die Vielfalt an Ergebnissen.

Im Folgenden werden vorhandene Studien kurz vorgestellt. Dabei soll besonderes Augenmerk einerseits auf die Ergebnisse, andererseits auf die Vorgehensweise bei der Modellierung der Lohnfunktion und auf die inkludierten Variablen, insbesondere mit Blick auf Ehrenamtliche, Spenden und Subventionen, gerichtet werden. Zuerst werden Analysen vorgestellt, die Daten aus allen Wirtschaftsbereichen verwenden, dann wird auf Studien eingegangen, die den Lohnhöhenvergleich nur innerhalb bestimmter Wirtschaftsbereiche ziehen.

\subsection{Branchenübergreifende Analysen}

In einer der ersten Untersuchungen findet Preston (1989) für die USA mit Individualdaten aus den Jahren 1979 und 1980 einen branchenübergreifenden, niedrigeren Lohn für qualifizierte Arbeitnehmer/-innen und Führungskräfte sowie für Bürokräfte und Arbeitnehmer/-innen im Verkauf im Nonprofit Sektor nach Kontrolle von Humankapital-, Branchen-, Berufsunterschieden, sowie der Kontrolle von Unterschieden in der Arbeitsautonomie und Flexibilität (vgl. Preston 1989). Führungskräfte und qualifizierte Facharbeiter/-innen im Nonprofit Bereich verdienen der Studie zufolge um 15,2\% weniger als im gewinnorientierten Bereich, Bürokräfte und Arbeitnehmer/-innen im Verkauf um $6,1 \%$ weniger.

Shackett und Trapani (1987) verwenden US-amerikanische Individual-daten von Arbeitnehmer/-inne/n einer Längsschnittstudie aus den Jahren 1966 bis 1977. Sie untersuchen getrennt für Männer und Frauen, ob die Marktstruktur einen Einfluss auf die Lohnhöhe hat. Dabei vergleichen sie die Löhne in regulierten gewinnorientierten Firmen, NPO und öffentlichen Unternehmen mit denen unregulierter gewinnorientierter Organisationen. Als Kontrollvariable verwenden sie Humankapital-variable auf Individualebene. Für Frauen verwenden sie ein Modell der Selbstselektion, in dem die Ergebnisse um die Wahrscheinlichkeit, nicht am Arbeitsmarkt teilzunehmen, korrigiert werden (vgl. Shackett, Trapani 1987: 521f.). Es zeigt sich, dass Männer auf regulierten Märkten überdurchschnittlich gut bezahlt werden, die Lohnhöhe in NPO unterdurchschnittlich hoch ist und in öffentlichen Unternehmen kein Unterschied im Vergleich zu unregulierten Märkten festzustellen ist. Frauen werden im Vergleich zu un- 
regulierten Märkten in allen drei Kategorien, d.h. in regulierten Märkten, in NPO und im öffentlichen Bereich, überdurchschnittlich gut bezahlt. Es zeigt sich, dass Frauen in diesen Bereichen überdurchschnittlich oft Berufe, die hohe Ausbildung verlangen, innehaben. Jedoch ändert sich das Ergebnis selbst nach Kontrolle des Berufs nicht (vgl. Shackett, Trapani 1987: 529).

Leete (2001), die ebenfalls US-amerikanische Individualdaten aus dem Jahr 1990 verwendet, führt ausführliche Kontrollen von Branchen und Berufskategorien ein und stellt bei Verwendung der Kontrollvariablen keine Lohndifferenziale mehr fest. Desweiteren kontrolliert sie für Humankapital, Regionen und Arbeitszeit. Obwohl sie keine übergreifenden siginifikanten Lohnunterschiede zwischen dem Nonprofit Sektor und dem gewinnorientierten Bereich findet, zeigt sie Differenzen für einzelne Berufsgruppen auf. Zwischen Manager/-inne/n, Fachkräften, technischen Angestellten, Angestellten im Handel und Verwaltungsangestellten im Nonprofit Sektor und dem gewinnorientierten Sektor findet sie keine Differenzen in der Lohnhöhe. Sehr wohl liegen aber Lohndifferenzen bei Beschäftigten im Dienstleistungs-bereich vor, wobei jene im Nonprofit Sektor höhere Löhne aufwiesen, und bei handwerklichen Berufen, bei denen die Löhne im Nonprofit Sektor niedriger waren als im gewinnorientierten Bereich (vgl. Leete 2001: 153). Beim Vergleich der Branchen, in denen die Beschäftigung im Nonprofit Bereich mindestens 5\% beträgt, hat ca. die Hälfte aller Branchen ein positives, die andere Hälfte ein negatives Nonprofit Lohndifferenzial. Höhere Löhne werden in NPO im Bereich der Pflege, des Hochschul-, Versicherungs- und Krankenanstaltenwesens sowie im Bereich der Kinderbetreuung bezahlt. Geringere Löhne hingegen im Bereich der Kunst und Kultur, in Schulen und im Bereich der Weiterbildung bzw. Umschulung (Leete 2001: 158f.).

Ruhm und Borkoski (2003) kommen in ihrer Studie, in der sie US-amerikanische Paneldaten aus den Jahren 1994 bis 1998 mit Arbeitnehmer/-innen aus allen Branchen verwenden, zum Schluss, dass Personen im Nonprofit Sektor durchschnittlich $3 \%$ weniger verdienen als entsprechende Personen im gewinnorientierten Sektor mit ähnlichen Attributen. Arbeitsplätze im Nonprofit Bereich sind vorwiegend in Branchen mit niedrigen Löhnen zu finden. Den niedrigeren Lohn erklären sie aber durch die Arbeitsbedingungen, die im Nonprofit Bereich besser sein sollen, Individuen im Nonprofit Sektor arbeiteten oft weniger Stunden. Außerdem sind diese Arbeitsplätze möglicherweise wegen guter Arbeitsbedingungen begehrte Arbeitsplätze, was in Folge durch ein höheres Arbeitsangebot zu niedrigeren Löhnen führt. Nach Kontrolle von Arbeitsplatz- und Arbeitnehmer/-innencharakteristika stellen sie jedoch keine Unterschiede in der Bezahlung der beiden Gruppen mehr fest. Wenn allerdings ausschließlich jene Branchen miteinander verglichen werden, in denen NPO vorwiegend aktiv sind, ist ein Lohnzuschlag im Nonprofit Sektor feststellbar. Im Nonprofit Sektor ist ihrer Meinung nach Wettbewerb zur Bestimmung der 
Löhne eine äußerst relevante Komponente (vgl. Ruhm, Borkoski 2003: 993). Durch die Verwendung von Paneldaten ist es außerdem möglich, die Lohnänderung zu beobachten, wenn ein/e Arbeitnehmer/-in vom gewinnorientierten in den nicht-gewinnorientierten Sektor wechselt und umgekehrt. Auch hier werden keine nennenswerten Unterschiede zwischen Personen, die vom gewinnorientierten Sektor in den Nonprofit Sektor wechseln und denen, die in die umgekehrte Richtung wechseln, festgestellt (vgl. Ruhm, Borkoski 2003: 1012f.).

Die Unterschiede in den Ergebnissen der genannten Studien sind auch dadurch $\mathrm{zu}$ erlären, dass sehr unterschiedliche Abstufungen in der Kontrolle nach Branche und Berufsgruppe getroffen wurden. Während Preston (1989) nicht nach Branchen kontrolliert, führt Leete (2001) 206 Branchen und 226 Berufsgruppenkategorien ein. Ruhm und Borkoski (2003) verwenden wiederum sechs Branchen und sieben Berufs-unterteilungen. Solange in den Berechnungen nicht dafür kontrolliert wird, weisen die Studien niedrigere Löhne für den Nonprofit Sektor aus. Sobald jedoch Branchendummys in die Modelle integriert werden, tendieren die Lohndifferenziale gegen null (vgl. Leete 2006: 163). Daraus lässt sich schließen, dass NPO hauptsächlich in Niedriglohn-branchen agieren. Es lässt sich weiters festhalten, dass in den branchenweiten Analysen weder ehrenamtliche Mitarbeiter/-innen noch Spenden und Subventionen als kontrollierende Variable verwendet wurden.

\subsection{Branchenanalysen}

Studien, die einzelne Branchen untersuchen, finden sich vorwiegend im Gesundheits-, Pflege- und Kinderbetreuungsbereich, da dies traditionell jene Branchen sind, in denen sowohl öffentliche, gewinnorientierte als auch nicht gewinnorientierte Anbieter am Markt agieren. Am Rande sei erwähnt, dass zwei Beiträge zu Unterschieden der Lohnhöhe zwischen Rechtsanwälten im Nonprofit und im Forprofit Sektor existieren (vgl. Weisbrod 1983; Goddeeris 1988). Hier wird festgestellt, dass Anwälte im Nonprofit Sektor um ca. 20\% weniger verdienen (vgl. Weisbrod 1983), sobald jedoch für Selbstselektion kontrolliert wird, verschwindet dieser Unterschied (vgl. Goddeeris 1988).

\subsubsection{Altenheime}

Für den Altenheimsektor finden Borjas, Frech und Ginsburg (1983) mit Daten aus den Jahren 1973 und 1974 für die USA höhere Löhne im öffentlichen Sektor und im Nonprofit Sektor im Vergleich zum gewinnorientierten Sektor, wobei sie nach Unterschieden im Human-kapital und den Regionen kontrollieren. Eine Ausnahme bilden Mitarbeiter/-innen mit kirchlicher Zugehörigkeit in kirchennahen NPO, die weniger verdienen als ihre Kolleg/-inn/en im gewinnorientierten Bereich (vgl. Borjas et al. 1983: 238). Die Autoren untersuchen auch, ob Löhne auf Änderungen in der Finanzierungsstruktur der Altenheime 
reagieren. Es zeigt sich, dass sich die Lohnhöhe im Nonprofit Sektor weniger stark ändert, wenn sich die öffentliche Finanzierung ändert, als bei gewinnorientierten Unternehmen (vgl. Borjas et al. 1983: 239). Eine Änderung der Finanzierung von Einzelabgeltung auf pauschale Abgeltung hat demnach in gewinnorientierten Unternehmen größere Auswirkungen auf Löhne als im Nonprofit Sektor.

Holtmann und Idson (1993) finden mit US-amerikanischen Daten aus 1985 ein positives Lohndifferenzial für Nonprofit Krankenschwestern bzw. Krankenpfleger in Alten- und Pflegeheimen, das allerdings auf der höheren Ausbildung der Individuen im Nonprofit Bereich beruht. Sie legen dieses Differenzial derart aus, dass Nonprofit Heime Dienstleistungen höherer Qualität anbieten bzw. produzieren und infolge dessen eine höhere Nachfrage nach besser qualifizierten Kranken-pfleger/-inne/n haben, was auch zu höheren Löhnen führt. Sie kontrollieren in der Analyse nach Unterschieden im Humankapital und der Organisationsgröße. Das noch verbleibende Lohndifferenzial wird von den Autoren durch unbeobachtbare Qualitätsunterschiede der Arbeitnehmer/-innen erklärt.

In einer ähnlichen Vorgehensweise führten Noguchi und Shimizutani (2007) einen Lohnvergleich zwischen Nonprofit und gewinnorientierten Krankenpfleger/-inne/n und Heimhelfer/-inne/n in Japan durch. Dazu verwenden sie japanische LEE-Daten aus dem Jahr 2000. Neben Humankapital, Organisations- und Angebotscharakteristika und einem Regionendummy nehmen sie auch nicht-monetäre Lohnnebenleistungen als unabhängige Variablen in die Lohnfunktion auf. Unterschiedliche Produktcharakteristika und nicht-monetäre Nebenleistungen sollen Unterschiede in der Produktqualität und den Arbeitsannehmlichkeiten berücksichtigen. Sie finden höhere Löhne im Nonprofit Sektor, außerdem zeigen sie, dass in diesem Sektor Alter und Erfahrung zu einer größeren Lohnsteigerung führt als im gewinnorientierten Bereich. Die höheren Löhne im Nonprofit Sektor führen sie auf die Rent-Sharing Hypothese zurück, da ihrer Meinung nach NPO keine bessere Produktqualität anbieten (vgl. Noguchi, Shimizutani 2007: 13).

Mosca, Musella und Pastore (2007) vergleichen mit italienischen Daten aus dem Jahr 1998 Löhne im Bereich Pflege, Rehabilitation und persönlicher Assistenz. Sie finden durchgehend niedrigere Löhne im Nonprofit Sektor, wobei nach Unterschieden im Humankapital, in der Arbeitszufriedenheit, in den Arbeitsbedingungen und im Beruf kontrolliert wird. Sie zeigen außerdem, dass im Nonprofit Sektor mehr Individuen teilzeitbeschäftigt sind. Das Lohndifferenzial erklären sie mit der höheren Arbeitszufriedenheit der Beschäftigten im Nonprofit Sektor. Niedrige Löhne im Nonprofit Sektor können dieser Studie zufolge mit der Arbeitsspendenhypothese interpretiert werden, dass also die Arbeitnehmer/innen im Nonprofit Sektor vermehrt bereit sind, einen Teil ihres Lohn zu ,spenden“. 


\subsubsection{Kinderbetreuung}

Im Bereich der Kinderbetreuung gibt es einige sehr aufschlussreiche Studien, die die Lohnhöhe von Kindergärtner/-inne/n im Nonprofit und Forprofit Bereich vergleichen. Für amerikanische Kindergärten findet Preston (1988: 348) mit älteren Organisationsdaten aus dem Jahre 1976/77, dass Arbeitnehmer/-innen im Nonprofit Bereich um fünf bis zehn Prozent mehr verdienen als jene im gewinnorientierten Bereich. Dies gilt allerdings nur für den öffentlich subventionierten und regulierten Markt. Im kompetitiveren, nicht regulierten Markt wird kein signifikantes Differenzial zwischen den beiden Sektoren festgestellt. Da für die Berechnungen nur Organisationsdaten zur Verfügung stehen, verwendet die Autorin als Kontrollvariablen aggregiertes Humankapital auf Organisationsebene, sowie Organisationscharakteristika, Regionendummys und einen Nonprofit Dummy (vgl. Preston 1988: 343). Besonders interessant in diesem Modell ist die Aufnahme der Höhe der "Spenden“ an die Einrichtung. Die Autorin definiert „Spenden“ als Einnahmen, die nicht vom Empfänger bzw. der Empfängerin der Dienstleistung kommen, womit darunter auch Subventionen fallen (vgl. Preston 1988: 344). Sie findet, dass höhere "Spenden“" zu höheren Löhnen führen, wenngleich der Effekt nur sehr klein ist (Preston 1988: 348).

Mocan und Viola (1997) untersuchen mithilfe eines detailreichen LEE-Datensatzes aus dem Jahr 1993 Kinderbetreuungsstätten in vier amerikanischen Bundesstaaten. Dieser Datensatz ist nicht nur deshalb besonders beachtenswert, weil er Arbeitnehmer- und Arbeitgeberinformationen beinhaltet, sondern auch, weil er detaillierte Angaben zur Qualität der Dienstleistung, die aufgrund von teilnehmender Beobachtung aufgezeichnet wurden, beinhaltet. Untersucht wird das Lohndifferenzial von Pädagog/-inn/en und Hilfskräften zwischen NPO und gewinnorientierten Einrichtungen. Öffentliche Kinderbetreuungseinrichtungen werden in dieser Analyse zum Nonprofit Sektor gezählt (vgl. Mocan, Viola 1997: 36). Neben Humankapitalvariablen, gingen auch Organisationscharakteristika, Angebotscharakteristika, Regionendummys, die regionale Arbeitslosenrate, ein alternativer Lohn im Servicesektor und ein Maß zur Messung von unbeobachteter Produktivität als unabhängige Variablen in die Schätzgleichung ein. Ein übergreifendes Lohndifferenzial zwischen den beiden Sektoren wird in der Untersuchung nicht festgestellt. Jedoch gibt es Unterschiede in der Bezahlung innerhalb des Nonprofit Sektors je nach Eigentümer/-innen. Kirchennahe NPO weisen geringere Löhne auf, öffentliche Kindergärten, die in dieser Untersuchung zum Nonprofit Sektor zählen, oder Einrichtungen mit hohem Anteil an öffentlicher Unterstützung zahlen höhere Löhne für Pädagog/-inn/en, nicht aber für Helfer/-innen. Es wurde auch ein Zusammenhang mit der ethnischen Zusammensetzung der Belegschaft hergestellt. Kindergärten mit hoher öffentlicher Unterstützung zahlen Minderheiten vergleichsweise höhere Löhne, kirchennahe zahlen Minderheiten weniger. Angestellte, die Gewerkschaftsmitglieder sind, weisen höhere Löhne auf. Hohe Alternativlöhne führen er- 
wartungsgemäß zu höheren Löhnen, hohe Arbeitslosigkeit in der Region schmälert die Löhne von Hilfskräften, steigert aber die Löhne von Pädagog/inn/en. Höhere Gewinne sind positiv mit den Löhnen von Hilfskräften korreliert, haben aber keinen Zusammenhang mit denen der Pädagog/-inn/en. Die Löhne der letztgenannten Guppe steigen auch mit der Kindergartengröße, nicht aber die der Helfer/-innen. Erklärt wird diese Tatsache damit, dass wegen sinkender Überwachungsmöglichkeiten den Pädagog/-inn/en Effizienzlöhne gezahlt werden, da aber diese die Helfer/-inn/en auch bei wachsender Kindergartengröße überwachen können, spielen für Hilfskräfte Effizienzlöhne keine Rolle (vgl. Mocan, Viola 1997: 34).

Eine neuere Untersuchung aus dem Jahr 2003 von Mocan und Tekin, die denselben LEE-Datensatz verwenden, weist durch eine unterschiedliche methodische Vorgehensweise für den Nonprofit Sektor höhere Löhne aus (vgl. Mocan, Tekin 2003). Im Modell wird zwischen Vollzeit- und Teilzeitkräften im Nonprofit Sektor und im gewinnorientierten Sektor unterschieden, denen unterschiedliche Lohnfunktionen zugrunde liegen. Der Grund für die Trennung in verschiedene Lohnfunktionen je nach Sektor ist, dass Arbeitnehmer/-innen im Nonprofit Sektor systemtisch anders sein könnten als jene im gewinnorientierten Bereich. Deshalb ist eine einfache Sektorendummyvariable nach Ansicht der Autor/-inn/en nicht ausreichend, da sie möglicherweise endogen ist und die Schätzer durch unbeobachtete Arbeitnehmercharakteristika verzerrt sind (vgl. Mocan, Tekin 2003: 39). In die Berechnungen gehen ähnlich wie oben Humankapitalvariablen, Organisationscharakteristika, Angebotscharakteristika, Regionendummys, die lokale Arbeitslosenrate und die Frage, ob die Einrichtung Teil einer Kette ist als unabhängige Variable in die Schätzgleichung ein. Hier wird außerdem die Tatsache zunutze gemacht, dass der Datensatz eine Frage beinhaltet, die einen Test der Arbeitsspendenhypothese ermöglicht. Dabei werden die Arbeitnehmer/-innen gefragt, ob sie glauben, eine wichtige Tätigkeit zu verrichten, die getan werden muss. Diejenigen, die dieser Aussage zustimmten, verdienten um zwei bis fünf Prozent weniger, nach Kontrolle von anderen Einflussfaktoren (vgl. Mocan, Tekin 2003: 45). Dieser Effekt wird jedoch ausschließlich in NPO gemessen, was mit der Arbeitsspendenhypothese erklärt wird. In gewinnorientierten Einrichtungen gibt es hier einen positiven Zusammenhang bei Vollzeitkräften, was damit erklärt wird, dass diese Einstellung zur eigenen Arbeit zu höherer Produktivität führt, was im gewinnorientierten Bereich zu höheren Löhnen führt. Minderheiten selbstselektieren in NPO. Einrichtungen, die Teil einer Kette sind, weisen niedrigere Löhne auf, Gewerkschaftsmitglieder höhere. Während große gewinnorientierte Unternehmen höhere Löhne zahlen, ist interessanterweise der Zusammenhang zwischen Organisationsgröße und Lohn in NPO negativ (vgl. Mocan, Tekin 2003: 45f.). 
In beiden Studien wurde eine Variable in die Lohngleichung eingefügt, die angibt, ob die Organisation öffentliche finanzielle Unterstützung, d.h. Subventionen, erhält. Während Mocan und Tekin (2003: 44) finden, dass öffentliche Unterstützung bei Vollzeitkräften zu niedrigeren Löhnen führt und zu höheren bei Teilzeitkräften, finden Mocan und Viola (1997: 42) keinen statistisch signifikanten Einfluss.

\subsection{Zusammenfassung}

Die auf den nächsten Seiten folgenden Tabellen geben nochmals einen vergleichenden Überblick über vorhandene Studien.

Zusammenfassend lässt sich festhalten, dass keine bislang erfolgte Analyse zur Lohnhöhe im Nonprofit Sektor ehrenamtliche Mitarbeiter/-innen als Erklärungsfaktor heranzieht. Die Befunde der Auswirkungen von Subventionen auf die Lohnhöhe von bezahlten Arbeitnehmer/-innen im Nonprofit Sektor sind gemischt (vgl. hierzu Borjas et al. 1983, Mocan, Viola 1997 und Mocan, Tekin 2003). Für Spenden im weiteren Sinne findet man einen positiven Einfluss auf die Lohnhöhe (vgl. Preston 1988). Es wurden Analysen dargestellt, die die Lohnhöhe zwischen öffentlichen, gewinnorientierten und Nonprofit Organisationen vergleichen, eine Studie, die sich explizit mit unterschiedlichen Einflussfaktoren der Lohnhöhe innerhalb des Nonprofit Sektors befasst, d.h. Unterschiede der Lohnhöhe zwischen NPO untersucht, existiert meines Wissens nach nicht. Es zeigt sich, dass ältere Studien entweder Individual- oder Organisationsdaten verwenden, neuere LEE-Daten, die sich dann aber nur auf einzelne Branchen beschränken.

Insgesamt fällt auf, dass in den vorhandenen Beiträgen Unterschiede in der Lohnhöhe zwischen öffentlichen, gewinnorientierten und Nonprofit Organisationen nicht mit einer Theorie erklärt werden bzw. nicht erklärt werden können, sondern unterschiedliche Erklärungsansätze herangezogen werden.

Die nun folgende empirische Untersuchung versucht, Lohnunterschiede zwischen Nonprofit Organisationen zu erklären, wobei besonders auf die Erklärungsfaktoren Ehrenamt, Spenden und Subventionen eingegangen wird. Dafür werden LEE-Daten für verschiedene nonprofit-relevante Branchen verwendet. 


\begin{tabular}{|c|c|c|c|c|c|c|c|c|}
\hline $\begin{array}{l}\text { Autor/ } \\
\text {-inn/en }\end{array}$ & Jahr & Daten & Datentyp & $\begin{array}{c}\text { Daten } \\
\text { aus }\end{array}$ & Modellvariablen & Hauptergebnisse & \begin{tabular}{c|} 
NP/FP \\
Lohndifferent.
\end{tabular} & Besonderes \\
\hline \multicolumn{9}{|c|}{ Branchenübergreifend } \\
\hline Preston & 1989 & USA & $\begin{array}{c}\text { Individual- } \\
\text { daten }\end{array}$ & $1979 / 80$ & $\begin{array}{l}\text { Humankapital, Branche, } \\
\text { Beruf, Regionen, } \\
\text { Arbeitsautonomie }\end{array}$ & $\begin{array}{c}\text { Manager und Fachkräfte } \\
\text { verdienen im NPS 15\% } \\
\text { weniger, Büro/Handel } 6 \% \\
\text { weniger } \\
\end{array}$ & - & \\
\hline $\begin{array}{l}\text { Shackett/ } \\
\text { Trapani }\end{array}$ & 1987 & USA & $\begin{array}{c}\text { Individual- } \\
\text { daten, } \\
\text { Panel }\end{array}$ & $\begin{array}{c}1966- \\
77\end{array}$ & Humankapital, Beruf & $\begin{array}{c}\text { Männer im NPS } \\
\text { niedrigere Löhne, Frauen } \\
\text { höhere }\end{array}$ & $-1+$ & $\begin{array}{l}\text { Löhne nach } \\
\text { Geschlechtern } \\
\text { getrennt } \\
\text { untersucht }\end{array}$ \\
\hline Leete & 2001 & USA & $\begin{array}{c}\text { Individual- } \\
\text { daten }\end{array}$ & 1990 & $\begin{array}{c}\text { Humankapital, } \\
\text { Regionendummy, } \\
\text { Arbeitszeit, Branchen, Beruf }\end{array}$ & $\begin{array}{c}\text { generell kein Unterschied, } \\
\text { einzelne Berufe positives } \\
\text { Lohndifferential }\end{array}$ & $0 /+$ & $\begin{array}{l}\text { genaue } \\
\text { Branchen- und } \\
\text { Berufsunter- } \\
\text { gliederung }\end{array}$ \\
\hline $\begin{array}{l}\text { Ruhm/ } \\
\text { Borkoski }\end{array}$ & 2003 & $\begin{array}{l}\text { USA, } \\
\text { Individuen }\end{array}$ & $\begin{array}{c}\text { Individual- } \\
\text { daten, } \\
\text { Panel }\end{array}$ & $\begin{array}{c}1994- \\
98\end{array}$ & $\begin{array}{c}\text { Humankapital, } \\
\text { Arbeitsplatzcharakteristika, } \\
\text { Branche, Beruf, } \\
\text { Arbeitsbedingungen }\end{array}$ & $\begin{array}{c}\text { kein generelles } \\
\text { Lohndifferential, } \\
\text { geringere Löhne im NP } \\
\text { Sektor beruhen auf } \\
\text { besseren } \\
\text { Arbeits(platz)bedingungen }\end{array}$ & 0 & $\begin{array}{l}\text { Lohnänderungen } \\
\text { bei AN- } \\
\text { mobilität } \\
\text { zwischen FP } \\
\text { und NP }\end{array}$ \\
\hline \multicolumn{9}{|c|}{ Alten- und Pflegeheime } \\
\hline $\begin{array}{l}\text { Borjas/ } \\
\text { Frech/ } \\
\text { Ginsburg }\end{array}$ & 1983 & $\begin{array}{c}\text { USA, } \\
\text { Kranken- } \\
\text { schwestern }\end{array}$ & $\begin{array}{l}\text { LEE } \\
\text { Daten }\end{array}$ & $1973 / 74$ & $\begin{array}{l}\text { Organisationscharakteristika, } \\
\text { Humankapital, Regionen }\end{array}$ & $\begin{array}{c}\text { Löhne im öffentl. Bereich } \\
\text { und im NPS höher als im } \\
\text { FPS, jedoch haben } \\
\text { kirchennahe AN geringere } \\
\text { Löhne } \\
\end{array}$ & + & $\begin{array}{l}\text { Finanzierungs- } \\
\text { struktur der } \\
\text { Heime }\end{array}$ \\
\hline $\begin{array}{l}\text { Holt- } \\
\text { mann/ } \\
\text { Idson }\end{array}$ & 1993 & $\begin{array}{c}\text { USA, } \\
\text { Kranken- } \\
\text { schwestern }\end{array}$ & $\begin{array}{l}\text { LEE } \\
\text { Daten }\end{array}$ & 1985 & $\begin{array}{l}\text { Organisationsgröße, } \\
\text { Humankapital }\end{array}$ & $\begin{array}{l}\text { höher Qualifizierte in } \\
\text { NPO, Ausbildung fuhrt in } \\
\text { NPO zu höheren Löhnen }\end{array}$ & + & $\begin{array}{l}\text { Qualität d. } \\
\text { Dienstleistung }\end{array}$ \\
\hline
\end{tabular}


Alten- und Pflegeheime (Fortsetzung)

\begin{tabular}{|c|c|c|c|c|c|c|c|c|}
\hline $\begin{array}{l}\text { Noguchi/ } \\
\text { Shimi- } \\
\text { zutani }\end{array}$ & 2005 & $\begin{array}{l}\text { Japan, } \\
\text { Kranken- } \\
\text { pfleger, } \\
\text { Heimhilfen }\end{array}$ & LEE Daten & 2000 & $\begin{array}{c}\text { Humankapital, } \\
\text { Organisationscharakteristika, } \\
\text { Angebotscharakteristika, nicht- } \\
\text { monetăre Zusatzleistungen, } \\
\text { Region } \\
\end{array}$ & $\begin{array}{c}\text { Alter und Erfahrung führen } \\
\text { besonders bei NPO zu höheren } \\
\text { Löhnen, höhere Lobne in NPO } \\
\text { durch Rent Sharing }\end{array}$ & + & $\begin{array}{l}\text { nicht- } \\
\text { monetäre } \\
\text { Zusatz- } \\
\text { leistungen }\end{array}$ \\
\hline $\begin{array}{l}\text { Mosca/ } \\
\text { Musella/ } \\
\text { Pastore }\end{array}$ & 2007 & $\begin{array}{l}\text { Italien, } \\
\text { Pflege, Re- } \\
\text { habilitation, } \\
\text { persönl. } \\
\text { Assistenz }\end{array}$ & $\begin{array}{l}\text { Individual- } \\
\text { daten }\end{array}$ & 1998 & $\begin{array}{c}\text { Humankapital, } \\
\text { Arbeitszufriedenheit, Region, } \\
\text { Beruf }\end{array}$ & $\begin{array}{l}\text { niedrigere Löhne im NPS, mehr } \\
\text { Teilzeitkrăfte, höhere } \\
\text { Arbeitszufriedenheit }\end{array}$ & - & $\begin{array}{l}\text { Motivation d. } \\
\text { AN, Arbeits- } \\
\text { zufriedenheit }\end{array}$ \\
\hline \multicolumn{9}{|c|}{ Kinderbetreuungsstătten } \\
\hline Preston & 1988 & $\begin{array}{l}\text { USA, } \\
3167 \\
\text { Organisa- } \\
\text { tionen }\end{array}$ & $\begin{array}{l}\text { Organi- } \\
\text { sationsdaten, } \\
\text { Querschnitt }\end{array}$ & $1976 / 77$ & $\begin{array}{l}\text { aggregiertes Humankapital auf } \\
\text { Organisationsebene, } \\
\text { Organisationscharakteristika, } \\
\text { Spendenausmaß, } \\
\text { Regionendummy, NP-Dummy }\end{array}$ & $\begin{array}{c}5-10 \% \text { mehr im NPS, Spenden } \\
\text { erhöhen NP Löhne (geringfugig), } \\
\text { in weniger kompetitiven Märkten } \\
\text { höhere NP Löhne, in } \\
\text { kompetitiven keine Lohndifferenz }\end{array}$ & + & $\begin{array}{c}\text { Unter- } \\
\text { scheidung in } \\
\text { kompetitive } \\
\text { und weniger } \\
\text { kompetitive } \\
\text { Märkte }\end{array}$ \\
\hline $\begin{array}{l}\text { Mocan/ } \\
\text { Viola }\end{array}$ & 1997 & $\begin{array}{l}4 \text { US } \\
\text { Bundes- } \\
\text { staaten, } 398 \\
\text { Organisa- } \\
\text { tionen }\end{array}$ & LEE Daten & 1993 & $\begin{array}{l}\text { Organisationscharakteristika, } \\
\text { Angebotscharakteristika, } \\
\text { Humankapital, Region, } \\
\text { Produktivitat, Produktqualităt, } \\
\text { regionale Arbeitslosenquote, } \\
\text { regionaler Alternativlohn im } \\
\text { Servicesektor }\end{array}$ & $\begin{array}{c}\text { offentliche od. NPOs mit } \\
\text { offentlicher Finanzierung: höhere } \\
\text { Löhne, kirchennahe NPOs } \\
\text { niedrigere Löhne }\end{array}$ & 0 & $\begin{array}{l}\text { Produktivităt } \\
\text { und Produkt- } \\
\text { qualităt } \\
\text { mitein- } \\
\text { bezogen }\end{array}$ \\
\hline $\begin{array}{c}\text { Mocan/ } \\
\text { Tekin }\end{array}$ & 2003 & $\begin{array}{l}4 \text { US } \\
\text { Bundes- } \\
\text { staaten, } 398 \\
\text { Organisa- } \\
\text { tionen }\end{array}$ & LEE Daten & 1993 & $\begin{array}{l}\text { Organisationscharakteristika, } \\
\text { Angebotscharakteristika, } \\
\text { Arbeitszeit, Humankapital, } \\
\text { Region, regionale } \\
\text { Arbeitslosenquote, Teil einer } \\
\text { Kette, Arbeitsspende, } \\
\text { Gewerkschaft }\end{array}$ & $\begin{array}{l}\text { "wichtiger Job" fuhrt in NPO zu } \\
\text { geringeren Löhnen, in FPO zu } \\
\text { hoberen }\end{array}$ & + & $\begin{array}{l}\text { Test der } \\
\text { Arbeits- } \\
\text { spenden- } \\
\text { hypothese }\end{array}$ \\
\hline
\end{tabular}


Abgeleitet von den in Kapitel 3 beschriebenen theoretischen Ansätzen und unter Einbezug der in Kapitel 4 thematisierten Besonderheiten von NPO wird in diesem Kapitel eine Lohnfunktion für Arbeitnehmer/-innen im Nonprofit Sektor aufgestellt. Außerdem wird auf relevante Variable näher eingegangen und die empirische Lohnfunktion abgeleitet. Schließlich werden Hypothesen zu den erwarteten Vorzeichen der Koeffizienten bei der Schätzung diskutiert.

\subsection{Die Lohnfunktion von Arbeitnehmer/-innen im Nonprofit Sektor}

Der Lohn eines Arbeitnehmers bzw. einer Arbeitnehmerin bestimmt sich aus dem Schnittpunkt von Arbeitsangebot und Arbeitsnachfrage. Die Darstellung der Herleitung der Lohnfunktion folgt in weiten Zügen Leete (2001: 140ff.), wird jedoch zum Teil aus inhaltlichen Überlegungen angepasst.

Ein Modell des Arbeitsangebots beinhaltet für gewöhnlich die Annahme, dass Arbeitnehmer/-innen Nutzen durch ihren Lohn und durch nicht-monetäre Arbeitscharakteristika erlangen. Die Tatsache, dass sie für eine NPO arbeiten und, wie oben beschrieben, ein spezielles Gut produzieren, kann beispielsweise dem Individuum einen nicht-monetären Nutzen bereiten (vgl. z.B. Leete 2001, Mosca et al. 2007).

Der Nutzen eines Arbeitnehmers bzw. einer Arbeitnehmerin $i$ in der Organisation $j$ wird folgendermaßen bestimmt:

$$
U_{i j}=U\left(W_{i j}, I W_{i j}, S A C_{j}, B A C_{j}\right)
$$

wobei $U_{i j}$ den Nutzen eines Arbeitnehmers bzw. einer Arbeitnehmerin $i$ in der Organisation $j$ darstellt, der eine Funktion des Lohns $W_{i j}$, des intrinsischen Werts der Arbeit $I W_{i j}$, der sich aus das Tatsache ergibt, dass das Individuum in einer NPO arbeitet, der sektorenspezifischen Arbeitsplatzcharakteristika $S A C_{j}$ und der berufs- bzw. stellenspezifischer Arbeitsplatzcharakteristika $B A C_{j}$ ist. Die Funktion $U$ steigt mit zunehmendem Lohn $W_{i j}$ und zunehmendem intrinsischen Wert $l W_{i j}{ }^{27}$, kann jedoch in Bezug auf sektorenspezifische Arbeitsplatzcharakteristika $S A C_{j}$ und berufsspezifische Arbeitsplatzcharakteristika $B A C_{j}$ steigen oder fallen.

Das Arbeitsangebot eines Individuums $i$ in der Organisation $j$, das den Nutzen maximiert, ergibt sich folgendermaßen (vgl. Leete 2001: 141):

27 Nach der "motivation-crowding Hypothese“ (Grepperud, Pedersen 2006; James Jr. 2005) könnten auch $W_{i j}$ und $I W_{i j}$ zusammenhängen. So könnte eventuell ein höherer Lohn die intrinsische Motivation schmälern und in Folge den intrinsischen Wert der Arbeit schmälern. 
Die Arbeitsnachfrage kann folgendermaßen beschrieben werden (Leete 2001: 141):

(3) $A N_{i j}=g\left(W_{i j}, H K_{i j}, R_{j}, D_{j}, R S_{j}\right)$

wobei $A N_{i j}$ die nachgefragte Anzahl nach Arbeitnehmer/-inne/n mit den Charakteristika eines Arbeitnehmers bzw. einer Arbeitnehmerin $i$ in der $j-t e n$ Organisation ist. $W_{i j}$, stellt wiederum den Lohn eines Arbeitnehmers bzw. einer Arbeitnehmerin $i$ in der $j$-ten Organisation dar, $H K_{i j}$ das Humankapital des $i-t e n$ Arbeitnehmers oder der $i$-ten Arbeitnehmerin, $R_{j}$ die regionalen Bedingungen, an denen der Arbeitsplatz liegt, $D_{j}$ stellt Diskriminierung dar, die aufgrund von Charakteristika, die nicht mit der Produktivität eines Individuums zusammenhängen, getroffen wird, $R S_{j}$ gibt schließlich die Bedingungen wieder, die die Verteilung von Renten innerhalb einer Organisation $j$ beeinflussen (siehe dazu unten). Die Arbeitsnachfrage hängt negativ vom Lohn ab und positiv vom Humankapital. Sie kann in Bezug auf die regionalen Bedingungen, der Diskriminierung durch die Unternehmen und den Bedingungen, die RentSharing beeinflussen, steigen oder fallen.

Der Lohn ergibt sich aus dem Schnittpunkt der Arbeitsangebots- und Arbeitsnachfragefunktion:

$$
W_{i j}=h\left(I W_{i j}, S A C_{j}, B A C_{j}, H K_{i}, R_{j}, D_{j}, R S_{j}\right)
$$

Als log-lineare Funktion wird kann sie folgendermaßen formuliert werden:

$$
W_{i j}=j+k I W_{i j}+l S A C_{j}+m B A C_{j}+n H K_{i}+o R_{j}+p D_{j}+q R S_{j}
$$

Die Bedingungen, die Rent-Sharing innerhalb einer Organisation $j$ beeinflussen, werden nun genauer spezifiziert. Bei genauerer Überlegung ist festzustellen, dass Rent-Sharing aus zwei Komponenten besteht, erstens der Höhe und zweitens der Verteilung vorhandener Renten.

Renten können logischerweise nur dann verteilt werden, wenn auch welche vorhanden sind. Organisationen müssen die Fähigkeit oder Möglichkeit besitzen, Renten zu akkumulieren. Die Höhe der Renten hängt vom Branchenwettbewerb $B W_{j}$, der Höhe finanzieller Zuwendungen $F Z_{j}$, die nicht durch den Verkauf von Waren oder Dienstleistungen entstehen, der Organisationseffizienz $O E_{\text {, }}$, der Organisationsgröße $O G_{j}$ und einem fehlenden Gewinnmaximierungsziel ${ }^{28} \mathrm{der}$ NPO $M Z_{j}$ ab. In Branchen mit hohem Wettbewerb sind die Renten kleiner. Renten wachsen mit höherer Organisationseffizienz. Die Organisationsgröße ist in-

28 Gewinnmaximierung ist NPO nicht verboten, lediglich Gewinnausschüttung. Es ist also der Spezialfall möglich, dass die NPO Gewinne maximiert, die meisten NPO maximieren wahrscheinlich aber Anderes, wie Outputmenge oder Qualität. 
sofern für die Bestimmung der Höhe der Renten relevant, als mit der Größe Skalenerträge steigen können, die wiederum Renten erhöhen. Das fehlende Gewinnmaximierungsziel von NPO wirkt sich negativ auf die Höhe der Renten aus.

Im Vergleich zur Darstellung von Leete (2001) wurde an dieser Stelle das Modell um die Komponenten finanzielle Zuwendungen und Maximierungsziel erweitert.

Zweitens muss untersucht werden, welcher Teil der vorhandenen Renten an Arbeitnehmer/-innen mittels Lohnzahlungen ausgeschüttet wird. Ob und an wen vorhandene Renten verteilt werden, hängt von den Bedingungen $a b$, die zu einer möglichen Notwendigkeit führen, Effizienzlöhne $E L_{j}$ zu zahlen, vom Einfluss der Gewerkschaften $E G_{j}$ in einer Organisation und vom Gewinnausschüttungsverbot $G V_{j}$, dem NPO unterliegen. Je höher die Notwendigkeit ist, Effizienzlöhne zu zahlen, desto wahrscheinlicher ist eine Verteilung der Renten durch die Zahlung höherer Löhne. Ebenso verhält es sich mit dem Einfluss der Gewerkschaften. Ein hoher gewerkschaftlicher Organisationsgrad führt zur Ausschüttung der Renten an die Arbeitnehmer/-innen. Auch das Gewinnausschüttungsverbot, dem NPO unterliegen, ermöglicht ein Ausschütten der Renten, da diese nicht an Eigentümer/-innen verteilt werden.

Zusammengefasst stellen sich die Bedingungen, die zur Verteilung von Renten führen, wie folgt dar:

$$
R S_{j}=r+s B W_{j}+t F Z_{j}+u O E_{j}+v O G_{j}+w M Z_{j}+x E L_{j}+y E G_{j}+z G V_{j}
$$

Aus der Kombination dieser Funktion mit der vorherigen, lässt sich die Lohnfunktion folgendermaßen zusammenfassen:

$$
\begin{aligned}
& W_{i j}=j+k I W_{i j}+l S A C_{j}+m B A C_{j}+n H K_{i}+o R_{j}+p D_{j}+ \\
& +q\left(r+s B W_{j}+t F Z_{j}+u O E_{j}+v O G_{j}+w M Z_{j}+x E L_{j}+y E G_{j}+z G V_{j}\right) \\
& W_{i j}=j+q r+k I W_{i j}+l S A C_{j}+m B A C_{j}+n H K_{i}+o R_{j}+p D_{j}+ \\
& +q s B W_{j}+q t F Z_{j}+q u O E_{j}+q v O G_{j}+q w M Z_{j}+q x E L_{j}+q y E G_{j}+q z G V_{j}
\end{aligned}
$$

Im Folgenden wird dargestellt, wie die Lohnfunktion empirisch geschätzt werden kann.

\section{2 Ökonometrische Implementierung}

Eine empirische Realisierung der oben genannten Lohnfunktion ist aufgrund fehlender Daten bzw. aufgrund der Tatsache, dass einige der oben genannten Maße nur sehr schwer messbar sind, nicht unmittelbar möglich. Deshalb müssen für einige Maße Proxys gesucht werden. Einige oben angegebene Größen können empirisch leider gar nicht umgesetzt werden, was ein Manko darstellt. 
Der intrinsische Wert $I W_{i j}$, den jedes Individuum der Arbeit in einer NPO, bzw. der Mission der NPO zuschreibt, ist nur schwer zu messen. Während Leete (2001: 143) dafür einen NPO Dummy in die Schätzfunktion einfügt, der das Ziel hat, Löhne im Nonprofit Sektor und im gewinnorientierten Bereich zu vergleichen, wird in dieser Arbeit ein anderer Weg gegangen. Hierfür werden ehrenamtliche Mitarbeiter/-innen als ein möglicher Proxy gesehen, um den intrinsischen Wert, den man der Arbeit in einer NPO zuschreibt, zu ,messen“. Die Arbeits-spendenhypothese unterstellt, dass Arbeitnehmer/-innen einer NPO bereit sind, auf einen Teil ihres Lohns zu verzichten und diesen Teil des Lohns zu „spenden“, da sie dem Gut einer NPO bzw. der Arbeit einer NPO einen Wert an sich zumessen. Ehrenamtliche Mitarbeit in einer NPO stellt einen extremen Fall der Arbeitsspende dar. An ihr kann gemessen werden, für wie „sozial wertvoll“ die Arbeit dieser NPO gesehen wird. Zusätzlich wird angenommen, dass das zahlenmäßige Verhältnis zwischen ehrenamtlichen Mitarbeiter/-innen und bezahlten Vollzeitäquivalenten mit zunehmendem beigemessenen intrinsischen Wert steigt. Deshalb werden sowohl ein Dummy, ob es ehrenamtliche Mitarbeiter/-innen in der Organisation gibt, als auch die Anzahl der ehrenamtlichen Mitarbeiter/-innen pro bezahltem Vollzeitäquivalent in die Schätzgleichung inkludiert. Ein weiteres Maß für den intrinsischen Wert der Arbeit wäre beispielsweise die Betriebszugehörigkeitsdauer eines Individuums. Leider stehen diese Daten nicht zur Verfügung.

Natürlich sind die beiden Variablen nur ein schwaches Maß für den intrinsischen Wert, den ein Individuum der Arbeit in einer NPO zuschreibt. Erstens ist dieser nicht für alle Personen in einer Organisation gleich groß. Zweitens ist nicht klar, ob bezahlte und ehrenamtliche Mitarbeiter/-innen den intrinsischen Wert der Arbeit der NPO gleich einschätzen. So ist es möglich, dass die beiden Gruppen von Beschäftigten sehr unterschiedliche Tätigkeiten verrichten oder unterschiedliche Persönlichkeitstypen sind. Beispielsweise könnten ehrenamtliche Mitarbeiter/-innen eine Kernaufgabe der Organisation verrichten und so das Ausmaß des intrinsischen Wertes viel unmittelbarer erkennen als ein bezahltes Individuum, das beispielsweise eine administrative Tätigkeit ausführt. Es ist außerdem nicht klar, ob die Annahme berechtigt ist, dass bezahlte Beschäftigte bewusst Arbeit im Nonprofit Sektor suchen oder ob sie vielmehr Arbeit suchen und diese zufällig im Nonprofit Sektor finden. Deshalb könnte drittens bezweifelt werden, ob bezahlte Mitarbeiter/-innen der Tatsache, im Nonprofit Sektor zu arbeiten, überhaupt intrinsischen Wert zumessen. Trotz der Einschränkungen werden der Ehrenamtlichen-Dummy und die Anzahl der ehrenamtlichen Mitarbeiter/-innen in der Organisationen als Proxys für den intrinsischen Wert der Arbeit einer NPO gesehen. Zusätzlich dient die ÖNACE Klassifizierung dazu, mögliche Unterschiede in der Einschätzung des intrinsischen Werts zu messen. Dabei wird angenommen, dass sich der zugemessene intrinsische Wert der Arbeit je nach Tätigkeitsbereich unterscheidet. 
Zur Beschreibung der sektorenspezifischen und der berufsspezifischen Arbeitsplatzcharakteristika $S A C_{j}$ und $B A C_{j}$, könnte eine Fülle an Variablen verwendet werden, die z.B. die Art der Tätigkeit oder die Arbeitszeiten beschreiben. Teilweise sind die Arbeitsplatzcharakteristika nicht messbar. Leider stehen zur Beschreibung der sektoren- und berufsspezifischen Arbeitsplatzcharakteristika im vorliegenden Datensatz (siehe Kapitel 7.3) nur wenige Daten zur Verfügung. Als grober Proxy werden einerseits die ÖNACE-Klassifizierung auf 6Steller Ebene (vgl. Statistik Austria o.J.) verwendet, andererseits die Variable „soziale Stellung“, die angibt ob das Individuum Arbeiter/-in, Angestellte/r, Vertrags-bedienstete/r oder Lehrling ist. Dabei wird unterstellt, dass Individuen, die in derselben Branche arbeiten, ähnlichen sektorenspezifischen Arbeitsbedingungen gegenüberstehen. Leider gibt es keine genaueren Stellenbeschreibungen als die sehr grobe Einteilung in Arbeiter/-innen und Angestellte. Deshalb muss die Annahme getroffen werden, dass die soziale Stellung Unterschiede berufsspezifischer Bedingungen aus-reichend beschreiben kann. Drittens geht eine Dummy-Variable, die angibt, ob eine Person vollzeit- oder teilzeitbeschäftigt ist, zur Messung der Arbeitsbedingungen und des -volumens ein. Dies ist zur Messung der Arbeitsplatzcharakteristika dann relevant, wenn hinsichtlich der Arbeitsbedingungen Unterschiede zwischen Vollzeit- und Teilzeitkräften bestehen. Als vierte Variablengruppe zur Messung sektoren- und berufsspezifischer Arbeitsplatzcharakteristika werden ein Ehrenamts-Dummy und die Anzahl der ehrenamtlichen Mitarbeiter/-innen herangezogen, insofern, als sie möglicherweise die Art der Tätigkeit bzw. der Verantwortungsbereiche der bezahlten Arbeitnehmer/-innen verändern. Wünschenswert wären genauere Angaben zur Art der Tätigkeit sowohl der unbezahlten als auch der bezahlten Arbeitskräfte. Letztere sind jedoch im Datensatz nur ungenau und schlecht ausgefüllt vorhanden, sodass sie nicht für die Berechnung herangezogen werden. Insgesamt wären genauere Angaben zu sektoren- und berufsspezifischen Arbeitsplatzcharakteristika wünschenswert.

Wie in Kapitel 3.2 beschrieben, stellt Humankapital $H K_{i j}$ die Summe aus Wissen und Fertigkeiten eines Arbeitnehmers bzw. einer Arbeitnehmerin dar, wobei üblicherweise zwischen allgemeinem und spezifischem Humankapital unterschieden wird. Es wird angenommen, dass zusätzliches Humankapital produktivitätssteigernd wirkt. Im Datensatz wären daher Informationen zur Ausbildung des Individuums sowie zur Arbeitserfahrung innerhalb der Branche und der jeweiligen Organisation ideal. Auch hier kann in den Daten nur eine Annäherung an das Humankapital der Arbeitnehmer/-innen über das Alter der Person erfolgen, da andere Informationen leider nicht zur Verfügung stehen. Deshalb wird in die Lohnfunktion das Alter eingefügt, zusätzliche außerdem das quadrierte Alter der Person. Wie in zahlreichen Untersuchungen üblich, wird unterstellt, dass die Lohnveränderungen mit zunehmendem Alter nicht linear verlaufen. Vielmehr wird davon ausgegangen, dass Lohnzuwächse mit höherem Alter kleiner werden (vgl. z.B. Franz 2006: 80). 
Außerdem wird ein Geschlechtsdummy in die Lohnfunktion aufgenommen, der einerseits etwaige Produktivitätsunterschiede oder Unterschiede im Humankapital aufgrund des Geschlechts abdecken soll. Ein Geschlechtsdummy als Indikator für Unterschiede im Humankapital ist dann notwendig, wenn davon ausgegangen werden muss, dass Männer und Frauen systematisch unterschiedliche Ausbildung erfahren haben. Aufgrund der relativ schlechten Datenlage zum individuellen Humankapital werden zusätzlich Variablen in die Schätzgleichung aufgenommen, die Humankapital auf Organisationsebene abbilden sollen: der Anteil der Teilzeitbeschäftigten in einer Organisation, der Anteil der geringfügig Beschäftigten und der Frauenanteil.

$R_{j}$ stellt die regionalen Bedingungen des Arbeitsplatzes dar. Dazu können z.B. die regionale Arbeitslosigkeit gezählt werden, die sich wiederum in der Lohnhöhe niederschlägt, oder das regionale Preisniveau. Als Proxys werden hierfür Bundesland Dummys in die Lohnfunktion eingebaut.

$D_{j}$ stellt Lohndiskriminierung dar, die aufgrund von Charakteristika, die nicht mit der Produktivität eines Individuums zusammenhängen, getroffen wird. Diskriminierung erfolgt häufig anhand des Geschlechts, des Alters, der Ethnizität oder Nationalität (vgl. Franz 2006: 340f.). Ob Diskriminierung stattfindet ist nur schwer direkt zu beobachten. In die Schätzgleichung werden Geschlecht und Alter als Variablen inkludiert, um etwaige Unterschiede in der Lohnhöhe, die aufgrund von Diskriminierung entstehen, herauszuarbeiten. Wird beiden Geschlechtern und allen Altergruppen gleiche Produktivität unterstellt, dann dienen die Variablen zur Überprüfung, ob Lohndiskriminierung stattfindet. Die Nationalität eines Arbeitnehmers bzw. einer Arbeitnehmerin wäre ebenfalls eine wichtige Variable, um etwaige Diskriminierung zu messen, liegt jedoch in den verwendeten Daten nicht vor.

Die Höhe der Renten in einer Organisation bestimmt sich aus dem Branchenwettbewerb, der Höhe der finanziellen Zuwendungen an die Organisation, der Organisationseffizienz, der Organisationsgröße und dem Maximierungsziel der NPO. Die Verteilung der Renten bestimmt sich durch die Notwendigkeit, Effizienzlöhne zu zahlen, dem Einfluss der Gewerkschaften und dem Gewinnausschüttungsverbot.

Der Branchenwettbewerb $B W_{j}$ wird über die ÖNACE-Dummys gemessen. Damit wird unterstellt, dass Umsätze und Gewinne branchenspezifisch sind.

Die Höhe der finanziellen Zuwendungen $F Z_{j}$, die nicht aus Umsatzerlösen resultieren, wird durch die Höhe der Spenden und der Subventionen an die Organisation gemessen.

Die Organisationseffizienz $O E_{j}$ wird ebenfalls über ÖNACE-Dummys gemessen, wenn unterstellt wird, dass die Organisationseffizienz branchenabhängig ist. Außerdem könnte angenommen werden, dass sich NPO in ihrer Organisations- 
effizienz von gewinnorientierten oder öffentlichen Unternehmen unterscheiden. $\mathrm{Da}$ jedoch ausschließlich NPO im Datensatz enthalten sind, kann auf die Verwendung eines Nonprofit-Dummys verzichtet werden. Die Höhe der Spenden kann eine weitere Indikatorvariable für Organisationseffizienz darstellen, wie in Kapitel 4 näher erläutert wurde.

Die Organisationsgröße $O G_{j}$ wird einerseits über die Anzahl der bezahlten Vollzeitäquivalente gemessen. Wie in Kapitel 4 argumentiert, greift ein Messen der Organisationsgröße in NPO ausschließlich anhand der bezahlten Mitarbeiter/-innen jedoch zu kurz, da in vielen Organisationen ehrenamtliche Mitarbeiter/-innen einen wesentlichen Teil der Arbeit verrichten. Aus diesem Grund ist die Aufnahme der Anzahl der ehrenamtlichen Mitarbeiter/-innen zur Messung der Organisations-größe wesentlich. Problematisch ist, dass im Fall der ehrenamtlichen Mitarbeiter/-innen nur eine Kopfzahl verwendet wird, da ein Berechnen von Vollzeitäquivalenten nur schwer möglich ist, weil die Daten zum Arbeitsausma $\beta$ ehrenamtlicher Mitarbeiter/-innen sehr ungenau sind. Der Grund dafür liegt im Wesen der ehrenamtlichen Arbeit, die teilweise unregelmäßig erfolgt und von der Organisationsleitung häufig nur schwer messbar bzw. abschätzbar ist. Von den Organisationen wurde angegeben, wie viele Personen sich mindestens einmal im Moment ehrenamtlich engagieren. Wie hoch das tatsächliche Stundenausmaß der ehrenamtlichen Arbeit ist, bleibt offen. Die Anzahl der ehrenamtlichen Mitarbeiter/-innen wirkt sich auch in der Verteilung vorhandener Renten aus, weshalb sie ein wichtiges Maß darstellen.

Ob vorhandene Renten verteilt werden hängt zum einen davon $a b$, ob es für die Organisation notwendig ist, Effizienzlöhne $E L_{j}$ zu zahlen. Diese Notwendigkeit wird in dieser Arbeit einerseits durch die Ehrenamts-Variablen, einem Dummy, der angibt, ob in der NPO ehrenamtliche Arbeitskräfte arbeiten und dem zahlenmäßigem Verhältnis der ehrenamtlichen Mitarbeiter/-innen zu den bezahlten Vollzeitäquivalenten in der Organisation, gemessen (vgl. dazu Kapitel 4). Die Notwendigkeit, Effizienzlöhne zu bezahlen, kann auch branchen- und regionenabhängig sein, wenn z.B. in bestimmten Branchen bzw. Regionen Mangel an Arbeitskräften mit bestimmter Qualifizierung vorherrscht, und, im Fall der Regionen, wenn Arbeitnehmer/-innen nicht ausreichend mobil sind. Aus diesem Grund, werden ÖNACE-Dummys und Bundesland-Dummys auch zur „Messung“ der Notwendigkeit, Effizienzlöhne zu zahlen, verwendet. Ein weiterer Indikator für die Notwendigkeit, Effizienzlöhne zu zahlen, kann die Anzahl der Mitarbeiter sein. Die Überwachungskosten können sich in Organisationen unterschiedlicher Größe unterscheiden, wobei diese Kosten in großen Organisationen vermutlich höher sind, da die Überwachung schwieriger wird. Deshalb werden die logarithmierten Vollzeitäquivalente ebenfalls als Maß für die Notwendigkeit, Effizienzlöhne zu zahlen, verwendet.

Der Einfluss der Gewerkschaften $E G_{j}$ wird in diesem Datensatz mittels der Frage, ob die Organisation einem oder mehreren Kollektivverträgen unterliegt, 
gemessen. Dazu werden zwei Dummys in die Schätzgleichung eingebaut, einerseits wenn die Organisation keinem Kollektivvertrag unterliegt, sich bei ihrem Lohnschema jedoch an einem Kollektivvertrag orientiert, andererseits wenn die Organisation keinem Kollektivvertrag unterliegt (und sich auch an keinem orientiert). Bezugsgröße ist jeweils eine Organisation, die einem oder mehreren Kollektivverträgen unterliegt.

Allen NPO ist gemein, dass sie einem Gewinnausschüttungsverbot $G V_{j}$ unterliegen, was ein Definitionskriterium von NPO ist (vgl. Badelt et al. 2007b: 7). Deshalb ist es nicht notwendig z.B. einen Dummy einzubauen, da nur NPO im Datensatz enthalten sind. Die Variable $G V_{j}$ geht daher nicht in die Schätzgleichung ein. Gleich verhält es sich mit dem Maximierungsziel $M Z_{j}$ von NPO. NPO maximieren nicht den Gewinn, sondern streben andere Ziele an.

Folgende Tabelle 5 gibt zusammenfassend eine Übersicht, wie die Variablen der Lohnfunktion durch Proxys ökonometrisch implementiert werden:

Tabelle 5: Ökonometrische Implementation der Variablen der Lohnfunktion

\begin{tabular}{l|l} 
Modellvariable & empirisch verwendete Variable \\
\hline intrinsischer Wert & Ehrenamt, ÖNACE \\
Sektorenspez. Arbeitsbedingungen & ÖNACE, soz.Stellung, Vollzeit/Teilzeit, Ehrenamt \\
Berufsspez. Arbeitsbedingungen & ÖNACE, soz.Stellung, Vollzeit/Teilzeit, Ehrenamt \\
Humankapital & Alter, Geschlecht, soz.Stellung d. Individuums, \\
& Anteil Teilzeit, Anteil Geringfügige, Frauenanteil in der \\
& Organisation \\
regionale Bedingungen & Bundesland \\
Diskriminierung & Alter, Geschlecht \\
Branchenwettbewerb & ÖNACE \\
finanzielle Zuwendungen & Spenden, Subventionen \\
Organisationseffizienz & ÖNACE, Spenden \\
Organisationsgröße & log. Vollzeitäquivalente, Ehrenamtliche \\
Maximierungsziel & Keine \\
Effizienzlöhne & Ehrenamt, ÖNACE, Bundesland, log. Vollzeitäquival. \\
Einfluss der Gewerkschaften & Kollektivvertragsdummys \\
Gewinnausschüttungsverbot & Keine \\
\hline
\end{tabular}

Quelle: eigene Darstellung

Durch Einsetzen der oben genannten Proxys für die einzelnen Variablen in der Lohnfunktion, lässt sich folgende empirisch überprüfbare Lohnfunktion aufstellen: 
(9) $\log W_{i j}=h\left(\begin{array}{l}\text { Alter, Alterquadrat, Geschlecht, Soz.Stellung, Vollzeit/Teilzeit, } \\ \text { Ehrenamtdummy, Anz. Ehrenamtliche, Spenden, Subvention, } \\ \text { Kollektivdummys, ÖNACE, Bundesland, } \\ \text { Ant. Teilzeit, Ant. Geringfügige, Frauenant.,VZÄ }\end{array}\right)$

Verkürzt dargestellt lautet die log-lineare Funktion der Schätzgleichung, die in dieser Arbeit zur Beantwortung der Forschungsfragen verwendet wird, wie folgt:

(10) $\log W_{i j}=\alpha+X \beta+Z \gamma+\varepsilon$

wobei $\log W_{i j}$ das logarithmierte Tageseinkommen eines Arbeitnehmers oder einer Arbeitnehmerin $i$ in der Organisation $j$ darstellt. Der Vektor $X$ umfasst individualspezifische Daten, nämlich Alter, Alter Quadrat, Geschlecht, „soziale Stellung“ sowie einen Vollzeit/Teilzeitdummy. Der Begriff „soziale Stellung“ umfasst mehrere Dummys, Angestellte/r, Lehrling sowie Vertragsbedienstete, wobei Arbeiter/-innen als Referenzkategorie dienen. Der Vektor Z umfasst alle organisationsspezifischen Variable und inkludiert einen Ehrenamts-Dummy, die Anzahl der freiwilligen Mitarbeiter/-innen pro Vollzeitäquivalent, die Höhe der Spenden und der Subventionen pro Vollzeitäquivalent, die die Organisation erhalten, zwei Kollektivvertragsdummys, eine Reihe von Dummys, die eine Einteilung der Organisationen nach der ÖNACE-Klassifikation vornehmen, acht Bundesländerdummys mit Wien als Referenzkategorie, den Anteil geringfügiger Beschäftigter an den Vollzeitäquivalenten, sowie den Anteil Teilzeitbeschäftigter und den Frauenanteil. Außerdem geht die logarithmierte Anzahl der Vollzeitäquivalente in die Schätzgleichung ein.

Die in der empirisch geschätzten Lohnfunktion verwendeten Variablen werden im folgenden Unterkapitel näher erläutert. Außerdem werden Aussagen über die vermuteten Vorzeichen der Koeffizienten getroffen.

\subsection{Spezifikation der Lohnfunktion und Hypothesenbildung}

Zum Zweck der Spezifikation der Lohnfunktion werden die verwendeten Variablen in drei Gruppen geteilt, einerseits in den Regressanden, andererseits in individualspezifische und organisationsspezifische Regressoren.

\subsubsection{Der Regressand: logarithmierte Bruttotageslöhne}

Als Regressand geht der logarithmierte Bruttotageslohn ein. Die Logarithmierung der Lohnvariable ist für Lohnfunktionen äußerst üblich (vgl. Lemieux 2006: 130). Allerdings bestehen in der Literatur auch Zweifel an der Richtigkeit der Logarithmierung der Einkommen im Modell. Für ein Logarithmieren sprechen nicht nur die ökonometrische Überlegung, dass die Ergebnisse durch ein Logarithmieren leichter interpretierbar sind oder dass Probleme durch nicht 
normalverteilte oder heteroskedaste Residuen durch ein Logarithmieren der Variable gelöst werden können, sondern auch eine theoretische Überlegung, da von einem multiplikativen Effekt der erklärenden Variablen und keinem additiven ausgegangen wird, der durch das Logarithmieren wiederum linear gemacht wird (Mincer 1958). Lemieux (2006: 130) hält fest, dass die Verteilung der Löhne in den meisten Bereichen auf der Verteilung am besten durch Log-Linearität beschrieben wird. Blackburn (2007: 73f.) hingegen, schätzt die Logarithmierung der Einkommensvariable als problematisch ein, da der Rückschluss des geschätzten Koeffizienten im logarithmierten Modell auf den des tatsächlichen Modells nicht einfach möglich ist. Betrachtet man ein einfaches Modell

(11) $w=e^{x^{\prime} \gamma}$

wobei $w$ wiederum den Lohn darstellt und $x$ einen Vektor unabhängiger Variablen, so findet sich in der empirischen Schätzung zumeist eine semi-logarithmische Schätzung:

(12) $\log (w)=x^{\prime} \beta+\varepsilon$ (vgl. Blackburn 2007:74).

Konsistente Schätzer von $\beta$ sind nicht auch für $\gamma$ konsistent. $\beta$ könnte irreführende Rückschlüsse über die marginalen Auswirkungen von der unabhängigen Variablen $x$ auf den Lohn $w$ im tatsächlichen Modell geben.

Trotz dieser Einschränkung geht wegen der höheren Verbreitung und zur besseren Vergleichbarkeit mit anderen empirischen Schätzungen der Lohn in seiner logarithmierten Form als abhängige Variable in die Schätzgleichung ein.

\subsubsection{Individualspezifische Variablen}

Die individualspezifischen Variablen umfassen Geschlecht, Alter, Arbeitsausmaß und ,soziale Stellung“. Sie sind in der Schätzgleichung inkludiert, um systematische beobachtete Unterschiede zwischen Arbeitnehmer/-innen zu berücksichtigen. Neben den oben genannten Variablen gäbe es auch andere wichtige individualspezifische Variablen, die aufgrund mangelnder Datenverfügbarkeit leider nicht berücksichtigt werden können. Dazu gehören z.B. Nationalität und Ethnizität, allgemeine und (betriebs-)spezifische Ausbildung sowie Betriebszugehörigkeit.

Alter stellt einen Proxy für allgemeines und betriebsspezifisches Humankapital dar oder kann ein Indikator für Lohndiskriminierung sein. Üblicherweise steigt die Lohnhöhe mit dem Alter, da vor allem das betriebsspezifische Humankapital wächst, wenn die Individuen im Unternehmen bleiben, womit das erwartete Vorzeichen für die Variable Alter positiv ist. Die Variable Alter quadriert wird eingefügt, da üblicherweise vermutet wird, dass die mit dem Alter erwartete Lohnzunahme kleiner wird. Folglich ist das erwartete Vorzeichen des Koeffizienten der Variable Alter quadriert negativ (vgl. Franz 2006: 80). 
Fügt man einen Geschlechtsdummy in die Lohngleichung ein, so wird allgemein erwartet, dass Männer mehr als Frauen verdienen. Gründe dafür können Unterschiede in der Produktivität sein, die auf Unterschieden in der Ausbildung, dem Beruf, der Branche, der Stetigkeit der Arbeitserfahrung oder der Arbeitszeit beruhen. Trotz Kontrolle für diese Unterschiede wird in der vorhandenen empirischen Evidenz eine Lohndifferenz zwischen Männern und Frauen gefunden, die unabhängig vom Zeitraum als auch vom untersuchten Land ist (siehe dazu z.B. Weichselbaumer, Winter-Ebmer 2005). Diese anhaltende Lohndifferenz resultiert aus der Lohndiskriminierung von Frauen (vgl. z.B. Cain 1986; Franz 2006: 342). Gunderson schätzt, dass Diskriminierung 5-15\% zu der gesamten Lohndifferenz zwischen Frauen und Männern beiträgt (vgl. Gunderson 2006: 10). Die Diskriminierung beruht unter anderem auf der Tatsache, dass Frauen eine andere Produktivität zugeschrieben wird. Da bei der Einstellung die Produktivität und die erwartete Betriebszugehörigkeit eines Individuums unbekannt sind, wird nach einem Merkmal gesucht, das Produktivität und die erwartete Betriebszugehörigkeit signalisieren kann. Dies kann neben der Ausbildung auch das Geschlecht sein, aufgrund dessen es zu Lohndiskriminierung kommt (vgl. Franz 2006: 341f.). Bei der Einfügung eines Frauendummys, der den Wert 1 annimmt, wenn die betreffende Person weiblich ist, 0, wenn sie ein Mann ist, wird demnach ein negatives Vorzeichen erwartet. Aus einem negativen Vorzeichen des Koeffizienten kann jedoch anhand der verwendeten Daten nicht abgelesen werden, ob es sich um Produktivitätsunterschiede zwischen den Geschlechtern oder um Lohndiskriminierung handelt, da wichtige Kontrollvariablen zur Messung der Produktivität eines Individuums im Datensatz fehlen.

Im Datensatz sind Angaben zur sozialen Stellung enthalten. Genauer werden die Dummys Angestellte/r, Lehrling und Vertragsbedienstete/r eingefügt, wobei Arbeiter/-innen als Referenzkategorie dienen. Gewöhnlich wird erwartet, dass Arbeiter/-innen aufgrund niedrigerer Produktivität weniger verdienen als Angestellte, das heißt, das erwartete Vorzeichen für einen Angestelltendummy ist positiv. Besser wäre die Angabe eines genauen Berufs einer Person. Hierfür fehlen jedoch Daten. Für Lehrlinge wird erwartet, dass sie weniger verdienen als Arbeiter/-innen und Angestellte, da sie über weniger Humankapital verfügen, das erwartete Vorzeichen ist folglich negativ. Vertragsbedienstete stehen im öffentlichen Dienst, sind jedoch in einem privatwirtschaftlichen Arbeitsverhältnis. Hier kann vermutet werden, dass sie Angestellten ähneln, d.h. gegenüber Arbeiter/-inne/n ein positives Vorzeichen zu erwarten ist.

Das Arbeitsausmaß gibt an, ob ein Individuum Vollzeit oder Teilzeit arbeitet, wobei ein Vollzeitarbeitsverhältnis mindestens 35 Wochenarbeitsstunden umfasst. Das erwartete Vorzeichen des Koeffizienten des Vollzeitdummys positiv, da die meisten Vollzeitkräfte absolut gesehen mehr verdienen als Arbeitnehmer/-innen, die Teilzeit arbeiten. 


\subsubsection{Organisationsspezifische Variablen}

In diese Gruppe fallen jene Variablen, die die Lohnhöhe auf Organisationsebene beeinflussen. Für alle Individuen innerhalb einer Organisation sind die Ausprägungen der organisationsspezifischen Variablen gleich.

Die nonprofit-spezifischen Variablen Ehrenamt, Spenden und Subventionen wurden im Kapitel 4 schon genauer erläutert, weshalb an dieser Stelle auf eine ausführliche Darstellung der erwarteten Vorzeichen verzichtet werden kann. Zur Darstellung des ehrenamtlichen Engagements innerhalb einer NPO wird einerseits eine Dummyvariable verwendet, die angibt, ob in einer Organisation ehrenamtliche Mitarbeiter/-innen arbeiten und andererseits die Anzahl der ehrenamtlichen Arbeitskräfte pro bezahltem Vollzeitäquivalent.

Spenden und Subventionen werden durch die Anzahl der Vollzeitäquivalente dividiert. $\mathrm{Zu}$ den Subventionen werden in diesem Teil der Arbeit auch Einnahmen aus Leistungsverträgen gezählt. Subventionen werden für die „grundsätzliche Zweckerfüllung" der NPO gewährt (Meyer 2007: 86). Immer mehr geht die öffentliche Hand jedoch dazu über, Subventionen durch leistungsvertragliche Arrangements zu ersetzen, in denen genaue Leistungen definiert werden, die die NPO gegen Bezahlung zu erbringen hat, wobei die Grenzen zwischen den beiden Vertragsbeziehungen fließend sind (vgl. Buchinger et al. 2008; Dimmel 2005; Meyer 2007; Schneider, Trukeschitz 2007; Zauner et al. 2006). Wegen der oft schlechten Trennbarkeit von Einnahmen aus Subventionen und Leistungsverträgen, werden letztere in diesem Teil der Arbeit zu den Subventionen gezählt.

A priori ist unklar, ob die erwarteten Vorzeichen der Koeffizienten der Ehrenamtsvariablen und der Spenden- und Sponsoringvariablen positiv oder negativ sind. Für einen positiven Einfluss der Ehrenamtsvariablen auf die Lohnhöhe spricht, dass vorhandene Renten auf weniger bezahlte Mitarbeiter/-innen verteilt werden im Vergleich zu NPO mit keinen oder weniger ehrenamtlichen Arbeitskräften, dass bezahlte Arbeitnehmer/-innen eine vergleichsweise höhere Qualifikation benötigen oder höhere Verantwortung tragen als Arbeitnehmer/-innen in NPO ohne freiwillige Mitarbeiter/-innen und dass Ehrenamtliche die Organisationsgröße erhöhen. Ein negatives Vorzeichen der Ehrenamtsvariablen könnte derart interpretiert werden, dass freiwillige Mitarbeiter/-innen in einer Organisation ein Indikator dafür sind, inwieweit die Tätigkeit der NPO als sozial wertvoll erachtet wird. Eine hohe Anzahl freiwilliger Arbeitskräfte kann ein Indikator dafür sein, dass auch bezahlte Individuen bereit sind, wegen hoher moralischer Befriedigung bzw. wegen eines hohen intrinsischen Werts auf einen Teil ihres Lohns zu verzichten. Löhne könnten auch deshalb niedriger sein, weil bezahlte Arbeitnehmer/-innen eine vergleichsweise geringe Qualifikation oder niedrige Verantwortung benötigen oder weil die Notwendigkeit, Effizienzlöhne 
zu zahlen sinkt. Außerdem können ehrenamtliche Mitarbeiter/-innen die Verhandlungsmacht der bezahlten Arbeitskräfte in Lohnverhandlung verringern.

Ein positives Vorzeichen der Koeffizienten der Spenden- und Subventionsvariable ist zu vermuten, weil sie auszuschüttende Renten erhöhen können oder weil hohe Spenden und Subventionen als ein Zeichen für besondere Fähigkeiten oder Qualitäten der Manager/-innen oder anderen Mitarbeiter/-innen verstanden werden können. Sie könnten sich negativ auf die erwartete Lohnhöhe auswirken, weil der Geldgeber bzw. die Geldgeberin Einfluss auf die Lohnhöhe haben kann oder weil durch niedrige Löhne Organisationseffizienz signalisiert werden soll.

Bezüglich der Kollektivvertragsdummys ist zu erwarten, dass jene Organisationen, die einem oder mehreren Kollektivverträgen unter-liegen, höhere Löhne zahlen als jene die dies nicht tun. Es wird zwischen den Gruppen ,unterliegt einem Kollektivvertrag“, „unterliegt keinem, orientiert sich jedoch im Lohnschema an einem Kollektivvertrag" und ,unterliegt keinem (und orientiert sich an keinem)" unterschieden. Generell wurde vielfach gezeigt, dass hoher Einfluss der Gewerkschaften zu höheren Löhnen führt (vgl. z.B. Farber 1986, Lewis 1986). Ein offensichtlicher Grund dafür ist die höhere Verhandlungsmacht, die den Arbeitnehmer/-inne/n durch gewerk-schaftliche Organisation zugute kommt. Es wird folglich vermutet, dass der Koeffizient für die Organisationen, die keinem Kollektivvertrag unterliegen, negativ ist, wenn als Referenzkategorie die NPO mit Kollektivvertrag vorliegen. Für die Variable, die angibt, dass die Organisation keine Kollektivvertrag unterliegt, sich jedoch an einem orientiert, kann angenommen werden, dass das Vorzeichen ebenfalls negativ ist, der Koeffizient jedoch kleiner ist als jener des zweiten Dummys in der Lohnfunktion.

Zur Beschreibung des Humankapitals auf Organisationsebene und zur zusätzlichen Kontrolle der Organisationsunterschiede liegen die Variablen Frauenanteil, Anteil der Teilzeitbeschäftigten an den Vollzeitäquivalenten und Anteil der geringfügig Beschäftigten vor. Hier wird erwartet, dass sich mehr geringfügig Beschäftigte negativ auf die Lohnhöhe auswirken. Auf Individualebene ist nur bekannt, ob eine Person Vollzeit oder Teilzeit arbeitet, nicht jedoch, wie viele Stunden, wenn sie Teilzeit beschäftigt ist. Ist nun der Anteil geringfügig Beschäftigter in einer Organisation sehr hoch, ist wiederum die Chance, dass ein Individuum im Datensatz, das Teilzeit beschäftigt ist, gering-fügig arbeit, sehr hoch. Die erwartete Lohnhöhe dieser geringfügig beschäftigten Person ist folglich niedriger als der einer Person, die teilzeit- aber nicht geringfügig beschäftigt ist. Für den Frauenanteil wird ein positives Vorzeichen vermutet, da in Organisationen mit hohem Frauenanteil angenommen werden kann, dass die durchschnittliche Qualifikation der Frauen höher ist als in NPO mit relativ wenig beschäftigen Frauen. Für den Anteil der Teilzeitbeschäftigten liegt a priori keine Vermutung vor. 
Die Organisationsgröße korreliert positiv mit der Lohnhöhe, wie bereits vielfach gezeigt wurde (vgl. z.B. Criscuolo 2000; Lallemand et al. 2005; Lallemand et al. 2007a; Medoff, Brown 1989; Oi, Idson 1999). Medoff und Brown (1989) geben eine Reihe von Gründen an, die dieses Phänomen erklären können. Erstens kann argumentiert werden, dass es hinsichtlich der Qualität der Arbeitnehmer/-innen und der Arbeits-bedingungen Unterschiede zwischen Organisationsgrößen gibt. Größere Unternehmen nehmen kompensierende Lohnzahlungen vor, da sie schlechtere Arbeitsbedingungen (wie z.B. monotonere Arbeit) anbieten. Es ist anzunehmen, dass diese Firmen Arbeitnehmer/-innen mit höherer Qualifikation bzw. Qualität beschäftigen.

Der Effizienzlohntheorie zufolge kann zweitens behauptet werden, dass Arbeitnehmer/-innen in größeren Organisationen schwerer zu über-wachen sind. Die Organisation sucht daher vorwiegend Arbeit-nehmer/-innen mit höherer Qualität, denen unterstellt wird, dass sie weniger shirking betreiben, oder aber die Organisation bezahlt (höhere) Effizienzlöhne, um shirking zu verhindern (vgl. Medoff, Brown 1989: 1029).

Andere Erklärungen können unter dem Begriff institutionelle Erklärungen zusammengefasst werden (vgl. Medoff, Brown 1989: 1031). Größere Unternehmen haben für gewöhnlich einen größeren Marktanteil bzw. größere Marktmacht, was zu zusätzlichen Renten führen kann, die konsequenterweise an Beschäftigte des Unternehmens durch Rent-Sharing verteilt werden. Außerdem führen die Autoren an, dass Unternehmen durch höhere Löhne gewerkschaftliche Organisation innerhalb des Unternehmens verhindern wollen, was für Österreich aber ein weniger relevanter Grund ist, da gewerkschaftliche Organisation auf überbetrieblicher Ebene geschieht. Medoff und Brown (1989: 1056) finden in ihren Untersuchungen, dass die Lohnhöhe auch dann noch positiv mit der Unternehmensgröße korreliert, wenn für Arbeitsbedingungen und Arbeitnehmercharakteristika kontrolliert wird.

ÖNACE Dummys, die eine Kategorisierung der Tätigkeit der Organisation nach Branchen vornehmen, werden in die Schätzgleichung eingefügt, um Unterschiede in den Arbeitsbedingungen, dem Branchenwettbewerb, der Organisationseffizienz und der Notwendigkeit, Effizienzlöhne zu zahlen, zu berücksichtigen. Unterschiedliche Methoden der Produktion von Waren und Dienstleistungen können ebenso durch Branchendummys abgebildet werden (vgl. z.B. Criscuolo 2000). Weiters bilden sie eventuell einen unterschiedlich zugeschriebenen intrinsischen Wert der Tätigkeit ab, wenn er sich zwischen Branchen unterscheidet. Normalerweise verfügen Arbeit-nehmer/-innen in kapitalintensiven Branchen über höhere Löhne, Arbeitnehmer/-innen im Service Sektor über niedrigere. Insgesamt zeichnen sich bestimmte Branchen durch hohe Anforderungen an die durchschnittliche Qualifikation der Arbeitnehmer/-innen aus, sodass für dieser Branchen auch ein positives Vorzeichen des Koeffizienten erwartet wird. Referenzkategorie ist die Branche „Sozialwesen“. 
Bundeslandsdummys werden verwendet, um regionale Unterschiede im Preisund Lohnniveau und in den Arbeitslosenraten einzufangen. Wien gilt als Referenzkategorie. Bei einer Betrachtung der Medianeinkommen nach Bundesländern in Österreich, fällt kein offensichtliches West-Ost- oder Ost-West-Gefälle auf (vgl. Statistik Austria 2007: 232f.). Über dem österreichweiten Medianeinkommen liegen die Medianeinkommen der Bundesländer Burgenland, Niederösterreich, Oberösterreich, Vorarlberg und Wien, die anderen Bundesländer liegen darunter. Dieses Ergebnis wird daher auch für den vorliegenden Datensatz vermutet. Da Wien Referenzgröße ist, kann das erwartete Vorzeichen für die Bundesländer Burgenland, Niederösterreich, Oberösterreich und Vorarlberg positiv oder negativ sein, für die restlichen Bundesländer ist es negativ. Einschränkend ist zu erwähnen, dass die Einkommensstatistik der Statistik Austria (2007), auf der die Erwartungen beruhen, auch andere Einkünfte als den Lohn berücksichtigt.

Folgende Tabelle 6 fasst die erwarteten Vorzeichen der Koeffizienten in der empirisch geschätzten Lohnfunktion zusammen:

\section{Tabelle 6: erwartete Vorzeichen der Koeffizienten der Lohnfunktion}

\begin{tabular}{|c|c|c|}
\hline Variable & Bezugsgröße & erwartetes Vorzeichen \\
\hline Alter & & + \\
\hline Alter quadriert & & - \\
\hline Weiblich & männlich & - \\
\hline Lehrling & & - \\
\hline Angestellte/r & Arbeiter & + \\
\hline Vertragsbedienstete/r & & + \\
\hline Volllzeit beschäftigt & Teilzeit beschäftigt & + \\
\hline Ehrenamt Dummy & keine & $+1-$ \\
\hline Anzahl Ehrenamtliche/VZÄ & & +1 \\
\hline Spenden/VZÄ & & + \\
\hline Subventionen/VZÄ & & $+1-$ \\
\hline kein Kollektivvertrag, aber Orientierung & unterliegt KV & - \\
\hline kein Kollektivvertrag & unterliegt KV & . \\
\hline ÖNACE & Sozialwesen & $+1-$ \\
\hline Bundesland & Wien & $\begin{array}{l}\text { +1- für BL, NÖ, OÖ, vO und - für } \\
\text { ST K. S. T }\end{array}$ \\
\hline Anteil Telizeitb. & & $+1-$ \\
\hline Anteil Geringfügig & & - \\
\hline Anteil Frauen & & + \\
\hline Log. VZÄ & & + \\
\hline
\end{tabular}

Quelle: eigene Darstellung 
Vor der Darstellung der Ergebnisse wird die Datengrundlage vorgestellt. Da es sich dabei um zusammengesetzte Arbeitnehmer-Arbeitgeberdaten (LEE-Daten) handelt, wird zuerst auf diese Besonderheit genauer eingegangen, bevor anschließend ein deskriptiver Überblick über die verwendeten Daten gegeben wird.

\subsection{Beschaffenheit von LEE-Daten und deren Vorteile für arbeitsökonomische Analysen}

Zur Schätzung der Lohnfunktionen werden Arbeitnehmer-Arbeitgeberdaten bzw. Linked Employer-Employee (LEE) Daten verwendet. Derartige Datensätzen werden teilweise auch Matched Employer-Employee Daten genannt und beinhalten Angaben über Arbeitnehmer/-innen, die mit Daten über deren Arbeitgeber/-innen verknüpft sind. Derartige Daten werden vor allem für Fragestellungen herangezogen, die die Interaktion von Betrieben und Beschäftigten betreffen.

Lange Zeit wurden arbeitsökonomische Fragestellungen entweder mit Haushalts- oder Betriebsdaten beantwortet. Nach Entwicklung der Humankapitaltheorie durch u.a. Mincer (1958), Schultz (1963) und Becker (1975) konzentrierte sich die Arbeitsmarktökonomie bei der Schätzung von Lohnfunktionen auf Individualdatensätze, die die Einflüsse der Humankapitalausstattung und verschiedener anderer Merkmale eines Individuums auf die Lohnhöhe untersuchten. Später nahm das Interesse an arbeitsnachfrageseitigen Einflussfaktoren auf die Lohnhöhe zu, weshalb in der Arbeitsökonomie zunehmend auch Betriebsdaten zur Schätzung von Lohnfunktionen verwendet wurden. Dabei musste jedoch auf das Einbeziehen von Humankapitalfaktoren aus Datengründen verzichtet werden (vgl. Klodt 2000: 98). Die meisten Ergebnisse am Arbeitsmarkt resultieren jedoch aus dem Zusammen-wirken beider Seiten des Marktes (vgl. Hamermesh 1999: 26). LEE-Daten ermöglichen es, sowohl individuen- als auch organisations-spezifische Effekte bei der Schätzung der Lohnfunktion mit einzubeziehen. Arbeitsmärkte können somit von der Angebots- und gleichermaßen von der Nachfrageseite betrachtet werden, weshalb Haltiwanger et al. die Bedeutung von LEE-Datensätzen für die Arbeits-marktökonomik auch für die $\mathrm{Zu}$ kunft sehr hoch einschätzen (vgl. Haltiwanger et al. 1999: xxi). Derartige Arbeitnehmer-Arbeit-geberdatensätze waren bislang kaum verfügbar, weshalb viele Frage-stellungen, die die (Firmen-)Lohnpolitik und Lohnstruktur betreffen, empirisch nicht beantwortbar waren (vgl. Stephan 2001: 14). Zur Zeit kann die Verwendung von LEE-Datensätzen noch als eine eher junge Entwicklung eingeschätzt werden, weltweit sind nur wenige derartige Datensätze vorhanden, wo- 
bei LEE-Daten als ein ,rasant wachsendes Forschungsgebiet" (Bellmann et al. 2002: 21) einzustufen sind und für die zukünftige Entwicklung ,entscheidend für den Fortschritt auf den Forschungsgebieten der Lohnstruktur- und der Humankapitaltheorie" (Bellmann et al. 2002: 21) sind.

Viele der vorhandenen LEE-Datensätze beinhalten meist Daten aus dem gewinnorientierten produzierenden Gewerbe oder der gewinnorientierten Dienstleistungsbranche. NPO haben bisher, meines Wissens nach, nur in einzelnen Branchen Eingang in derartige Datensätze gefunden oder konnten bei bestehenden Datensätzen nicht extra ausgewiesen werden. Ein Hauptgrund dafür ist die in vielen Ländern mangelnde Erfassung und Kennzeichnung von NPO durch die offiziellen statistischen Ämter (vgl. z.B. Schneider et al. 2006). Der Datensatz, der in der Dissertation zur Bearbeitung der Forschungsfragen verwendet wird, ist daher höchst innovativ.

Bestehende Studien, die Löhne im Nonprofit Sektor untersuchen, verwenden entweder Individualdaten (vgl. Goddeeris, 1988; Leete 2001; Preston 1989; Preston 1990; Shackett, Trapani 1987; Ruhm, Borkoski 2003; Weisbrod 1983) oder Daten auf Organisationsebene (vgl. Preston 1988). Einige Studien verwenden LEE-Daten, wobei hierbei meist nur ein bestimmter Aktivitätssektor untersucht wird, wie z.B. Kindergärten oder Alten- und Pflegeheime (vgl. Borjas et al. 1983; Holtmann, Idson 1993; Mocan, Tekin 2003; Noguchi, Shimizutani 2007).

\subsection{Methodische Erfordernisse bei hierarchischen Datenstrukturen}

Die untersuchten Daten zeichnen sich durch eine besondere Struktur aus. Sie bestehen aus Individualdaten, die mit Organisationsdaten verknüpft wurden. Sie zeichnen sich insofern durch eine hierarchische Struktur aus, als jeweils Individuen in Organisationen gruppiert sind.

Mithilfe einer einfaktoriellen Varianzanalyse kann das Ausmaß von Gruppenunterschieden (in dem Fall: Unterschieden zwischen Organisationen) bestimmt werden. Unterscheiden sich die Individuen in ihren Merkmalen stark zwischen den Organisationen, aber nur wenig innerhalb der Organisationen, muss diese Datenstruktur bei weiterführenden Berechnungen beachtet werden und dafür eine Korrektur vorgenommen werden (vgl. Snijders, Bosker 1999: 21f.). Dargestellt werden die Ergebnisse der Varianzanalyse der Variablen „Bruttotageslohn“ und „Organisation“ bzw. „logarithmierter Brutto-tageslohn“ und „Organisation". In diesem Fall wird also getestet, ob sich die Bruttotageslöhne bzw. die logarithmierten Bruttotageslöhne zwischen den Organisationen unterscheiden. Die Nullhypothese des F-Tests lautet, dass keine Gruppendifferenzen bestehen, bzw. dass die Varianz zwischen den Faktorstufen (between-group variance) gleich Null ist. 
In Tabelle 7 sind die Ergebnisse der beiden Varianzanalysen zu sehen. Dabei wird angenommen, dass der Bruttotageslohn nicht unabhängig von der jeweiligen Organisation ist, in der der Bruttotageslohn verdient wird. Abgebildet sind jeweils die Summe der quadrierten Abweichungen SS, die Freiheitsgrade df, die mittlere quadratische Abweichung MS, der F-Wert und die Wahrscheinlichkeit, dass der empirische F-Wert größer ist als der theoretische. ${ }^{29}$

Es zeigt sich, dass in beiden Varianzanalysen die Nullhypothese des F-Tests, nämlich dass bezüglich des Bruttotageslohns keine durch die Organisation hervorgerufenen Unterschiede bestehen, abgelehnt werden muss.

\section{Tabelle 7: Ergebnisse der Varianzanalyse}

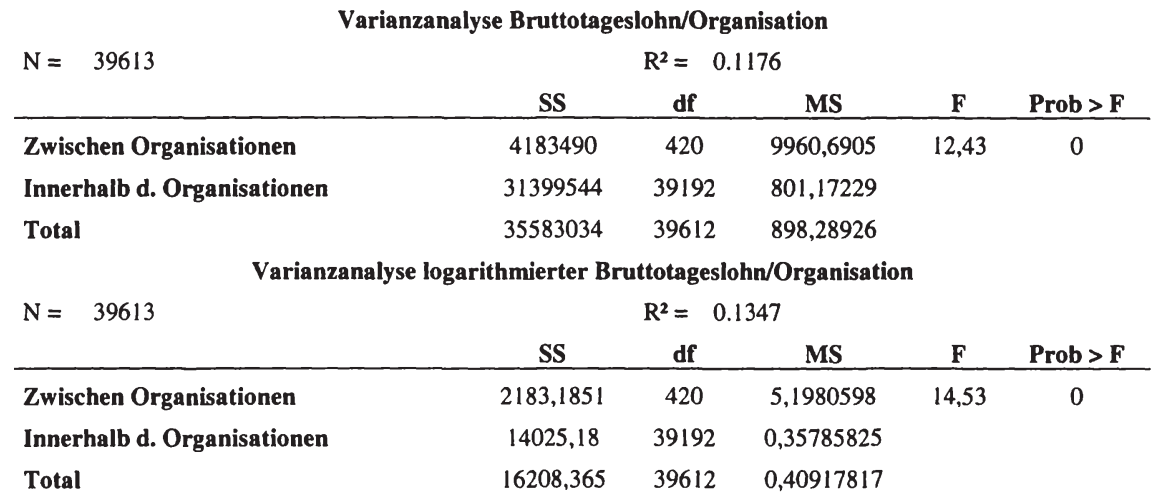

Quelle: NPO-Steuer-Daten 2006, eigene Berechnungen

Zusätzlich wurde der Intraklassenkorrelationskoeffizient $\rho$ (intraclass correlation coefficient) eines sogenannten „leeren Multi-level Modells“ (empty model) berechnet, das nur eine Konstante und keine erklärenden Variablen beinhaltet, wobei der logarithmierte Bruttotageslohn als Regressand dient. Der Intraklassenkorrelationskoeffizient wird berechnet als:

(13) $\rho=\frac{\tau^{2}}{\tau^{2}+\sigma^{2}}$ (vgl. Snijders, Bosker 1999: 17)

wobei $\tau^{2}$ die Varianz zwischen den Gruppen (Organisationen) darstellt und $\sigma^{2}$ die Varianz innerhalb der Gruppen (Organisationen). Ist $\rho$ sehr niedrig, kann dies so interpretiert werden, dass die Gruppen (Organisationen) nur wenig zur Erklärung der Gesamtvarianz beitragen, d.h. dass keine besondere hierarchische Struktur vorliegt. In diesem Fall beläuft sich das $\rho$ auf 0.31 , was als einigermaßen hoch eingeschätzt werden kann.

29 Für eine Einführung in die Varianzanalyse siehe z.B. Backhaus et al. (2000: 70-103). 
Die Individualeinkommensdaten sind demnach nicht unabhängig von der jeweiligen Organisation. Darauf muss in den darauffolgenden Schätzungen Rücksicht genommen werden. Die hierarchische Struktur kann auf mehrere Arten berücksichtigt werden: Einerseits kann sie durch eine Korrektur der Standardfehler berücksichtigt werden, wofür robuste Standardfehler berechnet werden. Andererseits kann auf die spezielle hierarchische Struktur durch die Anwendung von Multilevel-Analysen eingegangen werden (vgl. z.B. Snijders, Bosker 1999). $\mathrm{Da}$ aber die hierarchische Struktur nicht von primärem Interesse zur Beantwortung der Forschungsfrage ist, wird hiervon abgesehen.

\subsection{Der LEE-Datensatz „NPO-Steuer-Daten 2006“}

Die für die Dissertation zusammengestellten Daten werden in Folge kurz „NPOSteuer-Daten 2006“" genannt. Sie bestehen aus Querschnittsdaten von österreichischen NPO und deren Arbeitnehmer/-innen. Die organisationsspezifischen Angaben stammen aus einer schriftlichen Befragung von österreichischen NPO mit mindestens einem bezahlten Arbeitnehmer bzw. einer bezahlten Arbeitnehmerin, die im Jahr 2006 vom Institut für Sozialpolitik der Wirtschaftsuniversität Wien gemeinsam mit der Statistik Austria durchgeführt wurde. Insgesamt wurden 5104 Organisationen kontaktiert, 947 Fragebögen wurden retourniert, wobei in dieser Zahl auch gewinnorientierte und öffentliche Organisationen inkludiert sind, die als Kontrollgruppen angeschrieben wurden. Das entspricht einer Antwortrate von ca. $18,5 \%$. Die Organisationen sind in verschiedenen für den Nonprofit Sektor relevanten Aktivitätsbereichen tätig. Schulen, Krankenhäuser und einzelne Pfarren wurden jedoch nicht gesondert kontaktiert. In der Befragung wurde eine Vielzahl an Themen behandelt, Schwerpunkte liegen beim Tätigkeitsbereich der Organisation, bei der Beschäftigung, bei Einnahmen und Ausgaben der Organisation, sowie bei der Einschätzung über den Entwicklungstrend der Organisation in Hinsicht auf Wettbewerb, Kund/-inn/en, Einnahmen, Ausgaben und Mitgliedern. ${ }^{30}$ Die Organisationsdaten beziehen sich jeweils auf das Kalenderjahr 2005 bzw. fallweise auf den Stichtag 31.12.2005.

Die arbeitnehmerspezifischen Daten werden der Einkommens-steuerstatistik entnommen und wurden durch die Statistik Austria zur Verfügung gestellt. Verknüpft wurden die Daten mittels der SID-Nummer, die jeder Organisation zugeordnet ist. ${ }^{31}$ Verwendet wurden die Angaben der Jahreslohnzettel (L16), die jede

30 Für eine genauere Darstellung der Befragung, sowie der deskriptiven Statistik und Hochrechnungen für den gesamten NPO Sektor siehe Haider, Leisch, Schneider, Stöger (2008).

31 Ausnahmen bilden die öffentlichen Organisationen, denen keine SID-Nummer zugeordnet ist. 
Organisation für alle Arbeitnehmer/-innen ausstellen muss und jährlich an das Finanzamt übermittelt. Im Jahreslohnzettel müssen sämtliche Lohnzahlungen des vergangenen Kalenderjahres ausgewiesen werden. ${ }^{32}$ Für Werkvertragsnehmer/-innen oder freie Dienstnehmer/-innen wird kein Lohnzettel ausgestellt, d.h. diese Kategorie bezahlter „Beschäftigter ${ }^{\text {‘33 }}$ ist nicht in den Daten inkludiert.

Auf Individualebene stehen folgende Daten zur Verfügung:

- Einkommen (Brutto- und Nettodaten)

- Bezugsdauer des Einkommens in Tagen

- Geschlecht

- Alter

- Dummy Vollzeit/Teilzeitbeschäftigung

- Soziale Stellung (Lehrling, Arbeiter/-in, Angestellte/-r, Beamter/Beamtin, Vertragsbedienstete/-r, Pensionist/-in nach dem ASVG, Beamter/Beamtin in Ruhe, Bezieher/-in einer sonstigen Pension

Das Bruttoeinkommen stellt das Jahreseinkommen inklusive allen Einmal- und Sonderzahlungen dar. Deshalb wurden für die folgenden Berechnungen eine Einkommensgröße herangezogen, die einmalige Zahlungen wie Abfertigungszahlungen nicht beinhaltet ${ }^{34}$. Sehr wohl beinhaltet sie Überstundenzahlungen, das 13. und 14. Monatsgehalt, etc. Das Einkommen wurde durch die Bezugsdauer in Tagen dividiert, um vergleichbare Werte für Arbeitnehmer/-innen unterschiedlich langer Beschäftigungsdauer herzustellen. ${ }^{35}$

Für die weiterführenden Analysen in der Dissertation wurden einige Organisationen ausgeschlossen. Viele Kindergärten wurden ausgeschlossen, da für sie keine Angaben zu Einnahmen und Ausgaben zur Verfügung standen. Zum Beispiel wurde des weiteren ein Fußballverein der österreichischen Bundesliga ausgeschlossen, da er im Vergleich zu den anderen Organisationen vor allem in Bezug auf das Einkommen der bezahlten Arbeitskräfte des Vereins als extremer Ausreißer bezeichnet werden kann. Außerdem wurden die Informationen von Zweigstellen von Organisationen, die einen Fragebogen retournierten, denen der

32 vgl. z.B. http://www.help.gv.at/Content.Node/99/Seite.990043.html

33 Genau genommen sind Werkvertragsnehmer/-innen nicht bezahlt beschäftigt, sondern gelten als Vertragspartner/-innen mit der Organisation.

34 Am Lohnzettel wird der Betrag unter „Steuerfreie bzw. mit festen Sätzen versteuerte Bezüge gemäß $§ 67$ Abs.3 bis 8, vor Abzug der SV-Beiträge“ ausgewiesen.

35 Ein in der empirischen Literatur üblicher Stundenlohn konnte aufgrund fehlender Angaben zu den Arbeitsstunden nicht ermittelt werden. 
Zentralen zugewiesen. Organisationen, denen keine SID-Nummer zugewiesen werden konnte, wurden ebenfalls ausgeschlossen.

Ausgeschlossen wurden jene Individuen, die Pensionisten bzw. Pensionistinnen nach dem ASVG, Beamte in Ruhe oder Bezieher/-innen einer sonstigen Pension waren, da sie keine aktiven Arbeitnehmer/-innen der Organisationen sind. Des weiteren werden jene Individuen ausgeschlossen, bei denen keine Angabe zum Geschlecht besteht oder dazu, ob sie vollzeit- oder teilzeitbeschäftigt waren. Außerdem wurden aus Plausibilitätsgründen alle Fälle ausgeschlossen, bei denen das Tageseinkommen ${ }^{36}$ für Teilzeitkräfte weniger als fünf Euro ausmachte und für Vollzeitkräfte weniger als 20 Euro. ${ }^{37}$ Es wird vermutet, dass bei Fällen, die ein Tageseinkommen unter diesen beiden Grenzen aufweisen, die Bezugsdauer nicht korrekt angegeben wurde. Leider erlauben Lohnzetteldaten keine genauere Unterscheidung nach Wochenarbeits-zeit, da lediglich ein Dummy vorhanden ist, der angibt, ob die Person vollzeit- oder teilzeitbeschäftigt ist. Eine Aussage darüber, ob eine teilzeitbeschäftigte Person eine oder 34 Stunden pro Woche arbeitet, ist jedoch nicht möglich. Da sich der Nonprofit Sektor insgesamt durch eine hohe Bedeutung der Teilzeitarbeitskräfte auszeichnet, werden sie bewusst nicht aus dem Datensatz ausgeschlossen (vgl. Trukeschitz 2006: 206 und 221f. für den Sektor der sozialen Dienste). Dadurch bedingt, wurde die Untergrenze der plausiblen Fälle für die Teilzeitkräfte sehr niedrig bei fünf Euro Tageseinkommen festgesetzt. Es ist allerdings nicht auszuschließen, dass der Datensatz noch immer unplausible Fälle beinhaltet, bei denen die Angabe über die Bezugsdauer nicht korrekt ist.

Für die weiterführenden Berechnungen im ersten Teil der Dissertation wurden schlussendlich 421 NPO verwendet, in denen 39.613 Individuen arbeiten, womit $8,25 \%$ aller ursprünglich kontaktierten Organisationen erfasst sind.

\subsection{Umgang mit fehlenden Werten}

Fast immer enthalten Befragungsdaten fehlende Werte, die z.B. darauf zurückzuführen sind, dass Individuen bestimmte Fragen nicht beantworten können oder wollen. Es gibt mehrere Arten von fehlenden Werten, ebenso zahlreich sind die Möglichkeiten, wie in quantitativen Schätzungen mit diesen umzugehen ist. Auch die „NPO-Steuer-Daten 2006“ beinhalten fehlende Werte, weshalb im

36 Einkommen ohne Abfertigungen dividiert durch die Bezugsdauer

37 Die Berechnungen wurden auch mit anderen Spezifikationen, nämlich mit der Untergrenze von 7 Euro (Teilzeit) und 23 Euro (Vollzeit), durchgeführt. 
Folgenden ein kurzer Überblick über mögliche Arten des Umgangs mit fehlenden Werten gegeben wird $^{38}$ :

\subsubsection{Arten fehlender Werte}

Üblicherweise wird zwischen drei Arten von fehlenden Werten unterschieden:

- MCAR (Missing Completely at Random): Diese fehlenden Werten sind vollkommen zufällig, d.h. die Tatsache, dass der Wert fehlend ist, ist sowohl von der betroffenen, zum Teil fehlenden, Variable, als auch von allen anderen Variablen im Datensatz unabhängig.

- MAR (Missing at Random): Obwohl diese Art der fehlenden Werte als ,zufällig fehlend“ bezeichnet wird, fehlen die Werte hier eigentlich nicht zufällig, was irreführend ist. Bei dieser Kategorie sind Werte fehlend, abhängig von einer anderen Variable im Datensatz. Ein simples Beispiel für ein Vorliegen von fehlenden Werten der Kategorie MAR ist, wenn z.B. vermehrt Frauen die Frage nach dem Gewicht nicht beantworten.

- NMAR (Not Missing at Random) ${ }^{39}$ : Hier ist der fehlende Wert ebenfalls nicht zufällig. Ob ein Wert fehlend ist, hängt vom tatsächlichen Betrag der Variable ab. Der fehlende Wert hängt dabei systematisch mit der im Mittelpunkt stehenden Variable zusammen. Wenn beispielsweise bei der Frage nach dem Einkommen vermehrt hohe Einkommen fehlen, liegt die Kategorie NMAR vor. Sehr oft tritt der Fall auf, wenn Daten nach eigenen Angaben der Individuen bzw. Organisationen gewonnen werden, sodass es unter Umständen zu einem Selbstselektionsproblem kommen kann.

Während die ersten beiden Kategorien von fehlenden Werten als ignorierbar bezeichnet werden, ist letztere für weiterführende Berechnungen problematisch (vgl. Griliches 1986: 1486). NMAR Daten können zum Teil mithilfe geeigneter Methoden korrigiert werden (vgl. Greene 2003: 59 und 756ff.). Allerdings ist zu bedenken, dass auch der ,ignorierbare" Fall nicht immer ignorierbar ist, da streng genommen nur bei MCAR Daten das einfache Ausschließen von Fällen mit fehlenden Werten nicht zu Verzerrungen führt. Um Verzerrungen bei den Ergebnissen zu vermeiden, ist es bei der Wahl einer passenden Alternative entscheidend, um welchen Typ von fehlenden Werten es sich handelt. Leider gibt es keinen statistischen Test, der eine derartige Entscheidung untermauern kann. Vielmehr muss die Entscheidung aufgrund inhaltlicher Überlegungen durch den Forscher bzw. die Forscherin getroffen werden.

38 Eine ausführliche Darstellung zum Umgang mit fehlenden Werten findet sich z.B. in Schafer (1997), Little und Rubin (2002) oder Allison (2002).

Teilweise ist auch die Abkürzung MNAR (Missing Not at Random) gebräuchlich.

Astrid Haider - 978-3-631-75377-4 


\subsubsection{Möglicher Umgang mit fehlenden Werten}

Generell kann zwischen vier Möglichkeiten unterschieden werden, wie Verzerrungen durch fehlende Werte in Regressionsanalysen vermieden werden können:

- Fallausschluss: Hier werden die Fälle, die fehlende Werte aufweisen, aus den Daten für einzelne Berechnungen ausgeschlossen, sodass sich die Fallzahl reduziert. Die meisten Statistikprogramme haben diese Möglichkeit als Voreinstellung, so auch das Statistikprogramm STATA, das für die Berechnungen im empirischen Teil der Arbeit verwendet wird.

- Missing Dummy: Hier wird der fehlende Wert durch einen häufigen Wert oder 0 ersetzt und zusätzlich ein Dummy angelegt, der den Wert 1 annimmt, wenn der Wert fehlend ist und 0, wenn er nicht fehlend ist. Dieser Dummy wird dann in das Regressionsmodel zusätzlich aufgenommen (vgl. Greene 2003: 60).

- Einfache Imputation: Generell wird bei einer Imputation versucht, fehlende Werte durch plausible Substitute zu ersetzen. Einfache Imputation umfasst mehrere Möglichkeiten, wie das Ersetzen der fehlenden Werte durch den Mittelwert, den Median oder den Modus der jeweiligen Variable. Außerdem ist es möglich, die Variable mit den fehlenden Werten auf andere erklärende Variablen zu regressieren und die fehlenden Werte durch die aus der Regression geschätzten Werte zu ersetzen. Weitere regressionsanalytische Verfahren umfassen Stochastische Regression, $\mathrm{EM}^{40}$-Imputation oder das Hotdeck-Verfahren. Beim Hotdeck-Verfahren werden einzelne Fälle, die fehlende Werte enthalten, mit ähnlichen Fällen anhand relevanter Variablen verglichen. Von diesen Fällen werden in Folge die Werte von diesen übernommen und statt den fehlenden Werten eingesetzt. Für Längsschnittdaten gibt es die Möglichkeit den Wert aus der Vorperiode einzusetzen und weiterzutragen (vgl. Scheffer 2002).

- Multiple Imputation: Bei der multiplen Imputation wird ein fehlender Wert mehrfach - üblicherweise 3 bis 10 mal - imputiert, sodass mehrere Datensätze entstehen. Diese Datensätze werden in Folge für weiterführende Analysen einzeln herangezogen und die daraus gewonnen Ergebnisse werden kombiniert. Zusätzlich werden für die Ergebnisse Konfidenzintervalle berechnet. Mehrfache

40 Expectation Maximisation Imputation: siehe dazu z.B. Griliches (1986: 1492) oder Little und Rubin (2002). 
Imputation soll die Variabilität und die Unsicherheit, die aus dem Imputationsprozess entsteht, besser abbilden (vgl. z.B. Horton, Kleinman 2007: 80). Auch hier kann zwischen einer Reihe unterschiedlicher Verfahren unterschieden werden, auf eine genaue Darstellung wird in dieser Arbeit jedoch verzichtet ${ }^{41}$.

Fallausschluss hat den Nachteil, dass unter Umständen sehr viele Datenfälle ausgeschlossen werden, wenn sich die fehlenden Werte auf unterschiedliche Variablen verteilen. Nur wenn es sich um MCAR handelt, verzerrt ein Ausschluss der Fälle mit fehlenden Werten die Daten nicht, ansonsten können durch Fallausschluss Verzerrungen hervorgerufen werden, da es einen systematischen Unterschied zwischen den vollständigen Fällen und denen mit fehlenden Werten geben könnte. Bei Verwendung des Fallausschlusses sind generell die Standardfehler größer, da in die Regression weniger Fälle eingehen.

Verwendet man das Missing-Dummy Verfahren, so ist dies ident mit einem Ersetzen durch den Mittelwert. Während Jones (1996), das Verwenden eines Missing Dummys eher skeptisch einschätzt, da es hierbei zu Verzerrungen kommen kann, schlägt Greene (2003: 60) Missing Dummys als eine mögliche Vorgehensweise beim Umgang mit fehlenden Werten vor.

Imputation hat den Vorteil, dass alle vorhandenen Daten genützt werden und Verzerrungen minimiert werden. Allerdings gibt Greene (2003: 60) zu bedenken, dass bei einfacher Imputation über die Eigenschaften des daraus entstandenen Schätzers wenig bekannt ist. Es handelt sich bei imputierten Daten nicht um echte Daten, sodass die Varianz und die Standardfehler, vor allem bei einfacher Imputation, zu klein sind (Scheffer 2002: 156). Auch beim HotdeckVerfahren können die Standardfehler unterschätzt werden. Zur Überprüfung der Güte verschiedener Imputationsmethoden werden Simulationsstudien durchgeführt. Greene zieht den Schluss, dass vor allem einfache Imputationen zu Verzerrungen führen und daher in der Regel nicht zu empfehlen sind (2003: 60). Das Ersetzen fehlender Werte durch die Mittelwerte einer Variable führt im Vergleich zum Fallausschluss zu keiner Änderung, allerdings ist das $\mathbf{R}^{\mathbf{2}}$ geringer.

Multiple Imputation schneidet in Simulationsstudien häufig besser ab, wobei das genaue Imputationsverfahren und die verwendete Statistik Software entscheidend ist (vgl. z.B. Horton, Kleinman 2007; Scheffer 2002). Im Gegensatz zu einfacher Imputation spiegelt multiple Imputation durch das wiederholte Imputieren die Unsicherheit, die durch das Imputieren entsteht, wieder. Wichtig sind die genauen Spezifikationen, da Missspezifikationen zu Verzerrungen führen können. Ein weiterer Nachteil ist, dass das Verfahren kompliziert und rechenaufwendig ist.

41 Weiterführendes ist beispielsweise in Horton und Kleinman (2007) zu finden. 
Für welche Methode man sich entscheidet, hängt auch davon $a b$, ob es sich um für das Modell wichtige Variable handelt und wie hoch der Anteil fehlender Werte ist. Generell wird empfohlen, verschiedene Varianten zu probieren und die Ergebnisse zu vergleichen, um Aussagen über die Robustheit der Ergebnisse treffen zu können.

\subsubsection{Fehlende Werte im Datensatz}

Auch die für die quantitativen Analysen verwendeten „NPO-Steuer Daten 2006“ dieser Arbeit weisen fehlende Daten auf, die darauf beruhen, dass einzelne Fragen im Fragebogen seitens der Organisationen nicht beantwortet wurden. Tabelle 8 stellt dar, wie viele fehlende Werte einzelne relevante Variable aufweisen.

\section{Tabelle 8: Fehlende Werte einzelner Variable}

\begin{tabular}{lccc} 
Variable & $\mathbf{N}$ & vorhandene Fälle in \% & fehlende Werte in \% \\
\hline vorhandene Fälle & 39613 & & \\
Anz. Ehrenamtliche & 32618 & $82,34 \%$ & $17,66 \%$ \\
Ehrenamt Dummy & 39602 & $99,97 \%$ & $0,03 \%$ \\
Kollektivvertrag & 35087 & $88,57 \%$ & $11,43 \%$ \\
Anteil Teilzeit & 39476 & $99,65 \%$ & $0,35 \%$ \\
Anteil Geringfügige & 39476 & $99,65 \%$ & $0,35 \%$ \\
Frauenanteil & 26151 & $66,02 \%$ & $33,98 \%$ \\
Anzahl Ehrenamtl./VZÄ & 32483 & $82,00 \%$ & $18,00 \%$ \\
Spenden/VZÄ & 29762 & $75,13 \%$ & $24,87 \%$ \\
Subventionen/VZÄ & 29762 & $75,13 \%$ & $24,87 \%$ \\
log. Vollzeitäquivalente & 39476 & $99,65 \%$ & $0,35 \%$ \\
\hline
\end{tabular}

Quelle: NPO-Steuer-Daten 2006, eigene Berechnungen

Die Tabelle umfasst jene Variablen, die für verschiedene Analysen verwendet wurden und fehlende Werte aufweisen. Alle anderen, in den Berechnungen verwendeten Variablen sind ohne fehlende Werte im Datensatz vorhanden. Während einige der oben genannten Variablen, wie der Ehrenamts-Dummy, die logarithmierten Vollzeitäquivalente oder der Anteil der Teilzeitkräfte, nur wenige fehlende Werte aufweisen, umfassen Spenden und Subventionen zu 25\% fehlende Werte, der Frauenanteil gar zu 34\%. Die Art der fehlenden Werte wird als MAR (missing at random) eingestuft. Es wird davon ausgegangen, dass die fehlenden Werte nicht von ihrer Höhe selbst abhängen, dass jedoch die fehlenden Werte nicht völlig zufällig verteilt sind.

Für die Analysen wurden verschiedene Methoden im Umgang mit fehlenden Werten versucht. So wurden OLS-Regressionen und Quantilsregressionen zur 
Bestimmung der Lohnhöhe mithilfe des Fallausschlusses, der Missing Dummys und einer einfachen Imputation mithilfe des „Impute“ Befehls bei STATA 9.2.

berechnet und die Ergebnisse verglichen. Für die Imputation wurden die jeweils anderen Variablen der unten angeführten Tabelle als Regressoren verwendet. Allerdings überzeugt dieses Verfahren insofern nicht, als das $\mathbf{R}^{\mathbf{2}}$ der Imputationen teils sehr gering ist und die Güte der Imputation daher zu hinterfragen ist. Tabelle 9 stellt die Ergebnisse dieses Vergleichs dar. Dargestellt sind Koeffizienten aus drei OLS Regressionen, die als abhängige Variable das logarithmierte Tageseinkommen haben. Außer den unten angeführten Variablen, wurden weitere Kontrollvariablen in der Regression inkludiert, die an dieser Stelle jedoch aus Übersichtlichkeitsgründen nicht dargestellt werden.

Tabelle 9: Ergebnisvergleich unter Verwendung unterschiedliche Methoden im Umgang mit fehlenden Werten

\begin{tabular}{|c|c|c|c|c|c|c|}
\hline & $\begin{array}{r}\text { Falle } \\
\text { Koeffizient } \\
\end{array}$ & $\begin{array}{l}\text { sschluss } \\
\text { Robuster } \\
\text { Stand.fehler } \\
\end{array}$ & $\begin{array}{r}\text { Missin } \\
\text { Koeffizient } \\
\end{array}$ & $\begin{array}{l}\text { Dummy } \\
\text { Robuster } \\
\text { Stand.fehler }\end{array}$ & $\begin{array}{r}\text { einfache } \\
\text { Koefrizient } \\
\end{array}$ & $\begin{array}{l}\text { nputation } \\
\text { Robuster } \\
\text { Stand.fehler } \\
\end{array}$ \\
\hline $\begin{array}{l}\text { Ehrenamt } \\
\text { Dummy }\end{array}$ & $-0,047249$ & 0,0607611 & $-0,1046794$ & $0,044842^{* *}$ & $-0,1089492$ & $0,0643442 *$ \\
\hline $\begin{array}{l}\text { kein Kollektiv- } \\
\text { vertrag, } \\
\text { Orientierung }\end{array}$ & $-0,1771566$ & $0.0667918^{* * *}$ & $-0,103227$ & $0.0579006^{*}$ & $-0,161624$ & $0,0561356 * * *$ \\
\hline $\begin{array}{l}\text { kein Kollektiv- } \\
\text { vertrag }\end{array}$ & $-0,0661821$ & 0,0765966 & $-0,0943734$ & 0,0634599 & $-0,1480779$ & $0,0639726^{* *}$ \\
\hline Anteil Teilzeit & 0,0943572 & 0,0712734 & 0,051487 & 0,0385091 & 0,1207449 & $0,0491686 * *$ \\
\hline $\begin{array}{l}\text { Anteil } \\
\text { Geringfügige }\end{array}$ & $-0,066834$ & 0,0920127 & $-0,1461105$ & $0,0703201^{* *}$ & $-0,0833028$ & 0,0639824 \\
\hline Frauenanteil & $-0,073672$ & 0,1286503 & 0,0127661 & 0,058805 & $-0,1163399$ & 0,0825669 \\
\hline $\begin{array}{l}\text { Anzahl } \\
\text { Ehrenamtl_/ } \\
\text { VZ̈̈ }\end{array}$ & $-0,0004991$ & 0,0005638 & $-0,0000124$ & 0,0000105 & 0,0000192 & 0,0001629 \\
\hline Spenden/ VZÄ & 0,0000002 & 0,000000274 & 0,000000242 & 0,000000302 & 0,000000252 & 0,000000326 \\
\hline Subvent_/VZÄ & 0,00000113 & $0,000000414^{* * *}$ & 0,00000113 & $0,000000469^{* *}$ & 0,00000138 & 0,000000509 \\
\hline $\begin{array}{l}\text { log. Vollzeit- } \\
\text { äquivalente } \\
\mathbf{N}\end{array}$ & $\begin{array}{c}0,0271737 \\
12158\end{array}$ & 0,0244819 & $\begin{array}{c}0,0130282 \\
39613\end{array}$ & 0,0107651 & $\begin{array}{l}0,0049 \\
39613\end{array}$ & 0,0120082 \\
\hline
\end{tabular}

Abhängige Variable: Log. Tageseinkommen, weitere Kontrollvariablen vorhanden

Quelle: NPO-Steuer-Daten 2006, eigene Berechnungen

Es zeigt sich, dass sich die Koeffizienten, mit einer Ausnahme, die den Frauenanteil betrifft und nicht signifikant ist, in ihren Vorzeichen nicht unterscheiden und auch in der Höhe vergleichbar sind. Auch die robusten Standardfehler ähneln einander, zum Teil unterscheiden sich die Variablen in ihrer statistischen Signifikanz.

Für weiterführende Berechnungen wurden Missing Dummys gewählt. Einfache Imputation erscheint aus den oben dargestellten Gründen und der geringen $\mathrm{Re}$ - 
putation in Simulationsstudien nicht geeignet. Beim Fallausschluss würde auf sehr viele Fälle verzichtet, Verzerrungen können außerdem nicht ausgeschlossen werden, da angenommen wird, dass es sich bei den fehlenden Werten um MAR Missings handelt. Nichtsdestotrotz muss bei der Interpretation der Ergebnisse bedacht werden, dass auch das Verwenden von Missing Dummys zum Teil als kritisch eingestuft wird, da es auch hier zu Verzerrungen kommen kann. Auf multiple Imputation wurde aufgrund des sehr hohen Rechenaufwands verzichtet.

\section{$8 \quad$ Ergebnisse}

In diesem Kapitel werden die Ergebnisse der quantitativen Analysen, bei denen die eben beschriebene Lohnfunktion empirisch geschätzt wird, vorgestellt. Im Kapitel 8.1 werden zuvor deskriptive Ergebnisse aus dem Datensatz gezeigt. In den Kapiteln 8.2 und 8.3 werden die Ergebnisse der OLS- und Quantilsregression präsentiert, bevor im Kapitel 8.4 die Forschungsfragen zusammenfassend beantwortet werden.

Vor der Darstellung der Ergebnisse der Regressionsschätzungen, wird ein Hinweis zur Interpretation der Ergebnisse gegeben.

Die abhängige Variable geht, wie bereits dargestellt, in logarithmierter Form in die Schätzgleichung ein, ebenso wie die Anzahl der Vollzeitäquivalente.

Bei logarithmierten stetigen Variablen können die Koeffizienten als Elastizitäten interpretiert werden. Ein Veränderung des Regressors um $p \%$ bewirkt eine Änderung von $\hat{y}$ um $b \cdot p \%$, wobei $b$ in diesem Fall den geschätzten Koeffizienten darstellt.

Die Koeffizienten der nicht-logarithmierten stetigen Variablen können als prozentuelle Änderung der abhängigen Variable bei Zunahme der unabhängigen Variable um eine Einheit interpretiert werden.

\subsection{Beschreibung der Stichprobe und deskriptive Ergebnisse}

Dieser Teil soll kurz die aus Plausibilitätsgründen reduzierte Stichprobe näher beschreiben, sodass ein besseres Verständnis dafür gewonnen wird, welche Art von Organisationen der Datensatz der weiterführenden Analysen beinhaltet.

Befragt wurden NPO verschiedener Tätigkeitsbereiche. Die folgende Tabelle gibt eine Übersicht über die Tätigkeitsbereiche der teilnehmenden Organisationen nach ÖNACE sowie die Anzahl der Organisationen. 
Tabelle 10: Anzahl der Organisationen im Datensatz nach

Tätigkeitsbereich

\begin{tabular}{|c|c|c|}
\hline $\begin{array}{l}\text { ÖNACE } \\
\text { (6-stellig) }\end{array}$ & Sektorenbezeichnung & $\begin{array}{l}\text { Anzahl } \\
\text { Organisa- } \\
\text { tionen }\end{array}$ \\
\hline 552302 & Beherbergungswesen a.n.g. & 8 \\
\hline 553002 & Buffets und Imbissstuben & 2 \\
\hline 731000 & $\begin{array}{l}\text { Forschung und Entwicklung im Bereich Natur-, Ingenieur-, } \\
\text { Agrarwissenschaften und Medizin }\end{array}$ & 5 \\
\hline 732000 & $\begin{array}{l}\text { Forschung und Entwicklung im Bereich Rechts-, Wirtschafts- und } \\
\text { Sozialwissenschaften sowie im Bereich Sprach-, Kultur- und } \\
\text { Kunstwissenschaften }\end{array}$ & 6 \\
\hline 741100 & Rechtsberatung & 1 \\
\hline 742001 & Architekturbüros & 1 \\
\hline 743000 & Technische, physikalische und chemische Untersuchung & 1 \\
\hline 801001 & Kindergärten & 3 \\
\hline 801002 & Volksschulen & 1 \\
\hline 802202 & Berufsbildende mittlere Schulen & 1 \\
\hline 803000 & Hochschulen und hochschulverwandte Lehranstalten & 2 \\
\hline 804200 & Erwachsenenbildung und Unterricht a.n.g. & 17 \\
\hline 851100 & Krankenhäuser & 1 \\
\hline 851301 & Zahnambulatorien & 1 \\
\hline 851401 & Krankentransporte und Rettungsdienste & 5 \\
\hline 851402 & Hauskrankenpflege & 5 \\
\hline 851403 & Psychotherapeuten, Klinische und Gesundheitspsychologen & 3 \\
\hline 851404 & Sonstiges Gesundheitswesen a.n.g. & 4 \\
\hline 853101 & Altenheime & 17 \\
\hline 853102 & Sonstige Heime (ohne Fremden-, Erholungs- und Ferienheime) & 12 \\
\hline 853200 & Sozialwesen a.n.g. & 104 \\
\hline 911200 & Berufsorganisationen & 12 \\
\hline 913100 & Kirchliche und sonstige religiöse Vereinigungen & 7 \\
\hline 913300 & Sonstige Interessenvertretungen und Vereine a.n.g. & 162 \\
\hline 923100 & Künstlerische und schriftstellerische Tätigkeiten und Darbietungen & 2 \\
\hline 923200 & $\begin{array}{l}\text { Betrieb und technische Hilfsdienste für kulturelle und unterhaltende } \\
\text { Leistungen }\end{array}$ & 2 \\
\hline 925100 & Bibliotheken und Archive & 3 \\
\hline 925200 & Museen und Denkmalschutz & 12 \\
\hline 925300 & Botanische und zoologische Gärten sowie Naturparks & 1 \\
\hline 926102 & Betrieb von sonstigen Sportanlagen & 1 \\
\hline 926200 & Erbringung von sonstigen Dienstleistungen des Sports & 19 \\
\hline Summe & & 421 \\
\hline
\end{tabular}


Wie aus Tabelle 10 leicht ersichtlich, ist ein Großteil der Organisationen der ÖNACE-Kategorie 913300 „Sonstige Interessenvertretungen und Vereine a.n.g“ zuzurechnen. Dies ist darauf zurückzuführen, dass diese Kategorie als eine Art „Restkategorie“ zu sehen ist, denen alle NPO zugerechnet werden, für die es keine passendere ÖNACE-Klassifizierung gibt. Viele Vereine fallen darunter. Die zweitgrößte Kategorie ist die Kategorie 853200 „Sozialwesen a.n.g. ${ }^{42 ،}$. Auch diese Kategorie kann als ,Restkategorie' bezeichnet werden, die einen Großteil der sozialen Dienste beinhaltet.

Folgende Tabelle 11 gibt einen Überblick über die Zusammensetzung der Stichprobe nach Bundesländern:

Tabelle 11: Anzahl der Organisationen im Datensatz nach Bundesland

\begin{tabular}{lc}
\multicolumn{1}{c}{ Bundesländer } & $\begin{array}{c}\text { Anzahl Org- } \\
\text { anisationen }\end{array}$ \\
\hline Wien & 135 \\
\hline Niederösterreich & 51 \\
\hline Burgenland & 19 \\
\hline Steiermark & 42 \\
\hline Kärnten & 16 \\
\hline Oberösterreich & 60 \\
\hline Salzburg & 39 \\
\hline Tirol & 33 \\
\hline Vorarlberg & 26 \\
\hline $\begin{array}{l}\text { Summe } \\
\text { Quelle: NPO-Steuer-Daten }\end{array}$ \\
eigene Berechnungen
\end{tabular}

Ein Großteil der Organisationen hat ihren Hauptsitz in Wien. Besteht eine Organisation aus mehreren Niederlassungen, wurde in diesen Fällen die Niederlassung der Zentrale gewertet.

Tabelle 12 gibt schließlich einen Überblick über die Struktur der Beschäftigten in den an der Befragung teilnehmenden Organisationen. Hier ist zu beachten, dass die Angaben aus dem Organisationsfragebogen stammen.

42 Die Abkürzung a.n.g. steht für ,anderweitig nicht genannt“. 
Tabelle 12: Struktur der Beschäftigten im Datensatz

\begin{tabular}{|c|c|c|c|}
\hline & $\begin{array}{l}\text { Mittelwert } \\
\text { (Individuen) }\end{array}$ & $\begin{array}{l}\text { Standardab- } \\
\text { weichung } \\
\text { (Individuen) }\end{array}$ & $\mathbf{N}$ \\
\hline Anzahl Ehrenamtliche & 11795,65 & 14602,41 & 32618 \\
\hline $\begin{array}{l}\text { Anteil } \\
\text { Teilzeitbeschäftigter/VZÄ } \\
\text { Anzahl Geringfügig }\end{array}$ & 0,783264 & 0,3952224 & 39613 \\
\hline Beschäftigter/VZ̈̈ & 0,057266 & 0,324103 & 39613 \\
\hline Frauenanteil/VZ̈̈ & 0,6451924 & 0,6065196 & 39613 \\
\hline Vollzeitäquivalente & 3275,317 & 2723,249 & 39613 \\
\hline
\end{tabular}

Quelle: NPO-Steuer-Daten 2006, eigene Berechnungen

Folgende Tabelle 13 zeigt deskriptive Statistiken für die Lohnvariable und inkludiert Jahresbruttolohn, Bruttotageslohn ${ }^{43}$ und den logarithmierten Bruttotageslohn.

Tabelle 13: Deskriptive Statistik für die Lohnvariable

\begin{tabular}{l|r|r|r} 
Variable & \multicolumn{1}{c}{ N } & \multicolumn{1}{c}{ Mittelwert } & \multicolumn{1}{c}{$\begin{array}{c}\text { Standard- } \\
\text { abweichung }\end{array}$} \\
\hline Bruttojahreslohn & 39613 & 15490,04 & 12049,49 \\
Bruttotageslohn & 39613 & 51,45403 & 29,97147 \\
log. Bruttotageslohn & 39613 & 3,76441 & 0,6396704
\end{tabular}

Quelle: NPO-Steuer-Daten 2006, eigene Berechnungen, Angaben in Euro

Im Mittel hatten die Individuen im Datensatz ein Bruttotageseinkommen von rund 51,5 Euro mit einer Standardabeichung von 29,97 Euro.

Abschließende Tabelle 14 zeigt die in die Regressionsmodelle eingehenden Variablen samt Referenzkategorien.

43 Der Bruttotageslohn errechnet sich aus dem Bruttojahreslohn durch die Anzahl der Bezugstage (1-365 Tage). 


\section{Tabelle 14: Beschreibung der Regressoren}

\begin{tabular}{|c|c|}
\hline Variablenname & Beschreibung \\
\hline Alter & Alter in Jahren \\
\hline Alter quadriert & Alter in Jahren quadriert \\
\hline weiblich & Dummy Variable, 1 wenn weiblich, sonst 0 \\
\hline Angestellte/r & Dummy Variable, 1 wenn Angestellte/r, sonst 0; \\
\hline Angesteinte/r & Referenzgruppe: Arbeiter/in \\
\hline Lehrling & Dummy Variable, 1 wenn Lehrling, sonst 0 \\
\hline Vertragsbediensteter & Dummy Variable, 1 wenn Vertragsbedienstete/r, sonst 0 \\
\hline Vollzeitdummy & Dummy Variable, 1 wenn Vollzeit, sonst 0 \\
\hline Ehrenamts-Dummy & Dummy Variable, 1 wenn NPO Ehrenamtliche hat, sonst 0 \\
\hline Anz.Ehrenamt//VZ̈̈ & Ehrenamtliche pro Vollzeitäquivalent \\
\hline Ehrenamts-Missing-Dummy & Dummy Variable, 1 wenn Antworten fehlen, sonst 0 \\
\hline Spenden/VZÄ & Spenden (inkl. Sponsoring) pro Vollzeitäquivalent \\
\hline Subventionen/VZÄ & $\begin{array}{l}\text { Subventionen (inkl. Einkommen aus Leistungsverträgen) pro } \\
\text { Vollzeitäquivalent }\end{array}$ \\
\hline $\begin{array}{l}\text { Missing Dummy: Spenden, } \\
\text { Subventionen }\end{array}$ & Dummy Variable, 1 wenn Antworten fehlen, sonst 0 \\
\hline Sektoren-Dummys (32) & $\begin{array}{l}\text { Dummy Variables für } 32 \text { ÖNACE Kategorien, } \\
\text { Referenzgruppe: Sozialwesen a.n.g. }\end{array}$ \\
\hline Kollektivvertrag & $\begin{array}{l}\text { Dummy Variable, I wenn Organisation Kollektivvertrag } \\
\text { unterliegt, sonst } 0 \text {, Referenzgruppe }\end{array}$ \\
\hline $\begin{array}{l}\text { Kein Kollektivvertrag, aber } \\
\text { Orientierung }\end{array}$ & $\begin{array}{l}\text { Dummy Variable, I wenn Organisation keinem Kollektivvertrag } \\
\text { unterliegt, sich jedoch daran orientiert, sonst } 0\end{array}$ \\
\hline Kein Kollektivvertrag & $\begin{array}{l}\text { Dummy Variable, } 1 \text { wenn Organisation keinem Kollektivvertrag } \\
\text { unterliegt, sonst } 0\end{array}$ \\
\hline Kollektivvertrag Missing Dummy & $\begin{array}{l}\text { Dummy Variable, } 1 \text { wenn Fragen zu Kollektivvertrag fehlen, } \\
\text { sonst } 0\end{array}$ \\
\hline Teilzeitbesch./VZÄ & $\begin{array}{l}\text { Anteil der Teilzeitbeschäftigten (Einkommen über } € 323,46 \\
\text { monatlich) innerhalb der Organisation }\end{array}$ \\
\hline Geringfügige/VZÄ & $\begin{array}{l}\text { Anteil der geringfügig Beschäftigten (Einkommen unter } \\
€ 323,46 \text { monatlich) innerhalb der Organisation }\end{array}$ \\
\hline $\begin{array}{c}\text { Frauen } / V Z \ddot{A} \\
\text { log. VZÄ }\end{array}$ & $\begin{array}{l}\text { Frauenanteil in der Organisation } \\
\text { log. Vollzeitäquivalente in einer Organisation }\end{array}$ \\
\hline Bundeslands-Dummys & Dummy Variablen für 9 Bundesländer, Referenzgruppe: Wien \\
\hline
\end{tabular}

Quelle: eigene Darstellung

Wie schon in Kapitel 6.3.1 dargestellt, geht als Regressand der logarithmierte Bruttotageslohn ein. Die Variable "Spenden“ enthält sowohl Einnahmen aus Spenden als auch aus Sponsoring, während die Variable „Subventionen“ Einnahmen aus öffentlichen Subventionen als auch aus Leistungsverträgen mit der öffentlichen Hand enthält. Der Grund dafür ist, dass die Grenzen zwischen den beiden Vertragsarten oft fließend sind (vgl. Zauner et al. 2006). Beim Alter der Individuen werden die Variablen, Alter' und ,Alter quadriert' verwendet, da ein nicht-linearer Verlauf des Zusammenhangs vermutet wird. Das Alter einer Per- 
son und das quadrierte Alter gehen in nicht-logarithmierter Form in die Schätzgleichung ein. Der Grund dafür besteht in der Annahme, dass das Alter in keiner streng linearen Beziehung zum logarithmierten Lohn steht, sondern dass der Einfluss eines zusätzlichen Lebensjahrs mit der Zeit abnimmt. Die restlichen Variablen sind entweder Dummy-Variablen oder stellen Prozentwerte dar. Zur letzteren Gruppe gehören der Anteil der geringfügig Beschäftigten, Anteil der Teilzeitkräfte und Frauenanteil. Die Anzahl der ehrenamtlichen Mitarbeiter/innen in einer Organisation, sowie die Höhe der Spenden und Subventionen wurden jeweils durch die Anzahl der Vollzeitäquivalente dividiert. Damit soll ein möglicher Größeneffekt aus den Variablen genommen werden und Multikollinearität mit der Variable „log. VZ̈̈“ verhindert werden. Die Größe der Organisation soll nicht durch die Variablen „Ehrenamt“, „Spenden“ und „Subventionen“, sondern alleinig durch die Anzahl der Vollzeitäquivalente abgebildet werden. Die Vollzeitäquivalente wurden ebenfalls logarithmiert.

\subsection{Kleinste-Quadrat-Schätzung (OLS-Regression)}

Die Schätzung der OLS-Regression erfolgt mit robusten Standardfehlern ${ }^{44}$ nach Huber-White (vgl. White 1980) ${ }^{45}$. Der Grund ist, wie weiter oben dargelegt, die besondere hierarchische Struktur der LEE-Daten. Die Residuen innerhalb einer Organisation sind nicht unabhängig voneinander, weshalb die Standardfehler dafür korrigiert werden müssen. Die Berechnung der robusten Standardfehler nach Huber-White erfolgt mithilfe der cluster-Option in STATA 9.2.

Die Ergebnisse der Schätzung werden in Folge präsentiert, wobei zwei verschiedene Modelle vorgestellt werden. Das erste (verkürzte) Modell beinhaltet Individualvariable und die nonprofit-spezifischen Organisationsvariablen, die die Forschungsfrage beantworten sollen, als exogene Variable, das zweite (erweiterte) Modell zusätzlich eine Reihe von kontrollierenden Organisationsvariablen, die auch in anderen Lohnfunktionen Standard sind. Die Verwendung unterschiedlicher Kontrollvariablen soll die Robustheit der Ergebnisse überprüfen.

Tabelle 15 stellt die Ergebnisse der beiden Modelle dar, wobei im linken Modell eine Reihe an Kontrollvariablen auf Organisationsebene fehlen. ${ }^{46}$

Vgl. dazu auch Kapitel 7.2.

45 Für diese Art von Standardfehlern sind verschiedene Namen gebräuchlich, wie: „Huber-“, „White-“ oder „Eicker-“Standardfehler, bzw. eine Kombination der Namen (vgl. Wooldridge 2002: 57).

46 Siehe dazu folgende Tabelle 16. 


\section{Tabelle 15: OLS-Regressionen, ausgewählte Ergebnisse}

abhängige Variable: log. Bruttotageseinkommen

$\mathrm{N}=39613$, Clusteranzahl: 421

\begin{tabular}{|c|c|c|c|c|}
\hline & \multicolumn{2}{|c|}{ verkürztes Modell } & \multicolumn{2}{|c|}{ ausführliches Modell } \\
\hline Modellvariable & Koeffizient & $\begin{array}{c}\text { Robuste } \\
\text { Standard-fehler }\end{array}$ & Koeffizient & $\begin{array}{c}\text { Robuste } \\
\text { Standard- } \\
\text { fehler }\end{array}$ \\
\hline \multicolumn{5}{|c|}{ Individualvariable } \\
\hline Alter & $0,05353 * * *$ & 0,0079567 & $0,0515657^{* * *}$ & $(0,0061229)$ \\
\hline Alter quadriert & $-0,0005421^{* * *}$ & 0,000095 & $-0,0005328 * * *$ & $(0,0000766)$ \\
\hline weiblich & $-0,0591666^{*}$ & 0,0304828 & $-0,0704764^{* *}$ & $(0,0315922)$ \\
\hline Lehrling & $-0,285063 * * *$ & 0,0526568 & $-0,3542278 * * *$ & $(0,0717919)$ \\
\hline Angestellte/r & $0,3779354 * * *$ & 0,0779701 & $0,4163256 * * *$ & $(0,067687)$ \\
\hline Vertragsbedienst. & $0,2462444 * *$ & 0,0994971 & $0,2950531^{* *}$ & $(0,1255232)$ \\
\hline Vollzeit & $0,6551716^{* * *}$ & 0,0280551 & $0,6420037^{* * *}$ & $(0,0212326)$ \\
\hline \multicolumn{5}{|c|}{ Organisationsvariable } \\
\hline Ehrenamt Dummy & 0,0077173 & 0,0760284 & $-0,1046794^{* *}$ & $(0,044842)$ \\
\hline $\begin{array}{c}\text { Anzahl } \\
\text { Ehrenamtliche/VZÄ }\end{array}$ & $-0,0000462 * *$ & 0,0000182 & $-0,0000124$ & $(0,0000105)$ \\
\hline Spenden/VZÄ & $-0,000000774$ & 0,000000744 & 0,000000242 & $(0,000000302)$ \\
\hline Subventionen/VZÄ & 0,000000109 & 0,000000519 & $0,00000113^{* *}$ & $(0,000000469)$ \\
\hline Konstante & $2,066608^{* * *}$ & 0,1486870 & $2,006894 * * *$ & $(0,1577107)$ \\
\hline $\begin{array}{l}\text { Missing Dummys } \\
\text { vorhanden }\end{array}$ & \multicolumn{2}{|r|}{ ja } & \multicolumn{2}{|c|}{ ja } \\
\hline $\begin{array}{c}\text { Kontrollvariable } \\
\qquad \mathbf{R}^{2}\end{array}$ & \multicolumn{2}{|c|}{$\begin{array}{c}\text { nein } \\
0.3657\end{array}$} & \multicolumn{2}{|c|}{$\underset{0.4035}{\text { ja }}$} \\
\hline
\end{tabular}

*** (**) [*] auf 99\%- (95\%-) [90\%-] Level signifikant

Quelle: NPO-Steuer-Daten 2006, eigene Berechnungen

Im Vergleich des ausführlichen Modells mit dem verkürzten Modell fällt auf, dass sich die Individualvariablen nur wenig voneinander unterscheiden, was ein Zeichen für die relative Robustheit dieser Ergebnisse ist. Die Organisationsvariable hingegen, unterscheiden sich zum Teil in ihrer Höhe, im Vorzeichen und ihrer Signifikanz. Das ist ein Hinweis darauf, dass im verkürzten Modell möglicherweise Modellvariable fehlen.

Die obere Hälfte der Tabelle enthält die Individualvariablen, Alter, Alter quadriert, Geschlecht, Lehrling, Angestellte/r, Vertragsbedienstete/r und die Dummy-Variable, ob ein Individuum vollzeitbeschäftigt ist.

Alle Vorzeichen der Koeffizienten dieser Variablen entsprechen den erwarteten Vorzeichen. Die erwartete Lohnhöhe steigt mit zunehmenden Alter, allerdings wird der Lohnanstieg mit zunehmenden Alter geringer, was anhand des negativen Vorzeichens der Variable ,Alter quadriert' ersichtlich ist. Daraus ist abzuleiten, dass die Beziehung zwischen Alter und der Lohnhöhe nicht linear ist. 
Wie zu erwarten verdienen Frauen weniger als Männer. Der Lohn von Frauen ist zwischen 6 und 7\% geringer als der der Männer. Lehrlinge verdienen weniger als Arbeiter/-innen, die die Referenzkategorie darstellen, Angestellte und Vertragsbedienstete hingegen mehr.

Wenig überraschend ist der erwartete Bruttotageslohn für die Individuen, die Vollzeit arbeiten. So erhöht sich der Lohn für Vollzeitkräfte um rund 65\%.

Die untere Hälfte der Tabelle 15 enthält nonprofit-spezifische Organisationsvariable, die der Beantwortung der Forschungsfrage dienen, also die Variablen, die Formen von (freiwilligen) Zuwendungen beinhalten. Hier ist zu sehen, dass die Ergebnisse größtenteils nicht statistisch signifikant sind. Während im verkürzten Modell nur die Anzahl der Ehrenamtlichen signifikant ist, sind im längeren Modell der Ehrenamt-Dummy und die Subventionen, nicht aber die SpendenVariable und das Verhältnis der ehrenamtlichen Mitarbeiter/-innen zu den Vollzeitäquivalenten signifikant. Im verkürzten Modell nimmt mit höherer Anzahl der Ehrenamtlichen im Verhältnis zu den Vollzeitäquivalenten der Lohn der bezahlten Beschäftigten ab. Der Lohn der bezahlten Mitarbeiter/-innen nimmt bei einer zusätzlichen ehrenamtlichen Arbeitskraft im Verhältnis zu den Vollzeitäquivalenten um ca. $0,005 \%$ ab. Im Modell mit mehr Kontrollvariablen ist der Ehrenamts-Dummy signifikant negativ. Die NPO, die freiwillige Mitarbeiter/innen haben, bezahlen um ca. 10\% weniger als die Organisationen, die keine ehrenamtlichen Mitarbeiter/-innen haben. Zusätzliche Subventionen/ Vollzeitäquivalent erhöhen den Lohn. Jeder zusätzliche Euro je Vollzeitäquivalent an Subventionen, der an die Organisationen geht, erhöht den Lohn der bezahlten Arbeitskräfte um $0,0001 \%$. Dieser Betrag mag nicht sehr hoch erscheinen, berechnet man jedoch den Beta-Koeffizienten, der auch als standardisierter Regressionskoeffizient bezeichnet wird, scheint der Betrag schon größer. Bei einem Anstieg der Subventionen pro Vollzeitäquivalent um eine Standardabweichung (ca. 32.300€), steigen die Löhne um ca. 3,6\%.

Wegen der ausführlicheren Kontrolle nach Organisationsgröße, der Zusammensetzung der Belegschaft und nach Tätigkeit der NPO, sind die Ergebnisse des Modells mit der größeren Anzahl an Kontrollvariable als verlässlicher einzuschätzen. Beim verkürzten Modell kann nicht ausgeschlossen werden, dass hinter den Variablen liegenden Faktoren auf die Ergebnisse wirken.

Zusätzlich werden in Folge auch die Koeffizienten der übrigen Kontrollvariablen dargestellt. Im zweiten (ausführlicheren) Modell werden eine Reihe zusätzlicher Organisationsvariablen, nach denen kontrolliert wurde und die auch in anderen empirischen Studien häufig in die Lohnfunktion aufgenommen werden, dargestellt, im ersten (verkürzten) Modell werden zusätzlich Missing-Dummys dargestellt. 


\section{Tabelle 16: Ergebnisse der Kontrollvariablen}

abhängige Variable: log. Bruttotageseinkommen $\mathrm{N}=39613$, Clusteranzahl: 421

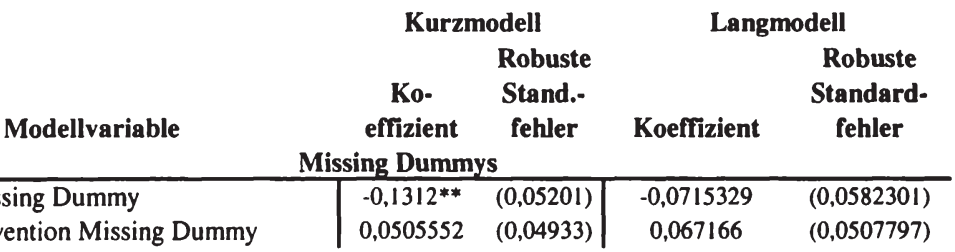

Kollektivvertrag / Zusammensetzung der Arbeitnehmer

Kein Kollektivvertrag, aber Orientierung

log. VZ̈̈

Niederösterreich

Burgenland

Steiermark

Kärnten

Oberösterreich

Salzburg

Tirol

Vorarlberg
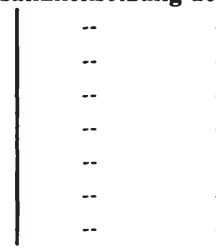

Bundesländer

-

\begin{tabular}{c|l}
-- & $-0,0437185$ \\
\hline- & $-0,0951244 *$
\end{tabular}

$-0,0951244^{*}$

0,017078

$-0,1064228$

0,0323082

$-0,1333927$

$-0,1088763$

0,0276405
$(0,0579006)$

$(0,0634599)$

$(0,0699237)$

$(0,0385091)$

$(0,0703201)$

$(0,058805)$

$(0,0107651)$

$(0,0667205)$

$(0,052885)$

$(0,0614581)$

$(0,0923848)$

$(0,0649225)$

$(0,1195829)$

$(0,0763741)$

$(0,0742718)$

*** (**) [*] auf 99\%- (95\%-) [90\%-] Level signifikant

Quelle: NPO-Steuer-Daten 2006, eigene Berechnungen

Dargestellte Variable sind Missing Dummys, die Frage, ob die Organisation einem Kollektivvertrag unterliegt oder sich in ihrem Lohnschema an einem orientiert, der Anteil der Teilzeitbeschäftigten, die nicht geringfügig angestellt sind, der Anteil der geringfügig Beschäftigten, der Frauenanteil, die Anzahl der Mitarbeiter/-innen in logarithmierter Form, Bundesländer-Dummys und ÖNACEDummys.

Bei den Missing Dummys, die angeben, ob bei den Fragen nach dem Ehrenamt und der Höhe der Spenden und Subventionen, fehlende Angaben bestehen, gibt es im verkürzten Modell ein signifikantes Ergebnis. Daraus ist zu schließen, dass das Ergebnis, dass mehr ehrenamtliche Mitarbeiter je Vollzeitäquivalent zu niedrigeren Löhnen im verkürzten Modell führen, mit Vorsicht zu interpretieren ist, da die fehlenden Werte das Ergebnis beeinflussen bzw. verzerren können. 
Robuste

Standard-

Standard-

Modellvariable

Ko-effizient fehler

Koeffizient

fehler

\section{ÖNACE Klassifikation}

\begin{tabular}{|c|c|c|c|c|}
\hline Sonstiges Beherbergungswesen a.n.g. & $\overline{--}$ & $\ddot{--}$ & 0,048158 & $(0,0870865)$ \\
\hline Restaurants und Gasthäuser & -- & -- & $-0,0124857$ & $(0,1138919)$ \\
\hline Buffets und Imbissstuben & -- & -. & $-0,4744827 * * *$ & $(0,1313139)$ \\
\hline $\begin{array}{l}\text { F\&E im Bereich Natur-, Ingenieur-, } \\
\text { Agrarwissenschaften und Medizin }\end{array}$ & -- & -. & 0,0735015 & $(0,1326757)$ \\
\hline $\begin{array}{l}\text { F\&E im Bereich Rechts-, Wirtschafts- und } \\
\text { Sozialwissenschaften sowie im Bereich Sprach-, } \\
\text { Kultur- und Kunstwissenschaften }\end{array}$ & -- & -- & $0,227134 * *$ & $(0,0996481)$ \\
\hline Rechtsberatung & -- & $-\cdot$ & $-0,2311299 * *$ & $(0,1054688)$ \\
\hline Architekturbüros & -- & -- & $0,4744472^{* * *}$ & $(0,1286225)$ \\
\hline $\begin{array}{l}\text { Technische, physikalische und chemische } \\
\text { Untersuchung }\end{array}$ & -- & $-\cdot$ & 0,1320537 & $(0,1336315)$ \\
\hline Allgemeine öffentliche Verwaltung & -- & -. & $0,1775347 * * *$ & $(0,0335189)$ \\
\hline Kindergärten & -- & -- & $-0,1403148$ & $(0,1037913)$ \\
\hline Volksschulen & -- & -- & $-0,2239386 * * *$ & $(0,0816939)$ \\
\hline Berufsbildende mittlere Schulen & -- & -- & $-0,3302296 * * *$ & $(0,014966)$ \\
\hline $\begin{array}{l}\text { Hochschulen und hochschulverwandte } \\
\text { Lehranstalten }\end{array}$ & -- & -- & $0,1763188 * *$ & $(0,0727681)$ \\
\hline Erwachsenenbildung und Unterricht a.n.g. & -- & - & 0,0724574 & $(0,0465395)$ \\
\hline Krankenhäuser & -- & $-\cdot$ & 0,1228015 & $(0,0815524)$ \\
\hline Zahnambulatorien & -- & -- & $0,3228848 * * *$ & $(0,0880584)$ \\
\hline Krankentransporte und Rettungsdienste & -- & -- & $0,2745897^{* *}$ & $(0,1271579)$ \\
\hline Hauskrankenpflege & -- & -- & $-0,0740871$ & $(0,0715429)$ \\
\hline $\begin{array}{l}\text { Psychotherapeuten, Klinische und } \\
\text { Gesundheitspsychologen }\end{array}$ & -- & -- & 0,1094711 & $(0,1416253)$ \\
\hline Sonstiges Gesundheitswesen a.n.g. & -- & -- & 0,1006002 & $(0,1126948)$ \\
\hline Altenheime & -- & -. & $0,1505082 * * *$ & $(0,054067)$ \\
\hline $\begin{array}{l}\text { Sonstige Heime (ohne Fremden-, Erholungs- und } \\
\text { Ferienheime) }\end{array}$ & -- & $-\cdot$ & $0,0840161 *$ & $(0,0472769)$ \\
\hline Berufsorganisationen & -- & $-\cdot$ & 0,0676522 & $(0,1495369)$ \\
\hline Kirchliche und sonstige religiöse Vereinigungen & -- & -- & $-0,0068617$ & $(0,1073575)$ \\
\hline $\begin{array}{l}\text { Sonstige Interessenvertretungen und Vereine } \\
\text { a.n.g. }\end{array}$ & -- & -- & 0,0142912 & $(0,0734108)$ \\
\hline $\begin{array}{l}\text { Künstlerische und schriftstellerische Tätigkeiten } \\
\text { und Darbietungen }\end{array}$ & -- & $-\cdot$ & $-0,8078553 * * *$ & $(0,1242089)$ \\
\hline $\begin{array}{l}\text { Betrieb und technische Hilfsdienste für kulturelle } \\
\text { und unterhaltende Leistungen }\end{array}$ & -- & -- & $-0,2348805 * * *$ & $(0,0791758)$ \\
\hline Bibliotheken und Archive & -- & -. & 0,0726302 & $(0,0785832)$ \\
\hline Museen und Denkmalschutz & -- & -- & $-0,0309041$ & $(0,1531597)$ \\
\hline $\begin{array}{l}\text { Botanische und zoologische Gärten sowie } \\
\text { Naturparks }\end{array}$ & -- & -- & $0,5104184 * * *$ & $(0,1284764)$ \\
\hline Betrieb von sonstigen Sportanlagen & - & -- & $0,1711383^{*}$ & $(0,0982121)$ \\
\hline $\begin{array}{l}\text { Erbringung von sonstigen Dienstleistungen des } \\
\text { Sports }\end{array}$ & -- & -- & $0,2646822 * *$ & $(0,1207688)$ \\
\hline
\end{tabular}

Sports

*** $\left.{ }^{* *}\right)\left[{ }^{*}\right]$ auf $99 \%$ - (95\%-) [90\%-] Level signifikant

Quelle: NPO-Steuer-Daten 2006, eigene Berechnungen Astrid Haider - 978-3-631-75377-4 
Individuen, die in Organisationen arbeiten, die keinem Kollektivvertrag unterliegen, sich aber an einem orientieren, verdienen weniger als solche, die einem Kollektivvertrag unterliegen. Die zweite Variable, nämlich der Dummy, dass Organisationen keinem Kollektivvertrag unterliegen, ist nicht signifikant. Diejenigen Arbeitnehmer/-innen, deren Lohnschema sich an einem Kollektivertrag orientiert, verdienen um ca. $10 \%$ weniger als jene, die in Organisationen mit Kollektivvertrag arbeiten. Allerdings ist zu beachten, dass der Missing Dummy für diese Variablen auf dem 10\%-Niveau signifikant positiv ist, was bedeutet, dass diejenigen Organisationen, die auf die Kollektivvertragsfrage die Antwort verweigerten, signifikant mehr bezahlen als diejenigen, die sie beantworteten. Das Ergebnis sollte daher mit Vorsicht interpretiert werden, da davon auszugehen ist, dass die Daten verzerrt sind.

Interessanterweise sind die Koeffizienten der Variablen, die die Beschäftigtenstruktur innerhalb der Organisation beschreiben, d.h. der Anteil geringfügig Beschäftigter, der Anteil Teilzeitbeschäftigter sowie der Frauenanteil, zumeist nicht signifikant. Nur die Variable „Anteil Geringfügig Beschäftigter“ weist ein signifikantes Ergebnis auf. Organisationen mit mehr geringfügig Beschäftigten zahlen niedrigere Löhne. Dieses Ergebnis ist keineswegs überraschend angesichts der Tatsache, dass zwischen den Teilzeitbeschäftigten, die in diesem Datensatz enthalten sind, kein Unterschied gemacht wird, wie viele Stunden sie tatsächlich arbeiten (1-34 Stunden).

Überraschenderweise weist die Organisationsgröße gemessen an den logarithmierten Vollzeitäquivalenten, kein signifikantes Ergebnis auf.

Bundeslandsvariablen wurden inkludiert, um für regionale Unterschiede beim Preis- und Lohnniveau, sowie der Arbeitsbedingungen und für unterschiedliche Arbeitslosenraten zu kontrollieren. Referenzkategorie stellt Wien dar. Hier gibt es nur ein signifikantes Ergebnis, nämlich für das Burgenland, wo niedrigere Löhne bezahlt werden als in Wien.

In die Schätzgleichung wurde eine Reihe von ÖNACE Dummys aufgenommen, wobei die Kategorie ,Sozialwesen a.n.g.' als Referenzkategorie dient. Der Grund, warum diese Kategorie als Referenzkategorie gewählt wurde, ist, dass viele NPO diese ÖNACE-Klassifizierung haben. Das Sozialwesen in Österreich zeichnet sich u.a. durch eher niedrige Löhne, einem hohen Frauenanteil und Teilzeitanteil unter Frauen aus (vgl. Trukeschitz 2006: 221).

Signifikante höhere Löhne werden in den Branchen „Forschung und Entwicklung im Bereich Rechts-, Wirtschafts- und Sozialwissenschaften sowie im Bereich Sprach-, Kultur- und Kunstwissenschaften“, „Architekturbüros“, „Allgemeine Öffentliche Verwaltung“, „Hochschulen und hochschulverwandte Lehranstalten“, „Zahnambulatorien“, „Krankentransporte und Rettungsdienste“, „Altenheime“, „Sonstige Heime“, „Botanische und zoologische Gärten sowie Naturparks“, „Betrieb von sonstigen Sportanlagen“ und „Erbringung von sonsti- 
gen Dienstleistungen des Sports“ bezahlt. Vor allem in den ersten fünf der genannten Kategorien ist davon auszugehen, dass das positive Lohndifferenzial auf der durchschnittlich höheren Qualifikation der in der Branche Beschäftigten beruht.

Signifikant niedrigere Löhne als im Bereich „Sozialwesen“ werden in den Branchen „Buffets und Imbissstuben“, „Rechtsberatung“, „Volksschulen“, „Berufsbildende mittlere Schulen“, „Künstlerische und schriftstellerische Tätigkeiten und Darbietungen“ sowie „Betrieb und technische Hilfsdienste für kulturelle und unterhaltende Leistungen“ gezahlt. Vor allem in den Bereichen „Rechtsberatung“, „Volksschulen“ und „Berufsbildenden mittleren Schulen" erscheint das Ergebnis auf den ersten Blick überraschend. Eine mögliche Erklärung für das Ergebnis könnte sein, dass z.B. im Bereich der Volksschulen nicht, wie anzunehmen wäre, vor allem Lehrer/-innengehälter in die Schätzgleichung eingingen, sondern Löhne von Angestellten in Schulvereinen (wie etwa einem Elternverein). Volksschulen als solche wurden in der Befragung nicht kontaktiert, wohl aber diverse Vereine, die im Umfeld einer Schule gegründet wurden und folglich die ÖNACE-Klassifizierung „Volksschulen“ bekommen haben. Im Fall der „Rechtsberatung“ könnte es sich um eher staatsferne, spendenfinanzierte Organisationen handeln, die Minderheiten oder benachteiligte Gruppen beraten und die durch mangelnde Zahlungsfähigkeit der Klient/-inn/en nur begrenzte Möglichkeiten besitzen, hohe Löhne zu zahlen. In diesem Fall könnte auch argumentiert werden, dass die Arbeitsspendenhypothese als besonders zutreffend gilt.

Niedrigere Löhne im Vergleich zur Kategorie „Sozialwesen a.n.g“ können folgendermaßen interpretiert werden:

- Erstens kann die Branche mit den niedrigeren Löhnen bessere unbeobachtete Arbeitsbedingungen aufweisen; oder

- zweitens: Individuen, die in dieser Branche arbeiten, sind durchschnittlich schlechter ausgebildet bzw. qualifiziert; oder

- drittens: Individuen sind eher bereit, in dieser Branche gemäß der Arbeitsspendenhypothese auf einen Teil des Einkommens zu verzichten, da die Organisation eine in ihren Augen wertvolle Tätigkeit verrichtet; oder

- viertens: Arbeitnehmer/-innen zeichnen sich in dieser Branche durch höhere intrinsische Motivation aus, sodass Effizienzlöhne einen weniger großen Stellenwert haben, oder

- fünftens: In der Branche haben Organisationen eine generell weniger sichere Finanzierungslage, sodass die Möglichkeiten, RentSharing zu betreiben sehr eingeschränkt sind. 
Für Branchen mit höheren Löhnen im Vergleich zur ÖNACE-Kategorie „Sozialwesen" gelten diese Erklärungen mit umgekehrten Vorzeichen.

Zusammenfassend lässt sich festhalten, dass im OLS-Regressionsmodell mit robusten Standardfehlern in Bezug zu den nonprofit-spezifischen Organisationsvariablen der Ehrenamts-Dummy einen signifikant negativen und die Subventionen/Vollzeitäquivalent-Variable einen signifikant positiven Koeffizienten aufweisen. Organisationen ohne ehrenamtliche Mitarbeiter/-innen zahlen Löhne, die ca. 10\% höher sind als NPO mit freiwilligen Mitarbeiter/-innen. Ein zusätzlicher Euro an Subventionen/Vollzeitäquivalent erhöhen den Lohn der bezahlten Arbeitskräfte um $0,0001 \%$. Als problemhaft bei der OLS-Schätzung kann einerseits die unbeobachtete Heterogenität der Individuen betrachtet werden, die durch fehlende Angaben zur Ausbildung und Arbeitserfahrung bestehen. Dieses Problem könnte durch eine Panelschätzung behoben werden, da dann für unbeobachtete Heterogenität der Individuen kontrolliert werden könnte. Leider ist eine derartige Schätzung aufgrund fehlender Daten nicht möglich.

Andererseits besteht auch die Frage, ob die Auswirkungen unabhängiger Variablen über die gesamte Lohnverteilung hinweg stabil sind (vgl. Hübler 2003: 551). Aus diesem Grund soll anschließend eine Quantilsregression die Zusammenhänge auf mehreren Ebenen der Lohnverteilung genauer untersuchen.

\subsection{Quantilsregression}

In diesem Teil werden die Ergebnisse der Quantilsregressionen präsentiert. Da die Quantilsschätzung als Methode eher unbekannt ist, werden zuerst das Schätzverfahren und dessen Vorteile genauer erläutert, bevor im Anschluss die Ergebnisse dargestellt werden.

\subsubsection{Schätzverfahren und Vorgehensweise}

Eine Quantilsregression ermöglicht einen genaueren Einblick in die Lohnstruktur der Arbeitnehmer/-innen im Nonprofit Sektor. Sie zeigt auf, ob und wie exogene Variable in verschiedenen Bereichen bzw. Quantilen der Lohnverteilung unterschiedlichen Einfluss auf die Lohnhöhe der Arbeitnehmer/-innen haben.

Die Methode der Quantilsregression wird zumeist auf Koenker und Bassett (1978) zurückgeführt und kann wie folgt dargestellt werden ${ }^{47}$ :

Gleich wie bei der Methode der kleinsten Quadrate (OLS) soll der Einfluss einer Reihe von unabhängigen Variablen $X_{i j}$, in diesem Fall ein Vektor, der Arbeit-

47 Für eine ausführliche Darstellung wird auf Koenker (2005) verwiesen. Für eine anwendungsorientierte Einführung siehe Buchinsky (1998). 
nehmercharakteristika sowie Organisationscharakteristika beinhaltet, auf die logarithmierte Lohnhöhe $y_{y}$ eines Arbeitnehmers bzw. einer Arbeitnehmerin $i$ in der Organisation $j$ geschätzt werden. $\theta$ stellt das $\theta-t e$ Quantil der bedingten Verteilungsfunktion von $y_{i}$ als eine lineare Funktion der unabhängigen Variablen dar (vgl. Buchinsky 1998: 94):

$$
Q_{\theta}\left(y_{i j} \mid X_{i j}\right)=X_{i j} \beta_{\theta}, \theta \in(0,1) \text {. }
$$

Sortiert man die Beobachtungen in einem Datensatz aufsteigend in zwei Teile, so ist das $\theta$-Quantil einer Verteilung ein Wert, der diese Menge teilt. Der untere Teil, d.h. der Teil mit den kleineren Werten, umfasst dabei $\theta \%$ der Werte, der andere $(1-\theta) \%$. Der Median ist ein Spezialfall mit $\theta=0.5$, d.h. $50 \%$ der Organisationen haben kleinere Beobachtungen, $50 \%$ größere.

Der Schätzer des Modells der Quantilsregression lautet nach Lösung des Minimierungsproblems wie folgt (Koenker, Bassett 1978: 38):

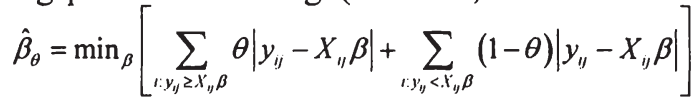

wobei $0<\theta<1$. Vereinfacht kann das Minimierungsproblem auch als

$$
\hat{\beta}_{\theta}=\min _{\beta} \sum_{1} \rho_{\theta}\left(y_{i j}-X_{i j} \beta\right)
$$

dargestellt werden. $\rho_{\theta}$ wird dabei als check function bezeichnet, die positiven Residuen $u$ das Gewicht $\theta$ zuweist, negativen $(1-\theta)$ :

$$
\rho_{\theta}(u)=[\theta-I(z<0)] u=\left\{\begin{array}{l}
|u|=\theta u \\
(1-\theta)|u|=(\theta-1) u
\end{array} \quad \text { für } \begin{array}{l}
u \geq 0 \\
u<0
\end{array}\right.
$$

Der Schätzer $\hat{\beta}_{\theta}$ ist konsistent und asymptotisch normalverteilt, allerdings ist er im Unterschied zum OLS-Schätzer nicht effizient (vgl. Buchinsky 1998: 95 und 98).

Interpretiert werden können die einzelnen Koeffizienten als die marginale Änderung des $\theta$-ten Quantils der bedingten Verteilungsfunktion durch die marginale Änderung der relevanten unabhängigen Variable. Allerdings ist zu beachten, dass eine Person im $\theta$-ten Quantil einer bedingten Verteilung durch Änderung der $X$ in einem anderen Quantil zu finden sein kann (vgl. Buchinsky 1998: 98).

Durch Variieren von $\theta$ ist es möglich, Ergebnisse für die gesamte Verteilung der Verteilungsfunktion der abhängigen Variable zu erhalten. Die ist vor allem dann vorteilhaft, wenn diese nicht homoskedast ist, da im Unterschied zur OLS Schätzung nicht nur der bedingte Mittelwert, sondern mehrere Werte auf der Lohnverteilung geschätzt werden. 
Während man bei der OLS-Regression davon ausgeht, dass die bedingte Verteilung der abhängigen Variable, in diesem Fall also der logarithmierten Lohnhöhe, bei einer Reihe gegebener erklärender Variablen homogen ist, lässt die Quantilsregression Heterogenität zu (vgl. z.B. Wagner et al. 2006: 507). Quantilsregression ist vor allem dann sinnvoll, wenn anzunehmen ist, dass die bedingte Verteilung der abhängigen Variable nicht einfach durch deren Mittelwert und deren Varianz charakterisiert werden kann. Die OLS-Regression geht davon aus, dass die Beziehung zwischen abhängiger und unabhängiger Variable an allen Punkten der Verteilung des Regressanden dieselbe ist. Die Quantilsregression hingegen will herausfinden, ob die unabhängige Variable an unterschiedlichen Punkten der bedingten Verteilung der abhängigen Variable unterschiedlichen Einfluss hat. So kann sich beispielsweise die Einführung eines Kollektivvertrags für niedrige Einkommen anders auswirken als für hohe Löhne, da im Allgemeinen angenommen wird, dass Kollektivverträge die Lohnspreizung zwischen niedrigen und hohen Einkommen reduziert. ${ }^{48}$ Demnach würde der Koeffizient eines Kollektivvertrags-Dummys für das untere Einkommensquantil höher sein als der der höheren Lohnquantile.

Im Unterschied zur OLS-Schätzung werden nicht die Quadrate, sondern die absoluten Werte der Residuen minimiert. Bei der Quantilsregression wird beispielsweise die bedingte Medianfunktion anhand der Minimierung der Summe der absoluten Fehler geschätzt. Alle anderen Quantile werden anhand der Minimierung einer asymmetrisch gewichteten Summe der absoluten Fehler geschätzt. Zu beachten ist, dass die Ergebnisse der Quantilsregression nicht dieselben sind als wenn man auf ein Subset von Daten, d.h. verschiedene Perzentile der endogenen Variable, die OLS-Regression anwendet (vgl. Wagner et al. 2006: 507). Bei der Quantilsregression werden für alle Teilschätzungen alle Daten verwendet, wobei jedoch manche Beobachtungen ein höheres Gewicht bekommen als andere. Bei Vorliegen von Heteroskedastie bzw. von Nicht-Normalverteilung der abhängigen Variable ist die Quantils-regression also vorteilhafter als die Methode der kleinsten Quadrate. Wenn die Fehlerterme nicht normalverteilt sind, wobei eine Normalverteilung der Fehlerterme als eine Voraussetzung der OLS-Regression gilt, kann die Quantilsregression eine bessere Schätzung erreichen und ist damit weniger ausreißerempfindlich (vgl. z.B. Schulze 2004: 51). Der Grund dafür ist, dass anders als bei der OLS-Schätzung nicht die Quadrate der Residuen minimiert werden, sondern die absoluten Abweichungen.

Bei der Berechnung der Schätzwerte ist es, anders als bei einer OLS Regression, nicht möglich, die Ableitung der Zielfunktion zu bilden und das daraus entstandene Normalgleichungssystem nach den Schätzwerten zu lösen. Deshalb wird es als lineares Optimierungsproblem gelöst (vgl. z.B. Schulze 2004: 24ff.). 
Der Rechenaufwand zur Lösung des Minimierungsproblems kann vor allem bei großen Datensätzen sehr hoch sein. Dies kann auch darauf zurückgeführt werden, dass zumeist zur Berechnung der Standardfehler Bootstrapping ${ }^{49}$ verwendet wird. Mittels Bootstrapping werden Standardfehler und folglich Konfidenzintervalle und $p$-Werte für die Teststatistiken errechnet. Der Grund dafür liegt darin, dass unter Annahme heteroskedastisch verteilter Residuen verzerrte Standardfehler berechnet werden, sodass Bootstrapping zur Berechnung der Standardfehler vorzuziehen ist (vgl. Gould 1992, Gould 1997).

Die Methode der Quantilsregression erfreut sich, aufgrund der oben genannten Vorteile, in der angewandten Literatur zunehmender Beliebtheit. Dies zeigt die Fülle an neueren Beiträgen, in denen die Methode der Quantilsregression verwendet wird. Neuere Beiträge, in denen Löhne mittels Quantilsregression analysiert werden, sind z.B. Andini (2007), Choi, Jeong (2007), Kahn (1998), Machado, Mata (2005) und Melly (2005). Für den Bereich der Löhne im Nonprofit Sektor wurde die Quantilsregression noch nicht angewandt.

\subsubsection{Ergebnisse und Diskussion}

Im Folgenden werden die Ergebnisse der Quantilsregression dargestellt und diskutiert. Geschätzt wurde die Quantilsregression simultan für fünf Quantile $\theta=0,1, \theta=0,25, \theta=0,5, \theta=0,75$ und $\theta=0,9$. Die Schätzung erfolgt mittels Bootstrapping. Die Standardfehler sind dabei nicht exakt bestimmbar, da sie anhand einer zufälligen Stichprobe, die aus den Daten gezogen wird, berechnet werden. Die Anzahl der Replikationen beim Bootstrapping beträgt 100. Aufgrund der hohen Rechendauer, die sich bei großen Fallzahlen bei diesem Verfahren ergibt, wurde auf Berechnungen, die auf mehr Wiederholungen basieren, verzichtet. Für die Anzahl der Wiederholungen zur Berechnung der Standardfehler gibt es keine generelle Regel. Theoretisch müsste eine infinite Anzahl an Replikationen durchgeführt werden, da die Ergebnisse jedoch relativ schnell konvergieren, ist eine endliche Anzahl ausreichend. Die Wahl von 100 Replikationen wurde jedoch willkürlich gewählt. Geschätzt wurden die Quantilsregressionen mithilfe des Programms STATA 9.2.

Das verwendete Modell bzw. die geschätzte Lohnfunktion ist dieselbe als in den vorherigen Kapiteln. Dennoch sollten die Ergebnisse der OLS-Regression nicht direkt mit den Ergebnissen der Quantilsregression verglichen werden, da die beiden Analysearten etwas Unterschiedliches messen.

Tabelle 17 zeigt die Ergebnisse für die Variablen, die die Forschungsfrage beantworten soll. Dargestellt sind die Koeffizienten und die Bootstrap Standardfehler in Klammern für die Lohnquantile $0,1,0,25,0,5,0,75$ und 0,9.

49 Für einen Überblick über die Methode des „Bootstrapping“ siehe z.B. Horowitz (2001) und Efron, Tibshirani (1993). 
Tabelle 17: Ausgewählte Ergebnisse der Quantilsregression

abhängige Variable: log. Bruttotageseinkommen

N=39613; Anzahl der Cluster: 421
0,1
0,25
0,5
0,75
0,9

\begin{tabular}{|c|c|c|c|c|c|}
\hline Variable & \multicolumn{5}{|c|}{ Koeffizient (Bootstrap Standardfehler) } \\
\hline $\begin{array}{c}\text { Ehrenamt } \\
\text { Dummy }\end{array}$ & $\begin{array}{c}-0,0740044 * * * \\
(0,0151079)\end{array}$ & $\begin{array}{c}-0,0930629 * * * \\
(0,0130728)\end{array}$ & $\begin{array}{c}-0,1069088 * * * \\
(0,0118074)\end{array}$ & $\begin{array}{c}-0,1008387^{* * *} \\
(0,0120302)\end{array}$ & $\begin{array}{c}-0,0671784 * * * \\
(0,013088)\end{array}$ \\
\hline $\begin{array}{c}\text { Anz. } \\
\text { Ehren- } \\
\text { amtliche } \\
\text { /VZ̈̈ }\end{array}$ & $\begin{array}{c}0,000032 \\
(0,0001144)\end{array}$ & $\begin{array}{c}-0,0000147 \\
(0,0001444)\end{array}$ & $\begin{array}{l}-0,00000946 \\
(0,0002351)\end{array}$ & $\begin{array}{c}-0,0000256 \\
(0,0000263)\end{array}$ & $\begin{array}{c}-0,0000503 \\
(0,0000921)\end{array}$ \\
\hline $\begin{array}{l}\text { Spenden } \\
\text { NVZ̈̈ }\end{array}$ & $\begin{array}{c}-0,0000012 \\
(0,000000766)\end{array}$ & $\begin{array}{c}-0,000000677 \\
(0,000000929)\end{array}$ & $\begin{array}{c}0,000000268 \\
(0,000000249)\end{array}$ & $\begin{array}{c}0,000000395^{* *} \\
(0,0000002)\end{array}$ & $\begin{array}{c}0,000000635 \\
* * * \\
(0,000000247)\end{array}$ \\
\hline \multirow{2}{*}{$\begin{array}{c}\text { Sub- } \\
\text { ventionen } \\
\text { /VZ̈̈ }\end{array}$} & $\begin{array}{c}0,000000708 * * \\
(0,000000292)\end{array}$ & $\begin{array}{c}0,00000105^{* * *} \\
(0,000000208)\end{array}$ & $\begin{array}{c}0,00000111 * * * \\
(0,000000238)\end{array}$ & $\begin{array}{c}0,00000138 * * * \\
(0,000000214)\end{array}$ & $\begin{array}{l}0,00000114^{* * * *} \\
(0,000000208)\end{array}$ \\
\hline & $\begin{array}{c}\text { Pseudo- } \\
\mathbf{R}^{2}=0,2949\end{array}$ & $\begin{array}{c}\text { Pseudo- } \\
\mathrm{R}^{2}=0,2494\end{array}$ & $\begin{array}{c}\text { Pseudo- } \\
R^{2}=0,2643\end{array}$ & $\begin{array}{c}\text { Pseudo- } \\
R^{2}=0,2670\end{array}$ & $\begin{array}{c}\text { Pseudo- } \\
R^{2}=0,2655\end{array}$ \\
\hline
\end{tabular}

Kontrollvariable auf Individual- und Organisationsebene inkludiert

*** (**) [*] am 99\%-(95\%-) [90\%-] Level signifikant

Quelle: NPO-Steuer-Daten 2006, eigene Berechnungen

Bei Betrachtung der Tabelle fällt auf, dass zwei Variable über alle Quantile signifikante Ergebnisse haben, nämlich der Ehrenamts-Dummy und die Höhe der Subventionen. Während die Anzahl der ehrenamtlichen Mitarbeiter/-innen pro Vollzeitäquivalent nicht signifikante Ergebnisse über alle Quantile hat, sind die Ergebnisse der Spendenvariable gemischt.

Der Koeffizient des Ehrenamts-Dummys ist negativ, d.h. der erwartete Lohn ist niedriger, wenn eine Organisation ehrenamtliche Mitarbeiter/-innen hat im Vergleich zu Organisationen ohne Freiwillige. Es ist jedoch nicht entscheidend, wie viele freiwillige Mitarbeiter/-innen im Vergleich zu Vollzeitäquivalenten in einer Organisation tätig sind, da die Variable „Anzahl Ehrenamtliche pro Vollzeitäquivalent" keine signifikanten Ergebnisse aufweist. Der Effekt des Ehrenamts-Dummys ist an den Rändern der Lohnverteilung schwächer ausgeprägt als in den mittleren drei Quantilen, wie an den niedrigeren Koeffizienten im 0,1 und 0,9 Quantil zu sehen ist. Die Hypothese, dass der Effekt einer Arbeitsspende besonders für die oberen Lohnklassen stark ausgeprägt sein müsste, da Manager/-innen die soziale Wirkung ihrer Organisation erkennen können, kann damit nicht bestätigt werden. Allerdings wird hier eine Aussage über den relativen und nicht dem absoluten Lohnabschlag getätigt, da die Koeffizienten als Prozente interpretiert werden können. Wenn also eine Organisation Ehrenamtliche beschäftigt, dann könnten Arbeitnehmer/-innen des 0,5 Lohnquantils 
und des 0,9 Lohnquantils z.B. den gleichen Lohnabschlag hinnehmen müssen, der Anteil des Lohnabschlags am Gesamtlohn ist jedoch für das 0,5 Lohnquantil höher als für das 0,9 Lohnquantil. Eine Erklärung für den kleineren Effekt im unteren Lohnquantil könnte sein, dass bei schon geringen Löhnen wenig Spielraum für die Organisation da ist, die Löhne noch weiter zu senken. Individuen, die in Organisationen mit ehrenamtlichen Mitarbeiter/-innen arbeiten, verdienen zwischen 6,7\% und 10,6\% weniger als jene, die in Organisationen ohne Freiwillige arbeiten. Das negative Vorzeichen der Koeffizienten zeigt, dass die Arbeitsspendenhypothese zur Erklärung des Phänomens von Relevanz sein kann. Individuen, die als Ehrenamtliche arbeiten, können ein Indikator für die soziale Erwünschtheit des Gutes der NPO sein. Wenn Freiwillige bereit sind, Zeit für diese Mission zu widmen, sind auch bezahlte Mitarbeiter/-innen bereit, auf einen Teil ihres Einkommens zu verzichten und deshalb niedrigere Löhne zu akzeptieren. Eine weitere Erklärung des negativen Vorzeichens der Koeffizienten könnte sein, dass bezahlte Mitarbeiter/-innen in NPO mit Ehrenamtlichen relativ gesehen geringere Arbeitsanforderungen haben bzw. weniger Verantwortung im Vergleich zu Individuen, die in NPO ohne freiwillige Mitarbeiter/-innen tätig sind. Leider ist es, aufgrund der Datenlage nicht möglich, genauere Angaben zur Ausbildung, Arbeitserfahrung und zu Arbeitsplatzprofilen mit einzubeziehen und danach zu kontrollieren. Weitere Erklärungen sind, dass in NPO mit ehrenamtlichen Mitarbeiter/-innen die Notwendigkeit, Effizienzlöhne zu zahlen, weniger stark vorhanden ist, da Freiwillige als Substitute gelten, oder dass bezahlten Arbeitskräfte aufgrund des Vorhandenseins freiwilliger Mitarbeiter/-innen eine weniger starke Verhandlungsmacht bei Lohnverhandlungen haben.

Spenden haben einen sehr kleinen, aber positiven Koeffizienten am oberen Ende der Lohnverteilung. Bei höheren Löhnen führt eine Zunahme an Spenden zu Lohnsteigerungen. Spenden können dahingehend interpretiert werden, dass sie Indikatoren für besondere Qualitäten der Manager/-innen sein könnten. Höhere Spenden können positiv mit höheren Fähigkeiten bzw. Qualitäten von Manager/innen korrelieren. Subventionen führen auch zu einem höheren erwarteten Lohn. Im Gegensatz zur Spendenvariable gilt dieses Ergebnis jedoch für alle Lohnquantile. Die Koeffizienten beider Variablen sind sehr klein. Für Subventionen gilt, dass ein zusätzlicher Euro zu einer Steigerung des Lohns um 0,0000708\% am 0,1 Lohnquantil und zu einer Steigerung um 0,000114\% am 0,9 Lohnquantil führt. Dieser Effekt scheint sehr klein zu sein, zieht man wiederum den Betakoeffizienten in Betrachtung, ist der Koeffizient nicht mehr ganz so klein. Werden beispielsweise öffentliche Subventionen um eine Standardabweichung erhöht (ca. 32.300€), führt dies zu einer Lohnsteigerung um 2,3\% am 0,1 Lohnquantil und um 3,7\% am 0,9 Lohnquantil. Eine Erhöhung der Spenden um eine Standardabweichung (ca. 19.500€) erhöht den Tageslohn um 0,8\% am 0,75 Lohnquantil und um 1,2\% am 0,9 Lohnquantil. In beiden Fällen profitieren die höheren Lohnklassen mehr von höheren Spenden und Subventionen. Die Resultate können mit der Rent-sharing Theorie erklärt werden. Höhere Subventionen und 
Spenden eröffnen mehr Möglichkeiten, die zusätzlichen Mittel mit der Belegschaft zu „teilen“, d.h. höhere Löhne zu bezahlen, wobei noch einmal zu betonen ist, dass der Effekt sehr klein ist. Renten werden jedoch nicht aliquot an alle Lohngruppen weitergegeben, weshalb der Effekt bei den oberen Lohnquantilen größer ist. Die Ergebnisse stimmen mit den Befunden von Preston (1988) überein, die auch einen kleinen, positiven Einfluss von Spenden und Subventionen auf die Löhne von Arbeitnehmer/-innen in NPO fand.

Die folgenden fünf Tabellen zeigen die Koeffizienten der Variablen, nach denen in der Schätzung zusätzlich kontrolliert wurde, der Übersicht halber thematisch getrennt dargestellt.

\section{Tabelle 18: Weitere Ergebnisse der Quantilsregression}

\begin{tabular}{|c|c|c|c|c|}
\hline \multicolumn{5}{|c|}{ abhängige Variable: log. Bruttotageseinkommen } \\
\hline \multicolumn{5}{|c|}{ Individualvariable } \\
\hline Variable & Koef. Q10 & $\begin{array}{l}\text { Bootstr. } \\
\text { Standard- } \\
\text { fehler }\end{array}$ & Koef. Q25 & $\begin{array}{l}\text { Bootstr. } \\
\text { Standard- } \\
\text { fehler }\end{array}$ \\
\hline Alter & $0,0709634 * * *$ & $(0,0036243)$ & $0,0611012 * * *$ & $(0,0031709)$ \\
\hline Alter quadriert & $-0,000755 * * *$ & $(0,0000472)$ & $-0,0006514 * * *$ & $(0,0000381)$ \\
\hline weiblich & $-0,0245387 * *$ & $(0,0110555)$ & $-0,0467528 * * *$ & $(0,0075574)$ \\
\hline Lehrling & $-0,1949098 * * *$ & $(0,0478761)$ & $-0,2532849 * * *$ & $(0,0359928)$ \\
\hline Angestellte/r & $0,3675762 * * *$ & $(0,0101997)$ & $0,4553715^{* * *}$ & $(0,008832)$ \\
\hline Vertragsbedienstete/r & $0,6040969 * *$ & $(0,3049092)$ & $0,4493911 * *$ & $(0,225012)$ \\
\hline \multirow[t]{2}{*}{ Vollzeit } & $1,018247 * * *$ & $(0,0151079)$ & $0,7200578 * * *$ & $(0,0077195)$ \\
\hline & Koef. Q50 & $\begin{array}{l}\text { Bootstr. } \\
\text { Standard- } \\
\text { fehler }\end{array}$ & Koef. Q75 & $\begin{array}{c}\text { Bootstr. } \\
\text { Standard- } \\
\text { fehler }\end{array}$ \\
\hline Alter & $0,033018^{* * *}$ & $(0,0014261)$ & $0,0329468^{* * *}$ & $(0,0014866)$ \\
\hline Alter quadrient & $-0,0003213 * * *$ & $(0,0000179)$ & $-0,0003137^{* * *}$ & $(0,0000195)$ \\
\hline weiblich & $-0,0751558 * * *$ & $(0,0069995)$ & $-0,1216715^{* * *}$ & $(0,0075849)$ \\
\hline Lehrling & $-0,4648869 * * *$ & $(0,0460874)$ & $-0,4460233^{* * *}$ & $(0,0449167)$ \\
\hline Angestellte/r & $0,4527768^{* * *}$ & $(0,0066169)$ & $0,4569 * * *$ & $(0,0068241)$ \\
\hline Vertragsbedienstete/r & 0,3341648 & $(0,2312994)$ & $0,2753395^{*}$ & $(0,1475907)$ \\
\hline \multirow[t]{2}{*}{ Vollzeit } & $0,5434035 * * *$ & $(0,0047006)$ & $0,444844 * * *$ & $(0,0051438)$ \\
\hline & Koef. Q90 & $\begin{array}{l}\text { Bootstr. } \\
\text { Standard- } \\
\text { fehler }\end{array}$ & & \\
\hline Alter & $0,0327851^{* * *}$ & $(0,0020902)$ & & \\
\hline Alter quadriert & $-0,0002805 * * *$ & $(0,0000271)$ & & \\
\hline weiblich & $-0,1468218 * * *$ & $(0,009389)$ & & \\
\hline Lehrling & $-0,4998354 * * *$ & $(0,0402272)$ & & \\
\hline Angestellte/r & $0,4346584 * * *$ & $(0,008346)$ & & \\
\hline Vertragsbedienstete/r & $0,1395793^{*}$ & $(0,0839925)$ & & \\
\hline Vollzeit & $0,3861712 * * *$ & $(0,0064844)$ & & \\
\hline \multicolumn{5}{|c|}{ **** $\left({ }^{* *}\right)[*]$ am 99\%-(95\%-) [90\%-] Level signifikant } \\
\hline
\end{tabular}


Die Koeffizienten der Individualvariablen in der Quantilsregression entsprechen alle den erwarteten Vorzeichen. Erwähnenswert sind die Ergebnisse für den Männlich/weiblich-Dummy und den Vollzeit/Teilzeit-Dummy: Während der Lohnunterschied zwischen Frauen und Männern bei höheren Löhnen größer wird, wird er bei vollzeitbeschäftigten Arbeitnehmer/-innen im Vergleich zu teilzeitbeschäftigten bei höheren Löhnen kleiner.

\section{Tabelle 19: Weitere Ergebnisse der Quantilsregression (Forts.)}

Missing Dummys

\begin{tabular}{|c|c|c|c|c|}
\hline Variable & Koef. Q10 & $\begin{array}{c}\text { Bootstr. } \\
\text { Standard- } \\
\text { fehler }\end{array}$ & Koef. Q25 & $\begin{array}{c}\text { Bootstr. } \\
\text { Standard- } \\
\text { fehler }\end{array}$ \\
\hline Ehrenamt Missing Dummy & $-0,0001082$ & $(0,0424189)$ & $-0,0914931 * * *$ & $(0,0247463)$ \\
\hline \multirow[t]{2}{*}{$\begin{array}{l}\text { Spenden/Subvention Missing } \\
\text { Dummy }\end{array}$} & $0,1309476^{* * *}$ & $(0,0261275)$ & $0,095974 * * *$ & $(0,0176314)$ \\
\hline & Koef. Q50 & $\begin{array}{l}\text { Bootstr. } \\
\text { Standard- } \\
\text { fehler }\end{array}$ & Koef. Q75 & $\begin{array}{l}\text { Bootstr. } \\
\text { Standard- } \\
\text { fehler }\end{array}$ \\
\hline Ehrenamt Missing Dummy & $-0,0868182 * * *$ & $(0,017506)$ & $-0,0666873 * * *$ & $(0,0156751)$ \\
\hline \multirow[t]{2}{*}{$\begin{array}{l}\text { Spenden/Subvention Missing } \\
\text { Dummy }\end{array}$} & 0,0095514 & $(0,0119662)$ & $-0,0184786$ & $(0,0118556)$ \\
\hline & Koef. Q90 & $\begin{array}{c}\text { Bootstr. } \\
\text { Standard- } \\
\text { fehler }\end{array}$ & & \\
\hline Ehrenamt Missing Dummy & $-0,0070317$ & $(0,0222128)$ & & \\
\hline $\begin{array}{l}\text { Spenden/Subvention Missing } \\
\text { Dummy }\end{array}$ & 0,0052763 & $(0,0149961)$ & & \\
\hline
\end{tabular}

*** (**) [*] am 99\%-(95\%-) [90\%-] Level signifikant

Quelle: NPO-Steuer-Daten 2006, eigene Berechnungen

In Tabelle 19 sind die Missing Dummys für die Variablen Ehrenamt und Spenden/Subventionen dargestellt. Die Dummys nehmen jeweils den Wert 1 an, wenn die Frage nach der Anzahl der ehrenamtlichen Mitarbeiter/-innen bzw. der Höhe der Spenden und Subventionen nicht beantwortet wurden. Der Tabelle ist zu entnehmen, dass einige Koeffizienten auf bestimmten Quantilen signifikant sind. Da die Anzahl der Ehrenamtlichen keine signifikanten Ergebnisse aufweist (siehe Tabelle 17), sind auch die Ergebnisse des Ehrenamt Missing Dummys nicht von besonderem Interesse. Der Spenden und Subventions Missing Dummy hat zwei signifikante Ergebnisse, nämlich im 0,1 und 0,25 Lohnquantil. Dies ist ein Hinweis darauf, dass die fehlenden Werte in den Daten dieser zwei Lohnquantile signifikanten Einfluss auf die Ergebnisse haben. Die Aussagen über die 
zwei Variablen „Subventionen“ und „Spenden“ sind daher für diese zwei Lohnquantile möglicherweise verzerrt.

Tabelle 20 zeigt die Ergebnisse der Quantilsschätzung für die Kollektivvertragsvariablen und einige Variable, die die Zusammensetzung der Arbeitnehmer/innen beschreiben.

\section{Tabelle 20: Weitere Ergebnisse der Quantilsregression (Forts.)}

Kollektivvertrag / Zusammensetzung der Arbeitnehmer

\begin{tabular}{|c|c|c|c|c|}
\hline Variable & Koef. Q10 & $\begin{array}{l}\text { Bootstr. } \\
\text { Standard- } \\
\text { fehler }\end{array}$ & Koef. Q25 & $\begin{array}{l}\text { Bootstr. } \\
\text { Standard- } \\
\text { fehler }\end{array}$ \\
\hline $\begin{array}{l}\text { Kein Kollektivvertrag, aber } \\
\text { Orientierung }\end{array}$ & $-0,1353514 * * *$ & $(0,028618)$ & $-0,125325 * * *$ & $(0,0184187)$ \\
\hline kein Kollektivvertrag & $-0,0478227 * * *$ & $(0,0346745)$ & $-0,1033924 * * *$ & $(0,0323955)$ \\
\hline $\begin{array}{l}\text { Kollektivvertrag Missing } \\
\text { Dummy }\end{array}$ & $0,2265478^{* * *}$ & $(0,0197531)$ & $0,192956 * * *$ & $(0,0157198)$ \\
\hline Anteil Teilzeitbeschäftigter & $-0,0355221^{* *}$ & $(0,0175871)$ & $0,0312736 * *$ & $(0,0129796)$ \\
\hline Anteil Geringfügiger & $-0,0522594 * * *$ & $(0,045845)$ & $-0,1277113 * * *$ & $(0,0446447)$ \\
\hline Frauenanteil & $-0,1326335$ & $(0,020003)$ & $-0,0181544$ & $(0,0149305)$ \\
\hline \multirow[t]{2}{*}{ log. VZÄ } & $0,0437373^{* * *}$ & $(0,0061363)$ & $0,0303552^{* * *}$ & $(0,0039119)$ \\
\hline & Koef. Q50 & $\begin{array}{l}\text { Bootstr. } \\
\text { Standard- } \\
\text { fehler }\end{array}$ & Koef. Q75 & $\begin{array}{c}\text { Bootstr. } \\
\text { Standard- } \\
\text { fehler }\end{array}$ \\
\hline $\begin{array}{l}\text { Kein Kollektivvertrag, aber } \\
\text { Orientierung }\end{array}$ & $-0,0887967 * * *$ & $(0,0136284)$ & $-0,1243671 * * *$ & $(0,0165015)$ \\
\hline kein Kollektivvertrag & $-0,1004739 * * *$ & $(0,0222014)$ & $-0,1025358 * * *$ & $(0,0196175)$ \\
\hline $\begin{array}{l}\text { Kollektivvertrag Missing } \\
\text { Dummy }\end{array}$ & $0,1321309 * * *$ & $(0,0105145)$ & $0,0678232 * * *$ & $(0,0123782)$ \\
\hline Anteil Teilzeitbeschäftigter & $0,0581447^{* * *}$ & $(0,0091442)$ & $0,0764883 * * *$ & $(0,0105816)$ \\
\hline Anteil Geringfügiger & $-0,1775413^{* * *}$ & $(0,0217616)$ & $-0,2079252 * * *$ & $(0,0183048)$ \\
\hline Frauenanteil & $0,0462123^{* * *}$ & $(0,0116272)$ & $0,0916107^{* * *}$ & $(0,0103454)$ \\
\hline \multirow[t]{2}{*}{ log. VZA } & $0,0070196 * *$ & $(0,0033642)$ & $-0,0020564$ & $(0,0032403)$ \\
\hline & Koef. Q90 & $\begin{array}{l}\text { Bootstr. } \\
\text { Standard- } \\
\text { fehler }\end{array}$ & & \\
\hline $\begin{array}{l}\text { Kein Kollektivvertrag, aber } \\
\text { Orientierung }\end{array}$ & $-0,1098597^{* * *}$ & $(0,0205356)$ & & \\
\hline kein Kollektivvertrag & $-0,0769206 * * *$ & $(0,0248978)$ & & \\
\hline $\begin{array}{l}\text { Kollektivvertrag Missing } \\
\text { Dummy }\end{array}$ & $0,0533725^{* * *}$ & $(0,0167151)$ & & \\
\hline Anteil Teilzeitbeschäftigter & $0,0266118^{* *}$ & $(0,0125004)$ & & \\
\hline Anteil Geringfügiger & $-0,1574318 * * *$ & $(0,0199518)$ & & \\
\hline Frauenanteil & $0,0636666^{* * *}$ & $(0,0132085)$ & & \\
\hline log. VZÄ & $-0,0143002 * * *$ & $(0,0040524)$ & & \\
\hline
\end{tabular}

*** $\left({ }^{* *}\right)[*]$ am $99 \%-(95 \%-)$ [90\%-] Level signifikant

Quelle: eigene Berechnungen, NPO-Steuerdaten 2006 
Für die Kollektivvertragsvariablen zeigt sich, dass, wenig überraschend, die Löhne in Organisationen, die keinem Kollektivvertrag unterliegen niedriger sind, als in jenen, die einem kollektivvertraglichen Schema unterliegen. Allerdings sind die Koeffizienten bei einigen Quantilen in den Organisationen, die keinem Kollektivvertrag unterliegen, sich jedoch an einem orientieren, niedriger als in den Organisationen, die keinem Kollektivvertrag unterliegen und sich auch an keinem orientieren. Insgesamt sind die Ergebnisse jedoch mit Vorsicht $\mathrm{zu}$ interpretieren, da auch hier die Missing Dummys durchgehend signifikante Ergebnisse aufweisen.

Ein hoher Anteil geringfügiger Beschäftigter senkt die erwartete Lohnhöhe, wobei der Effekt für mittlere Löhne am stärksten ist. Ein hoher Anteil Teilzeitbeschäftigter, die jedoch nicht geringfügig be-schäftigt sind, führt außer im 0,1 Lohnquantil zu höheren Löhnen. Im untersten Lohnquantil sinken die Löhne bei größerem Anteil Teilzeitbeschäftigter. Ein hoher Frauenanteil führt, wie erwartet, zu höheren Löhnen, allerdings gilt das Ergebnis nur für die Lohnquantile $0,5,0,75$ und 0,9 , die übrigen Lohnquantile weisen nicht signifikant von 0 verschiedene Ergebnisse auf. Die logarithmierten Vollzeitäquivalente, was ein Maß für die Größe der Organisation sein soll, führen in den unteren Lohnquantilen zu höheren Löhnen, im obersten Lohnquantil überraschenderweise zu niedrigeren.

Tabelle 21 zeigt die Ergebnisse für die Bundeslands-Dummys, wobei Wien als Referenzbundesland dient. Auch hier sind einige Ergebnisse signifikant. Wie erwartet sind die Löhne, mit einer nicht signifikanten Ausnahme, in den Bundesländern Tirol, Kärnten und Salzburg niedriger als in Wien. Die anderen Bundesländer weisen teils höhere, teil niedrigere Löhne auf.

Tabelle 22 zeigt schließlich die Ergebnisse für einzelne Branchen-Dummys. Referenzkategorie in dieser Tabelle ist die Branche „Sozialwesen a.n.g.“. Einige Branchen weisen über alle Lohnquantile signifikant niedrigere Löhne auf, wie z.B. im Bereich der Kindergärten oder der Hauskrankenpflege. Andere wiederum weisen erwartungs-gemäß durchgehend höhere Löhne auf. Dazu zählen die Branchen „Forschung und Entwicklung im Bereich Rechts-, Wirtschafts- und Sozialwissenschaften sowie Sprach-, Kultur- und Kunst-wissenschaften“, „Altenheime“ und „Erbringung von sonstigen Dienstleistungen des Sports“. Viele Branchen weisen für einzelne Lohnquantile höhere oder niedrigere Löhne im Vergleich zum Sozialwesen auf.

Zusammenfassend kann gesagt werden, dass das Vorhandensein ehrenamtlicher Mitarbeiter/-innen den erwarteten Tageslohn von bezahlten Arbeitskräften um ca. 10\% senkt, die Anzahl der Freiwilligen jedoch unerheblich ist. Spenden erhöhen den Lohn der oberen Lohnsegmente, Subventionen erhöhen die Löhne aller Lohnsegmente. Die beiden letzten Effekte sind jedoch sehr gering. 
Tabelle 21: Weitere Ergebnisse der Quantilsregression (Forts.)

\begin{tabular}{|c|c|c|c|c|}
\hline \multirow[b]{2}{*}{ Variable } & \multicolumn{2}{|c|}{ Bundesländer } & \multirow[b]{2}{*}{ Koef. Q25 } & \multirow[b]{2}{*}{$\begin{array}{c}\text { Bootstr. } \\
\text { Standard- } \\
\text { fehler }\end{array}$} \\
\hline & Koef. Q10 & $\begin{array}{l}\text { Bootstr. } \\
\text { Standard- } \\
\text { fehler }\end{array}$ & & \\
\hline Niederösterreich & $0,1233522 * * *$ & $(0,0244257)$ & $0,0555961^{* * *}$ & $(0,0189759)$ \\
\hline Burgenland & 0,0456895 & $(0,031251)$ & $-0,0252269$ & $(0,0248755)$ \\
\hline Steiermark & $0,1237982^{* * *}$ & $(0,0493441)$ & $0,1321869^{* * *}$ & $(0,032195)$ \\
\hline Kärnten & $-0,007376$ & $(0,0475141)$ & 0,0030275 & $(0,0519924)$ \\
\hline Oberösterreich & $0,2174186^{* * *}$ & $(0,025946)$ & $0,084321^{* * *}$ & $(0,016834)$ \\
\hline Salzburg & $-0,0551007 * * *$ & $(0,0470875)$ & $-0,1978308 * * *$ & $(0,0481402)$ \\
\hline Tirol & $-0,0340743 * * *$ & $(0,0341622)$ & $-0,0859839 * * *$ & $(0,0335196)$ \\
\hline \multirow[t]{2}{*}{ Vorarlberg } & $0,2218309^{* * *}$ & $(0,0424429)$ & $0,1084892^{* * *}$ & $(0,0289283)$ \\
\hline & Koef. Q50 & $\begin{array}{c}\text { Bootstr. } \\
\text { Standard- } \\
\text { fehler }\end{array}$ & Koef. Q75 & $\begin{array}{c}\text { Bootstr. } \\
\text { Standard- } \\
\text { fehler }\end{array}$ \\
\hline Niederösterreich & $-0,0890676^{* * *}$ & $(0,0141577)$ & $-0,1175814^{* * *}$ & $(0,016161)$ \\
\hline Burgenland & $-0,1627419^{* * *}$ & $(0,0209776)$ & $-0,2037352^{* * *}$ & $(0,0183336)$ \\
\hline Steiermark & $-0,0255534$ & $(0,0227208)$ & $-0,0724353^{* * *}$ & $(0,0257545)$ \\
\hline Kärnten & $-0,113525^{* * *}$ & $(0,0281868)$ & $-0,1776721^{* * *}$ & $(0,0287011)$ \\
\hline Oberösterreich & $-0,0082775$ & $(0,0121349)$ & $-0,0231784^{*}$ & $(0,0123415)$ \\
\hline Salzburg & $-0,2167543 * * *$ & $(0,0324756)$ & $-0,039866$ & $(0,0297257)$ \\
\hline Tirol & $-0,1148751 * * *$ & $(0,0207869)$ & $-0,1177497^{* * *}$ & $(0,0164163)$ \\
\hline \multirow[t]{2}{*}{ Vorarlberg } & $-0,0247537$ & $(0,022079)$ & $-0,0684922^{* * *}$ & $(0,0213093)$ \\
\hline & Koef. Q90 & $\begin{array}{l}\text { Bootstr. } \\
\text { Standard- } \\
\text { fehler }\end{array}$ & & \\
\hline Niederösterreich & $-0,1170853^{* * *}$ & $(0,0262823)$ & & \\
\hline Burgenland & $-0,22115333^{* * *}$ & $(0,0275624)$ & & \\
\hline Steiermark & $-0,0396408$ & $(0,028818)$ & & \\
\hline Kärnten & $-0,2072511 * * *$ & $(0,032603)$ & & \\
\hline Oberösterreich & $-0,0219261$ & $(0,0158848)$ & & \\
\hline Salzburg & $-0,0157226$ & $(0,0304673)$ & & \\
\hline Tirol & $-0,0964532 * * *$ & $(0,0237695)$ & & \\
\hline Vorarlberg & $-0,070173^{* * *}$ & $(0,0234534)$ & & \\
\hline
\end{tabular}




\section{Tabelle 22: Weitere Ergebnisse der Quantilsregression (Forts.)}

\section{ONACE Klassifikation}

\begin{tabular}{|c|c|c|c|c|}
\hline Variable & Koef. Q10 & $\begin{array}{l}\text { ootstr. Standard } \\
\text { fehler }\end{array}$ & Koef. Q25 & $\begin{array}{l}\text { Bootstr. Standard- } \\
\text { fehler }\end{array}$ \\
\hline Restaurants und Gasthäuser & 0,0598752 & $(0,0175747)$ & $-0,0342821$ & $(0,0485982)$ \\
\hline Buffets und Imbissstuben & $-0,3680127 * * *$ & $(0,1233865)$ & $-0.5761164 * * *$ & $(0,194474)$ \\
\hline $\begin{array}{l}\text { F\&E im Bereich Natur-. Ingenieur-, } \\
\text { Agrarwissenschaften und Medizin }\end{array}$ & -0.0292463 & $(0,1457458)$ & $-0,1947429$ & $(0,3120945)$ \\
\hline $\begin{array}{l}\text { F\&E im Bereich Rechts-, Wirtschaffs- und } \\
\text { Sozialwissenschaften sowie Sprach-, Kultur- } \\
\text { und Kunstwissenschaften }\end{array}$ & $0,3673849 * * *$ & $(0,2249689)$ & $0,2452186^{* * *}$ & $(0,0812916)$ \\
\hline Rechtsberatung & $-0,5043546$ & $(0,4083656)$ & $-0,7003895$ & $(0,5075182)$ \\
\hline Architekturbliros & $0,9682833^{* * *}$ & $(0,1756219)$ & $0,6965897^{* * *}$ & $(0,1369819)$ \\
\hline $\begin{array}{l}\text { Technische, physikalische und chemische } \\
\text { Untersuchung }\end{array}$ & 0,1382456 & $(0,2178779)$ & 0,1411198 & $(0,2493863)$ \\
\hline Allgemeine öffentliche Verwaltung & $0.5490817^{* * *}$ & $(0.0317764)$ & $0,208441^{* * *}$ & $(0,0784453)$ \\
\hline Kindergärten & $-0.1680372 * * *$ & $(0,0293377)$ & $-0,1175524 * * *$ & $(0,0228164)$ \\
\hline Volksschulen & $-0,5760617$ & $(0,570697)$ & 0.1597315 & $(0,6500859)$ \\
\hline Berufsbildende mittlere Schulen & $-0,7118492 * * *$ & $(0,064686)$ & $-0,7410888 * * *$ & $(0,1213967)$ \\
\hline $\begin{array}{l}\text { Hochschulen und hochschulverwandte } \\
\text { Lehranstalten }\end{array}$ & 0,1055714 & $(0.2068243)$ & $-0,0431388$ & $(0.2404936)$ \\
\hline Erwachsenenbildung und Unterricht a.n.g. & 0,1850439 & $(0.042379)$ & 0,039423 & $(0,0326409)$ \\
\hline Krankenhäuser & $0.3796795^{* * *}$ & $(0.0281538)$ & $0.189865^{* * *}$ & $(0,0287265)$ \\
\hline Zahnambulatorien & $0,4172557^{* * *}$ & $(0,1452956)$ & $0.4989551^{* * *}$ & $(0,181214)$ \\
\hline Krankentransporte und Rettungsdienste & $0,3582262 * * *$ & $(0,0605905)$ & $0,3313358^{* * *}$ & $(0,0401527)$ \\
\hline Hauskrankenpflege & $-0.0993849 * * *$ & $(0,0361006)$ & $-0,1002073 * * *$ & $(0,0187302)$ \\
\hline $\begin{array}{l}\text { Psychotherapeuten, Klinische und } \\
\text { Gesundheitspsychologen }\end{array}$ & $-0,0631108$ & $(0.274622)$ & 0,0897561 & $(0,3259346)$ \\
\hline Sonstiges Gesundheitswesen a.n.g. & $0.4273304^{* *}$ & $(0,2916378)$ & $0,367639 * *$ & $(0,1621304)$ \\
\hline Altenheime & $0.2382001^{* * *}$ & $(0.0183125)$ & $0,1482295^{* * *}$ & $(0,0167431)$ \\
\hline $\begin{array}{l}\text { Sonstige Heime (ohne Fremden-, Erholungs- } \\
\text { und Ferienheime) }\end{array}$ & $0.0706911^{* * *}$ & $(0,0394701)$ & $0,0961421^{* * *}$ & $(0,0199572)$ \\
\hline Berufsorganisationen & 0.0601519 & $(0,2137002)$ & 0,0086186 & $(0,2045902)$ \\
\hline $\begin{array}{l}\text { Kirchliche und sonstige religiöse } \\
\text { Vereinigungen }\end{array}$ & -0.0294405 & $(0,0450731)$ & $-0,0257569$ & $(0,0328029)$ \\
\hline $\begin{array}{l}\text { Sonstige Interessenvertretungen und Vereine } \\
\text { a.n.g. }\end{array}$ & $-0.0551339^{* *}$ & $(0,0326622)$ & $-0,0522737^{* *}$ & $(0,0212954)$ \\
\hline $\begin{array}{l}\text { Künstlerische und schriftstellerische } \\
\text { Tătigkeiten und Darbietungen }\end{array}$ & $-0.4349965 * * *$ & $(0.1321819)$ & $-0,9583845 * * *$ & $(0,09755)$ \\
\hline $\begin{array}{l}\text { Betrieb und technische Hilfsdienste für } \\
\text { kulturelle und unterhaltende Leistungen }\end{array}$ & $-0.0617868 *$ & $(0,1491233)$ & $-0,2454167^{*}$ & $(0.1370323)$ \\
\hline Bibliotheken und Archive & -0.2608886 & $(0.3703826)$ & 0,1483785 & $(0,1576169)$ \\
\hline Museen und Denkmalschutz & $0.0529544^{*}$ & $(0,1516932)$ & $-0,1855689^{*}$ & $(0,1000475)$ \\
\hline $\begin{array}{l}\text { Botanische und zoologische Gärten sowie } \\
\text { Naturparks }\end{array}$ & $0.6972827^{* * *}$ & $(0.3822291)$ & $0,7730453^{* * *}$ & $(0,1594409)$ \\
\hline Betrieb von sonstigen Sportanlagen & 0.3858831 & $(0.0895855)$ & 0,1968457 & $(0,1547042)$ \\
\hline $\begin{array}{l}\text { Erbringung von sonstigen Dienstleistungen des } \\
\text { Sports }\end{array}$ & $0,039419^{* * *}$ & $(0,0720741)$ & $0,1981338^{* * *}$ & $(0.0545958)$ \\
\hline Konstante & $0.6576118^{* * *}$ & $(0,0880248)$ & $1,400104^{* * * *}$ & $(0,0710633)$ \\
\hline
\end{tabular}


Bootstr. Standard-

Sonstiges Beherbergungswesen a.n.g.
Restaurants und Gasthăuser
Buffets und Imbissstuben
F\&E im Bereich Natur-. Ingenieur-,
Agrarwissenschaften und Medizin

F\&E im Bereich Rechts-, Wirtschafts- und Sozialwissenschaften sowie Sprach-, Kulturund Kunstwissenschaften

Rechtsberatung

Architekturbüros

Technische, physikalische und chemische Untersuchung

Allgemeine offentliche Verwaltung

Kindergărten

Volksschulen

Berufsbildende mittlere Schulen

Hochschulen und hochschulverwandte

Lehranstalten

Envachsenenbildung und Unterricht a.n.g.

Krankenhăuser

Zahnambulatorien

Krankentransporte und Rettungsdienste

Hauskrankenpflege

Psychotherapeuten, Klinische und

Gesundheitspsychologen

Sonstiges Gesundheitswesen a.n.g.

Altenheime

Sonstige Heime (ohne Fremden-, Erholungsund Ferienheime)

Berufsorganisationen

Kirchliche und sonstige religiöse Vereinigungen

Sonstige Interessenvertretungen und Vereine a.n.g.

Künstlerische und schriftstellerische

Tatigkeiten und Darbietungen

Betrieb und technische Hilfsdienste für kulturelle und unterhaltende Leistungen

Bibliotheken und Archive

Museen und Denkmalschutz

Botanische und zoologische Gärten sowie Naturparks

Betrieb von sonstigen Sportanlagen

Erbringung von sonstigen Dienstleistungen des Sports

Konstante

\begin{tabular}{|c|c|c|c|}
\hline Koef. Q50 & $\begin{array}{c}\text { Bootstr. Standard- } \\
\text { fehler }\end{array}$ & Koef. Q75 & $\begin{array}{c}\text { Bootstr. Standard- } \\
\text { fehler }\end{array}$ \\
\hline$-0,0227446$ & $(0,0295318)$ & $-0,0447477$ & $(0,0260756)$ \\
\hline$-0.1323998 * * *$ & $(0,035371)$ & $-0,2422704 * * *$ & $(0,0313026)$ \\
\hline$-0.6747009^{* * *}$ & $(0,222024)$ & $-0,3500967$ & $(0,4610984)$ \\
\hline 0,1840946 & $(0,12617)$ & $0,1037766^{*}$ & $(0,0578153)$ \\
\hline $0,175684^{* * *}$ & $(0,0685332)$ & 0,1417632 *** & $(0,0472402)$ \\
\hline 0.2948026 & $(0,7612071)$ & 0,1527561 & $(0,7155997)$ \\
\hline $0,4313948^{* * *}$ & $(0,0908512)$ & $0,2350903^{* * *}$ & $(0,0673721)$ \\
\hline 0,1150203 & $(0,1934897)$ & 0,0882013 & $(0,2651614)$ \\
\hline 0.0711306 & $(0,1069993)$ & 0,0128088 & $(0,1446564)$ \\
\hline$-0,0691582 * * *$ & $(0,0106172)$ & $-0,0900203 * * *$ & $(0,0067675)$ \\
\hline 0,0280276 & $(0,1813369)$ & $-0,1595919^{* *}$ & $(0,0759584)$ \\
\hline$-0,2258014^{* * *}$ & $(0,0520482)$ & $-0,0284394$ & $(0,0379667)$ \\
\hline 0,0843272 & $(0,2061506)$ & 0,3645732 & $(0,2325864)$ \\
\hline $0,0538409 * *$ & $(0,0231637)$ & $0,1033805^{* * *}$ & $(0,0269559)$ \\
\hline 0.0317194 & $(0.0210712)$ & $-0,0224461$ & $(0,0220672)$ \\
\hline $0,2850962^{* * *}$ & $(0.1001125)$ & $0,1693635^{* * *}$ & $(0,0582541)$ \\
\hline $0,2960692 * * *$ & $(0,0333996)$ & $0,2424267 * * *$ & $(0,0275427)$ \\
\hline$-0,0541969 * * *$ & $(0.0118016)$ & $-0,0870714^{* * *}$ & $(0,0143258)$ \\
\hline $0,3350661 * *$ & $(0,1658858)$ & $0,183077 * * *$ & $(0,0669742)$ \\
\hline$-0,0300722$ & $(0,0546533)$ & $-0,1075836$ & $(0,102757)$ \\
\hline $0.1131722^{* * * *}$ & $(0,0119735)$ & $0,0875479 * * *$ & $(0,0131834)$ \\
\hline $0,0887874^{* * *}$ & $(0,0166674)$ & $0,0803906^{* * *}$ & $(0,0123177)$ \\
\hline $0,2232718^{* *}$ & $(0,1035992)$ & $0,1621569 * * *$ & $(0,0633483)$ \\
\hline $0.0662644^{* * *}$ & $(0,0196102)$ & 0,028586 & $(0,0184629)$ \\
\hline $0,0286563^{*}$ & $(0,0173077)$ & $0,0714297 * \cdots$ & $(0,0170783)$ \\
\hline$-1,441326^{*}$ & $(0,1923081)$ & $-0,3894255$ & $(0,5027552)$ \\
\hline$-0,4485841 * * *$ & $(0,1690633)$ & 0,0142347 & $(0,1122221)$ \\
\hline 0,0913159 & $(0.0667657)$ & 0,0580376 & $(0,080493)$ \\
\hline$-0,0258673$ & $(0.0955308)$ & $0,1514113^{* * *}$ & $(0,0585117)$ \\
\hline $0.6130616^{* * *}$ & $(0,0683545)$ & $0,3778719 * \cdots$ & $(0,065145)$ \\
\hline 0,2423101 & $(0,2332826)$ & $-0,007248$ & $(0,2398116)$ \\
\hline $0,2895202^{* * *}$ & $(0.0551066)$ & $0,4969226^{* * *}$ & $(0,0814133)$ \\
\hline $2,519647^{* * *}$ & $(0,0408035)$ & $2,850809^{* * *}$ & $(0,0349628)$ \\
\hline
\end{tabular}




\begin{tabular}{|c|c|c|}
\hline & Koef. Q90 & $\begin{array}{l}\text { Bootstr. Standard- } \\
\text { fehler }\end{array}$ \\
\hline Sonstiges Beherbergungswesen a.n.g. & $-0,1349799 * * *$ & $(0,0438502)$ \\
\hline Restaurants und Gasthauser & $-0,3103948 * * *$ & $(0,0790808)$ \\
\hline Buffets und Imbissstuben & 0,1139685 & $(0.6069099)$ \\
\hline $\begin{array}{l}\text { F\&E im Bereich Natur-, Ingenieur-, } \\
\text { Agrarwissenschaften und Medizin }\end{array}$ & 0,0933926 & $(0,0698811)$ \\
\hline $\begin{array}{l}\text { F\&E im Bereich Rechts-, Wirtschafts- und } \\
\text { Sozialwissenschaften sowie Sprach-, Kultur- } \\
\text { und Kunstwissenschaften }\end{array}$ & $0,1663783^{*}$ & $(0,0903073)$ \\
\hline Rechtsberatung & 0,5642287 & $(0,5123111)$ \\
\hline Architekturbüros & $-0,0650352$ & $(0,0708536)$ \\
\hline $\begin{array}{l}\text { Technische, physikalische und chemische } \\
\text { Untersuchung }\end{array}$ & 0,2599729 & $(0,2227593)$ \\
\hline 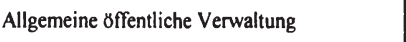 & 0,0425573 & $(0,0642264)$ \\
\hline Kindergărten & $-0.0900196 * * *$ & $(0,0090222)$ \\
\hline Volksschulen & $-0,3180986 * * *$ & $(0,0779555)$ \\
\hline Berufsbildende mittlere Schulen & 0,0027655 & $(0,0313001)$ \\
\hline $\begin{array}{l}\text { Hochschulen und hochschulverwandte } \\
\text { Lehranstalten }\end{array}$ & 0,2103067 & $(0,1702777)$ \\
\hline Erwachsenenbildung und Unterricht a.n.g. & 0,0454922 & $(0,0337467)$ \\
\hline Krankenhăuser & $-0,0036173$ & $(0,0425833)$ \\
\hline Zahnambulatorien & 0,0997963 & $(0,0761796)$ \\
\hline Krankentransporte und Rettungsdienste & 0,0368524 & $(0,0351457)$ \\
\hline Hauskrankenpflege & $-0,116195^{* * *}$ & $(0,015576)$ \\
\hline $\begin{array}{l}\text { Psychotherapeuten, Klinische und } \\
\text { Gesundheitspsychologen }\end{array}$ & 0,1979255 & $(0,1287766)$ \\
\hline Sonstiges Gesundheitswesen a.n.g. & $-0,1302533$ & $(0,1644855)$ \\
\hline Altenheime & $0,046987^{* * *}$ & $(0,0162748)$ \\
\hline $\begin{array}{l}\text { Sonstige Heime (ohne Fremden-, Erholungs- } \\
\text { und Ferienheime) }\end{array}$ & $0,0312403 * *$ & $(0,0146313)$ \\
\hline Berufsorganisationen & 0,0745126 & $(0,0858506)$ \\
\hline $\begin{array}{l}\text { Kirchliche und sonstige religiøse } \\
\text { Vereinigungen }\end{array}$ & $-0,0472645^{*}$ & $(0,0270874)$ \\
\hline $\begin{array}{l}\text { Sonstige Interessenvertretungen und Vereine } \\
\text { a.n.g. }\end{array}$ & 0,0044601 & $(0,0236955)$ \\
\hline $\begin{array}{l}\text { Künstlerische und schriftstellerische } \\
\text { Tătigkeiten und Darbietungen }\end{array}$ & $-0,097337$ & $(0,401774)$ \\
\hline $\begin{array}{l}\text { Betrieb und technische Hilfsdienste für } \\
\text { kulturelle und unterhaltende Leistungen }\end{array}$ & $-0,103202$ & $(0,1021457)$ \\
\hline Bibliotheken und Archive & 0,0548821 & $(0,1239459)$ \\
\hline Museen und Denkmalschutz & 0,0143811 & $(0,0541974)$ \\
\hline $\begin{array}{l}\text { Botanische und zoologische Gärten sowie } \\
\text { Naturparks }\end{array}$ & 0,3077502 & $(0,1987393)$ \\
\hline Betrieb von sonstigen Sportanlagen & $-0,0396018$ & $(0,1398428)$ \\
\hline $\begin{array}{l}\text { Erbringung von sonstigen Dienstleistungen des } \\
\text { Sports }\end{array}$ & $0,3601792^{* * *}$ & $(0,0436732)$ \\
\hline Konstante & 3,18424 & $-(0,0558668)$ \\
\hline
\end{tabular}




\subsection{Beantwortung der Forschungsfragen}

Im ersten Teil der Dissertation soll die Frage beantwortet werden, welche Determinanten die Lohnhöhe von bezahlten Arbeitnehmer/-innen im Nonprofit Sektor beeinflussen. Der Fokus liegt dabei auf der Frage, ob ehrenamtliche Arbeit, Spenden und Subventionen die Lohnhöhe beeinflussen.

Ein direkter Vergleich der Ergebnisse der beiden Untersuchungsmethoden erscheint nicht sinnvoll, da die Lohnfunktion bei diesen beiden Methoden auf sehr unterschiedliche Art geschätzt wird.

Insgesamt zeigt sich, dass (freiwillige) Zuwendungen an NPO in Form von ehrenamtlicher Arbeit, Spenden und Subventionen zum Teil Einfluss auf die Lohnhöhe bezahlter Arbeitskräfte im österreichischen Nonprofit Sektor haben. Anhand der OLS-Regression zeigt sich, dass nach ausführlicher Kontrolle von individuellen und Organisations-unterschieden NPO, in denen auch freiwillige Mitarbeiter/-innen tätig sind, um ca. $10 \%$ weniger zahlen als solche Organisationen, in denen keine Ehrenamtlichen arbeiten. Die Anzahl der freiwilligen Mitarbeiter/-innen hingegen hat keinen Erklärungswert. Außerdem steigt der Lohn mit zunehmender Höhe der Subventionen, die die Organisation erhält. Jeder zusätzliche Euro an Subventionen, der an die Organisationen geht, erhöht den Lohn der bezahlten Arbeitskräfte um 0,0001\%. Eine für unterschiedliche Lohnhöhe differenzierte Betrachtung, die anhand der Quantilsregression möglich ist, zeigt, dass der Effekt des Ehrenamtsdummys an den Rändern der Lohnverteilung niedriger ist als in der Mitte. Auch hier haben die Anzahl der freiwilligen Mitarbeiter/-innen keinen signifikanten Einfluss. Im Unterschied zur OLS-Regression weist die Spendenvariable bei dieser Schätzung für einige Quantile signifikante Ergebnisse auf. Am oberen Ende der Lohnverteilung führen höhere Spenden zu höheren Löhnen. Subventionen führen auf allen geschätzten Lohnquantilen zu höheren Löhnen. Die Koeffizienten beider Variablen sind jedoch sehr klein, so zeigt sich, dass ein zusätzlicher Subventions-Euro zu einer Lohnsteigerung um 0,0000708\% am 0,1 Lohnquantil und um 0,000114\% am 0,9 Lohnquantil führt. Sowohl im Fall der Subventionen als auch im Fall der Spenden profitieren die höheren Lohnklassen mehr von höheren Zahlungen.

Wie lassen sich die Ergebnisse erklären? Leider ist es nicht möglich, die Ergebnisse auf jeweils eine Hypothese zurückzuführen, da für eine genaue Unterscheidung zwischen Erklärungsansätzen zusätzliche Kontrollvariablen fehlen. Der negative Einfluss von Ehrenamtlichen auf die Lohnhöhe kann einerseits mit der Arbeitsspendenhypothese erklärt werden. Individuen, die sich freiwillig in einer NPO betätigen, können ein Indikator für die soziale Erwünschtheit des Gutes der NPO sein. Weitere Ansätze zur Erklärung sind eine mit freiwilligen Mitarbeiter/-innen verbundene geringere Anforderung an die Qualifikation der bezahlten Mitarbeiter/-innen oder eine geringere Notwendigkeit, Effizienzlöhne zu zahlen. Das Vorhandensein von freiwilligen Mitarbeiter/-innen kann außer- 
dem die Verhandlungsmacht der bezahlten Arbeitskräfte schwächen. Die positiven Koeffizienten der Spenden- und Subventionsvariable können mit der RentSharing Theorie erklärt werden. Höhere Subventionen und Spenden eröffnen mehr Möglichkeiten, die zusätzlichen Mittel mit der Belegschaft zu „teilen“, d.h. höhere Löhne zu bezahlen. 
Teil II:

Determinanten der innerbetrieblichen Lohnstreuung im Nonprofit Sektor 
Astrid Haider - 978-3-631-75377-4

Downloaded from PubFactory at 01/11/2019 05:43:53AM

via free access 
Um ein größeres Verständnis für die Entlohnung im Nonprofit Sektor zu bekommen, sind nicht nur die Lohnhöhe und deren Determinanten von Interesse. Der zweite Teil dieser Arbeit gibt weiterführenden Einblick in die Lohnstruktur von NPO, indem nach der Bestimmung der Determinanten der individuellen Lohnhöhe eines Arbeitnehmers bzw. einer Arbeitnehmerin in einer NPO nun die Lohnstreuung innerhalb der Organisation analysiert wird. Wenn Ehrenamt, Spenden und Sub-ventionen die Lohnhöhe einer NPO beeinflussen, so liegt es nahe, dass auch Unterschiede für die Lohnstreuung einer NPO bestehen. Für diese Arbeit stellt sich also die Frage, ob zeitliche und monetäre Transfers, die, wie im Teil I gezeigt, die Lohnhöhe beeinflussen, auch Auswirkungen auf die Lohnstreuung in einer Organisation haben.

Statt des Individuums als zentraler Untersuchungseinheit wird nun die Organisation in den Mittelpunkt der Analyse gestellt.

Im einleitenden Kapitel des zweiten Teils der Dissertation wird zuerst eine Begriffsklärung vorgenommen, danach werden die genauen Fragestellungen dieses Teils der Dissertation vorgestellt, und es wird eine kurze Übersicht über die Vorgehensweise gegeben.

\subsection{Lohnstreuung zwischen und in Organisationen}

Lohnstreuung bezeichnet eine Abweichung des Lohns vom Durchschnittslohn aufgrund unterschiedlicher Ausprägungen indi-vidueller Merkmale der Arbeitnehmer/-innen oder bestimmter Eigenschaften der Arbeitgeber/-innen (vgl. Hiller 2006: 285). Unter Lohnstreuung wird ebenso wie mit dem Begriff ,Lohnspanne' die Differenz zwischen hohen und niedrigen Löhnen verstanden.

Einerseits gibt es Lohnstreuung zwischen Organisationen (zwischenbetriebliche Lohnstreuung). Sie wird auch als Firmenlohndifferenzial bezeichnet. Dabei wird geprüft, inwieweit Arbeitnehmer/-innen einer Organisation einen höheren Lohn erhalten als sie für die gleiche Tätigkeit in einer anderen Organisation erhalten hätten bzw. inwieweit sich die mittleren Löhne der Firmen voneinander unterscheiden (vgl. Stephan 2001: 13).

Zum anderen gibt es innerhalb einer Organisation Unterschiede in der $\mathrm{Be}$ zahlung für verschiedene Tätigkeiten (innerbetriebliche Lohnstreuung bzw. innerbetriebliches Lohndifferenzial oder Lohnspreizung). Auch die Lohnstreuung innerhalb einer Organisation trägt wesentlich zur Gesamt-streuung der Löhne

Die Streuung der Löhne gibt einerseits Rückschluss auf die Ungleichheit in einer Gesellschaft, eine hohe Varianz der Löhne deutet auf hohe Lohnungleichheit. Zweitens kann von der Streuung auf die Produktions-funktion der Organi- 
sation geschlossen werden (vgl. Lazear, Shaw 2007: 25f.). Wenn z.B. die Lohnstreuung innerhalb von Organisationen sehr gering ist bzw. keine innerbetriebliche Lohnstreuung vorhanden ist, ist eine Interpretation, dass auch die Arbeitnehmer/-innen sehr homogen bzw. fast identisch in Bezug auf ausschlaggebende Charakteristika sind. Dies kann so interpretiert werden, dass die Produktion von Gütern innerhalb einer Firma ähnliche Qualifikationen erfordert bzw. Arbeitsteilung zwischen und nicht innerhalb Organisationen stattfindet. Drittens kann aufgrund der innerbetrieblichen Lohnstreuung auf technologischen Wandel rückgeschlossen werden. Eine Beobachtung von Änderungen der Lohnstreuung über die Zeit kann zeigen, ob es zunehmend zu einer technologisch bedingten Trennung von hoch- und niedrigqualifizierten Arbeitnehmer/-innen kommt (vgl. Kremer, Maskin 1996). Viertens ist Lohnstreuung in Hinsicht auf die Auswirkungen auf die Produktivität der Arbeitnehmer/-innen von Interesse (vgl. Lazear, Shaw 2007: 26).

Während Lohnunterschiede zwischen Branchen oder zwischen Unternehmen schon länger Untersuchungsgegenstand sind, ist die innerbetriebliche Lohnstreuung noch ein relativ unerforschtes Gebiet. Als Grund kann die mangelnde Datenverfügbarkeit genannt werden (vgl. Lazear, Shaw 2007). Erst die Verfügbarkeit von (möglichst vollständigen) Daten zu Arbeitgeber/-inne/n und allen Arbeitnehmer/-inne/n innerhalb der Organisationen lässt genaue Untersuchungen zu. Analysen zur innerbetrieblichen und zwischenbetrieblichen Lohnstreuung wurden in einzelnen Ländern bereits mit LEE-Daten durchgeführt (vgl. beispielsweise dazu die verschiedenen Beiträge im Sammelband von Lazear, Shaw 2007), so auch in Österreich (Winter-Ebmer, Zweimüller 1999), wo der Zusammenhang zwischen Lohnstreuung und Produktivität untersucht wurde. Wenn in dieser Arbeit nicht explizit etwas anderes genannt wird, wird Lohnstreuung immer als innerbetriebliche Lohnstreuung verstanden und nicht als zwischen-betriebliche. Das Hauptinteresse des zweiten Teils der Dissertation ist die innerbetriebliche Lohnstreuung bzw. Lohnspreizung innerhalb einer Organisation. ${ }^{50}$ In der vorliegenden Arbeit wird nun die innerbetriebliche Lohnstreuung in einem bestimmten institutionellen Sektor, nämlich dem Nonprofit Sektor, näher untersucht. Auch in diesem Teil liegt das Hauptinteresse auf dem Einfluss nonprofit-spezifischer Faktoren, wie Zuwendungen in Form von ehrenamtlicher Arbeit, Spenden und Subventionen, die die zeitlichen und monetären Ressourcen einer Organisation erweitern, auf die innerbetriebliche Lohnstreuung in NPO.

Die Streuung der Löhne innerhalb einer Volkswirtschaft entsteht aufgrund unterschiedlicher Ausprägungen individueller Merkmale der Arbeitnehmer/-innen, jedoch auch aufgrund verschiedener Eigenschaften der Arbeitgeber/-innen (vgl.

50 In der Arbeit werden die Begriffe innerbetriebliche Lohnstreuung und Lohnspreizung synonym verwendet. 
Hiller 2006: 285; Pfeffer, Davis-Blake 1990: 38). Das heißt, die Streuung der Löhne in einer Volkswirtschaft beruht einerseits auf Lohnunterschieden zwischen einzelnen Organisationen und Unterschieden in der Entlohnung innerhalb einer Firma. Lohnunterschiede entstehen einerseits aufgrund kollektiver Lohnverhandlungen beispielsweise auf Branchenebene oder regionaler Ebene. Doch auch die einzelnen Firmen tragen zur Lohnstreuung in einer Gesellschaft bei. So werden Löhne oft individuell zwischen Arbeitgeber/-in und Arbeitnehmer/-in verhandelt, und Beförderungen werden durch Organisationen vorgenommen. Ungleichheiten auf regionaler Ebene oder Branchenlevel spiegeln in Folge auch die durch Organisationen gemachten Unterschiede wieder (vgl. Pfeffer, DavisBlake 1990: 38).

Zerlegt man die Gesamtstreuung der Löhne in einer Volkswirtschaft in die zwischen- und innerbetriebliche Lohnstreuung, so sind zwei Extremfälle denkbar. Auf der einen Seite ist ein Fall möglich, wo keine zwischenbetriebliche Lohnstreuung vorherrscht und die gesamte Lohnstreuung einer Volkswirtschaft durch die Lohnstreuung innerhalb der Firmen erklärt wird. In dem Fall wäre es für ein Individuum in Bezug auf den Lohn egal, wo es arbeitet, da alle Firmen identisch sind. Ausschlaggebend für die Höhe des Lohns ist die Position innerhalb der Firma, die das Individuum einnimmt. Die Varianz der Löhne innerhalb jeder Firma bestimmt die Varianz der Löhne in der Volkswirtschaft. Im anderen Extremfall unterscheiden sich alle Firmen in ihrer Bezahlung voneinander, innerhalb der Firma ist die Lohnstreuung jedoch sehr klein bzw. gar nicht vorhanden, d.h. alle Individuen innerhalb der Firma werden gleich bezahlt. So gibt es Hochlohn- und Niedriglohnfirmen, die Lohnstreuung in einer Volkswirtschaft entsteht durch die Streuung der Mittelwerte der Löhne der Firmen (vgl. Lazear, Shaw 2007: 7).

Die Lohnstreuung kann demnach folgendermaßen in eine innerbetriebliche und zwischenbetriebliche Lohnstreuung zerlegt werden (vgl. Lazear, Shaw 2007: 7):

$$
\sigma^{2}=\sum_{j=1}^{J} p_{j} \sigma_{i}^{2}+\sum_{j=1}^{J} p_{j}\left(\bar{w}_{j}-\overline{\bar{w}}\right)^{2}
$$

wobei $p$, der Anteil der Arbeitnehmer/-innen in einer Volkswirtschaft ist, der in der Firma $j$ arbeitet, $\sigma_{j}{ }^{2}$ die Varianz der Löhne in der Firma $j, \bar{w}$, der mittlere Lohn der Firma $j$ und $w$ der Durchschnittslohn in der gesamten Volkswirtschaft ist. Die Gesamtvarianz ist also hoch, wenn entweder die durchschnittlichen Löhne zwischen den Firmen stark streuen, wenn also $\left|\bar{w}_{,}-\bar{w}\right|$ groß ist, oder wenn die innerbetriebliche Varianz $\sigma$, hoch ist, oder beides.

Die Realität liegt natürlich zwischen den beiden oben beschriebenen Extremfällen, d.h. ein Teil der gesamten Lohnstreuung in einer Volkswirtschaft, kann durch innerbetriebliche, ein weiterer Teil durch zwischenbetriebliche Lohnstreuung erklärt werden (vgl. Lazear, Shaw 2007: 24). 
Zur Größenordnung der Anteile der zwischenbetrieblichen- und innerbetrieblichen Lohnstreuung an der Lohnstreuung über alle Individuen liegen unterschiedliche empirische Ergebnisse vor, was vermutlich auf Unterschiede in den verwendeten Daten sowie auf die Verschiedenartigkeit der Länder und der Zeitpunkte, an denen die Lohnstreuung untersucht wurde, zurückzuführen ist. Bezüglich der innerbetrieblichen Lohnstreuung stellen Lazear und Shaw (2007) im einleitenden Kapitel ihres Herausgeberbandes, der mehrere Analysen zu Lohnstreuung in verschiedenen Ländern umfasst, fest, dass die innerbetriebliche Lohnstreuung $60 \%$ bis $80 \%$ der gesamten Lohnstreuung über alle Individuen in einer Volkswirtschaft erklärt. Sie bemerken auch, dass die zwischenbetriebliche Lohnstreuung weniger bedeutend ist und stellen die innerbetriebliche Lohnstreuung in den Vordergrund (vgl. Lazear, Shaw 2007: 9). Bezüglich des Stellenwerts der Lohnstreuung zwischen Firmen schätzt beispielsweise Simon (2005: 286) in einem internationalen Vergleich, dass Firmendummys zwischen $35 \%$ und $62 \%$ der gesamten Lohnstreuung erklären, Bronars und Famulari (1997: 296) schätzen denselben Betrag auf bis zu 45\%, Groshen (1991b: 874) zufolge erklären Firmen - je nach Branche - zwischen 12\% und $48 \%$ der Gesamtstreuung der Löhne.

Im Zeitverlauf wird für viele Volkswirtschaften weltweit eine Zunahme der Lohnstreuung in den vergangenen Jahrzehnten konstatiert (vgl. z.B. Cardoso 1997; Lemieux 2002: 647; Salvanes et al. 1999), so auch in Österreich (vgl. Gusenleitner et al. 1998). Während sie in den 1950er und 1960er Jahren relativ konstant war, stieg die Ungleichheit ab den 1970er Jahren laufend. Die wachsende Ungleichheit dürfte vorwiegend durch eine zunehmende zwischenbetriebliche Ungleichheit zu erklären sein (vgl. Lazear, Shaw 2007: 6). Während die Lohnspreizung generell in vielen Ländern in den letzten beiden Jahrzehnten zugenommen hat, blieb die innerbetriebliche Lohnspreizung eher konstant (vgl. z.B. für Schweden Nordström Skans et al. 2007). Daraus kann geschlussfolgert werden, dass eine steigende zwischenbetriebliche Lohnstreuung für die wachsende generelle Lohnstreuung verantwortlich ist. Sie ist außerdem im nicht-produzierenden Gewerbe größer als im produzierenden (vgl. Davis, Haltiwanger 1991: 136). Allerdings stellt Cardoso (1997: 542) widersprüchlich dazu fest, dass die wachsende Lohnungleichheit zu einem großen Teil durch wachsende innerbetriebliche Lohnstreuung verursacht wird. Gründe für die Zunahme der Lohnstreuung werden in Veränderungen des Angebots und der Nachfrage nach den Qualifikationen der Arbeitnehmer/-innen gesehen, sowie in Änderungen der Technologie (vgl. Lemieux 2002: 647). Höhere Qualifikation wird zunehmend höher vergolten (vgl. Cardoso 1997: 542). 


\subsection{Fragestellungen und Vorgehensweise}

Wie auch im ersten Teil der Arbeit liegt das Interesse bei den Determinanten der innerbetrieblichen Lohnstreuung. Dabei wird besonderes Augenmerk auf freiwillige Zuwendungen an NPO gewendet. Untersucht wird, ob „Freiwilligkeit“, die ein Definitionskriterium einer NPO ist, die Lohnstreuung beeinflusst. „Freiwilligkeit“ wird als freiwillige Spende in Form von Zeit oder Geld verstanden. Die Fragestellungen des zweiten Teils der Dissertation lauten daher:

- Welche Determinanten beeinflussen grundsätzlich die innerbetriebliche Lohnstreuung von NPO?

- Beeinflussen speziell freiwillige, unbezahlte Arbeit, Spenden und Subventionen die innerbetriebliche Lohnstreuung von NPO?

Zur Beantwortung der Fragestellungen wird im Kapitel 10 zuerst ein Überblick über verschiedene Theorien zur Lohnstreuung gegeben, um danach im Kapitel 11 auf Besonderheiten des Nonprofit Sektors in Bezug auf die Lohnstreuung einzugehen. Kapitel 12 fasst vorhandene empirische Untersuchungen zusammen, die im gewinnorientierten Bereich sowie im Nonprofit Sektor Lohnstreuung analysieren. Kapitel 13 gibt einen Überblick über den Modellrahmen, Variablen der Schätzung sowie die erwarteten Vorzeichen. Im Kapitel 14 werden Angaben zur Berechnung gemacht, bevor im Kapitel 15 die Ergebnisse dargestellt und die Forschungsfragen beantwortet werden.

\section{Theorien - Was kann die Lohnstreuung in Organisationen beeinflussen?}

In diesem Kapitel sollen Erklärungsansätze für die innerbetriebliche Lohnstreuung gefunden werden. Obwohl einige der vorgestellten Theorien auch oder zum überwiegenden Teil zur Erklärung der zwischenbetrieblichen Lohnstreuung verwendet werden, können sie auch auf die innerbetriebliche Lohnstreuung Auswirkung haben. Zum Teil werden Theorien, die im Kapitel 3 vorgestellt wurden, herangezogen und überlegt, inwiefern sie auch die innerbetriebliche Lohnstreuung erklären (vgl. Kapitel 10.1, 10.2, 10.4, 10.5, 10.6 und 10.7). Fairness- und Anreiz-modelle (10.3), wiederum beschäftigen sich mit Auswirkungen von Lohnstreuung auf die Produktivität der Arbeitnehmer/-innen.

Die in Kapitel 10 vorgebrachten Ansätze haben für gewinnorientierte und Nonprofit Organisationen Relevanz, Kapitel 11 geht in Folge näher auf Auswirkungen der speziellen Bedingungen von NPO auf die Lohnstreuung ein. 


\subsection{Die Zusammensetzung der Arbeitnehmer/-innen in Organisationen: Humankapitaltheorie und Lohnstreuung}

Die erste und trivialste Erklärung, warum Löhne innerhalb von Firmen bzw. in einer Volkswirtschaft streuen, ist, dass sich Individuen unterscheiden. Individuelle Eigenschaften beeinflussen die individuelle Lohnhöhe, was zu Lohnstreuung führt, wenn Individuen mit unterschiedlichen Eigenschaften in einer Organisation arbeiten. Unterschiedliche Grenzproduktivitäten führen zu Lohndifferenzen (vgl. Fitzenberger, Kohn 2005: 6), die Lohnstreuung in einer Firma spiegelt also zum Teil Unterschiede in der Qualifikation ihrer Beschäftigten wider. Die Lohndifferenziale zwischen den einzelnen Beschäftigungsgruppen ergeben sich aus einer Vielzahl von Merkmalen, einerseits aus qualifikationsbezogenen andererseits aus personen-bezogenen Eigenschaften wie Geschlecht, Alter oder Ethnizität (vgl. Ross 1981: 11). Lohndifferenziale zwischen Beschäftigungsgruppen sollen vor allem Unterschiede in den Fähigkeiten der Arbeitnehmer/innen, der Verantwortung und den Beiträgen zur Gesamtleistung einer Organisation reflektieren. Je höher Qualifikation bewertet wird, desto höher sind Lohnunterschiede in einer Volks-wirtschaft.

Wie auch zur Erklärung der Lohnhöhe kann die Humankapitaltheorie als Ausgangspunkt betrachtet werden (vgl. Mincer 1958). Je heterogener die Zusammensetzung der Belegschaft in einer Organisation ist, desto größer ist die erwartete Lohnstreuung. Wichtige Determinanten, die auf etwaige unterschiedliche Produktivität rückschließen lassen, sind demnach Seniorität und Ausbildung. Alter, Geschlecht oder Nationalität können als Proxys für die erworbene Qualifikation, die Seniorität und/oder die Produktivität dienen. Wenn organisationsspezifisches Humankapital für die Tätigkeit in einer Organisation von groBer Bedeutung ist, dann führt dies dazu, dass Lohnzuwächse aufgrund von Seniorität sehr hoch sind. Die innerbetriebliche Lohnstreuung ist dann groß, wenn Arbeitnehmer/-innen unterschiedlicher Seniorität beschäftigt sind (vgl. Groshen 1991a: 365).

Die unterschiedlichen Löhne, die die verschiedenen Produktivitäten mit sich ziehen, können einerseits extern festgelegt werden. Der Lohn kann z.B. der Marktlohn sein, der sich durch Angebot und Nachfrage bestimmt, oder er kann andererseits durch zentrale Lohnsetzer wie die Regierung oder die Sozialpartner vorgegeben werden (vgl. Lazear, Shaw 2007: 14).

\subsection{Kollektive Lohnverhandlungen und Lohnstreuung - Verhandlungstheorien}

$\mathrm{Zu}$ einem gewissen Teil bestimmt sich die Lohnstreuung in europäischen Volkswirtschaften durch kollektive Lohnverhandlungen und wird nicht ausschließlich durch den Markt bestimmt. In Europa gibt es unterschiedliche Ver- 
fahren der Lohnverhandlungen. Zum Teil werden Kollektivlöhne durch Firmenverträge, Branchenkollektivverträge, regionale oder nationale Verträge festgelegt, die möglicherweise auch nebeneinander und ergänzend bestehen.

Das Modell der Verhandlungstheorien besagt, dass die die Lohnstreuung von der Verhandlungsmacht der kollektiven Arbeitnehmer/-innen und der Arbeitgeber/-innen abhängt. Gewerkschaften versuchen, die Oligopsonmacht von Firmen zu verringern, indem kollektiv verhandelt wird (vgl. Fitzenberger, Kohn 2005: 7). Obwohl auch das Gegenteil denkbar ist, geht man generell von der Hypothese aus, dass Gewerkschaften versuchen, die Lohnspreizung zu verringern. Je höher die Marktmacht der Arbeitnehmer/-innen ist, desto höher ist die Lohnhöhe in einer Organisation ${ }^{51}$ und desto niedriger ist die Lohnstreuung innerhalb der Organisation (vgl. z.B. Metcalf et al. 2000). Gewerkschaften setzen sich für eine egalitärere Lohnstruktur ein. Je höher die Gewerkschaftsmacht, desto größer ist die Stauchung der Lohnverteilung (vgl. Fitzenberger, Kohn 2005: 6). Erklärt werden kann das Phänomen damit, dass risikoaverse Gewerkschaftsmitglieder, für die die Zukunft hinsichtlich ihrer Produktivität bzw. ihrer Einkünfte als unsicher gilt, ex ante gleicher verteile Löhne bevorzugen (vgl. Gerlach, Stephan 2005: 7; Fitzenberger, Kohn 2005: 6).

Freeman (1980: 3f.) führt auch Gründe an, warum eine gegenteilige Hypothese, nämlich dass Gewerkschaften tendenziell die Lohnstreuung vergrößern, denkbar wäre. Wenn Gewerkschaften nur für ihre Mitglieder das Lohnniveau heben, vergrößert sich die Lohnspreizung, wenn nur hoch bezahlte Arbeitnehmer/-innen organisiert sind. Wenn nur schlecht bezahlte Individuen organisiert sind, verkleinert sich die Lohnspreizung.

$\mathrm{Zu}$ einem gewissen Teil wird innerbetriebliche Lohnstreuung auch von Gewerkschaften akzeptiert, da Lohnsteigerungen nach dem Senioritäts-prinzip gefordert werden, d.h. eine mit der Anstellungsdauer bzw. dem Alter steigende Entlohnung, im Vergleich zu einer Entlohnung, die sich an der individuellen Leistung orientiert (vgl. Metcalf et al. 2000: $2 \mathrm{f}$. sowie 16). Gewerkschaften lassen Lohnstreuung nach Seniorität oder Alter auch deshalb zu, weil es für gut verdienende Arbeitnehmer/-innen ex-post sonst nicht mehr rational ist, Gewerkschaftsmitglied zu sein (vgl. Fitzenberger, Kohn 2005: 6). Wenn es möglich ist, würden gut qualifizierte Arbeitnehmer/-innen aus dem System herausoptieren, wenn die Lohnstreuung zu sehr reduziert wird, indem sie entweder individuelle Verträge abschließen oder eigene Gewerkschaften bilden. Firmen, die Kollektivverträgen mit einer geringen Lohnstreuung unterliegen, könnten ihre Lohnschemata ändern, indem sie z.B. Stückraten einführen (vgl. Gerlach, Stephan 2005: 7).

Hirsch (1982) gibt außerdem zu bedenken, dass die Kausalität zwischen Gewerkschaftszugehörigkeit und Lohnstreuung nicht eindeutig ist. So ist es auch 
denkbar, dass Lohnstreuung auf die Gewerkschaftszugehörigkeit wirkt und nicht umgekehrt. Dabei reflektiert die Lohnstreuung die Homogenität der Arbeitnehmer/-innen. Je homogener eine Gruppe ist, desto wahrscheinlicher ist ein gewerkschaftlicher Zusammenschluss, da Interessen und Präferenzen ähnlicher sind, was den Zusammenschluss stabiler macht (vgl. Hirsch 1982: 24f.). Je ähnlicher sich Arbeitnehmer/-innen sind, desto leichter austauschbar sind sie, was wiederum eine Stärkung der Verhandlungsmacht auf Arbeitnehmerseite durch einen gewerkschaftlichen Zusammenschluss verlangt. Auch wird angenommen, dass die Kosten eines Zusammenschlusses für homogene Individuen geringer sind. Andererseits kann gerade eine hohe Lohndispersion den Ruf nach einem gewerkschaftlichen Zusammenschluss seitens der Arbeitnehmer/-innen lauter machen.

Wesentlich können auch institutionelle Faktoren sein, in der Bestimmung des Einflusses kollektiver Lohnverhandlungen auf die Lohnstreuung, wie etwa der Ebene auf der kollektiv verhandelt wird und der Verbindlichkeit von Kollektivverträgen für die Organisationen. So gibt es zum Teil auch zusätzliche Firmenkollektivverträge. Dabei ist es wahrscheinlicher, dass große Firmen eigene Firmenkollektivverträge haben (vgl. Kapitel 10.4). Es ist unklar, ob zusätzliche Verträge auf lokaler Ebene die Lohnstreuung vergrößern oder vermindern (vgl. z.B. Dell'Aringa, Lucifora 1994). In europäischen Volkswirtschaften hat die einzelne Firma als Lohnsetzerin aufgrund zentralisierter Lohn-verhandlungen weniger Handlungsspielraum als etwa in den USA in Bezug auf das Lohnsetzungsverhalten (vgl. Cardoso 1997: 542). Das führt dazu, dass zwischen- und innerbetriebliche Lohnunterschiede weniger stark ausgeprägt sind.

\subsection{Fairness- und Anreizmodelle}

Innerbetriebliche Lohnstreuung kann auch verschiedene Lohnpolitiken der Organisation widerspiegeln. Sie kann bewusst versuchen, den Arbeitnehmer/-innen gleiche Löhne zu zahlen, oder genau das Gegenteil, eine relativ hohe Streuung der Löhne einführen. Beide Fälle können damit erklärt werden, dass sich die Organisationen durch das eingeführte Lohnschema höhere Produktivität ihrer Arbeitnehmer/-innen erhofft. Dahinter liegen zwei gegensätzliche Hypothesen über den Zusammenhang von Produktivität der Individuen und der Lohnstreuung, nämlich die Fair-wage Hypothese und die Tournamententlohnungstheorie.

Zum Teil werden in Organisationen überraschend wenig Unterschiede in der Entlohnung zwischen Individuen gemacht, auch wenn offen-sichtliche Produktivitätsunterschiede bestehen (vgl. Pfeffer, Davis-Blake 1990: 40). Dies kann zum Teil mit der Fair-wage Hypothese erklärt werden. Arbeitnehmer/-innen reduzieren ihre Arbeitsanstrengung, wenn sie sich nicht angemessen entlohnt fühlen 
(vgl. z.B. Charness, Kuhn 2007: 693). Für die Beurteilung über die Angemessenheit des eigenen Lohnes vergleichen sie auch ihren Lohn mit dem ihrer Kolleg/-inn/en. D.h. nicht die absolute Lohnhöhe, sondern die relative ist ausschlaggebend in der Einschätzung, ob sie mit ihrem Lohn zufrieden sind. Die Fair-wage Hypothese unterstellt einen Zusammenhang zwischen empfundener Fairness der Entlohnung und der Arbeitsleistung bzw. der Kooperation zwischen Arbeitnehmer/-innen (vgl. Akerlof, Yellen 1990; Levine 1991, Frank 1984). Im Vergleich des eigenen Lohns mit dem anderer können andere Arbeitnehmer/innen der gleichen Berufsgruppe der eigenen Firma, alle Arbeitnehmer/-innen der eigenen Firma, andere Beschäftigte der gleichen Berufsgruppe generell oder andere Beschäftigte in anderen Firmen generell Bezugsgruppen sein. Nähere Bezugsgruppen haben jedoch im Vergleich größeres Gewicht, sodass besonders die ersten beiden genannten Bezugsgruppen von Bedeutung sind (vgl. Leete 2000: 425). Eine besondere Rolle für den Vergleich spielen die Löhne von Manager/-inne/n, die Arbeitsanstrengung von Arbeitnehmer/-innen hängt im besonderen Maße von deren Löhnen ab (vgl. Hesse, Rivas 2007). Ein Vergleich des wahrgenommenen Arbeitsinputs und -outputs vom Individuum selbst mit dem der Bezugsgruppe führt zur Einschätzung, ob der eigene Lohn als gerechtfertigt angesehen wird oder nicht. Die Einschätzung über die Lohnfairness hat wiederum Auswirkungen auf die intrinsische Motivation und Produktivität der einzelnen Arbeitnehmer/-innen. Intrinsisch motiviert sind Personen dann, wenn die Arbeit um der Arbeit willen verrichtet wird. Hohe innerbetriebliche Lohnstreuung senkt die intrinsische Motivation und den Gruppenzusammenhalt, was wiederum die Produktivität der einzelnen Arbeitnehmer/-innen und die durchschnittliche Firmenleistung schmälert, besonders wenn Kooperation der Einzelnen von Bedeutung ist. Je höher dabei die Mitarbeiterverantwortung bzw. der Ermessensspielraum des oder der Einzelnen ist, desto größer sind derartige Auswirkungen. Personen, die mit ihrem Lohn im Vergleich zu ihren Kolleg/-inn/en unzufrieden sind, versuchen ihre Situation zu ändern, entweder indem sie ihren Arbeitsinput ändern und sich weniger kooperativ zeigen oder indem sie langfristig das Unternehmen verlassen. Eine weitere Möglichkeit zur „Lösung“ der als unfair empfundenen Situation ist die Anpassung der Einschätzung über das Verhältnis des eigenen Arbeitsinputs und -outputs zu dem der Vergleichsperson, um so zu einem gerechten Empfinden zu kommen.

Als Begründung für dieses Verhalten kann auch der, Gift Exchange'-Ansatz ${ }^{52}$ herangezogen werden, der besagt, dass Arbeitnehmer/-innen in dem Ausmaß etwas zurückgeben, in dem sie etwas empfangen haben, d.h. dass sie ihre Leistung an die empfundene Gerechtigkeit über den eigenen Lohn anpassen (vgl. Akerlof 1982). Organisationen komprimieren aus diesem Grund die Lohnstreuung. Arbeitgeber/-innen bevorzugen eine geringe Lohnstreuung auch aus dem 
Grund, da so individuelle Lohnforderungen einzelner, besser qualifizierter Arbeitnehmer/-innen auf ein Minimum reduziert werden können (vgl. Machin, Manning 2004: 384).

Lazear (1991: S.95) hält dem entgegen, dass ein Verlust von Motivation und darauffolgender Produktivitätsnachlass einzelner Arbeitnehmer-gruppen nicht notwendigerweise zu einem Produktivitätsverlust für das Unternehmen führt. Gewisse Einkommensgruppen im Unternehmen profitieren ja von einer größeren Lohnstreuung, was wiederum zu einem Produktivitätsgewinn bei diesen führen kann. Andererseits wird bezweifelt, ob ein Lohngewinn im gleichen Ausmaß zu Änderungen in der Motivation, d.h. vermehrten Anstrengungen, führt wie ein Verlust an Lohn (vgl. Charness, Kuhn 2007; Leete 2000: 426). Charness und Kuhn (2007) gehen von zwei verschiedenen Modellen aus, einem symmetrischen und einem asymmetrischen. Im ersten Fall reagieren Arbeitnehmer/-innen in gleichem Maße auf (verhältnismäßige) „Über-“ und „Unterbezahlung“. Im asymmetrischen Fall verhalten sich Arbeitnehmer/-innen nur bei „Unterbezahlung“ anders, nicht aber bei „Überbezahlung“. Die Autoren zeigen, dass in beiden Modellfällen eine Verringerung der Lohnstreuung für Unternehmen gewinnmaximierend sein kann, wenn Arbeitnehmer/-innen auf Löhne der Kolleg/inn/en reagieren (vgl. Charness, Kuhn 2007: 713).

Inwieweit der Lohnvergleich zwischen Arbeitnehmer/-inne/n eine Rolle spielt hängt auch davon ab, inwiefern Löhne anderer Mitarbeiter/-innen bekannt sind. Je weniger Löhne bekannt sind, desto weniger wichtig ist es in Hinsicht auf die Fair-wage Hypothese, eine geringe Lohnstreuung zu haben. Dementsprechend müsste geringeres Wissen über die Löhne der Kolleg/-inn/en zu einer höheren Lohnstreuung führen (vgl. Pfeffer, Davis-Blake 1990: 41, Charness, Kuhn 2007; Hesse, Rivas 2007).

Anreizmodelle zum anderen beruhen auf der Vorstellung, dass eine möglichst große Lohnstreuung produktivitätssteigernd wirkt, und stehen damit im direkten Gegensatz zu Fairness-Modellen. Der Tournamententlohnungstheorie zufolge konkurrieren Arbeitnehmer/-innen innerhalb einer Firma untereinander, der „Sieger" erhält eine Beförderung und/oder höhere Entlohnung (vgl. Lazear, Rosen 1981). Eine größere Lohnstreuung soll die Konkurrenz unter den Arbeitnehmer/-innen verstärken (vgl. Franz 2006: 324), sodass die Lohnstreuung auch die Streuung der Grenzprodukte übersteigen kann (vgl. Machin, Manning 2004: 381). Nicht die individuelle Leistung per se, sondern die Leistung im Vergleich $\mathrm{zu}$ anderen wird beurteilt, womit die Firma eine kostspielige Bewertung der individuellen Leistung vermeiden kann. Große Lohnstreuung, damit auch hohe „Preise“ für „Sieger" am oberen Ende der Lohnstreuung, soll die motiviertesten und qualifiziertesten Arbeitnehmer/-innen anziehen, was sich durch hohe Produktivität dieser Arbeitskräfte wiederum positiv auf die Firmenleistung auswirkt. Je mehr „Teilnehmer/-innen“ am „Lohnturnier“ mitmachen, desto größer ist die Konkurrenz unter den Arbeitnehmer/-innen und desto höher die erwartete 
Lohnstreuung (vgl. Heyman 2005: 1315). Für Firmen kann ein großer Wettbewerb unter Arbeitnehmer/-innen dann zum Problem werden, wenn sie untereinander nicht mehr kooperieren und sich gegenseitig sabotieren, um die Arbeitsleistung anderer zu reduzieren, womit die Wirksamkeit derartiger „Turniere“ für Firmen eingeschränkt wird (vgl. z.B. Franz 2006: 324).

\subsection{Spezialisierung, Firmengröße und Lohnstreuung}

Der Zusammenhang zwischen der Firmengröße und der Lohnhöhe ist ein oft untersuchter Forschungsgegenstand. Etwas seltener wird auch untersucht, ob sich große und kleine Unternehmen in ihrer Lohnstreuung unterscheiden. Es sprechen einige Argumente für eine positive Korrelation von Lohnstreuung und Firmengröße, einige dagegen:

Für höhere Lohnstreuung bei höherer Firmengröße spricht einerseits die Vermutung, dass größere Firmen bezüglich ihrer Qualifikation heterogenere Arbeitnehmer/-innen beschäftigen. Hohe Divergenz führt zu einer größeren Lohnstreuung (vgl. Haltiwanger, Davis 1996: 330f.). Unterschiede zwischen großen und kleinen Firmen können auf unterschiedliche Produktionstechniken zurückgeführt werden. Große Firmen sind sowohl vertikal als auch horizontal differenzierter, was dazu führt, dass eine höhere Vielfalt an Arbeitsaufgaben besteht, wofür sie unterschiedlich qualifizierte Arbeitnehmer/-innen benötigen. Es wird weiters vermutet, dass gut ausgebildete Individuen größere Fähigkeiten haben, sich Renten zu sichern, da sie über höhere Verhandlungsmacht verfügen. Deshalb wird davon ausgegangen, dass das Vorhandensein sehr unterschiedlichen Humankapitals in Unternehmen - über den Weg verschiedener Fähigkeiten Renten abzuziehen - zu höherer Lohnstreuung führt (vgl. Haltiwanger, Davis 1996).

Andererseits verwenden größere Firmen häufiger leistungsabhängige Entlohnungsstrukturen (vgl. Lazear, Rosen 1981). Je mehr Mitarbeiter/-innen in einer Firma, desto größer die Lohnspreizung, um die Produktivität der Arbeitnehmer/innen zu erhöhen.

Für eine geringere Lohnstreuung bei höherer Firmengröße spricht die Hypothese, dass größere Firmen nicht heterogenere Arbeitnehmer/-innen, sondern genau umgekehrt - homogenere beschäftigen. Demnach verwenden größere Firmen standardisierte Produktions-techniken, woraus folgt, dass sich auch die Arbeitnehmer/-innen in ihren Fähigkeiten ähnlicher sind (vgl. Haltiwanger, Davis 1996: 330f.). Weiters kann argumentiert werden, dass größere Firmen nach festen Lohnschemen bezahlen, um geringere Kosten zur Feststellung der Leistung des einzelnen Individuums zu haben (vgl. Lallemand, Rycx 2006: 310). Ein weiteres Argument ist, dass größere Firmen häufiger kollektivvertraglichen Regelungen unterliegen oder einen größeren Anteil an Arbeitnehmer/- 
innen mit Gewerkschaftszugehörigkeit haben. Allgemein wird angenommen, dass Kollektivverträge und Gewerk-schaften die Lohnspreizung vermindern (vgl. z.B.: Freeman 1982) (vgl. Kapitel 10.2).

Obwohl Argumente für und gegen einen positiven Zusammenhang zwischen Organisationsgröße und höherer Lohnstreuung vorliegen, wird in empirischen Arbeiten überwiegend ein positiver Zusammenhang gefunden, wie in Kapitel 12 noch näher ausgeführt wird.

\subsection{Rent-Sharing, Effizienzlöhne und kompensierende Lohnzahlungen}

Die Rent-Sharing Theorie, die Effizienzlohntheorie sowie die Theorie der kompensierenden Lohnzahlungen erklären, warum Löhne in einem Unternehmen über (bzw. im Fall der kompensierenden Lohnzahlungen auch unter) Marktlöhnen liegen können, was im Kapitel 3 schon ausführlich erläutert wurde.

Hinsichtlich der innerbetrieblichen Lohnstreuung stellt sich nun die Frage, ob alle Individuen innerhalb einer Organisation gleich stark von den in den Theorien vorgestellten Ideen betroffen sind. So kommt es gemäß der Rent-Sharing Theorie dann zu einer Änderung der innerbetrieblichen Lohnstreuung, wenn die Renten nicht anteilsmäßg auf alle Arbeitnehmer/-innen ausbezahlt werden.

Effizienzlöhne liegen in jenen Branchen besonders weit über dem Markträumungslohn, in denen hohe Überwachungskosten und eine hohe Arbeitnehmerfluktuation vorherrschen. Die Höhe der Überwachungs-kosten und die Arbeitnehmerfluktuation ist vermutlich nicht bei allen Arbeitnehmer/-inne/n innerhalb einer Organisation gleich. Deshalb stellt sich die Frage, ob in Folge nur bestimmte Arbeitnehmer/-innen Effizienzlöhne erhalten (vgl. u.a. Landmann, Jerger 1999: 183ff.; Levine 1991). Die Theorie der relativen Position geht davon aus, dass Normen über Gerechtigskeitsvorstellungen eine große Bedeutung für die Arbeitnehmer/-innen haben, wodurch es sinnvoll sein kann, auch jenen Personen innerhalb einer Organisation Effizienzlöhne zu zahlen, die keine hohen Überwachungskosten oder keine hohe Arbeitnehmer-fluktuation haben, was für eine kleine Lohnstreuung spricht (vgl. Gerlach, Stephan 2006b: 48f.). ${ }^{53}$ Durch eine Ausdehnung von Zahlungen aufgrund von Rent-Sharing oder Effizienzlöhnen auch auf Lohngruppen in einer Firma, wo die Zahlung nicht notwendig sind, soll der Gruppenzusammenhalt gestärkt werden, was in Folge wiederum zu einer Produktivitätssteigerung führen soll (vgl. Levine 1991). ${ }^{54}$

53 Vgl. dazu Kapitel 10.3.

54 Dabei wird unterstellt, dass die Fair-Wage Hypothese zutrifft (vgl. Kapitel 10.3). 
Im Fall der kompensierenden Lohnzahlungen vergrößert sich dann die Lohnstreuung, wenn bestimmte Arbeitsbedingungen nicht für alle Stellen innerhalb des Unternehmens zutreffen (vgl. Groshen 1991a: 367).

Es stellt sich in Folge die Frage, ob besondere Bestimmungsgründe feststellbar sind, die entscheiden, ob nur bestimmte Arbeitnehmer/-innen in einer Organisation höhere Löhne aufgrund von Renten, Effizienzlöhnen oder kompensierenden Lohnzahlungen erhalten oder ob das Unternehmen diese Lohnpolitik auf alle Arbeitnehmer/-innen in der Organisation ausdehnt. Einerseits kann es sein, dass in unterschiedlichen Unternehmen oder Branchen ein unterschiedlich hoher Prozentsatz der Arbeitnehmer/-innen z.B. von schlechteren Arbeitsbedingungen betroffen ist, weshalb kompensierende Löhne gezahlt werden. Zwei Unternehmen könnten sich demnach in ihrer Lohnstreuung unterscheiden, wenn sehr viele oder sehr wenige Arbeitnehmer/-innen davon betroffen sind. Andererseits kann dabei auf die Literatur bezüglich der Fairness- und Anreiz-Hypothesen zurückgegriffen werden (vgl. Kapitel 10.3). Wie in Kapitel 12.1.3 und 12.2 noch dargestellt werden wird, liegen unterschiedliche empirische Ergebnisse vor, welche der beiden Hypothesen zutreffender ist, und es zeigt sich, dass in bestimmten Sektoren eher der Fairness-Ansatz überwiegt. Möglicherweise beeinflusst auch die institutionelle Anbieterform, welcher der beiden Ansätze vorzuziehen ist. Wenn der Fairness-Ansatz überwiegt, d.h. wenn niedrigere Lohnstreuung zu höherer Produktivität der Arbeitnehmer/-innen führt, dann ist auch anzunehmen, dass Renten, Effizienzlöhne und kompensierende Lohnzahlungen eher an die gesamte Belegschaft einer Organisation ausgedehnt werden. In bestimmten Branchen oder institutionelle Anbieterformen könnte auch eine besondere Organisationskultur vorherrschen, die, unabhängig von den Folgen für die Produktivität, eine höhere Lohngleichheit bevorzugt, sodass derartige Zahlungen auf alle Arbeitnehmer/-innen ausgedehnt werden.

Ein weiterer Indikator dafür könnte die Verhandlungsmacht der Arbeitnehmer/innen sein. Je höher diese ist, desto eher könnten Zahlungen über dem Markträumungsniveau an alle Arbeitnehmer/-innen ausgedehnt werden. Die Existenz von Betriebsräten könnte beispielsweise ein Hinweis dafür sein.

\subsection{Such- und Matching Modelle}

Lohnstreuung beruht bei Such- und Matching Modellen nicht auf unterschiedlichen Grenzproduktivitäten der Arbeitnehmer/-innen. Diese Theorien erklären Lohndispersion unter ansonsten identischen Individuen aufgrund unvollständiger Informationen im Suchprozess der Individuen. Diese Modelle sind zuerst eher dazu geeignet, zwischenbetriebliche Lohnstreuung zu erklären, weshalb hier nur am Rande auf diese Modelle eingegangen wird (vgl. z.B. auch Groshen 1991a; Mortensen, Pissarides 1999). Durch die unvollständige Informa- 
tion der Individuen entstehen Suchfriktionen und matchspezifische Renten. Die Arbeitnehmer/-innen sind beim Suchprozess nicht vollständig informiert und akzeptieren in Folge Lohnangebote von Firmen, die unterhalb ihrer Grenzproduktivität liegen (vgl. Fitzenberger, Kohn 2005: 6).

Innerbetriebliche Lohnstreuung kann bei Such- und Matchingmodellen durch Fluktuationskosten erklärt werden, die entstehen, wenn ein Individuum seinen Arbeitgeber wechselt. Entstehen Firmen nämlich Kosten, wenn Arbeitnehmer/innen ihren Arbeitsplatz wechseln, sind sie an einer möglichst geringen Arbeitnehmerfluktuation interessiert. Wenn Arbeitnehmer/-innen bei anderen Firmen ein höherer Lohn geboten wird, bieten Firmen diesen Personen denselben Lohn, damit sie in der Firma bleiben. Dies führt zu Lohnstreuung zwischen Individuen innerhalb einer Firma, auch wenn diese dieselbe Produktivität haben. Langfristig führt das jedoch auch dazu, dass Firmen, deren Arbeitnehmer/-innen viele Angebote von außen bekommen im Durchschnitt höhere Löhne zahlen. Will eine Organisation dies vermeiden, wird sie selten auf Lohnangebote von außen mit gleich hohen Löhnen eingehen und die innerbetriebliche Lohnstreuung relativ gering halten (vgl. Machin, Manning 2004: 383).

\subsection{Sorting-by-abilities Theorie}

Die Sorting-by-abilities Theorie, die zumeist auf Kremer und Maskin (1996) zurückgeführt wird, geht davon aus, dass sich Arbeitnehmer/-innen unterschiedlicher Produktivität in Firmen sortieren, d.h. es gibt Firmen, die ausschließlich Arbeitnehmer/-innen hoher Produktivität beschäftigen, Firmen, die einzig Personen mittlerer Produktivität und welche, die nur Individuen mit niedriger Produktivität beschäftigen. Es ist nicht unmittelbar einleuchtend, wieso es dazu kommen soll. Wenn nämlich alle Arbeitnehmer/-innen nach ihrem Grenzprodukt bezahlt werden, so müsste es einer Firma egal sein, ob sie zwei Individuen mit gleicher Produktivität zu einem bestimmten Lohnsatz beschäftigt oder eine Person mit doppelter Produktivität mit doppeltem Lohn (vgl. Groshen 1991a: 363). Ein Grund, warum die Sortierung in Firmen dennoch stattfindet, ist, dass durch die verwendete Technologie eine bestimmte Qualifikation bzw. Produktivität notwendig wird. Möglich ist auch, dass die Produktivität eines Individuums von der eines anderen abhängt, d.h. alle Arbeitnehmer/-innen können nur so produktiv sein wie das Individuum mit der geringsten Produktivität. Aus diesem Grund sollen nur Individuen mit der gleichen Produktivität in einem Team arbeiten. Findet ein solcher Sortierungsprozess zwischen Firmen statt, so geht die Streuung des Qualifikationsniveaus innerhalb einer Organisation zurück. Einhergehend mit dieser Entwicklung ist ein Anstieg der Lohnungleichheiten zwischen Organisationen. Diese Theorie erklärt, warum manche Organisationen ein höheres Lohnniveau haben als andere. Gleichzeitig heißt das, dass die innerbetriebliche Lohnstreuung dadurch niedrig ist (vgl. Kremer, Maskin 1996). 


\subsection{Zusammenfassung}

Zusammenfassend lässt sich festhalten, dass innerbetriebliche Lohnstreuung zum einen Produktivitätsunterschiede der einzelnen Arbeitnehmer/-innen ausdrücken sollen. Wie die einzelnen Produkt-ivitätsunterschiede entlohnt werden, kann am Markt durch Angebot und Nachfrage, aufgrund von kollektiven Verhandlungen durch Gewerkschaften und Arbeitgebervertreter/-innen auf übergeordneter Ebene oder aufgrund von Firmenlohnpolitik bestimmt werden. Theoretisch lassen sich Argumente finden, warum Gewerkschaften für oder gegen eine niedrige Lohnstreuung votieren, es überwiegt jedoch in der vorhandenen empirischen und theoretischen Literatur die Meinung, dass gewerkschaftliches Engagement meist zu einer geringeren Lohnstreuung führt. Zum anderen ist Lohnstreuung das Ergebnis von Firmenlohnpolitik. Hier sind zwei gegensätzliche Konzepte zu nennen - die Anschauung, dass Lohnkompression zu einer höheren Produktivität Einzelner führt und die dazu im Gegensatz stehende Auffassung, dass hohe Lohnunterschiede den Wettbewerb unter den Individuen verstärken und damit produktivitätssteigernd wirken.

Über den Zusammenhang zwischen der Firmengröße und der Lohnstreuung lassen sich ebenso Argumente für eine positive wie negative Korrelation finden, wobei in der vorhandenen Literatur zumeist von einem positiven Zusammenhang ausgegangen wird. Rent-Sharing, Effizienzlöhne und kompensierende Lohnzahlungen können die Lohnstreuung in einer Firma beeinflussen, je nachdem ob derartige Zahlungen an alle Arbeitnehmer/-innen der Firma getätigt werden oder nur an einen Teil. Wenn sich Arbeitnehmer/-innen gleicher Qualifikation zunehmend nach Firmen sortieren, wie in der Sorting-by-abilities Theorie unterstellt, kommt es zunehmend zu einem Rückgang der innerbetrieblichen Lohnstreuung und einer Zunahme der zwischen-betrieblichen. Bei Such- und Matching Modellen wird innerbetriebliche Lohnstreuung auf Fluktuationskosten zurückgeführt, die Arbeitgeber/-inne/n entstehen. Um derartige Kosten zu vermeiden, gehen Arbeitgeber/-innen auf Lohnforderungen von Arbeitnehmer/inne/n ein, wodurch im Laufe der Zeit Lohnstreuung auch unter gleich Qualifizierten entstehen kann.

\section{Besonderheiten von NPO in Bezug auf die Lohnstreuung}

Davon ausgehend, dass bei NPO in Bezug auf die Lohnsetzung Besonderheiten vorliegen $^{55}$, kann darauf geschlossen werden, dass auch in Bezug auf die Lohnstreuung bei NPO Unterschiede zu gewinn-orientierten Unternehmen bestehen. Leete (2000) geht von der Hypothese aus, dass Unterschiede in der Mission, 
eine besondere Motivation der Arbeitnehmer/-innen und die im Vergleich zu gewinnorientierten Unternehmen Erstellung anderer Güter und Dienstleistungen die Lohnstreuung einer NPO beeinflussen. Eine hohe intrinsische Motivation der Arbeitnehmer/-innen in NPO würde faire Löhne erfordern, d.h. Gleichheit in der Entlohnung hat in NPO besonderen Stellenwert. Dabei unterstellt Leete, dass sich eine (zu) hohe Lohnstreuung negativ auf die intrinsische Motivation der Arbeitnehmer/-innen auswirkt.

Dieses Kapitel geht auf Besonderheiten von NPO ein, die die Lohnstreuung beeinflussen können, wobei insbesondere auf die Faktoren der Forschungsfrage Ehrenamt, Spenden und Subventionen - eingegangen wird. Das Kapitel 11.1 greift Unterschiede bezüglich der Lohnstreuung zwischen gewinnorientierten Firmen und NPO auf, wobei im Kapitel 11.1.1 auf Fairness- und Anreiztheorien aus dem vorherigen Kapitel 10.3 zurückgegriffen wird. Das Kapitel 11.1.2 geht auf Löhne von Führungskräften in NPO ein, da die hohen Löhne in einer Organisation die innerbetriebliche Lohnstreuung besonders beeinflussen. Während die beiden erwähnten Unterkapitel mögliche Unterschiede in der Lohnstreuung zwischen gewinnorientierten Firmen und NPO erklären, geht das Kapitel 11.2 darauf ein, warum es Unterschiede zwischen NPO gibt. Darin wird überlegt, wie sich nonprofit-spezifischen Faktoren auf die Lohnstreuung auswirken.

\subsection{Unterschiede bezüglich der Lohnstreuung zwischen dem gewinnorientierten und dem Nonprofit Sektor}

\subsubsection{Fairness und Anreize in NPO}

Die Organisationsziele und Werthaltungen einer NPO können auch die innerbetriebliche Lohnstreuung beeinflussen. Die Lohnstreuung innerhalb des Nonprofit Sektors und innerhalb einer NPO ist ein bislang kaum untersuchtes Thema. Womöglich sind auch hier fehlende Daten als Grund zu nennen.

Bezüglich der beiden konkurrierenden Theorien, der Fair-wage Hypothese und der Tournamententlohnungstheorie wird vermutet, dass im Nonprofit Sektor, die Fair-wage Hypothese im Vordergrund steht. Leete (2000) stellt in ihrer Arbeit die Hypothese auf, dass sich die vermutete höhere intrinsische Motivation von Mitarbeiter/-innen in NPO nicht nur auf die Lohnhöhe auswirkt (vgl. Hansmann 1980), sondern auch auf die Lohnspreizung innerhalb der Organisation. NPO sind besonders auf Arbeitnehmer/-innen mit hoher intrinsischer Motivation angewiesen. Leete führt dies im Zusammenhang mit den Existenz-gründen von NPO an (vgl. 2000: 427f.). NPO bestehen zum einen aufgrund von Informationsasymmetrien, zum anderen um besondere Ansichtsweisen von Interessensgruppen aufrechtzuerhalten. In beiden Fällen sind hoch intrinsisch motivierte Arbeitnehmer/-innen vonnöten, vor allem im Bereich des 
Managements. NPO müssen Wege finden, um die hohe intrinsische Motivation aufrecht zu erhalten, wobei hier auf eine geringe innerbetriebliche Lohnspreizung besonderen Wert gelegt wird, da davon ausgegangen wird, dass die intrinsische Motivation bzw. die Arbeitsmoral sinkt, wenn sich Arbeitnehmer/innen unfair oder ungleich behandelt oder der jeweiligen Gruppe nicht zugehörig fühlen (Leete 2000, 425). Folglich ist davon auszugehen, dass in NPO vorwiegend der Fair-wage Hypothese der Vorzug gegeben wird. Eine geringe Lohnspreizung innerhalb einer Organisation, d.h. eine relativ gleiche Lohnverteilung, soll die intrinsische Motivation und die Identifikation mit einer Gruppe stärken.

Am Rande sei auch erwähnt, dass geringe Lohnstreuung auch deshalb in NPO vorherrschend sein kann, da weniger Diskriminierung betrieben wird und aus diesem Grund gleichere Löhne gezahlt werden (vgl. dazu auch Kapitel 12.2).

Gegen das Vorliegen einer Tournamententlohnung spricht auch die Vermutung, dass NPO vorwiegend in Branchen mit flachen Hierarchien tätig sind, etwa in der Kinder- und Altenbetreuung oder in sozialen Diensten. Flache Hierarchien deuten auf eine geringe innerbetriebliche Lohnstreuung (vgl. Devaro, Brookshire 2007: 317). Die Möglichkeit einer Beförderung in NPO ist, einer empirischen Untersuchung mit US-amerikanischer Daten zufolge, signifikant weniger oft gegeben ist. Im Vergleich zu gewinnorientierten Unternehmen ist nicht nur die Möglichkeit einer Beförderung seltener, die Wahrscheinlichkeit, innerhalb der Organisation tatsächlich befördert zu werden, ist ebenso geringer. Allerdings ist dieser Unterschied vorwiegend für qualifizierte Arbeitnehmer/innen beobachtbar, bei unqualifizierten Individuen ist der Unterschied zwischen den beiden Sektoren weniger starkt ausgeprägt (vgl. Devaro, Brookshire 2007). Wenn es zu Beförderungen in NPO kommt, dann basieren diese weniger auf individiuellen Leistungen, sondern eher auf Seniorität oder positiver Diskriminierung. Lohnerhöhungen, die aus Beförderungen resultieren, sind in NPO und gewinnorientierten Unternehmen jedoch ca. gleich hoch (Devaro, Brookshire 2007).

Insgesamt zeigt sich, dass der Zusammenhang zwischen der individuellen Leistung und dem Lohn in NPO insgesamt weniger stark ausgeprägt ist. So erhalten Individuen im Nonprofit Sektor weniger oft Sonderzahlungen oder Boni (vgl. Devaro, Brookshire 2007: 329, Roomkin, Weisbrod 1999). Als Grund dafür wird die hohe intrinische Motivation und die Angst vor einem Crowdingout Effekt der intrinsischen Motivation genannt, wenn ein leistungsabhängiges Lohnschema eingeführt werden würde. Auch die Tatsache, dass in NPO weniger Hierarchiestufen existieren und außerdem weniger Beförderungen und weniger leistungsabhängige Zahlungen erfolgen, deutet darauf hin, dass die Tournamententlohnungstheorie in NPO weniger Relevanz hat, und statt dessen dem Fairness-Ansatz Vorzug gegeben wird, woraus eine geringere Lohnstreuung im Vergleich zu anderen institutionellen Formen resultiert. 


\subsubsection{Löhne von Führungskräften in NPO}

Besondere Beachtung in der Forschung zu Löhnen im Nonprofit Sektor finden Löhne von Führungskräften. Sie wurden in dieser Dissertation bereits im ersten Teil thematisiert, als Unterschiede zwischen Lohnquantilen herausgearbeitet wurden. Auch für die innerbetriebliche Lohnstreuung sind sie von großer Bedeutung, insofern als sie als extreme Ausreißer die innerbetriebliche Lohnstreuung maßgeblich beeinflussen können und sie auch als bedeutende Vergleichswerte in Bezug auf die Einschätzung über die Fairness der Entlohnung der Arbeitnehmer/-innen dienen. Niedrige Löhne von Führungskräften könnte den Arbeitnehmer/-innen einer Organisation etwa flache Hierarchie, Teamgeist oder Solidarität signalisieren. Die Thematik der Entlohnung der Führungskräfte hat für NPO große Relevanz, da sie mit der Legitimationsproblematik von NPO eng verbunden ist (vgl. z.B. Gray, Benson 2003; Hallock 2002; Leete 2006; Oster 1998; Roomkin, Weisbrod 1999). So haben Löhne von Führungskräften in NPO besondere Bedeutung in der Frage der Finanzierung der Organisation. Aufgrund des erschwerten Zugangs zu Krediten vieler NPOs, haben andere externe Finanzierungsmöglichkeiten wie z.B. private Spender/-innen, Unternehmen, Stiftungen und staatliche Institutionen besondere Bedeutung (vgl. z.B. Littich 2007: $333 \mathrm{ff}$.). Hohe Managerlöhne in NPO können bei hoher Abhängigkeit von Spenden Signalwirkung haben und werden von der allgemeinen Öffentlichkeit mitunter kritisch und als Verschwendung von Spenden oder Subventionen interpretiert (vgl. Roomkin, Weisbrod 1999: 751). ${ }^{56}$ Sowohl Klient/-inne/n als auch Spender/-innen der NPO kann die Entlohnung der Führungskräfte interessieren (vgl. Oster 1998: 208). Niedrige Managerlöhne sollen als produktives Zeichen für uninformierte Konsument/-inn/en dienen, d.h. wiederum dass der Fokus der Organisation bei der Mission liegt und Spenden nicht „verschwendet" werden (vgl. Hallock 2002: 382).

Andererseits kann das Argument vorgebracht werden, dass hohe Gehälter mit hoher Qualität der Güter und Dienstleistungen verbunden sind und hohe Löhne damit gute Qualität signalisieren. All dies setzt natürlich wiederum voraus, dass die Löhne der Öffentlichkeit auch bekannt sind.

Der erschwerte Zugang zu externen Finanzierungsquellen für NPO kann auch ein Grund sein, warum in NPO niedrigere Löhne gezahlt werden, da für höhere Löhne keine ausreichenden Ressourcen vorhanden sind. Es steht hierbei also weniger das Argument der Signalwirkung gegenüber Arbeitnehmer/-innen und der interessierten Öffentlichkeit im Vorder-grund, sondern die mangelnde Zahlungsfähigkeit. Aus diesem Grund könnte auch vermutet werden, dass es dadurch zu einer Selektion der Führungskräfte zwischen gewinnorientiertem Sek-

Vgl. dazu Kapitel 4.6. 
tor und Nonprofit Sektor kommt und folglich vermehrt Führungskräfte mit geringerer Leistungsfähigkeit im Nonprofit Sektor arbeiten.

Wenn Führungskräfte in NPO niedrigere Löhne erhalten als ihre Pendants im gewinnorientierten Bereich, ist die Lohnspreizung bei ansonsten gleichen Löhnen innerhalb von NPO geringer.

\subsection{Unterschiede bezüglich der Lohnstreuung zwischen Nonprofit Organisationen}

Während im Kapitel 11.1 Überlegungen angestellt wurden, warum Unterschiede zwischen NPO und gewinnorientierten Unternehmen hinsichtlich der Lohnstreuung bestehen, soll nun geklärt werden, ob es diese auch zwischen NPO gibt. Im zweiten Teil der Dissertation soll die Forschungsfrage beantwortet, ob und wie Zuwendungen in Form von ehrenamtlichen Mitarbeiter/-inne/n, Spenden und Subventionen die innerbetriebliche Lohnstreuung von NPO beeinflussen. Dieser Abschnitt soll Argumente bringen, warum diese die Lohnstreuung beeinflussen können. Hierfür wird auf Theorien und Argumente aus den Kapiteln 10 und 11.1 zurückgegriffen.

Ehrenamtliche Mitarbeiter/-innen können auf eine flache Hierarchie der (bezahlten) Arbeitskräfte deuten. Hier kommt es wiederum darauf an, welche Positionen bezahlte und welche Positionen unbezahlte Arbeitskräfte innerhalb der NPO einnehmen. Werden nur wenige Positionen mit bezahlten Arbeitnehmer/innen besetzt, so deutet das auf eine geringe Lohnstreuung, da durch die freiwilligen Mitarbeiter/-innen bestimmte Lohnsegmente zur Gänze wegfallen können, sodass die Lohnstreuung womöglich gering ist. Es stellt sich, wie im ersten Teil der Arbeit, die Frage, welche Tätigkeiten die freiwilligen Mitarbeiter/-innen verrichten. Sowohl Tätigkeiten am oberen wie am unteren Ende des Lohnsegments, also Tätigkeiten in der Führung der Organisation und Hilfsdienste, verringern die Lohnstreuung, wenn durch freiwillige Arbeit Lohnsegmente wegfallen.

Ehrenamtliche Mitarbeiter/-innen können wiederum ein Indikator dafür sein, inwiefern das Gut oder die Dienstleistung der NPO als sozial erwünscht erachtet wird bzw. dafür, ob die Mission der Organisation als wichtig eingestuft wird und inwiefern sich die Arbeitnehmer/-innen in Folge durch hohe intrinsische Motivation auszeichnen. Hohe intrinsische Motivation der Mitarbeiter/-innen deutet auf eine niedrige Lohnstreuung, da besonders Führungskräfte in dem Fall bereit sein könnten, auf einen Teil des Lohns zu verzichten bzw. für einen geringen Lohn zu arbeiten, was in Folge die Lohnstreuung verkleinert. Besonders Führungskräfte können dieser Überlegung nach, die sozialen Auswirkungen der Tätigkeiten der NPO einschätzen, weshalb diese Gruppe an Arbeitnehmer/-innen bereit sein könnte, auf einen Teil des Lohns zu verzichten (siehe dazu auch Kapitel 4.5). 
Im ersten Teil der Dissertation wurde gezeigt, dass die oberen Lohngruppen mehr von höheren Spenden und Subventionen profitieren als die unteren. Dies kann als ein Hinweis verstanden werden, dass Renten nicht an alle Mitarbeiter/innen einer Organisation ausgedehnt werden, sondern nur bestimmten Gruppen zukommt (vgl. auch Kapitel 10.5). Wenn sich also höhere Spenden und Subventionen positiv auf die Lohnhöhe der oberen Einkommen auswirken, so kann eine hohe Abhängigkeit von Spenden und Subventionen die Lohnstreuung vergrößern. Mehr Geld durch diese Einkommensarten bietet den Organisationen die Möglichkeit, Löhne stärker zu variieren, sodass sich eine hohe Abhängigkeit dieser Einkommensarten positiv auf die Lohnstreuung auswirkt.

Hohe Abhängigkeit von öffentlichen Geldern wie Spenden oder Subventionen kann jedoch darauf hindeuten, dass die NPO allgemein von der öffentlichen Wahrnehmung abhängig ist und damit die Legitimationsproblematik von NPO für diese Organisationen besonders akut ist. Eine zu hohe Lohnstreuung bzw. sehr hohe Löhne von Führungskräften können die Legitimität nach außen gefährden (vgl. dazu auch Kapitel 11.1.2). Das setzt wiederum voraus, dass der Öffentlichkeit bzw. den Spender/-inne/n oder Subventionsgeber/-inne/n das Lohnschema oder die Löhne der Führungskräfte auch bekannt sind. Insgesamt ist diese Problematik für Spenden aber höher einzuschätzen als für Subventionen.

In Bezug auf Subventionen wäre es auch möglich, dass Lohnniveaus wiederum durch den Geldgeber bzw. die Geldgeberin vorgeschrieben oder an öffentliche Lohnschemen angelehnt sind. Dies hätte für die Lohnstreuung zur Folge, dass sie für die Organisation nicht frei variabel ist. $\mathrm{Ob}$ sich ein derartiges Verhalten positiv oder negativ auf die Lohnstreuung auswirkt, hängt davon ab, wie sich NPO hinsichtlich der Lohnstreuung von öffentlichen Unternehmen unterscheiden. Wenn diese ohne Einflussnahme von außen niedriger wäre, würde sich eine hohe Abhängigkeit von Spenden demnach positiv auf die Lohnstreuung auswirken. Die Argumentation in einfachen Worten wäre, dass NPOs durch eine hohe Subventionsabhängigkeit größere Lohnunterschiede machen müssen als sie wollen. Es ist jedoch auch die umgekehrte Argumentation denkbar.

Zusammenfassend lässt sich festhalten, dass ehrenamtliche Arbeit vermutlich zu einer geringeren Lohnstreuung führt. Einerseits fehlen durch ehrenamtliche Arbeit womöglich Lohnsegmente. Wenn etwa freiwillige Mitarbeiter/-innen Hilfsdienste oder Führungsarbeit leisten, so verringert das die Lohnstreuung. Andererseits deuten ehrenamtliche Mitarbeiter/-innen womöglich auf eine hohe intrinsische Motivation, da die Mission der NPO als wichtig eingestuft wird, was sich möglicherweise negativ auf die Lohnstreuung auswirkt. Der Effekt einer höheren Abhängigkeit von Spenden und Subventionen bleibt unklar, da sowohl Argumenten für einen positiven als auch einen negativen Einfluss sprechen. Hohe Lohngruppen profitieren mehr von hohen Spenden und Subventionen, d.h. von Rent-Sharing, was die Lohnstreuung vergrößern kann. Außerdem 
wäre es möglich, dass im Fall der Subventionen durch eine Einflussnahme von außen, ein Lohnschema vorgegeben ist, das die Lohnstreuung vergrößert. Umgekehrt trifft die Legitimationsproblematik nach außen besonders bei Organisationen zu, die eine hohe Abhängigkeit von Spender/-innen und Subventionsgeber/-innen aufweisen, was zu niedrigeren Löhnen besonders bei Führungskräften führen könnte. Dies würde auf ein negatives Vorzeichen dieser Variablen deuten.

\section{Vorhandene empirische Evidenz}

In diesem Kapitel werden relevante vorhandene empirische Analysen zu Determinanten der innerbetrieblichen Lohnstreuung vorgestellt. In Kapitel 12.1 wird auf Ergebnisse im gewinnorientierten Bereich eingegangen, Kapitel 12.2 präsentiert empirische Studien, die Relevanz für den Nonprofit Sektor haben. Außerdem werden in Kapitel 12.3 empirische Ergebnisse zur Lohnhöhe von Führungskräften im Nonprofit Sektor vorgestellt. Sowohl Kapitel 12.2 als auch Kapitel 12.3 vergleichen zumeist NPO mit gewinnorientierten Firmen. Eine Untersuchung, die sich mit Unterschieden zwischen NPO beschäftigt, gibt es meines Wissens nach nicht.

Aufgrund begrenzter Datenverfügbarkeit beschränken sich ältere Untersuchungen oft auf Lohnstreuung innerhalb und zwischen Branchen (vgl. z.B. Abowd, Kramarz 2000; Barsky, Personick 1981; Dickens et al. 1987; Groshen 1991b; Hartog et al. 2000; Hirsch 1982; Kahn 1998). Erst durch das Vorhandensein von Arbeitnehmerdaten mit den dazugehörigen Informationen zu deren Arbeitgebern wurde der Untersuchungsbereich auch auf die Lohnstreuung in und zwischen Firmen ausgedehnt.

\subsection{Innerbetriebliche Lohnstreuung im gewinnorientierten Bereich}

Die vorhandene Literatur zur innerbetrieblichen Lohnstreuung im gewinnorientierten Bereich kann im Großen und Ganzen in drei Kategorien eingeteilt werden. Die erste beschäftigt sich mit dem Zusammenhang zwischen der Organisationsgröße und der Lohnstreuung, die zweite untersucht den gewerkschaftlichen Einfluss bzw. die Auswirkungen von Kollektivverträgen auf die innerbetriebliche Lohnstreuung. Die dritte Kategorie überprüft, ob der Fairness-Ansatz oder die Tournamententlohnungstheorie empirisch nachweisbar sind.

Weitere vorhandene Studien, die nicht in eine der drei oben genannten Kategorien fallen, befassen sich entweder mit anderen Determinanten der innerbetrieblichen Lohnstreuung (wie z.B. dem Alter der Organisation) oder beschreiben die Veränderung der innerbetrieblichen Lohnstreuung im Zeitverlauf, stellen aber 
eher einer Minderheit dar (vgl. James, Morlock 1987; Nordström Skans et al. 2007; Davis, Haltiwanger 1991; Pfeffer, Davis-Blake 1990).

International gesehen finden Lazear und Shaw (2007: 6) überraschend wenig Unterschiede zwischen verschiedenen westeuropäischen Ländern und den USA, was die Lohnstreuung betrifft.

\subsubsection{Firmengröße und Lohnstreuung}

Ein erster Literaturstrang behandelt den Zusammenhang zwischen Firmengröße und der Lohnstreuung. (vgl. z.B.: Davis, Haltiwanger 1995; Haltiwanger, Davis 1996; Lallemand, Rycx 2006). Generell zeigt sich empirisch, dass sich die Organisationsgröße positiv auf die innerbetriebliche Lohnstreuung auswirkt, d.h. dass größere Firmen eine größere Lohnstreuung haben.

Davis und Haltiwanger (1995) bzw. Haltiwanger und Davis (1996) untersuchen den Zusammenhang als erste umfassend mit US-amerikanischen Daten aus dem produzierenden Gewerbe aus dem Jahr 1982. Dabei finden sie, dass die innerbetriebliche Lohnstreuung mit der Firmengröße steigt. Der Grund dafür wird in der größeren Heterogenität der Arbeitnehmer/-innen mit zunehmender Firmengröße gesehen. Keinen Hinweis finden sie für die Richtigkeit der Hypothese, dass große Unternehmen mehr leistungsorientierte Bezahlungsschemen haben (Davis, Haltiwanger 1995: 33f). Lallemand und Rycx (2006) untersuchen denselben Zusammenhang für vier europäische Länder - Belgien, Italien, Irland und Spanien - anhand von LEE-Daten. Auch sie finden einen positiven Zusammenhang zwischen Firmengröße und Lohnstreuung aufgrund heterogenerer Arbeitnehmer/-innen (vgl. Lallemand, Rycx 2006: 323). Kleine Unternehmen orientieren ihre Lohnsetzung häufiger an der individuellen Leistung des Einzelnen bzw. der Einzelnen, während größere Firmen standardisierte Lohnschemen haben.

\subsubsection{Verhandlungsmacht und innerbetriebliche Lohnstreuung}

Die zweite Kategorie befasst sich mit dem Zusammenhang zwischen kollektiven Lohnverhandlungen auf verschiedenen Ebenen und den Auswirkungen auf die Lohnstreuung innerhalb eines Unternehmens. Dazu werden auch Untersuchungen gezählt, die sich mit Gewerkschaftszugehörigkeit von Arbeitnehmer/-innen und Lohn-streuung beschäftigen. Zu dieser Kategorie ist eine Vielzahl von Untersuchungen in verschiedenen Ländern vorhanden (vgl. z.B.: für Nordamerika: Freeman 1980; Freeman 1982; und für Europa: Bechtel et al. 2005; Canal Dominguez, Rodriguez Gutierrez 2004; Dell'Aringa, Lucifora 1994; Gerlach, Stephan 2005; Gerlach, Stephan 2006a; Gosling, Machin 1995; Metcalf et al. 2000; Rodriguez Gutierrez 2001).

Zum einen wird z.B. auf einem Makro-Level die Korrelation zwischen Zentralisierungsgrad von Lohnverhandlungen und der Einkommens-ungleichheit in ver- 
schiedenen Ländern analysiert (vgl. z.B.: OECD 1997: Kapitel 3, OECD 2004: Kapitel 3). Generell wird angenommen, dass zentralisiertere Lohnverhandlungen zu egalitäreren Löhnen führen (vgl. z.B. Dell'Aringa, Lucifora 1994: 152).

Auf Mikroebene wird ein möglicher Zusammenhang zwischen der innerbetrieblichen und zwischenbetrieblichen Lohnstreuung und Lohnverhandlungen, die auf verschiedenen Ebenen durchgeführt werden können, untersucht.

Weitgehend kommen die Forscher/-innen in den Studien zum Schluss, dass Gewerkschaften tendenziell zu einer geringeren Lohnstreuung führen und dass dieser Effekt dann größer ist, wenn die Gewerkschaftszugehörigkeit bzw. der Anteil der Arbeitnehmer/-innen, deren Löhne durch zentrale Lohnverhandlungen festgelegt werden, groß ist. Außerdem führen zentrale Lohnverhandlungen zu einer geringeren Lohnstreuung als dezentrale (vgl. Dell'Aringa, Pagani 2007: 30).

Einige Forscher/-innen beschäftigen sich mit der Frage, ob unterschiedliche Verträge, z.B. Branchen- oder Firmentarifverträge, zu unterschiedlichen Ergebnissen führen. So verwenden Gerlach und Stephan (2005 und 2006a) deutsche Lohnstrukturdaten, um zu untersuchen, ob sich die inner- und zwischenbetriebliche Lohnstreuung unter Vorhandensein von Kollektivverträgen auf Branchenund Firmenebene und bei Firmen ohne Kollektivverträge für Arbeiter/-innen unterscheiden. Sie zeigen, dass die innerbetriebliche Lohnstreuung in Firmen ohne Kollektivvertrag größer ist, dass sich allerdings Firmen mit Branchen- und Firmenvertrag in Bezug auf die innerbetriebliche Lohnspreizung kaum voneinander unterscheiden. Eine ähnliche Forschungsfrage verfolgen Bechtel, Heinbach und Strotmann (2005). Ebenfalls anhand deutscher Daten untersuchen sie, wie sich Tarifbindung auf die Lohnhöhe und innerbetriebliche Lohnstreuung auswirkt. Ein statistisch signifikanter Einfluss von Tarifbindung auf die Lohnstreuung konnte nur bei Arbeiter/-innen gefunden werden, nicht aber bei Angestellten. Für Arbeiter/-innen jedoch fällt das Urteil ähnlich aus wie bei Gerlach und Stephan, nämlich dass die Betriebe, die tariflich gebunden sind, eine geringere Lohnstreuung haben. Ein weiteres Ergebnis der Studie ist, dass mit zunehmender Streuung des Alters in der Belegschaft auch die Lohnstreuung im Betrieb zunimmt, die Betriebsgröße hat hingegen keinen Einfluss auf die Lohnstreuung (vgl. Bechtel et al. 2005: 171).

Canal Dominguez und Rodriguez Gutierrez (2004) konzentrieren sich auf die Unterschiede zwischen Firmen- und Branchenverträgen, wobei sie zu dem Ergebnis kommen, dass Unternehmen mit Firmen-kollektivverträgen eine höhere innerbetriebliche Lohnstreuung aufweisen als jene, die einen Branchenvertrag haben.

Andere Forscher/-innen untersuchen, ob sich unterschiedliche Anteile von Gewerkschaftsmitgliedern an allen Arbeitnehmer/-innen zu unterschiedlichen Auswirkungen führen. Dell'Aringa und Lucifora (1994) finden mit italienischen 
Daten Organisationsdaten aus dem Bereich der Metallerzeugung und dem Maschinenbau aus dem Jahr 1990 einen durch gewerkschaftlichen Einfluss verringerte Lohnspreizung. Gewerkschaften haben dann mehr Einfluss, wenn mehr Arbeitnehmer/-innen eines Unternehmens Gewerkschaftsmitglieder sind. Lohndifferenzen aufgrund unterschiedlicher Qualifikation werden verringert, d.h. schlecht Ausgebildete haben bei höherer Gewerkschafts-zugehörigket der Arbeitnehmer/-innen ceteris paribus höhere Löhne, besser Ausgebildete niedrigere.

Gosling und Machin (1995) verwenden britische Daten auf Organisationsebene, um die innerbetriebliche Lohnstreuung zu messen. Bei der Befragung wurde gefragt, wie viele Arbeitnehmer/-innen weniger als 50\% des durchschnittlichen Arbeitnehmers bzw. der durchschnittlichen Arbeitnehmerin verdienen, wie viele über $200 \%$ des durchschnittlichen Arbeitnehmers bzw. der durchschnittlichen Arbeitnehmerin. Aus den Antworten ergibt sich, dass Firmen, in denen Gewerkschaften agieren, auch nach Kontrolle anderer Einflussfaktoren weniger häufig Mitarbeiter/-innen mit einem Lohn beschäftigen, der weniger als $50 \%$ oder mehr als 200\% des Lohns eines durchschnittlichen Arbeitnehmers bzw. einer durchschnittlichen Arbeitnehmerin beträgt (vgl. Gosling, Machin 1995: 174f.). Auch Metcalf, Hansen und Charlwood (2000) zeigen mit britischen Daten, dass Gewerkschaften die Lohnstreuung reduzieren, indem sie standardisierte Lohnschemen bevorzugen und die Divergenzen zwischen Geschlechtern, Arbeiter/innen und Angestellten oder zwischen verschiedenen Nationalitäten reduzieren (vgl. Metcalf et al. 2000: 16).

Freeman (1980) zeigt für die USA, dass ein hoher Organisationsgrad der Arbeitnehmer/-innen die Lohnspreizung in einer Firma reduziert. Dabei wird vor allem die Lohndifferenz zwischen Arbeiter/-inne/n und Angestellten reduziert. In einem weiteren Artikel befasst sich Freeman (1982) ausführlicher mit derselben Fragestellung und stellt auch hier fest, dass Firmen, deren Arbeitnehmer/-innen $\mathrm{zu}$ einem hohen Anteil einer Gewerkschaft angehören, eine kleinere Lohnspreizung haben. Diese Firmen wenden häufiger standardisierte Lohnschemen an als Betriebe, in denen Arbeitnehmer/-innen nicht gewerkschaftlich organisiert sind.

\subsubsection{Fairness und Anreize}

Bei der dritten Kategorie werden die Tournamententlohnungstheorie und die Fair-wage Hypothese getestet, d.h. der Zusammenhang zwischen der innerbetrieblichen Lohnstreuung und der Produktivität eines Indi-viduums bzw. der Produktivität einer Organisation untersucht (vgl. z.B.: Beaumont, Harris 2003; Cowherd, Levine 1992; Heyman 2002; Heyman 2005; Lallemand et al. 2004 ; Lallemand et al. 2007b; Levine 1991; Winter-Ebmer, Zweimüller 1999). Hier steht die Lohnstreuung als erklärende Variable auf der rechten Seite der Regressionsgleichung. In der Gesamtbeurteilung der Studien fällt auf, dass die Ergebnisse zu unterschiedlichen Schlüssen kommen, wobei vermehrt der Tournamententlohnungstheorie der Vorzug gegeben wird. 
Winter-Ebmer und Zweimüller (1999) verwenden österreichische ArbeitnehmerArbeitgeber Daten. Ein niedriges Level an Lohnspreizung ist bei Angestellten für die Leistung und Produktivität des Unternehmens förderlich, eine zu hohe Lohnspreizung bewirkt jedoch das Gegenteil und ist hinderlich. Bei Arbeitern bzw. Arbeiterinnen ist eine inner-betriebliche Lohnspreizung produktivitätsfördernd (vgl. Winter-Ebmer, Zweimüller 1999: 569f.).

Lallemand, Plasman und Rycx (2004 und 2007b) finden mit belgischen Arbeitnehmer-Arbeitgeberdaten ebenso eher Bestätigung für das Tournamententlohnungsmodell, d.h. einen positiven Zusammenhang zwischen innerbetrieblicher Lohnspreizung und Produktivität des Unternehmens (vgl. Lallemand et al. 2004: 553). Dies gilt besonders für Arbeiter/-innen und in Betrieben mit hoher Überwachung der Arbeitnehmer/-innen. Allerdings untersuchen sie nur Firmen mit mehr als 200 Mitarbeiter/-innen.

Auch Jirjahn und Kraft (2007) zeigen für die deutsche Fertigungsindustrie, dass der Zusammenhang zwischen Lohnstreuung und Produktivität schwach positiv ist. Stückraten verstärken den Effekt, interne Beförderungen als Anreizmechanismus in einer Firma schwächen den Effekt.

Beaumont und Harris (2003) untersuchen für fünf Industriezweige in Großbritannien diesen Zusammenhang und finden keine allgemein gültige Korrelation zwischen Lohnstreuung und Organisationsleistung. Für vier Sektoren ist die Produktivität bei höherer Lohnspreizung größer, in der pharmazeutischen Industrie führt geringere Lohnspreizung zu höherer Produktivität. Außerdem gilt, dass bei kleinen, heimischen Unternehmen eher geringere Lohnspreizung zu höherer Produktivität führt, weniger stark ist dieser Zusammenhang bei großen Firmen in ausländischem Besitz (vgl. Beaumont, Harris 2003: 60f.).

Heymann (2005) prüft ebenfalls mit einem LEE-Datensatz aus Schweden die Tournamententlohnungstheorie für Angestellte und Führungskräfte. Er findet einen positiven Zusammenhang zwischen Lohnstreuung und Firmenleistung. Die Lohnspreizung nimmt bei höherer Anzahl von Manager/-inne/n ab, was der Tournament-entlohnungstheorie widerspricht (vgl. Heyman 2005: 1323f). Ebenfalls schwedische Daten verwenden Hibbs Jr. und Locking (2000). Auch sie finden keine Bestätigung für das Fairness-Modell, sondern zeigen vielmehr, dass sich eine geringe innerbetriebliche Lohnstreuung negativ auf die Produktivität auswirkt (vgl. Hibbs Jr., Locking 2000: 776f.).

Am Rande sei eine Untersuchung von Cowherd und Levine (1992) erwähnt. Sie zeigen, dass höhere Lohngleichheit im Unternehmen die Produktqualität erhöht.

Insgesamt zeigt sich, dass der Zusammenhang je nach Branche und Tätigkeit der Arbeitnehmer/-innen unterschiedlich ist. 


\subsection{Lohnspreizung in NPO und nonprofit-nahen Branchen}

Die Lohnspreizung innerhalb einer NPO war bislang kaum Forschungsgegenstand. Eine der wenigen Arbeiten dazu stammt von Leete (2000), die anhand von US-amerikanischen Daten aus dem Jahre 1990 die Lohnspreizung im Nonprofit Sektor im Vergleich zum gewinnorientierten Sektor untersuchte. Dazu wurden Individualdaten von Arbeitnehmer/-innen aus allen Branchen des gewinnorientierten und des Nonprofit Sektors verwendet. Demnach ist die Lohnspreizung im NPO Bereich geringer als im Bereich der gewinnorientierten Unternehmen. Da jedoch keine Arbeitnehmer-Arbeitgeber-Daten vorliegen, kann nicht zwischen inner- und zwischenbetrieblicher Lohnstreuung unterschieden werden. Leete unterstellt, dass die Lohnspreizung in NPO aufgrund höherer Motivation der Arbeitnehmer/-innen geringer sein müsste (Leete 2000). Da NPO besonders von der intrinsischen Motivation ihrer Arbeitnehmer/-innen abhängen, hätte die innerbetriebliche Lohnspreizung in diesen Organisationen geringere oder negative Produktivitätswirkung und müsste daher nur im besonders geringen Ausmaß in NPO zu beobachten sein (Leete 2000: 424 und 429). Für die Berechnung der Lohnstreuung zieht sie eine Lohnfunktion heran, die getrennt für die beiden Sektoren geschätzt wird. Dann vergleicht sie die Varianz der Residuen, wobei die Residuen als Differenz zwischen tatsächlichen und geschätzten Löhnen berechnet werden, und führt eine Oaxaca-Blinder Dekomposition $^{57}$ durch. Die Varianz der tatsächlichen Löhne ist im gewinnorientierten Bereich höher als im Nonprofit Sektor (vgl. Leete 2000: 433). Allerdings muss festgehalten werden, dass Leete wohl aufgrund der Datenlage nichts über die Lohnspreizung innerhalb einer NPO aussagen kann und in ihren Ergebnissen auf Sektoren- und Berufsgruppenebene beschränkt bleibt. Determinanten, die auf Organisationsebene liegen, gehen, mit Ausnahme der Sektorzugehörigkeit, nicht als Schätzvariable in das Modell ein. Am größten ist der Unterschied zwischen dem gewinnorientierten und dem Nonprofit Sektor für Fach- und Führungskräfte (vgl.Leete 2000: 442). Die gleichere Lohnverteilung im Nonprofit Sektor führt sie zum Teil auf die geringere Diskriminierung aufgrund von Geschlecht und Herkunft zurück, wobei dieses Ergebnis nur für Angestellte gilt.

Machin und Manning (2004) untersuchen die Lohnstreuung von Pflegehelfer/innen in englischen Pflegeheimen und finden nur eine sehr geringe innerbetriebliche Lohnstreuung in diesem Bereich. Circa ein Drittel der Heime im Datensatz zahlen allen Pflegehelfer/-innen genau denselben Lohn, ein weiteres Drittel zahlt nur zwei verschiedene Löhne. Sie erklären die Tatsache mit der Präferenz der Arbeitnehmer/-innen nach einer geringen Lohnstreuung, d.h. mit der Fairnesshypothese. Obwohl interessanterweise der Pflegebereich typischerweise aus öf- 
fentlichen, gewinnorientierten und Nonprofit Einrichtungen besteht, unterscheiden die Autoren nicht nach den drei Sektoren. In diesem Fall kann also keine Aussage darüber getroffen werden, ob die niedrige innerbetriebliche Lohnstreuung im Bereich der Pflegeheime z.B. aus der Tatsache zu erklären ist, dass sich eventuell viele Nonprofit Heime im Datensatz befinden oder ob dies z.B. eine Eigenart des Pflegesektors ist.

Pfeffer und Langton (1993) untersuchen anhand von US-amerikanischen Universitätsangestellten den Zusammenhang zwischen Lohnstreuung auf der einen Seite und Arbeitszufriedenheit, Forschungsproduktivität und Zusammenarbeit mit Kolleg/-inn/en auf der anderen Seite und finden Bestätigung für die Fair-wage Hypothese. Auch sie diskutieren nicht näher, ob es sich hierbei um Angestellte des Nonprofit Sektors, des gewinnorientierten oder des öffentlichen Sektors handelt. Je höher die Lohnspreizung, desto höher ist die Unzufriedenheit mit der Arbeit, desto niedriger ist die Forschungsleistung und desto geringer ist die Wahrscheinlichkeit, mit anderen in der Forschung zusammenzuarbeiten. Reaktionen auf die Lohnstreuung hängen jedoch davon ab, inwieweit Personen über die Lohnhöhe anderer Mitarbeiter/-innen Bescheid wissen, sowie von der eigenen Stellung in der Lohnstruktur (vgl. Pfeffer, Langton 1993: 403).

Anhand von Löhnen in amerikanischen Baseball Teams wird der Zusammenhang zwischen Lohnspreizung und Teamwork untersucht (vgl. Depken II 2000). Der Autor findet Unterstützung für die Fair-wage Hypothese. Höhere Lohnspreizung senkt die Teamleistung. Auch hier wird keine Aussage darüber getroffen, ob es sich bei den Baseball-Teams um NPO handelt. Zumindest kann aber davon ausgegangen werden, dass es sich bei Sportteams um einen für NPO relevanten Tätigkeitsbereich handelt.

Eine Studie, die sich konkret mit innerbetrieblicher Lohnspreizung im Nonprofit Sektor befasst, existiert meines Wissens nach nicht.

\subsection{Löhne von Führungskräften im Nonprofit Sektor}

Es liegen einige Studien vor, die sich mit Determinanten von Löhnen von Führungskräften im Nonprofit Sektor befassen. Themen von Interesse sind dabei, inwiefern Manager/-innen leistungsabhängig bezahlt werden oder der Zusammenhang zwischen der Lohnhöhe von Führungskräften und der Organisationsgröße, sowie der Einfluss verschiedener Finanzierungsstrukturen auf die Lohnhöhe. Auswirkungen der Lohnhöhe von Manager/-innen auf die inner- oder zwischenbetriebliche Lohnstreuung sind zwar nicht Forschungs-gegenstand in diesen Studien, doch sind entsprechende Schluss-folgerungen möglich. Wie schon in Kapitel 11.1.2 angedeutet beeinflussen Löhne von Führungskräften als Ausreißer nach oben die Lohnstreuung im besonderen Maß, außerdem stellen 
sie einen bedeutenden Vergleichswert in Bezug auf die Einschätzung über die Fairness der Entlohnung der Arbeitnehmer/-innen dar.

Nach einer Untersuchung von Roomkin und Weisbrod (1999), bei der Managergehälter in amerikanischen Nonprofit und For-profit Krankenhäusern verglichen wurden, zeigt sich, dass leistungsabhängige Zahlungen an Führungskräfte vermehrt in gewinnorientierten Einrichtungen vorgenommen werden. Das Grundgehalt im Nonprofit Sektor ist höher als im gewinnorientierten Bereich, jedoch sind durch geringe zusätzliche Boni wenig Steigerungen möglich, sodass insgesamt Führungskräfte in gewinnorientierten Krankenhäusern um ca. 25\% mehr verdienen. Es wird erwartet, dass die unterschiedlichen Entlohnungssysteme verschiedene Managertypen anziehen - im Nonprofit Sektor befinden sich vermehrt risikoscheue Individuen. Die Stellen beinhalten der Analyse zufolge jedoch die gleichen Aufgaben und Ver-antwortungen, sodass der Bezahlungsunterschied nicht auf Unterschiede in den Stellen zurückgeführt werden kann.

Ballou und Weisbrod (2003) beschäftigen sich ebenfalls mit Managerlöhnen in Krankenhäusern und unterscheiden zusätzlich innerhalb des Nonprofit Sektors zwischen kirchlichen und nicht-kirchlichen Krankenhäusern. Kirchliche Einrichtungen setzen vermehrt auf hohe Grundlöhne mit niedrigeren leistungsabhängigen Zahlungen (vgl. Ballou, Weisbrod 2003: 1908ff.). Auch bei der Untersuchung von Frumkin und Keating (2001) zeigt sich, dass leistungsorientierte Bezahlung bei Führungskräften in NPO weniger stark verbreitet ist. Als Grund wird die Angst der NPO vermutet, dass leistungsabhängige Lohnelemente das Gewinnausschüttungsverbot von NPO verletzen.

In empirischen Untersuchungen zeigt sich auch, dass die Lohnhöhe von Führungskräften stark mit der Organisationsgröße korreliert. Oster (1998) findet mit US-amerikanischen Daten über NPO aus ver-schiedenen Bereichen, dass mit zunehmender Organisationsgröße die Löhne der Führungskräfte steigen. Allerdings ist der Effekt weniger stark ausgeprägt als in gewinnorientierten Unternehmen. Hallock (2002) verwendet US-amerikanische Paneldaten, um Einflussfaktoren auf Löhne von Führungskräften zu analysieren. Auch er findet einen positiven Zusammenhang zwischen der Organisationsgröße einer NPO und der Lohnhöhe von Manager/-inne/n, der genauso, wie in der Untersuchung von Oster (1998) etwas geringer ausfällt als in gewinnorientierten Unternehmen. Auch Frumkin und Keating (2001: 20) können diesen Zusammenhang mit USamerikanischen Daten bestätigen. Gray und Benson (2003) analysieren Determinanten von Löhnen von Führungskräften in NPO und zeigen, dass die Organisationsgröße sowie Erfahrung und Ausbildung des Individuums positiv mit der Lohnhöhe korrelieren. Männer verdienen mehr als Frauen, die Organisations- 
leistung - als Kundenzufriedenheit gemessen ${ }^{58}$ - hingegen hat keinen signifikanten Einfluss auf die Lohnhöhe, wenn die Leistung jedoch als Ressourceneffizienz gemessen wird, dann hat sie einen schwach positiven Einfluss (vgl. Gray, Benson 2003: 222).

Oster (1998) nimmt ein Maß für die Abhängigkeit der Organisation von Spendengeldern in die Analyse auf. In den Ergebnissen zeigt sich, dass Führungskräfte um ca. 1.000 US-\$ weniger verdienen, wenn der Anteil der Spenden an den Gesamteinnahmen um einen Prozentpunkt steigt. Im Unterschied zu Oster (1998) untersucht Hallock (2002) nicht den Zusammenhang zwischen Spenden und Löhnen, sondern jenen zwischen öffentlichen Subventionen und der Entlohnung von Führungskräften. Hier findet er im Querschnitt einen positiven Zusammenhang. Organisationen mit öffentlichen Subventionen zahlen um ca. $17 \%$ höhere Löhne an Manager/-innen, die Höhe der Subventionen ist jedoch nicht relevant. Bei Verwendung der Paneldaten und fixen Firmeneffekten wird der Zusammenhang jedoch insignifikant.

\subsection{Zusammenfassung}

Zusammenfassend zeigt sich, dass sich vorhandene empirische Literatur im Bereich der gewinnorientierten Firmen, auf drei Themengebiete konzentriert: Der Einfluss der Firmengröße und der Verhandlungsmacht bei kollektiven Lohnverhandlungen auf die innerbetriebliche Lohnstreuung und die Auswirkungen der Lohnstreuung auf die Produktivität des Unternehmens. Während die Ergebnisse der ersten beiden Kategorien in eine Richtung weisen, sind die Ergebnisse der dritten gemischt: Empirisch zeigt sich, dass größere Firmen eine höhere Lohnstreuung aufweisen und dass das Vorhandensein eines Kollektivvertrags bzw. ein höherer Anteil an Gewerkschafts-zugehörigkeit durch die Arbeitnehmer/innen die innerbetriebliche Lohnspreizung verringert. Bei Untersuchungen, ob eher Fairnessmodelle oder die Tournamententlohnungstheorien als zutreffender einzuschätzen sind, sind die Ergebnisse gemischt. Es liegen sowohl Studien vor, die der Tournamententlohnungstheorie den Vorzug geben, als auch solche, die den Fairness-Ansatz als zutreffender ansehen. Entscheidend ist vermutlich die Branche und die Art der Tätigkeit der Arbeitnehmer/-innen, in der Frage, ob sich eine hohe Lohnstreuung produktivitäts-fördernd oder -hemmend auswirkt.

Betrachtet man die nonprofit-spezifische Literatur, zeigt sich, dass keine Studie vorhanden ist, die sich mit der innerbetrieblichen Lohnstreuung im Speziellen

58 Es wurde dabei angenommen, dass die Organisationen selbst Erhebungen zur Zufriedenheit ihrer Kund/-inn/en durchführen und daher über diese Bescheid wissen. Die von den Organisationen angegebenen Werte wurden schließlich standardisiert (vgl. Gray, Benson 2003: 218f.). 
auseinandersetzt. Bei einer Betrachtung auf Branchenebene oder innerhalb von Berufsgruppen zeigt sich, dass die Lohnstreuung im Nonprofit Sektor geringer ist als im gewinnorientierten Bereich.

Schließlich wurde ein Überblick über Analysen zur Lohnhöhe von Führungskräften in NPO gegeben, da die Löhne von Führungskräften maßgeblich für die Lohnstreuung eingeschätzt werden. Niedrige Löhne von Manager/-inne/n führen zu einer niedrigen Lohnstreuung. Einerseits stellen sie Ausreißer in der Streuung nach oben dar, andererseits fungieren Löhne von Führungskräften oft als Vergleichswerte für Arbeitnehmer/-innen, wenn es um eine Einschätzung der Gerechtigkeit der eigenen Bezahlung geht. Es zeigt sich, dass leistungsorientierte Bezahlung in NPO eine weniger große Rolle spielt, dass die Lohnhöhe von Führungskräften in NPO generell niedriger ist als jene von Manager/-inne/n in gewinnorientierten Unternehmen und dass sich Spenden eher negativ, Subventionen hingegen positiv auf die Lohnhöhe von Führungskräften auswirken.

Folgende zwei Tabellen fassen abschließend die wichtigsten Ergebnisse des Literaturüberblicks im Bereich gewinnorientierter Unternehmen und des Nonprofit Sektors zusammen: 
gewinnorientierte Branchen

\begin{tabular}{|c|c|c|c|c|c|c|}
\hline Autor/-in & Jahr & Thema & Daten & Branche & relevante Variable & Ergebnisse \\
\hline $\begin{array}{l}\text { Davis. } \\
\text { Haltiwanger }\end{array}$ & $\begin{array}{l}1995 \\
1996\end{array}$ & Firmengröße & $\begin{array}{l}\text { USA 1982/83, LEE- } \\
\text { Daten }\end{array}$ & $\begin{array}{l}\text { produzierendes } \\
\text { Gewerbe }\end{array}$ & $\begin{array}{l}\text { Lohnperzentile. Humankapital, } \\
\text { Gewerkschaftszugehørigkeit, } \\
\text { Organisationsgrðße }\end{array}$ & $\begin{array}{l}\text { Firmengröße pos. Einfluss auf } \\
\text { Lohnstreuung }\end{array}$ \\
\hline $\begin{array}{l}\text { Lallemand, } \\
\text { Rycx }\end{array}$ & 2006 & Firmengröße & $\begin{array}{l}\text { Belgien. Italien, Irland. } \\
\text { Spanien 1995, LEE- } \\
\text { Daten }\end{array}$ & verschiedene & $\begin{array}{l}\text { Varianz d. Lohns, Lohnperzentile, } \\
\text { Humankapital, Branche, Kollektivvertrag. } \\
\text { Arbeitsbedingungen }\end{array}$ & $\begin{array}{l}\text { Firmengröße pos. Einfluss auf } \\
\text { Lohnstreuung }\end{array}$ \\
\hline $\begin{array}{l}\text { Gerlach. } \\
\text { Stephan }\end{array}$ & $\begin{array}{l}2005 \\
2006\end{array}$ & $\begin{array}{l}\text { Verhandlungs- } \\
\text { macht }\end{array}$ & $\begin{array}{l}\text { Deutschland 1990/95. } \\
\text { 2001, LEE-Daten }\end{array}$ & verschiedene & $\begin{array}{l}\text { Standardabweichung Lohn, } \\
\text { Kollektivvertrag. Humankapital. Branche, } \\
\text { Organisationsgröße }\end{array}$ & $\begin{array}{l}\text { Lohnstreuung ist in Branchen ohne KV } \\
\text { großer }\end{array}$ \\
\hline $\begin{array}{l}\text { Bechtel, } \\
\text { Heinbach. } \\
\text { Strotmann }\end{array}$ & 2005 & $\begin{array}{l}\text { Verhandlungs- } \\
\text { macht }\end{array}$ & Deutschland & $\begin{array}{l}\text { produzierendes } \\
\text { Gewerbe }\end{array}$ & $\begin{array}{l}\text { Standardabweichung Lohn, Humankapital, } \\
\text { Organisationsgroßße, Kollektivvertrag }\end{array}$ & $\begin{array}{l}\text { bei Arbeiter/-inne/n ist Lohnstreuung in } \\
\text { Betrieben mit Tarifvertrăgen kleiner }\end{array}$ \\
\hline $\begin{array}{l}\text { Canal } \\
\text { Dominguez. } \\
\text { Rodriguez } \\
\text { Gutierrez } \\
\end{array}$ & 2004 & $\begin{array}{l}\text { Verhandlungs- } \\
\text { macht }\end{array}$ & $\begin{array}{l}\text { Spanien 1995. LEE- } \\
\text { Daten }\end{array}$ & verschiedene & $\begin{array}{l}\text { Standardabweichung Lohn. Humankapital. } \\
\text { Organisationsgroße. Kollektivvertrag }\end{array}$ & $\begin{array}{l}\text { Branchenkollektivvertrăge führen zu } \\
\text { niedrigerer Lohnstreuung }\end{array}$ \\
\hline $\begin{array}{l}\text { Dell'Aringa. } \\
\text { Lucifora }\end{array}$ & 1994 & $\begin{array}{l}\text { Verhandlungs- } \\
\text { macht }\end{array}$ & $\begin{array}{l}\text { Italien 1990, Daten auf } \\
\text { Stellenebene }\end{array}$ & $\begin{array}{l}\text { Metall-erzeugung. } \\
\text { Maschinenbau }\end{array}$ & $\begin{array}{l}\text { Standardabweichung Lohn, } \\
\text { Organisationsgroße, Region, Qualifikation, } \\
\text { Gewerkschaftszugehörigkeit }\end{array}$ & $\begin{array}{l}\text { Gewerkschaftlicher Einfluss verringert } \\
\text { Lohnstreuung }\end{array}$ \\
\hline $\begin{array}{l}\text { Gosling. } \\
\text { Machin }\end{array}$ & 1995 & $\begin{array}{l}\text { Verhandlungs- } \\
\text { macht }\end{array}$ & $\begin{array}{l}\text { GB } 1980-90, \\
\text { Organisationsdaten }\end{array}$ & verschiedene & $\begin{array}{l}\text { Standardabweichung Lohn, Humankapital, } \\
\text { gewerkschaftliches Engagement, Anz. AN, } \\
\text { Branche, Arbeitslosenrate }\end{array}$ & $\begin{array}{l}\text { Lohnstreuung ist in Branchen mit } \\
\text { Gewerkschaften niedriger }\end{array}$ \\
\hline Freeman & 1980 & $\begin{array}{l}\text { Verhandlungs- } \\
\text { macht }\end{array}$ & $\begin{array}{l}\text { USA 1973-75, } \\
\text { Individualdaten }\end{array}$ & verschiedene & $\begin{array}{l}\text { Standardardabweichung Lohn, } \\
\text { Gewerkschaftszugehörigkeit, } \\
\text { Humankapital, Region, Stelle }\end{array}$ & $\begin{array}{l}\text { Hoher Organisationsgrad reduziert } \\
\text { Lohnstreuung }\end{array}$ \\
\hline Freeman & 1982 & $\begin{array}{l}\text { Verhandlungs- } \\
\text { macht }\end{array}$ & $\begin{array}{l}\text { USA, Jahr unklar, LEE- } \\
\text { Daten }\end{array}$ & $\begin{array}{l}\text { produzierendes } \\
\text { Gewerbe }\end{array}$ & $\begin{array}{l}\text { Varianz Lohn, Gewerkschaftszugehörigkeit, } \\
\text { Stelle, Branche }\end{array}$ & $\begin{array}{l}\text { Hoher Organisationsgrad reduziert } \\
\text { Lohnstreuung }\end{array}$ \\
\hline $\begin{array}{l}\text { Winter-Ebmer, } \\
\text { Zweimuller }\end{array}$ & 1999 & Produktivităt & $\begin{array}{l}\text { Osterreich 1975-1991, } \\
\text { LEE-Paneldaten }\end{array}$ & verschiedene & $\begin{array}{l}\text { Lohnhöhe (Leistungsindikator), } \\
\text { konditionelles Lohndifferenzial, } \\
\text { Humankapital, Organisationsgroße }\end{array}$ & $\begin{array}{l}\text { Lohnstreuung bis zu einem gewissen Grad } \\
\text { produktivitatsfordemd }\end{array}$ \\
\hline
\end{tabular}




\begin{tabular}{|c|c|c|c|c|c|c|}
\hline Autor/-in & Jahr & Thema & Daten & Branche & relevante Variable & Ergebnisse \\
\hline $\begin{array}{l}\text { Lallemand. } \\
\text { Plasman, } \\
\text { Rycx }\end{array}$ & 2004 & Produktivităt & Belgien 1995, LEE-Daten & verschiedene & $\begin{array}{l}\text { Gewinn/Mitarbeiter, konditionelle } \\
\text { Lohnstreuung, Standardabweichung Lohn, } \\
\text { Variationskoeffizient, Humankapital, } \\
\text { Org.größe, Branche }\end{array}$ & Lohnstreuung erhőht Produktivităt \\
\hline Jirjahn, Kraft & 2007 & Produktivităt & $\begin{array}{l}\text { Deutschland 1997, } \\
\text { Organisationsdaten }\end{array}$ & $\begin{array}{l}\text { produ-zierendes } \\
\text { Gewerbe }\end{array}$ & $\begin{array}{l}\text { Wertschöpfung pro AN,Org.größe, } \\
\text { Humankapital, Differenz } \\
\text { höchster/niedrigster Lohn }\end{array}$ & $\begin{array}{l}\text { Lohnstreuung erhöht Produktivität } \\
\text { schwach }\end{array}$ \\
\hline $\begin{array}{l}\text { Beaumont, } \\
\text { Harris }\end{array}$ & 2003 & Produktivităt & $\begin{array}{l}\text { GB 1978-95, } \\
\text { Organisationspaneldaten }\end{array}$ & $\begin{array}{l}\text { produ-zierendes } \\
\text { Gewerbe }\end{array}$ & $\begin{array}{l}\text { Produktivităt, Verhältnis Lohn } \\
\text { Arbeiter/Angestellte,Kapitalstock, Anz. } \\
\text { AN, Prozent Angestellte, Alter d. Org., } \\
\text { Branche, Region }\end{array}$ & $\begin{array}{l}\text { Lohnstreuung erhöh//senkt Produktivităt } \\
\text { je nach Branche }\end{array}$ \\
\hline Heymann & 2005 & Produktivităt & $\begin{array}{l}\text { Schweden 1991-95, LEE- } \\
\text { Daten }\end{array}$ & verschiedene & $\begin{array}{l}\text { konditionelles Lohndifferential, } \\
\text { Lohnperzentile, Variationskoeffizient d. } \\
\text { Lohns, Humankapital, Branche }\end{array}$ & $\begin{array}{l}\text { Lohnstreuung erhőht Produktivităt bei } \\
\text { Angestellten }\end{array}$ \\
\hline $\begin{array}{l}\text { Hibbs. } \\
\text { Locking }\end{array}$ & 2000 & Produktivităt & Schweden 1964-93, & $\begin{array}{l}\text { produ-zierendes } \\
\text { Gewerbe }\end{array}$ & $\begin{array}{l}\text { reale Wertschöpfung, } \\
\text { Variationskoeffizient, Arbeitsstunden } \\
\text { durch Arbeiter/-innen, Kapital, } \\
\text { Kapitalkosten }\end{array}$ & Lohnstreuung erhöht Produktivităt \\
\hline
\end{tabular}

\section{Quelle: eigene Darstellung}




\begin{tabular}{|c|c|c|c|c|c|c|c|}
\hline & \multicolumn{7}{|c|}{ NPO / NP-relevante Branchen } \\
\hline \multirow{7}{*}{ 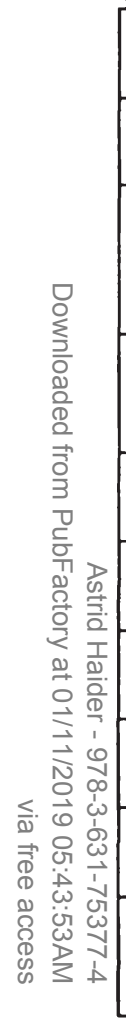 } & 2000 & Lohnstreuung & $\begin{array}{l}\text { USA 1990, } \\
\text { Individualdaten }\end{array}$ & verschiedene & $\begin{array}{l}\text { Varianz det Residuen der } \\
\text { Lohnfunktion }\end{array}$ & $\begin{array}{l}\text { Lohnstreuung im NP-Sektor } \\
\text { geringer }\end{array}$ & $\begin{array}{l}\text { keine Unterscheidung } \\
\text { zwischen innet- } u \text {. } \\
\text { zwischenbetr. Streuung }\end{array}$ \\
\hline & 1993 & Lohnstreuung & $\begin{array}{c}\text { USA } 1969, \\
\text { Individualdaten }\end{array}$ & Universitäten & $\begin{array}{l}\text { Forschungsprodukt ivität, } \\
\text { Lohnstreuung } \\
\text { (Variationskoeffizient), } \\
\text { Arbeitszufriedenheit, } \\
\text { Humankapital }\end{array}$ & $\begin{array}{l}\text { niedrige Lohnstreuung führt zu } \\
\text { höherer Produkt ivität }\end{array}$ & $\begin{array}{c}\text { keine gesonderte } \\
\text { Untersuchung für NP Sektor }\end{array}$ \\
\hline & 2000 & Lohnstreuung & $\begin{array}{l}\text { USA } 1985-98, \\
\text { Organisations- } \\
\text { paneldaten }\end{array}$ & Sport & $\begin{array}{c}\text { gewonnene Spiele, } \\
\begin{array}{c}\text { Personalausgaben, } \\
\text { innerbetriebliche Lohnstreuung } \\
\text { (Herfindahl-Index) }\end{array}\end{array}$ & $\begin{array}{l}\text { höhere Lohngleichheit führt zu } \\
\text { höherer Leistung }\end{array}$ & $\begin{array}{c}\text { keine gesonderte } \\
\text { Untersuchung für NP Sektor }\end{array}$ \\
\hline & 1999 & $\begin{array}{c}\text { Löhne } \\
\text { Führungskräfte }\end{array}$ & $\begin{array}{l}\text { USA 1992, } \\
\text { Organisations- } \\
\text { daten }\end{array}$ & Krankenhaus & $\begin{array}{c}\text { Lohn, Anzahl Betten, Region, } \\
\text { Stelle }\end{array}$ & \begin{tabular}{|c|} 
niedrigere Löhne im NP-Sektor, \\
mehr leistungsabhängige Zahlung \\
in FP-Sektor
\end{tabular} & $\cdots$ \\
\hline & 2002 & $\begin{array}{c}\text { Löhne } \\
\text { Führungskräfte }\end{array}$ & $\begin{array}{l}\text { USA 1992-96, } \\
\text { Organisations- } \\
\text { paneldaten }\end{array}$ & verschiodene & $\begin{array}{l}\text { Lohn, Subventionen, } \\
\text { Nettovermögen }\end{array}$ & $\begin{array}{l}\text { Subventionen haben positiven } \\
\text { Einfluss auf Lohnhöhe }\end{array}$ & Subventionen einbezogen \\
\hline & 2001 & $\begin{array}{c}\text { Löhne } \\
\text { Führungskräfte }\end{array}$ & $\begin{array}{l}\text { USA 1993-95, } \\
\text { Organisations- } \\
\text { paneldaten }\end{array}$ & verschiedene & $\begin{array}{l}\text { Lohn, Einnahmen d. Org, } \\
\text { A usgaben, Anteil Umsatzerlöse }\end{array}$ & \begin{tabular}{|c|}
$\begin{array}{c}\text { Lohn hängt wenig mit Leistung } \\
\text { zusammen, Orggröße positiven } \\
\text { Einfluss }\end{array}$ \\
\end{tabular} & -- \\
\hline & 2003 & $\begin{array}{c}\text { Lōhne } \\
\text { Führungskräfte }\end{array}$ & $\begin{array}{l}\text { USA 2001, } \\
\text { Organisations- } \\
\text { daten }\end{array}$ & $\begin{array}{l}\text { Wirtschafts- } \\
\text { forderung }\end{array}$ & $\begin{array}{c}\text { Lohn, Ausbildung, Geschlecht, } \\
\text { Orggöße. } \\
\text { Organisationsleistung }\end{array}$ & \begin{tabular}{|c|}
$\begin{array}{c}\text { Orggöße hat positiven Einfluss, } \\
\text { Organisationsleistung } \\
\text { keinen/schwach positiven Einfluss } \\
\text { auf Lohnhöhe }\end{array}$ \\
\end{tabular} & -- \\
\hline
\end{tabular}

Quelle: eigene Darstellung 
In diesem Kapitel wird der Modellrahmen zur Berechnung der Einflussfaktoren der innerbetrieblichen Lohnstreuung dargestellt. Außerdem werden verschiedene Streuungsmaße vorgestellt, sowie Hypothesen und erwartete Vorzeichen zum Einfluss einzelner Größen auf die Lohnstreuung diskutiert.

\subsection{Modellrahmen}

Das Modell zur Schätzung der innerbetrieblichen Lohnstreuung kann wie folgt beschrieben werden: ${ }^{59}$

(19) $\operatorname{disp}_{j}=\alpha+X_{j} \beta+Y_{j} \gamma+\varepsilon_{j}$

wobei disp, die innerbetriebliche Lohnstreuung in einer NPO darstellt, $X_{j}$ einen Vektor von Variablen, der die Haupteigenschaften der bezahlten Arbeitnehmer/innen der Organisation $j$ beschreibt. Dazu zählen die Streuung des Alters der Arbeitnehmer/-innen und die Zusammensetzung nach Geschlecht, Erwerbsstatus (d.h. Arbeiter/-innen, Angestellte) und Arbeitszeit (d.h. Vollzeit oder Teilzeit). $Y$, ist ein Vektor von Variablen, der die organisationsspezifischen Komponenten der Organisation $j$ beinhaltet. Dieser beinhaltet zwei Kollektivvertragsdummys, den Aktivitätsbereich der Organisation, eine Variable, die Relation der ehrenamtlichen zu den bezahlten Mitarbeiter/-inne/n beschreibt, Angaben zur Finanzierungsstruktur der Organisation - das sind Einnahmen aus Spenden, Subventionen und aus Leistungsverträgen mit der öffentlichen Hand in Relation zu den Gesamteinnahmen - sowie Angaben zur Organisationsgröße.

Die Messung der Lohnstreuung innerhalb einer Organisation (disp $p_{j}$ ) kann auf unterschiedliche Art erfolgen. Auf die konkrete Ausgestaltung dieser Variable wird deshalb genauer im Kapitel 13.2.1 eingegangen. Wie sich gezeigt hat, hat keine der unterschiedlichen Ausgestaltungen dieser Variable besondere Vorzüge gegenüber den anderen (vgl. Canal Dominguez, Rodriguez Gutierrez 2004: 488). Auch ist anhand der vorhandenen Literatur nicht erkennbar, dass sich ein bestimmtes $\mathrm{Maß}$ in der empirischen Forschung etabliert hat.

Zusätzlich zum Dispersionsmaß auf der linken Seite der Gleichung schlagen manche Autor/-inn/en vor, den Durchschnittslohn in einer Organisation $j$ in die Gleichung als erklärende Variable aufzunehmen, sodass Gleichung (19) in folgender Form geschrieben werden kann (vgl. z.B. Canal Dominguez, Rodriguez Gutierrez 2004):

(20) $\operatorname{disp}_{j}=\alpha+X_{j} \beta+Y_{j} \gamma+\overline{w_{j}} \delta+\varepsilon_{j}$

59 Die Darstellung folgt in Teilen Canal Dominguez und Rodriguez Gutierrez (2004). 
Der durchschnittliche Lohn einer Organisation $\bar{w}$, soll einen möglichen Effekt der Lohnhöhe auf die Lohnstreuung innerhalb einer Organisation berücksichtigen. Kahn und Curme (1987) argumentieren, dass der mittlere Lohn als Proxy für das Humankapital in einer Firma gesehen werden kann (vgl. Kahn, Curme 1987: 603). Dabei kann entweder der Mittelwert der logarithmierten Löhne verwendet werden oder auch der Logarithmus der Mittelwerte der Löhne der Organisationen, je nach verwendetem Dispersionsmaß. Das Einfügen der mittleren Lohnhöhe einer Organisation kann statistisch erklärt werden, da so eine Anlehnung an den Variationskoeffizienten erfolgt, was in Kapitel 13.2.1 noch ausführlich erläutert wird.

Die Einführung des Durchschnittslohns $\bar{w}_{j}$ kann jedoch ein Endogenitätsproblem hervorrufen, da unklar ist, ob die Lohnhöhe die Lohnstreuung beeinflusst oder umgekehrt. Deshalb wird diese Variable instrumentiert. Als Instrumentvariable (IV) wird der aus Gleichung (10) geschätzte durchschnittliche



$$
\text { disp }=\alpha+X, \beta+Y_{,} \gamma+\overline{\hat{w}}_{\jmath} \delta^{\prime \prime}+\varepsilon
$$

Damit wird ein zweistufiges Schätzverfahren (2SLS) für die Schätzung der innerbetrieblichen Lohnstreuung notwendig. ${ }^{60}$

\subsection{Spezifikation der Variablen}

In diesem Kapitel werden die verwendeten Variablen spezifiziert. Im ersten Unterkapitel wird das Streuungsmaß, das als abhängige Variable auf der linken Seite der Gleichung (21) steht, spezifiziert, danach werden die erklärenden Variablen näher dargestellt.

\subsubsection{Berechnung der Lohnstreuung in einer Organisation}

Zur Messung von Einkommensungleichheit bzw. Einkommensverteilung wurde eine Reihe von Maßzahlen entwickelt, von denen die verwendeten in Folge kurz vorgestellt werden sollen, wobei von einer ausführlichen Diskussion der Vorund Nachteile der einzelnen Maßzahlen an dieser Stelle abgesehen wird. ${ }^{61}$ In der vorhandenen empirischen Literatur, in der die innerbetriebliche Lohnstreuung untersucht wird, werden verschiedene Maße verwendet. Keines der Maße weist einen besonderen Vorzug gegenüber den anderen auf, sodass in der vorliegenden Arbeit unterschiedliche Kennzahlen verwendet werden. Es werden jene Maße dargestellt, die auch in empirischen Arbeiten, die sich mit Determinanten

60 Für eine ausführlichere Darstellung des zweistufigen Schätzverfahrens siehe Kapitel 14.3.




der Lohnstreuung beschäftigen, zu finden sind. Ziel ist es zu unter-suchen, ob die verschiedenen $\mathrm{Maße} z u$ anderen Ergebnissen führen.

Prinzipiell kann die Messung entweder durch den Vergleich von nur zwei Löhnen - etwa der Messung der Differenz zwischen dem Lohn der leitenden Person und dem eines bzw. einer durchschnittlichen Angestellten - in einer Firma gemacht werden oder durch die Messung der Verteilung der Löhne in einer Organisation. Für letzteren Zugang können beispielsweise die Standardabweichung, die Varianz der Löhne oder der Gini-Koeffizient zur Darstellung der Verteilung der Ein-kommen innerhalb einer Organisation berechnet werden. Die erste Möglichkeit ist einfacher zu berechnen, da hierfür weniger Daten hierfür benötigt werden, hat aber gleichzeitig den Nachteil, dass der Quotient von nur zwei Löhnen, etwa des Lohns der leitenden Person und eines bzw. einer durchschnittlichen Angestellten, sehr sensibel reagiert. Der Grund hierfür ist, dass mitunter der Lohn der leitenden Person extreme Werte annehmen kann, was die tatsächliche Verteilung innerhalb einer Organisation nicht widerspiegelt (vgl. Beaumont, Harris 2003: 55). Außerdem wird dabei das untere Lohnsegment vollkommen ignoriert.

Ein einfaches Maß zu Messung von Lohnungleichheit, das zu der ersten oben genannten Gruppe zählt, ist der Abstand $R_{j}$ (englisch: range), der folgendermaBen berechnet wird:

$$
R_{J}=w_{\text {max }}-w_{\text {min }}
$$

wobei $w_{\max }$ für den höchsten Lohn der Organisation steht und $w_{\min }$ für den niedrigsten. Hier wird schnell klar, dass ein kleiner Wert $R_{j}$ eine gleichere Lohnverteilung darstellt, ein hoher eine ungleichere Verteilung. Ebenso wird schnell klar, dass der Wert $R$, sehr von den beiden konkreten Zahlen abhängt. Ausreißer nach oben und nach unten verändern das Maß. Sehr viel Information, die über die tatsächliche Lohnverteilung vorliegt, wird für die Berechnung dieses Maßes nicht verwendet. Ein Vorteil dieses Maßes ist, dass keine Lohninformationen zu allen Arbeitnehmer/-innen vorliegen müssen. Außerdem ist eventuell besonders der höchste Lohn innerhalb einer Organisation für viele Arbeitnehmer/-innen als Vergleichsmaßstab relevant. Obwohl die Löhne der Höchstverdiener/-innen als „Ausreißer“ im Vergleich zu den restlichen Löhnen in einer Firma betrachtet werden können, sind sie in dieser Arbeit zum Teil von Interesse.

Eine weitere Möglichkeit sind Rangfolgen, wozu Quantile benutzt werden. Auch hier werden nur jeweils zwei Löhne gegenübergestellt. Üblich sind folgende Maßzahlen:

$$
\frac{Q_{0,9}}{Q_{0,5}}, \frac{Q_{0,75}}{Q_{0,25}}, \frac{Q_{0,5}}{Q_{0,1}}, \frac{Q_{0,9}}{Q_{0,1}}
$$

wobei beispielsweise $Q_{0.9}$ das 0,9 Lohnquantil in einer Organisation darstellt, d.h. 90\% aller Löhne in der Organisation liegen unter, 10\% über diesem Lohn. 
$\mathrm{Zu}$ den Lohnstreuungsmaßen, die die Verteilung der Löhne analysieren, zählen die Varianz $V$, bzw. die Standardabweichung $S$, Sie werden berechnet als

$$
\begin{aligned}
& V_{j}=\frac{1}{n} \sum_{i=1}^{n}\left[w_{i j}-\bar{w}_{J}\right]^{2} \\
& S_{J}=\sqrt{\frac{1}{n} \sum_{i=1}^{n}\left[w_{l j}-\bar{w}_{J}\right]^{2}}
\end{aligned}
$$

wobei $w_{y}$ der Lohn eines Arbeitnehmers bzw. einer Arbeitnehmerin in der Organisation $j$ ist, $\bar{w}$, der mittlere Lohn der Organisation $j . n$ stellt die Anzahl der Arbeitnehmer/-innen in der Organisation dar. Durch das Quadrieren der Abweichung werden größere Abweichungen stärker gewichtet. Die Standardabweichung und die Varianz sind übliche Streuungsmaße in der Statistik, haben aber den Nachteil, dass sich bei Verdopplung aller Löhne, die Standardabweichung ebenfalls verdoppelt, die Varianz sogar vervierfacht wird, obwohl sich an der Verteilung an sich nichts geändert hat (vgl. Blümle 1975: 38, Cowell 1995: 24). Aus diesem Grund werden häufig zwei weitere Maße herangezogen, nämlich die Standardabweichung der logarithmierten Einkommen und der Logarithmus der Standardabweichungen. Sie werden berechnet als

$$
\begin{gathered}
v_{1}=\frac{1}{n} \sum_{i=1}^{n}\left[\log \left(\frac{w_{i j}}{\bar{w}_{j}}\right)\right]^{2} \text { und } \\
\text { (25) } v_{2}=\frac{1}{n} \sum_{i=1}^{n}\left[\log \left(\frac{w_{i j}}{w_{j}}\right)\right]^{2} \text { wobei } \\
w_{j}^{\cdot}=\exp \left(\frac{1}{n} \sum_{i=1}^{n} \log w_{l j}\right) .
\end{gathered}
$$

$w^{*}$ stellt das geometrische Mittel dar. Während die erste Gleichung als logarithmische Varianz bezeichnet wird, ist die zweite die Varianz der Logarithmen der Löhne. Während $v_{1}$ relativ zum Logarithmus des mittleren Lohns definiert ist, definiert sich $v_{2}$ relativ zum Mittelwert des Logarithmus des Einkommens (vgl. Cowell 1995: 25).

Zueinander verhalten sich die beiden Maße folgendermaßen (vgl. Cowell 1995: 149):

(26) $v_{1}=v_{2}+\left[\log \left(\frac{w_{j}}{\bar{w}_{j}}\right)\right]^{2}$

Die Standardabweichung der logarithmierten Löhne ist ein in der empirischen Literatur relativ häufig verwendetes $\mathrm{Ma} ß$ zur Streuungsmessung. Als Vorteil führen Dell'Aringa und Lucifora (1994: 156) an, dass relative und nicht absolute 
Vergleiche gemacht werden. Außerdem bezeichnen sie es am geeignetsten, wenn die Löhne durch eine semi-logarithmische Lohnfunktion bestimmt werden und wenn Löhne approximativ logarithmisch normal verteilt sind (vgl. auch Freeman 1980: 6; Freeman 1982: 6). Die Standardabweichung der logarithmierten Löhne gibt tendenziell Ungleichheit am unteren Ende der Verteilung mehr Gewicht (vgl. Freeman 1980: 6).

Eine weitere Möglichkeit, das Problem zu vermeiden, dass eine Vervielfachung der Löhne zu einer Vervielfachung des Verteilungsmaßes führt, ist der Variationskoeffizient $c_{j}{ }^{62}$, bei dem die Standardabweichung der (logarithmierten) Löhne in einer Organisation durch den mittleren Lohn in der Organisation dividiert wird:

(27) $c_{j}=\frac{\sqrt{V_{j}}}{\bar{w}_{j}}$

Dieses $\mathrm{Ma}$ ist unempfindlich gegenüber proportionalen Transformationen. Die Standardabweichung, Varianz und der Variationskoeffizient steigen mit zunehmender Ungleichheit der Löhne.

Natürlich gibt es auch andere Maße zur Messung der Lohnstreuung, wie z.B. den Gini-Koeffizienten oder das Atkinson Maß (vgl. Cowell 1995). In der empirischen Literatur, die Determinanten der Lohnstreuung untersucht, werden diese Maße meines Wissens nach jedoch nicht oder nur selten verwendet.

Wie eingangs festgestellt, hat sich keines der Maße in der empirischen vorhandenen Literatur als gängig durchgesetzt. Zum Teil werden auch mehrere Maße nebeneinander verwendet, unter anderem um die Robustheit der Ergebnisse zu überprüfen. Zur Messung der Lohnspreizung verwenden Freeman (1980), ebenso wie Dell'Aringa und Lucifora (1994) die Standardabweichung der logarithmierten Löhne. Freeman (1982: 9) und James und Morlock (1987: 971) verwenden, die Varianz der logarithmierten Löhne bei der Analyse der Einflussfaktoren der Lohnstreuung und präsentieren als Alternativmaß die Standardabweichung der logarithmierten Löhne im Anhang. Auch Bechtel, Heinbach, Strotmann (2005) verwenden die Standard-abweichung der logarithmierten Löhne. Pfeffer und Davis-Blake (1990: 44) verwenden den Variationskoeffizienten, führen jedoch nicht näher aus, ob - wie in der Lohnliteratur üblich logarithmierte Werte verwendet werden.

Canal Dominguez und Rodriguez Gutierrez (2004: 488) verwenden die logarithmierten Standardabweichungen der Löhne in einem Unter-nehmen als Dispersionsmaß, fügen aber zusätzlich als unabhängige Variable den mittleren Lohn pro Firma in die Gleichung ein. Der Grund dafür liegt darin, dass sie den Varia- 
tionskoeffizienten als das statistisch geeignetste Maß erachten. Dadurch ist die Streuung nicht vom Lohnniveau einer Firma abhängig. Logarithmiert man nun jedoch den Variationskoeffizienten, wie in der empirischen Literatur für Löhne üblich, so folgt

$\log \left(\frac{\sqrt{V_{j}}}{\bar{w}_{j}}\right)=\log \sqrt{V_{j}}-\log \bar{w}_{j}$.

Wenn nun der zweite Term $\log \bar{w}_{j}$ auf die rechte Seite der Modellgleichung (20) gebracht wird, müsste der Koeffizient $\delta$ ex ante auf 1 gesetzt werden, um ein dem Variationskoeffizienten äquivalentes Ergebnis zu erhalten. Diese Einschränkung wird jedoch von den Autoren nicht von vornherein gemacht (vgl. Canal Dominguez, Rodriguez Gutierrez 2004: 488).

Auch in dieser Arbeit wird so vorgegangen, dass der Durchschnittslohn als unabhängige Variable zusätzlich eingefügt wird, der Koeffizient $\delta$ dieser Variable wird nicht ex ante auf 1 gesetzt. Das Maß entspricht daher nicht genau dem Variationskoeffizienten, ist jedoch eine Annäherung an diesen.

Interessanterweise thematisieren die Autor/-inn/en der verschiedenen Studien nicht oder nur in begrenztem Ausmaß, warum sie sich für das jeweilige Dispersionsmaß entschieden haben. Auch diskutieren sie wenig bis gar nicht, inwiefern sich Ergebnisse bei Verwendung eines anderen Dispersionsmaßes verändern würden. Es ist nicht a priori klar, dass unter Verwendung unterschiedlicher Streuungsmaße dieselben Ergebnisse erzielt werden. Eine Diskussion über mögliche Ver-änderungen der Ergebnisse wäre deshalb wichtig und wünschenswert. Zum Teil werden die Leser/-innen auch im Unklaren darüber gelassen, welches $\mathrm{Ma}$ ß genau verwendet wurde. So beschreiben Canal Dominguez und Rodriguez Gutierrez (2004: 488f.) zum Beispiel, dass sie den Logarithmus der Standardabweichungen der Löhne als abhängige Variable und den Logarithmus der durchschnittlichen Löhne als unabhängige Variable verwenden, führen folgend jedoch aus, dass sie den durchschnittlichen Lohn als Durchschnitt eines geschätzten logarithmierten Lohns instrumentieren. So bleibt unklar, ob sie den Durchschnitt des logarithmierten Lohns oder den Logarithmus des durchschnittlichen Lohns verwenden, was nicht dasselbe ist.

All die oben genannten Indikatoren sind unkonditionelle Maße, d.h. die Lohnstreuung wird in einem Unternehmen gemessen, ohne dabei die Zusammensetzung der Arbeitnehmer/-innen in der Organisation zu beachten. Das Gegenteil wäre ein konditionelles $\mathrm{Ma} ß$, das auch als residuale Lohnstreuung bezeichnet wird. Lohnungleichheit wird dabei gemessen, nachdem für Unterschiede im Humankapital kontrolliert wurde. Aus dem Lohnresidual wird ein Ungleichheitsmaß gebildet, etwa die Standardabweichung (vgl. z.B. Lallemand et al. 2004: 542). Es macht vor allem dann Sinn, ein konditionelles Maß zu verwenden, wenn Lohnunterschiede von vermeintlich gleichen Arbeitnehmer/-innen 
untersucht werden sollen. ${ }^{63}$ Konditionelle Lohnungleichheitsmaße findet man vor allem in der Literatur, die sich mit dem Zusammenhang zwischen Lohnungleichheit und Produktivität beschäftigt. Sowohl Winter-Ebmer und Zweimüller (1999) als auch Lallemand, Plasman und Rycx (2004 und 2007b) sowie Heymann (2005) verwenden die Standardfehler, die durch Regressionen von Einflussfaktoren auf den Lohn auf Individualebene berechnet werden, als konditionelles Ungleichheitsmaß. Dabei wird die Gleichung für jede Firma getrennt berechnet (vgl. z.B. Lallemand et al. 2004: 542). Ziel ist es, Einflussfaktoren von Lohnungleichheit, die nicht auf offensichtlichen Unterschieden im Humankapital beruhen, auf die Produktivität zu untersuchen. Heymann (2005: 1319) verwendet alternativ auch den Variationskoeffizienten und den Quotienten des 0,9 und 0,1 Perzentil als unkonditionelle Maße zur Überprüfung der Robustheit der Ergebnisse. Jirjahn und Kraft (2007) verwenden den Abstand $R_{j}$ zwischen dem höchsten und dem niedrigsten Lohn als unkonditionelles Maß.

Die Schätzungen im anschließenden Kapitel erfolgen mit mehreren Ungleichheitsmaßen als abhängiger Variable. Einerseits soll damit die Robustheit der Ergebnisse überprüft werden, andererseits soll damit auch die Unsicherheit in der vorhandenen empirischen Literatur über die Verwendung dieser Maße widergespiegelt werden. Verwendet werden die Standardabweichung der logarithmierten Löhne, der $Q 0,9 / Q 0,1$-Koeffizient, die Varianz der logarithmierten Löhne und die Logarithmen der Standardabweichungen der Löhne als abhängige Variable, da sie auch in anderen Arbeiten die Maße sind, die am häufigsten verwendet wurden. Für die vorliegende Untersuchung sind unkonditionelle Streuungsmaße sinnvoller, da die Lohnungleichheit innerhalb einer NPO gemessen werden soll. Dabei ist davon auszugehen, dass innerhalb einer Organisation Personen mit unterschiedlichem Humankapital beschäftigt sind. Es sollen Lohnungleichheiten unter ungleichen Individuen gemessen werden.

\subsubsection{Spezifikation der erklärenden Variablen}

Als Variablen, die die Zusammensetzung der bezahlten Belegschaft innerhalb der Organisation beschreiben, gehen die Streuung des Alters, die Verteilung von Männern und Frauen, von Arbeiter/-inne/n und Angestellten sowie von Vollzeitund Teilzeitkräfte in einer Organisation ein. Die Variablen dienen dazu, für Unterschiede im Humankapital zu kontrollieren. Wie auch im ersten Teil der Dissertation wäre es wichtig und wünschenswert, genauere Angaben zu den Individuen und deren Unterschieden im Humankapital, in der Tätigkeit und verschiedenen Verantwortungsbereichen als erklärende Variablen einzubauen. Leider ist dies aufgrund mangelnder Datenverfügbarkeit nicht möglich.

63 So würden sich konditionelle Lohnungleichheitsmaße für die Untersuchung von Diskriminierung in einer Organisation eignen. 
Im Gegensatz zum ersten Teil der Dissertation gehen jedoch nicht Daten auf Individualebene in die Gleichung ein, sondern aggregierte Individualdaten auf Organisationsebene. Es werden daher jeweils die Standardabweichung bzw. die Varianz je Organisation aus den einzelnen Individualdaten errechnet.

In verschiedenen empirischen Studien werden auch Anteile in Prozent oder Durchschnittswerte verwendet, um die Zusammensetzung der Belegschaft zu beschreiben (vgl. z.B. Bechtel et al. 2005, Canal Dominguez, Rodriguez Gutierrez 2004, Pfeffer, Davis-Blake 1990). Im Vergleich zu Prozentwerten sind meines Erachtens Streuungsmaße wie die Varianz oder die Standardabweichung jedoch vorzuziehen. Der Grund ist ein vermuteter nicht-linearer Verlauf des $\mathrm{Zu}$ sammenhangs zwischen den unabhängigen Variablen und der Lohnstreuung. Die Lohnstreuung ist nämlich dann am größten, wenn die Arbeitnehmer/-innen einer Organisation am heterogensten sind. Arbeiten z.B. zu 50\% Männer und zu $50 \%$ Frauen in einer Organisation, so ist bei vermuteter unterschiedlicher $\mathrm{Be}$ zahlung der beiden Gruppen die Lohnstreuung im Vergleich zu einer Organisation, wo nur Männer oder nur Frauen arbeiten, größer. Würden Prozentwerte verwendet, so würde bei einem linearen Verlauf unterstellt werden, dass beispielsweise die Lohn-streuung in einer Organisation, in der nur Männer arbeiten niedriger ist als in einer, in der ausschließlich Frauen arbeiten. Dies erscheint jedoch nicht plausibel, vielmehr würden sich die beiden Organisationen durch den mittleren Lohn unterscheiden, nicht jedoch durch die Lohnstreuung. ${ }^{64}$ Der Zusammenhang zwischen dem Frauenanteil in einer Organisation und der Lohnstreuung kann somit als umgekehrt U-förmig beschrieben werden. Das Beispiel sei noch einmal durch folgendes Zahlenbeispiel verdeutlicht:

Wir haben im folgenden Beispieldatensatz sieben Organisationen, Organisation 1 beschäftigt ausschließlich Männer (Wert 0), Organisation 7 ausschließlich Frauen (Wert 1). Während die Standardabweichung und die Varianz des Geschlechts ihr Maximum bei Organisation 4 erreichen, der Organisation, die Männer und Frauen zu ausgeglichenen Teilen beschäftigt, steigen die Prozentwerte stetig und erreichen ihren höchsten Wert bei Organisation 7.

64 Dies setzt wiederum voraus, dass es in diesem Fall keinen Zusammenhang zwischen der mittleren Lohnhöhe und der Lohnstreuung gibt. 
Tabelle 25: Beispieldatensatz

\begin{tabular}{|c|c|c|c|c|c|c|}
\hline Org. 1 & Org. 2 & Org. 3 & Org. 4 & Org. 5 & Org. 6 & Org. 7 \\
\hline 0 & 1 & 1 & 1 & 1 & 1 & 1 \\
\hline 0 & 0 & 1 & 1 & 1 & 1 & 1 \\
\hline 0 & 0 & 0 & 1 & 1 & 1 & 1 \\
\hline 0 & 0 & 0 & 0 & 1 & 1 & 1 \\
\hline 0 & 0 & 0 & 0 & 0 & 1 & 1 \\
\hline 0 & 0 & 0 & 0 & 0 & 0 & 1 \\
\hline \multicolumn{7}{|c|}{ Standardabweichung des Geschlechts } \\
\hline 0,0000 & 0,4082 & 0,5164 & 0,5477 & 0,5164 & 0,4082 & 0,0000 \\
\hline \multicolumn{7}{|c|}{ Varianz des Geschlechts } \\
\hline 0,0000 & 0,1667 & 0,2667 & 0,3000 & 0,2667 & 0,1667 & 0,0000 \\
\hline \multicolumn{7}{|c|}{ Anteil Frauen } \\
\hline 0,0000 & 0,1667 & 0,3333 & 0,5000 & 0,6667 & 0,8333 & 1,0000 \\
\hline
\end{tabular}

Zusätzlich wird Männern und Frauen ein fiktiver Lohn zugeordnet. Im Beispiel verdienen Männer 10 Geldeinheiten, Frauen 8. Daraus ergibt sich in diesen Organisationen folgende Standardabweichung und Varianz der Löhne:

Tabelle 26: Standardabweichung und Varianz im Beispieldatensatz
\begin{tabular}{l|l|l|l|l|l|l|} 
Org. 1 & Org. 2 & Org. 3 | Org. 4 & Org.5 & Org.6 & Org.7
\end{tabular} Standardabweichung der Löhne
$0,0000|0,8165| 1,0328|1,0954| 1,0328|0,8165| 0,0000$
Varianz der Löhne
\begin{tabular}{l|l|l|l|l|l|l}
0,0000 & 0,6667 & 1,0667 & 1,2000 & 1,0667 & 0,6667 & 0,0000
\end{tabular}

Die folgende Abbildung 2 stellt anhand des numerischen Beispiels die Korrelation zwischen den Variablen „Anteil Frauen“, der Standardabweichung und der Varianz des Geschlechts in der Organisation und der Standardabweichung und der Varianz der Löhne dar. Hier wird klar ersichtlich, dass der Zusammenhang zwischen dem Anteil der weiblichen Mitarbeiter und der Standardabweichung (bzw. Varianz) der Löhne als umgekehrt U-förmig beschrieben werden kann, jener zwischen der Standardabweichung (bzw. der Varianz) des Geschlechts und der Standardabweichung (bzw. Varianz) der Löhne als linear.

Gleich verhält es sich bei den Variablen Streuung des Alters, der Arbeiter / Angestellten sowie der Voll- und Teilzeitkräfte. 


\section{Abbildung 2: Korrelation Geschlecht und Lohn}

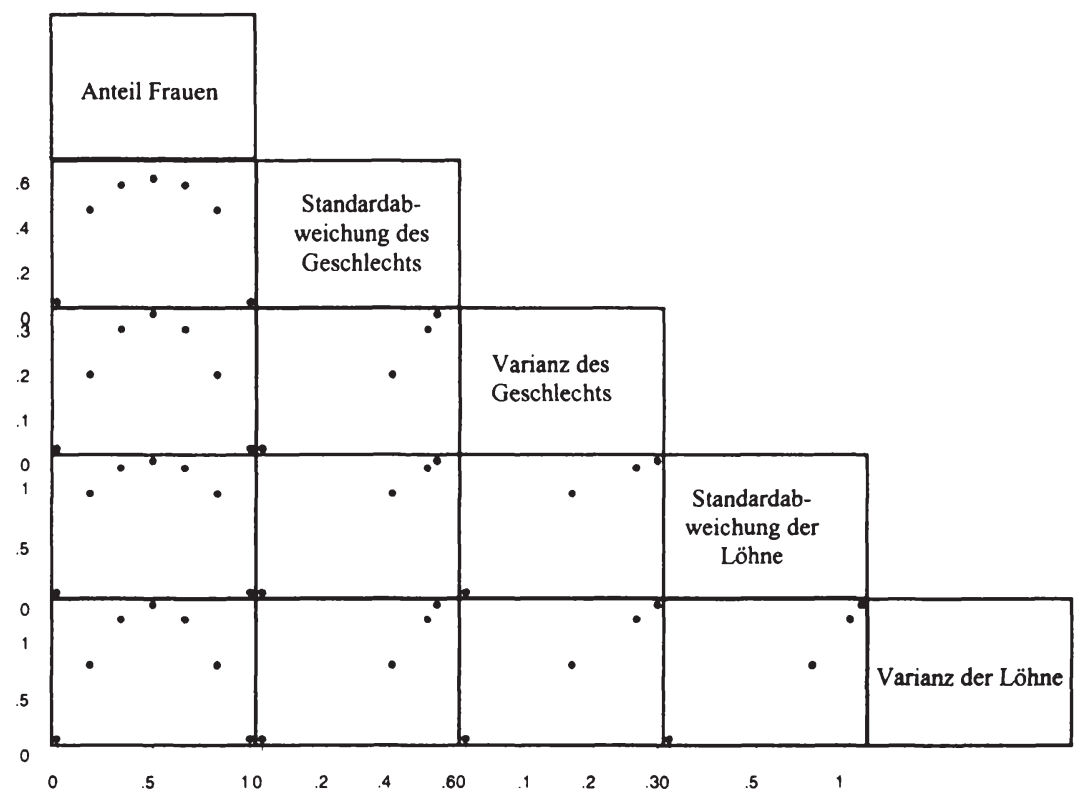

Quelle: eigene Darstellung

Auch die Streuung der ehrenamtlichen Mitarbeiter/-innen in bezug auf die Anzahl der Vollzeitäquivalente folgt derselben Logik wie die Streuung des Alters oder des Geschlechts. Bei Verwendung dieses Maßes geht man davon aus, dass die Lohnstreuung am kleinsten ist, wenn die Zusammensetzung der bezahlten und unbezahlten Arbeitskräfte am heterogensten ist. Würde statt dessen die Anzahl der ehrenamtlichen Mitarbeiter/-innen pro bezahltem Vollzeitäquivalent verwendet werden, wäre die Hypothese, dass die Lohnstreuung mit zunehmender Anzahl unbezahlter Arbeitskräfte sinkt. In dieser Schätzung wird aber der ersten Hypothese der Vorzug gegeben, d.h. als Maß zur Beschreibung des Verhältnisses zwischen freiwilligen und bezahlten Arbeitskräften wird die Streuung der ehrenamtlichen Mitarbeiter/-innen zu den Vollzeit-äquivalenten verwendet, und es wird davon ausgegangen, dass die Lohnstreuung am kleinsten ist, wenn die Zusammensetzung am heterogensten ist.

Zum anderen werden Angaben zur Finanzierungsstruktur der Organisation verwendet. Dazu wird der Anteil der Spenden und der Einkünfte aus Sponsoring an den Gesamteinnahmen, jener von Subventionen bzw. jener der Einnahmen aus Leistungsverträgen mit der öffentlichen Hand an den Gesamteinnahmen verwendet. 
Der Tätigkeitsbereich der NPO wird wiederum mit ÖNACE-Dummys spezifiziert. Da die vorliegende Fallzahl jedoch kleiner ist als im ersten Teil der Arbeit, werden einzelne ÖNACE Gruppen auf 2-Steller-Ebene (vgl. Statistik Austria o.J.) zusammengefasst, um eine ausreichende Fallzahl pro Kategorie zu gewährleisten. Referenzgruppe ist die ÖNACE Kategorie 85, die das Gesundheits-, Veterinär- und Sozialwesen umfasst. Als Referenzgruppe wird sie deshalb herangezogen, weil sie einen der wichtigsten Tätigkeitsbereiche für NPO, die auch bezahlte Mitarbeiter/-innen beschäftigen, umfasst.

Des weiteren werden zwei Kollektivvertragsdummys in die Gleichung eingefügt. Sie geben an, ob eine Organisation keinem Kollektivvertrag unterliegt, sich jedoch in ihrem Lohnschema an einem orientiert oder ob sie keinem unterliegt und sich auch an keinem orientiert. Als Referenzkategorie dienen jene Organisationen, die einem oder mehreren Kollektivverträgen unterliegen. Mit den Dummys soll untersucht werden, ob die Zugehörigkeit zu dem Kollektivvertrag Einfluss auf die Lohnstreuung hat, wie in anderen empirischen Untersuchungen öfters gezeigt wurde (vgl. Kapitel 12.1.2).

Außerdem geht die Anzahl der bezahlten Arbeitnehmer/-innen in Form von Vollzeitäquivalenten in die Schätzgleichung ein. Diese Variable wird in ihrer logarithmierten Form verwendet und stellt einen Maßstab für die Firmengröße dar.

Die mittlere Lohnhöhe geht entweder als Logarithmus des Mittelwerts der Lohnhöhe einer Organisation ein oder als Mittelwert der Logarithmen der Lohnhöhe der Organisation, je nach verwendeter abhängiger Variable.

Im Gegensatz zum ersten Teil der Dissertation gehen Bundesländerdummys nicht in die Schätzgleichung ein. Der Grund dafür liegt darin, dass sowohl theoretisch als auch empirisch keine Argumente dafür gefunden werden können, warum die Lohnstreuung in den verschiedenen Regionen Österreichs unterschiedlich sein sollen.

\subsection{Erwartete Vorzeichen}

In diesem Modell wird folgender Zusammenhang zwischen der innerbetrieblichen Lohnstreuung und den unabhängigen Variablen vermutet: 
erwartetes

\begin{tabular}{|c|c|c|}
\hline & & \\
\hline Standardabw. (Varianz) Alter & Standardabweichung (Varianz) Alter / NPO & + \\
\hline Standardabw. (Varianz) Geschlecht & Standardabw. (Varianz) des Geschlechts / NPO & + \\
\hline Standardabw. (Var.) Beschăftigungsausmaß & Standardabw. (Varianz) der Vollzeit-/Teilzeitkräfte / NPO & + \\
\hline Standardabw. (Varianz) soziale Stellung & Standardabw. (Varianz) der Arbeiter/-innen / Angestellten / NPO & + \\
\hline \multicolumn{3}{|c|}{ freiwillige Zuwendungen } \\
\hline Standardabw. (Varianz) ehrenamtliche Besch. & Standardabw. (Varianz) der ehrenamtl. Mitarbeiter / Vollzeităquivalente & - \\
\hline Anteil Spenden u.Sponsoring/Gesamteinnahmen & Anteil der Einnahmen aus Spenden und Sponsoring an den Gesamteinnahmen & $+1-$ \\
\hline Anteil Subvention/Gesamteinnahmen & Anteil der Subventionen an den Gesamteinnahmen & $+1-$ \\
\hline \multicolumn{3}{|c|}{ andere Organisationsvariablen } \\
\hline Anteil Leistungsvertrag/Gesamteinnahmen & Anteil der Einnahmen aus Leistungsverträgen an den Gesamteinnahmen & $+1-$ \\
\hline ÖNACE 55 & $\begin{array}{l}\text { Dummy Variable, } 1 \text { für Beherbergungs- u. Gaststättenwesen, sonst } 0 \text {, } \\
\text { Referenzgruppe: Gesundheits-, Veterinär- und Sozialwesen }\end{array}$ & I \\
\hline ÖNACE 73 & $\begin{array}{l}\text { Dummy Variable, } 1 \text { für Forschung u. Entwicklung, sonst } 0 \text {, Referenzgruppe: } \\
\text { Gesundheits-, Veterinär- und Sozialwesen }\end{array}$ & I \\
\hline ÖNACE 80 & $\begin{array}{l}\text { Dummy Variable, } 1 \text { für Unterrichtswesen, sonst 0, Referenzgruppe: } \\
\text { Gesundheits-, Veterinär- und Sozialwesen } \\
\text { Dummy Variable, } 1 \text { für Erbringung v. sonstigen öffentl. u. persönl. }\end{array}$ & I \\
\hline ÖNACE 91 & $\begin{array}{l}\text { Dienstleistungen, sonst } 0 \text {, Referenzgruppe: Gesundheits-, Veterinär- und } \\
\text { Sozialwesen }\end{array}$ & I \\
\hline ÖNACE 92 & $\begin{array}{l}\text { Dummy Variable, } 1 \text { für Kultur, Sport u. Unterhaltung, sonst } 0 \text {, } \\
\text { Referenzgruppe: Gesundheits-, Veterinär- und Sozialwesen }\end{array}$ & I \\
\hline durchschnittl. Lohnhöhe & $\begin{array}{l}\text { (geschătzte) durchschnittl. log. Lohnhöhe der Org. bzw. Log. durchschnittl. } \\
\text { Lohnhöhe }\end{array}$ & + \\
\hline kein Kollektivvertrag, aber Orientierung & $\begin{array}{l}\text { Dummy Variable, } 1 \text { wenn Organisation keinem Kollektivvertrag unterliegt, sich } \\
\text { jedoch daran orientiert, sonst } 0 \text {, Referenzgruppe: Organisation hat } \mathbf{K V}\end{array}$ & + \\
\hline $\begin{array}{l}\text { kein Kollektivvertrag, keine Orientierung } \\
\qquad \log . V Z A ̄\end{array}$ & $\begin{array}{l}\text { Dummy Variable, } 1 \text { wenn Organisation keinem Kollektivvertrag unterliegt, } \\
\text { sonst 0, Referenzgruppe: Organisation hat KV } \\
\text { log. Vollzeităguivalente in einer Organisation }\end{array}$ & $\begin{array}{l}+ \\
+\end{array}$ \\
\hline
\end{tabular}


Wie im vorherigen Unterkapitel ausführlich dargestellt, sind die erwarteten Vorzeichen für die Variablen Standardabweichung (Varianz) Alter, weibliche Arbeitnehmer und Vollzeitkräfte positiv. Die Lohnstreuung steigt mit zunehmender Standardabweichung (Varianz). Je nach verwendeter abhängiger Variable, werden mitunter auch die Varianzen dieser Variablen verwendet. Wenn nicht die Standard-abweichung des Lohns als endogene Variable verwendet wird, werden analog dazu die Varianzen der exogenen individualbezogenen Variablen berechnet.

Es wird erwartet, dass ein ausgeglichenes zahlenmäßiges Verhältnis zwischen ehrenamtlichen und bezahlten Mitarbeiter/-innen die Lohnstreuung in NPO schmälert. Ehrenamtliche Arbeit wirkt sich womöglich auf die Hierarchie in einer NPO aus. Beruht nämlich ein Teil der Arbeit in einer Organisation auf ehrenamtlicher Arbeit, so werden einige Stellen in der Organisation durch ebendiese freiwilligen Mitarbeiter/-innen abgedeckt und werden daher nicht bezahlt. Damit fallen einzelne ,Lohnsegmente' innerhalb einer Organisation weg und schmälern die Lohnspreizung. Ehrenamtliche können auch auf einen hohen Stellenwert der Mission der NPO deuten, was zu einem Lohnverzicht insbesondere bei Führungskräften deuten kann und zu einer geringeren Lohnstreuung führen kann.

Der Effekt einer höheren Abhängigkeit von Spenden und Subventionen bleibt unklar, da sowohl Argumente für einen positiven als auch einen negativen Einfluss sprechen. Hohe Lohngruppen profitieren mehr von hohen Spenden und Subventionen, was die Lohnstreuung vergrößern kann. Außerdem wäre es möglich, dass im Fall der Subventionen durch eine Einflussnahme von außen, ein Lohnschema vorgegeben ist, das die Lohnstreuung womöglich vergrößert oder vermindert. NPO hängen jedoch besonders von der öffentlichen Wahrnehmung $a b$, durch eine hohe Lohnspreizung könnte die Legitimität nach außen gefährdet sein. Deshalb könnte eine hohe Abhängigkeit von Spenden und Subventionen auf eine geringe Lohnstreuung deuten, insbesondere im Fall der Spenden.

Unklar bleibt auch, die sich der Anteil der Einnahmen aus Leistungsverträgen an den Gesamteinnahmen auf die Lohnstreuung einer NPO auswirkt. Ähnlich wie bei Subventionen könnten Geldgeber/-innen Einfluss auf die Lohnstreuung nehmen.

Über die erwarteten Vorzeichen der Koeffizienten der einzelnen Branchendummys kann keine genaue Vermutung angestellt werden. Wenn die NPO mehrheitlich professionell agiert, d.h. die meisten ihrer Tätigkeiten durch bezahlte Arbeitskräfte verrichtet, so ist vermutlich die Lohnstreuung größer als wenn eine Organisation nur wenige Stellen durch bezahlte Individuen besetzt. Variieren könnte die Lohnstreuung zwischen Branchen auch aufgrund unterschiedlicher Qualifikations-struktur der bezahlten Beschäftigten zwischen den verschiedenen Branchen. Leider ist eine genauere Messung von Unterschieden 
in der Qualifikationsstruktur im Datensatz aufgrund nicht vorhandener Informationen nicht möglich.

Das erwartete Vorzeichen des Koeffizienten der mittleren Lohnhöhe der Organisationen ist positiv, d.h. je höher der durchschnittliche Organisationslohn ist, desto größer ist die Lohnstreuung. In empirischen Analysen kann in vielen Ländern ein positiver Zusammenhang zwischen der Lohnhöhe und der innerbetrieblichen Lohnstreuung festgestellt werden, d.h. in Firmen mit hohen durchschnittlichen Löhnen ist auch die Lohnstreuung höher. Je nach Land beträgt die Korrelation zwischen 0,1 und 0,3 (vgl. Lazear, Shaw 2007: 15). Gründe für diesen Zusammenhang können einerseits in der Humankapitaltheorie gefunden werden. Kahn und Curme (1987) argumentieren, dass der mittlere Lohn als Proxy für das Humankapital in einer Firma gesehen werden kann (vgl. Kahn, Curme 1987: 603). Firmen mit hohem mittleren Lohn beschäftigen im Durchschnitt Individuen mit höherem Humankapital als Organisationen mit niedrigem mittleren Lohn. Ist die Qualifikation der Arbeitnehmer/-innen in einer Organisation insgesamt sehr hoch, ist wahrscheinlich die Streuung des Humankapitals höher. Lazear und Shaw (2007) bringen dazu ein Beispiel: Eine Anwaltskanzlei benötigt auch Reinigungskräfte, eine Reinigungsfirma hingegen benötigt nicht notwendigerweise auch einen Anwalt. Eine weitere Erklärung für den positiven Zusammenhang ist, dass Firmen, die vermehrt hoch bezahlte Kräfte beschäftigen, eher der Tournament-Theorie ${ }^{65}$ folgen.

In Firmen mit durchschnittlich hohen Löhnen spielen Leistungsanreize eine größere Rolle als in Niedriglohn-Firmen. Lohnkompression würde bei hoch produktiven Kräften dazu führen, dass sie aufgrund adverser Selektion die Organisation verlassen (vgl. Lazear, Shaw 2007: 15). Ein dritter Grund ist statistischer Natur. Die Lohnstreuung ist meistens rechtsschief, d.h. nach unten hin ist die Höhe der Löhne begrenzt, nach oben jedoch offen. Während es am unteren Ende der Verteilung meistens eine Schranke in Form eines gesetzlichen oder tariflichen Mindestlohns gibt, unter den der Lohn nicht fallen darf, sind Löhnen nach oben hin keine Grenzen gesetzt. Firmen mit durchschnittlich hohen Löhnen haben demnach eine größere Streuung, da sie nach oben alle Werte annehmen kann, bei Firmen mit niedrigen Löhnen im Durchschnitt setzen jedoch Mindestlöhne oder Kollektivverträge eine Schranke.

Organisationen, die keinem Kollektivvertrag unterliegen oder sich nur an einem orientieren haben eine vermutete größere innerbetriebliche Lohnstreuung als Organisationen mit Kollektivvertrag, d.h. das erwartete Vorzeichen dieser beiden Dummys ist positiv, obwohl theoretisch auch das Gegenteil denkbar wäre (vgl. dazu Kapitel 10.2). Andere empirische Arbeiten bestätigen das vermutete positive Vorzeichen (vgl. Bechtel et al. 2005; Canal Dominguez, Rodriguez 
Gutierrez 2004; Dell'Aringa, Lucifora 1994; Gerlach, Stephan 2005; Gerlach, Stephan 2006a; Gosling, Machin 1995; Metcalf et al. 2000; Rodriguez Gutierrez 2001).

Die Organisationsgröße, gemessen an logarithmierten Vollzeit-äquivalenten (VZÄ) beeinflusst die Lohnstreuung vermutlich positiv, wobei auch hier das Gegenteil theoretisch denkbar wäre (vgl. dazu Kapitel 10.4). Empirisch zeigt sich in mehreren Studien, dass die Organisationsgröße positiv auf die Lohnstreuung wirkt (vgl. z.B.: Lallemand, Rycx 2006; Davis, Haltiwanger 1995; Haltiwanger, Davis 1996).

\section{Verwendete Daten und methodische Anmerkungen}

Bevor die Ergebnisse der Regressionsschätzung vorgestellt werden, werden Angaben zu den Kontrollvariablen gemacht und die Schätzmethode näher dargestellt.

\subsection{Beschreibung des Datensatzes und der Kontrollvariablen}

Wiederum werden die Daten des NPO-Organisationsdatensatzes der WU Wien und der Statistik Austria und Lohndaten aus der Steuerstatistik der Statistik Austria („NPO-Steuer-Daten 2006“) zusammengefügt. ${ }^{66}$ Im Unterschied zum vorangegangenen Teil werden für die Schätzung jedoch die LEE-Daten, bestehend aus Individual- und Organisations-daten auf Organisationsebene zusammengefasst. Aus den vorliegenden Individualdaten werden zuerst verschiedene Maße auf Organisations-ebene berechnet, d.h. z.B. der Mittelwert oder die Standardabweichung pro Organisation. Außerdem werden alle Fälle ausgeschlossen, in denen weniger als drei bezahlte Individeen tätig sind. Für Organisationen mit nur einer bezahlten Arbeitskraft kann logischerweise keine innerbetriebliche Lohnstreuung berechnet werden. Die Wahl von drei bezahlten Individuen ist willkürlich, weshalb die Berechnungen auch für alle Organisationen, mit mindestens zwei bezahlten Mitarbeiter/-inne/n und fünf Mitarbeiter/-inne/n durchgeführt wurden. $\mathrm{Da}$ sich an den wesentlichen Aussagen der Ergebnisse jedoch nichts ändert, werden die Ergebnisse nicht dargestellt. Folgende Tabelle 28 gibt einen Überblick über die Anzahl der Mitarbeiter/-innen pro Organisation.

61 Organisationen beschäftigen nur eine bezahlte Arbeitskraft. 43 Organisationen beschäftigen zwei Individuen. Ein überwiegender Teil der Organisationen beschäftigt weniger als 20 Mitarbeiter/-innen. Nur fünf NPO im Datensatz verzeichnen mehr als 1000 bezahlte Arbeitskräfte.

66 Für eine genauere Beschreibung des Datensatzes siehe Kapitel 7.3. 
Tabelle 28: Häufigkeit der Organisationen nach Anzahl der Mitarbeiter/innen

\begin{tabular}{|c|c|c|c|}
\hline $\begin{array}{c}\text { Anzahl } \\
\text { Mitarbeiter }\end{array}$ & Häufigkeit & $\begin{array}{c}\text { Anzahl } \\
\text { Mitarbeiter }\end{array}$ & Häufigkeit \\
\hline 1 & 61 & 36 & 2 \\
\hline 2 & 43 & 37 & 1 \\
\hline 3 & 37 & 40 & 2 \\
\hline 4 & 32 & 41 & 3 \\
\hline 5 & 18 & 44 & 1 \\
\hline 6 & 17 & 45 & 2 \\
\hline 7 & 9 & 46 & 1 \\
\hline 8 & 18 & 47 & 2 \\
\hline 9 & 7 & 48 & 2 \\
\hline 10 & 12 & 49 & 2 \\
\hline 11 & 10 & 50 & 2 \\
\hline 12 & 9 & 51 & 2 \\
\hline 13 & 8 & 52 & 1 \\
\hline 14 & 5 & 53 & 1 \\
\hline 15 & 6 & 57 & 1 \\
\hline 16 & 4 & 58 & 1 \\
\hline 17 & 10 & 59 & 1 \\
\hline 18 & 3 & 60 & 1 \\
\hline 19 & 3 & 61 & 2 \\
\hline 20 & 3 & 63 & 1 \\
\hline 21 & 3 & 72 & 1 \\
\hline 22 & 2 & 74 & 1 \\
\hline 25 & 2 & 75 & 3 \\
\hline 26 & 1 & 77 & 1 \\
\hline 27 & 6 & 78 & 1 \\
\hline 28 & 2 & 89 & 1 \\
\hline 29 & 1 & 94 & 1 \\
\hline 30 & 4 & $100-499$ & 31 \\
\hline 31 & 1 & $500-999$ & 4 \\
\hline 32 & 2 & $>1000$ & 5 \\
\hline 34 & 1 & \multirow{2}{*}{ Summe } & \multirow{2}{*}{421} \\
\hline 35 & 1 & & \\
\hline
\end{tabular}

Quelle: NPO-Steuer-Daten 2006, eigene Berechnungen

Folgende Variable werden für die Schätzung verwendet, über deren Mittelwerte und Standardabweichungen der verwendeten Variablen Tabelle 29 einen Überblick vermittelt. Die deskriptive Statistik wurde nur für Organisationen mit 2 oder mehr bezahlten Arbeitskräften erstellt: 
Tabelle 29: Deskriptive Statistik der verwendeten Variablen

\begin{tabular}{|c|c|c|c|c|c|}
\hline Variable & Anzahl & Durchschnitt & Standardabw. & Min. & Max. \\
\hline \multicolumn{6}{|c|}{ Lohnvariable } \\
\hline Standardab. Einkommen & 360 & 26,991 & 15,306 & 0 & 129,31 \\
\hline Standardabw. log. Einkommen & 360 & 0,591 & 0,296 & 0 & 1,631 \\
\hline $\mathrm{Q} 0,9 / \mathrm{Q} 0,1$ & 360 & 4,993 & 4,203 & 1 & 38,087 \\
\hline Varianz log. Einkommen & 360 & 0,437 & 0,403 & 0 & 2,66 \\
\hline $\begin{array}{l}\text { Logarithmen d. Standardabw. d. } \\
\text { Einkommen }\end{array}$ & 359 & 3,053 & 0,964 & $-3,783$ & 4,862 \\
\hline \multicolumn{6}{|c|}{ Zusammensetzung der Belegschaft } \\
\hline Standardabw. Alter & 360 & 8,756 & 3,592 & 0 & 21,92 \\
\hline Standardabw. Geschlecht & 360 & 0,346 & 0,229 & 0 & 0,707 \\
\hline $\begin{array}{l}\text { Standardabw. } \\
\text { Beschäftigungsausmaß }\end{array}$ & 360 & 0,299 & 0,233 & 0 & 0,707 \\
\hline Standardabw. soziale Stellung & 360 & 0,185 & 0,222 & 0 & 0,707 \\
\hline \multicolumn{6}{|c|}{ freiwillige Zuwendungen } \\
\hline $\begin{array}{l}\text { Standardabw. Ehrenamt } \\
\text { Anteil }\end{array}$ & 345 & 0,217 & 0,202 & 0 & 0,5 \\
\hline Spenden u.Sponsoring/ & 297 & 0,091 & 0,211 & 0 & 1 \\
\hline Gesamteinnahmen & & & & & \\
\hline $\begin{array}{l}\text { Anteil } \\
\text { Subvention/Gesamteinnahmen }\end{array}$ & 297 & 0,289 & 0,363 & 0 & 1 \\
\hline \multicolumn{6}{|c|}{ andere Organisationsvariablen } \\
\hline $\begin{array}{l}\text { Anteil Leistungsvertrag/ } \\
\text { Gesamteinnahmen }\end{array}$ & 297 & 0,212 & 0,326 & 0 & 1 \\
\hline ÖNACE 55 & 360 & 0,022 & 0,148 & 0 & 1 \\
\hline ÖNACE 73 & 360 & 0,031 & 0,172 & 0 & 1 \\
\hline ÖNACE 80 & 360 & 0,050 & 0,218 & 0 & 1 \\
\hline ÖNACE 85 & 360 & 0,403 & 0,491 & 0 & 1 \\
\hline ÖNACE 91 & 360 & 0,392 & 0,489 & 0 & 1 \\
\hline ÖNACE 92 & 360 & 0,103 & 0,304 & 0 & 1 \\
\hline durchschnittl. Lohnhöhe & 360 & 3,866 & 0,462 & 1,782 & 4,845 \\
\hline durchschnittl. geschätzte Lohnhöhe & 360 & 3,706 & 0,495 & 1,781 & 4,767 \\
\hline $\begin{array}{l}\text { kein Kollektivvertrag, aber } \\
\text { Orientierung }\end{array}$ & 323 & 0,328 & 0,470 & 0 & 1 \\
\hline kein Kollektivvertrag, keine & 323 & 031 & 0.467 & 0 & 1 \\
\hline Orientierung & 323 & 0,31 & 0,467 & 0 & I \\
\hline $\log . \mathrm{VZÄ}$ & 360 & 73,213 & 457,526 & 0,25 & 6449 \\
\hline
\end{tabular}

Quelle: NPO-Steuer-Daten 2006, eigene Berechnungen

In Tabelle 29 sind die jeweilige Fallzahl, der Mittelwert, die Standardabweichung sowie Minimum und Maximum der in der Regression verwendeten Variablen zu sehen. Im Fall des Streuungsmaßes und der durchschnittlichen Lohnhöhe werden jeweils alternative Variablen dargestellt. Von den drei vorkommenden Einnahmequellen - Subventionen, Einnahmen aus Leistungsverträgen mit der öffentlichen Hand sowie Spenden und Sponsoring - sind Subventionen die wichtigste Einnahmequelle. Im Durchschnitt stammen knapp 29\% der Ge- 
samteinnahmen aus Subventionen, gefolgt von $21 \%$, die aus Einnahmen aus Leistungsverträgen resultieren. $6 \%$ der Einnahmen kommen aus Spenden und knapp 3\% durch Sponsoring. Weitere, nicht in die Regression eingehende, Einnahmequellen einer NPO können etwa Einnahmen aus Umsätzen, Mitgliedsbeiträge oder Investitionszuschüsse sein.

Mehr als die Hälfte aller im Datensatz befindlichen Organisationen, nämlich ca. $62 \%$, haben ehrenamtliche Mitarbeiter/-innen. Die meisten Organisationen des Datensatzes sind in der Branche „Gesundheits-, Veterinär- und Sozialwesen“ tätig, gefolgt von der Branche „Erbringung von sonstigen öffentlichen und persönlichen Dienstleistungen", wobei die wichtigste Unterkategorie in dieser ÖNACE ,sonstige Interessensvertretungen und Vereine“ darstellt. Die restlichen Organisationen teilen sich auf die Branchen „Beherbungs- und Gaststättenwesen“ (ÖNACE 55), „Forschung und Entwicklung“ (ÖNACE 73), „Unterrichtswesen" (ÖNACE 80) und „Kultur, Sport und Unterhaltung“ (ÖNACE 92) auf. Bei der Frage, ob die NPO einem Kollektivvertrag unterliegt, teilen sich die Organisation etwa zu gleichen Teilen auf die drei Unterkategorien auf. Ein gutes Drittel unterliegt einem Kollektivvertrag, und jeweils nicht ganz ein Drittel unterliegt keinem, orientiert sich jedoch an einem, bzw. unterliegt keinem und orientiert sich auch an keinem Kollektivvertrag.

\subsection{Fehlende Werte}

Auch in diesem Datensatz liegen fehlende Werte vor. Folgende Tabelle 30 gibt an, bei welchen Variablen fehlende Werte auftreten. Variable, die nicht genannt werden, liegen im Datensatz vollständig vor:

Tabelle 30: Fehlende Werte im Datensatz

\begin{tabular}{l|c|c|c} 
Variable & \multicolumn{2}{c}{$\begin{array}{c}\text { vorhandene } \\
\text { Fälle in \% }\end{array}$} & $\begin{array}{c}\text { fehlende } \\
\text { Werte in \% }\end{array}$ \\
\hline vorhandene Fälle & $\mathbf{3 6 0}$ & & \\
Standardabweichung Ehrenamt & 345 & $95,83 \%$ & $4,17 \%$ \\
Anteil Subvention & 297 & $82,50 \%$ & $17,50 \%$ \\
Anteil Leistungsvertrag & 297 & $82,50 \%$ & $17,50 \%$ \\
Anteil Spenden/Sponsoring & 297 & $82,50 \%$ & $17,50 \%$ \\
kein Kollektivvertrag, aber Orientierung & 323 & $89,72 \%$ & $10,28 \%$ \\
kein Kollektivvertrag, keine Orientierung & 323 & $89,72 \%$ & $10,28 \%$ \\
\hline
\end{tabular}

Quelle: NPO-Steuer-Daten 2006, eigene Berechnungen

Zur Übersicht über die Arten fehlender Werte und den möglichen Umgang mit fehlenden Werten wird auf Kapitel 7.4 verwiesen. Auch hier wird davon ausgegangen, dass es sich bei den fehlenden Werten um Werte der Kategorie MAR (missing at random) handelt, d.h. die fehlenden Werte sind von einer anderen Variable im Datensatz abhängig. 
Zur Korrektur fehlender Werte werden die Berechnungen einerseits durch Fallausschluss, andererseits mit Missing Dummys angestellt. Im Ergebnisteil werden die Ergebnisse mit der Methode des Fallausschlusses präsentiert. Zusätzlich wird Tabelle 35 in Kapitel 15.2 einzelne Ergebnisse der Variablen, die fehlende Werte haben und deshalb mithilfe eines Missing Dummys berechnet wurden, zum Vergleich mit den Ergebnissen aus der Methode Fallausschluss präsentieren.

\subsection{Instrumentvariablenschätzung (IV Schätzung oder 2SLS- Schätzung)}

Die Instrumentvariablenschätzung (IV-Schätzung), die auch Hilfsvariablenschätzung, zweistufige OLS-Schätzung oder 2SLS (two-stage-least-squares)Schätzung genannt wird, wird in Fällen eingesetzt, in denen eine unabhängige Variable $x_{j}$ und der Fehlerterm $\varepsilon_{j}$ im Modell $y_{j}=x_{j}{ }^{\prime} \beta+\varepsilon_{j}$ nicht unkorreliert sind (vgl. Hackl, Glatzer 2000: $81 \mathrm{ff}$. und Greene 2003: 74ff.). Ist dies der Fall, so ist der OLS-Schätzer $b=\left(X^{\prime} X\right)^{-1} X^{\prime} y$ nicht konsistent. Zur Lösung des Problems wird die IV-Schätzung herangezogen. Dabei wird unterstellt, dass es eine

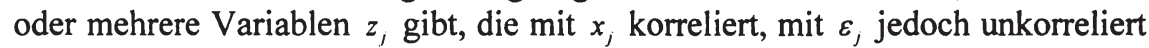
sind. Mit dem Kleinste-Quadrate-Schätzer kann $\beta$ nicht konsistent geschätzt werden, wenn $x$, und $\varepsilon_{j}$ korreliert sind, unter Verwendung von $z_{j}$ kann jedoch ein konsistenter Schätzer erlangt werden. Die Variablen $z_{j}$ werden verwendet, um in einer ersten Hilfsschätzung $\hat{x}_{j}$ zu schätzen. Diese Variable wird dann in der tatsächlichen Schätzung verwendet. Damit wird der sonst übliche Schätzer

(28) $b=\left(X^{\prime} X\right)^{-1} X^{\prime} y z u$

$$
b_{N}=\left(\hat{X}^{\prime} X\right)^{-1} \hat{X}^{\prime} y=\left[X^{\prime} Z\left(Z^{\prime} Z\right)^{-1} Z^{\prime} X\right]^{-1} X^{\prime} Z\left(Z^{\prime} Z\right)^{-1} Z^{\prime} y
$$

(vgl. Greene 2003: 78).

Dieser Schätzer ist konsistent und asymptotisch normalverteilt. Da der Schätzer $b_{l}$ dabei in zwei Schritten ermittelt wird, wird die Schätzung auch zweistufige Schätzung oder two-stage-least-squares genannt.

In der Praxis besteht die Schwierigkeit, geeignete Variablen zu finden, die als Instrumente dienen können. Die Variablen können z.B. aus theoretischen Überlegungen gefunden werden, bei Paneldaten eignen sich auch Werte aus Vorperioden.

In der vorliegenden Analyse wird das mittlere Einkommen instrumentiert, da ein Vorliegen dieses Problems vermutet wird. Ohne IV-Schätzung sind auch die Koeffizienten der anderen, nicht vom Endogenitätsproblem betroffenen Variablen verzerrt. 
Für die Instrumentierung des logarithmierten mittleren Einkommens wird das mittlere Einkommen durch verschiedene erklärende Variablen auf Individualebene und Organisationsebene geschätzt. Die Schätzung erfolgt mithilfe der Gleichung (10) aus dem Kapitel 6.2.

Dieselben Variablen, die die Streuung der Löhne in einer Organisation beeinflussen, haben auch Einfluss auf die mittlere Lohnhöhe einer Organisation. Damit wird der Vorgehensweise von Canal Dominguez und Rodriguez Gutierrez (2004) und Kahn und Curme (1987) gefolgt.

Der geschätzte Wert wird danach als unabhängige Variable zur Schätzung der innerbetrieblichen Lohnstreuung verwendet. Zur Berechnung des IV-Schätzers müssen die t-Statistiken nachträglich korrigiert werden, da sie nicht korrekt angezeigt werden (vgl. dazu Hackl, Glatzer 2000: 84).

\section{Ergebnisse}

Dieses Kapitel gibt einen kurzen Überblick über dekriptive Ergebnisse, danach werden die Forschungsfragen des zweiten Teils der Dissertation beantwortet.

\subsection{Deskriptive Ergebnisse}

Die folgende Tabelle gibt eine Übersicht über die innerbetriebliche Lohnspreizung im Datensatz, dargestellt anhand verschiedener Maßzahlen. Damit soll ein möglichst umfassendes Bild vermittelt werden.

Die ersten drei Reihen der Tabelle 31 enthalten zur Übersicht zunächst Angaben zum durchschnittlichen Bruttotageslohn einer Organisation, zum durchschnittlichen logarithmierten Bruttotageslohn und zum Medianlohn. Dargestellt sind jeweils der Mittelwert aller Mittelwerte (bzw. Mediane), sowie der Median der Mittelwerte (Mediane) und die Streuung der Mittelwerte (Mediane). D.h. es wurde z.B. ein durchschnittlicher Lohn pro NPO berechnet und in Folge der Durchschnitt über alle Organisationen berechnet. Der Wert des Medians ist dabei jeweils kleiner als der Mittelwert. Dies spiegelt die Tatsache wider, dass Löhne nach oben hin weiter streuen als nach unten hin, dass also die Löhne rechtsschief verteilt sind. Der Abstand $R$ gibt den Abstand zwischen höchstem und niedrigstem Lohn an. Außerdem wurden verschiedene Rangfolgen berechnet. Schließlich werden die Lohnstreuungsmaße Standardabweichung, Varianz und der Variationskoeffizient des Bruttotageslohns dargestellt. 
Tabelle 31: Lohnstreuung im Datensatz

\begin{tabular}{lrrr} 
N=360 & Mittelwert & $\begin{array}{r}\text { Standard- } \\
\text { Median }\end{array}$ & $\begin{array}{r}\text { abweichung } \\
\hline \text { Durschnittl. Lohn pro NPO }\end{array}$ \\
Durschnittl. log. Lohn pro NPO & 32,373 & 51,010 & 20,942 \\
Medianlohn pro NPO & 3,706 & 3,772 & 0,495 \\
Abstand R & 49,496 & 46,310 & 22,084 \\
Q0,9/Q0,5 & 95,040 & 78,572 & 76,326 \\
Q0,75/Q0,25 & 1,938 & 1,622 & 1,252 \\
Q0,5/Q0,1 & 2,695 & 1,984 & 2,015 \\
Q0,9/Q0,1 & 2,590 & 1,997 & 1,748 \\
Variationskoeffizient d. Lohns & 4,993 & 3,554 & 4,204 \\
Standardabw. d. Lohns & 0,530 & 0,501 & 0,252 \\
Varianz d. Lohns & 26,991 & 25,499 & 15,306 \\
\hline
\end{tabular}

Quelle: NPO-Steuer-Daten 2006, eigene Berechnungen

Folgende Tabelle 32 zeigt jeweils deskriptive Ergebnisse für die innerbetriebliche Lohnstreuung, die für nonprofit-spezifischen Variablen getrennt berechnet wurden. Als Lohnstreuungsmaß wurde die Standardabweichung des Tageseinkommens pro Organisation verwendet. Bei den nonprofit-spezifischen Variablen wurden die Organisationen jeweils in zwei Gruppen geteilt: eine Gruppe, die Werte unterhalb des Medians der jeweiligen Variable annimmt, die zweite beinhaltet Werte über dem Median. Dargestellt werden jeweils die mittlere Lohnstreuung und die Standardabweichung der Lohnstreuung.

Auffallende Unterschiede in den Mittelwerten der Standardabweichung des Tageseinkommens pro Organisation sind vor allem bei der Unterscheidung der Organisationen hinsichtlich der ehrenamtlichen Mitarbeiter/-innen und des Anteils der Spenden und des Sponsorings zu finden. So ist - dem ersten Eindruck nach - die Lohnstreuung in Organisationen, die wenig freiwilligen Mitarbeiter/innen im Vergleich zu bezahlten Mitarbeiter/-innen haben, größer als in jenen mit überdurchschnittlich vielen. Organisationen, die in dieser Hinsicht über dem Median liegen, haben eine deutlich geringere Lohnstreuung. Organisationen mit einem niedrigen Spenden- und Sponsoringanteil haben eine höhere Lohnstreuung als jene, die einen hohen Anteil an Spenden an den Gesamteinnahmen verzeichnen. Hinsichtlich der Subventionsvariable sind die Unterschiede im Mittelwert der Lohnstreuung wesentlich geringer.

Die Unterschiede im Feld „Mittelwert Total“ in den einzelnen Tabellenabschnitten erklären sich daraus, dass je nach fehlenden Werten jeweils eine andere Fallzahl bzw. unterschiedliche Organisationen in die Berechnung eingehen. In die Berechnung des Feldes Mittelwert Total im Abschnitt ,Standardabwei- 
chung Ehrenamt' gehen etwa nur Organisationen ein, die angaben, wieviele ehrenamtliche Mitarbeiter/-innen sie beschäftigen.

Tabelle 32: Kreuztabellen

\begin{tabular}{|c|c|c|c|}
\hline \multirow[b]{2}{*}{ Standardabw. Ehrenamt } & \multicolumn{3}{|c|}{ Standardabweichung Tageseinkommen } \\
\hline & Mittelwert & Standardabw. & Häufigkeit \\
\hline unter Median & 29,601 & 15,641 & 173 \\
\hline über Median & 24,094 & 13,253 & 172 \\
\hline \multirow[t]{2}{*}{ Total } & 26,855 & 14,739 & 345 \\
\hline & \multicolumn{3}{|c|}{ Standardabweichung Tageseinkommen } \\
\hline Anteil Spenden/ Sponsoring & Mittelwert & Standardabw. & Häufigkeit \\
\hline unter Median & 28,705 & 15,595 & 149 \\
\hline über Median & 25,909 & 14,839 & 148 \\
\hline \multirow[t]{2}{*}{ Total } & 27,312 & 15,261 & 297 \\
\hline & \multicolumn{3}{|c|}{ Standardabweichung Tageseinkommen } \\
\hline Anteil Subventionen & Mittelwert & Standardabw. & Häufigkeit \\
\hline unter Median & 27,685 & 16,968 & 149 \\
\hline über Median & 26,936 & 13,374 & 148 \\
\hline Total & 27,312 & 15,261 & 297 \\
\hline
\end{tabular}

Quelle: NPO-Steuer-Daten 2006, eigene Berechnungen

Durch eine Regression unter Einbezug anderer erklärender Variablen ist nun zu prüfen, ob die oben aufgezeigten Unterschiede auch nach Kontrolle anderer Unterschiede der Organisationen signifikant sind.

\subsection{Regressionsergebnisse}

Im Folgenden werden die Ergebnisse verschiedener Regressionsschätzungen zur Beantwortung der Forschungsfragen vorgestellt. Dazu wurden verschiedene Modellvarianten mit unterschiedlichen Lohnstreuungsmaßen als abhängige Variable und verschiedenen erklärenden Variablen geschätzt. Der Grund für diese Vielzahl an Varianten besteht einerseits darin, die Robustheit der Ergebnisse bei Wahl unterschiedlicher Kontrollvariable zu überprüfen, andererseits wird damit die Vielfalt an verwendeten Lohnstreuungs-maßen in der vorhandenen empirischen Literatur ausgedrückt. Die Berechnung der Schätzungen erfolgte wiederum mit dem Statistikprogramm STATA 9.2.

Tabelle 33 zeigt die Ergebnisse einer Schätzung mit sämtlichen oben angeführten Kontrollvariablen (,Langvariante“) und vier verschiedenen abhängigen Variablen: der Standardabweichung des logarithmierten Bruttotageseinkommens, dem Q0,9/Q0,1- Quotienten, der Varianz des logarithmierten Bruttotageseinkommens und mit dem Logarithmus der Standardabyeichungen der Brutto- 
tageseinkommen. Das Verwenden von Varianzen im Vergleich zu Standardabweichungen hat zur Folge, dass Löhne, die besonders hoch oder niedrig sind d.h. hohe Abweichungen vom Mittelwert haben, stärker gewichtet werden. Tabelle 34 zeigt in Folge die Ergebnisse eines ,verkürzten“ Modells, in dem vor allem jene Kontrollvariable ausgeschlossen wurden, die sich im ausführlichen Modell als insignifikant erwiesen. Abschließend stellt Tabelle 36 einen Vergleich wesentlicher Ergebnisse aus der Variante unter Verwendung des Fallausschlusses und unter Einbezug von Missing Dummys dar.

Tabelle 33 zeigt die Ergebnisse von IV-Schätzungen unter Verwendung unterschiedlicher abhängiger Variablen. Vorwegzuschicken ist, dass unter ,Streuung' (Alter, Geschlecht etc.) - außer in der Schätzung auf die Varianz der logarithmierten Löhne - die Standardabweichung gemeint ist, im Fall mit der Varianz der logarithmierten Bruttotageslöhne jeweils die Varianz. Unter durchschnittlicher Lohnhöhe ist jeweils - mit einer Ausnahme - der geschätzte Mittelwert der logarithmischen Bruttotageslöhne pro Organisation gemeint. Im letzten Modellfall, wo der Logarithmus der Standardabweichung der Löhne die abhängige Variable darstellt, ist jedoch der Logarithmus der geschätzten mittleren Bruttotageslöhne gemeint.

Ein Vergleich der Ergebnisse zwischen den Modellen zeigt, dass sich die Ergebnisse nicht grundlegend voneinander unterscheiden. Mit einer Ausnahme weisen alle signifikanten Koeffizienten dieselben Vorzeichen auf. Zum Teil unterscheiden sich die Varianten in den Signifikanzen. Das korrigierte $\mathbf{R}^{2}$ liegt für die Schätzungen zwischen 0,13 (im vierten Modellfall) und 0,26 (im dritten Modellfall).

Das Ergebnis für die Ehrenamtsvariable entspricht dem erwarteten negativen Vorzeichen, Spenden und Sponsoring sowie Subventionen haben ein positives Vorzeichen. Spenden und Subventionen erlauben es der NPO, Löhne stärker zu variieren.

Die Standardabweichung der logarithmierten Bruttotageslöhne ist in Organisationen, in denen das Verhältnis zwischen bezahlten Vollzeit-äquivalenten und ehrenamtlichen Mitarbeiter/-inne/n aus-geglichener ist, kleiner als in Organisationen mit entweder wenig Freiwilligen im Vergleich zu bezahlten Arbeitskräften oder sehr vielen ehrenamtlichen Mitarbeiter/-innen. Steigt beispielsweise die Standardabweichung der ehrenamtlichen Mitarbeiter/-innen um 1, so sinkt die Standardabweichung der logarithmierten Bruttotageslöhne um 0,24. 


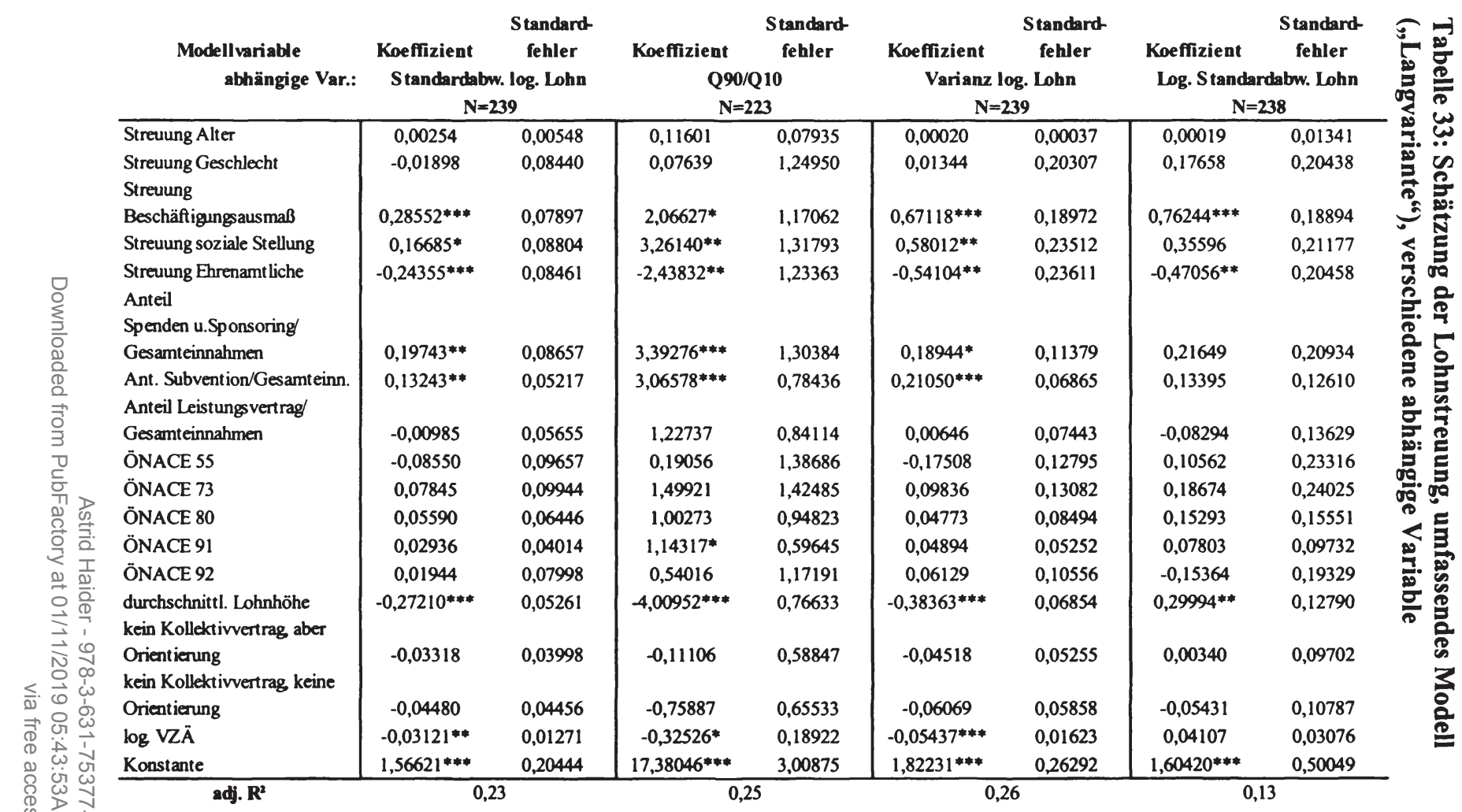

*** $\left({ }^{* *}\right)|*|$ auf $99 \%(95 \%)[90 \%-\mid$ Level signifiksnt

Quelle: NPO-Steuer-Daten 2006, eigene Berechnungen 
Ein hoher Anteil an Spenden und Sponsoring an den Gesamteinnahmen erhöht die Lohnstreuung. Nur im letzten Modellfall weist diese Variable keine signfikant von null verschiedenen Ergebnisse auf. Wird der Anteil an Spenden und Sponsoring an den Gesamteinnahmen um einen Prozent-punkt erhöht, erhöht sich die Standardabweichung der logarithmierten Bruttotageslöhne um 0,19.

Höhere Subventionen führen, zumindest in den ersten drei Modellvarianten, ebenfalls zu höherer Lohnstreuung. Hier steigt die Lohnstreuung um 0,13 bei einer Erhöhung des Anteils der Subventionen um einen Prozentpunkt. Der Anteil der Leistungsverträge an den Gesamteinnahmen hat keinen signifikanten Einfluss auf die Lohn-streuung.

Die Anzahl der Vollzeitäquivalente als Maß der Organisationsgröße hat ebenfalls in den ersten drei Modellvarianten einen signifikanten Einfluss. Anders als erwartet ist das Vorzeichen jedoch negativ, d.h. mehr bezahlte Beschäftigte führen zu einer niedrigeren Lohnstreuung. Dieses Ergebnis kann vielleicht damit erklärt werden, dass in Organisationen mit nur wenigen bezahlten Mitarbeiter/innen sehr unterschiedliche Individuen beschäftigt werden bzw. sehr unterschiedliche Positionen, wie zum Beispiel ein bezahlter Geschäftsführer bzw. eine bezahlte Geschäftsführerin und eine administrative Kraft, besetzt werden. In NPO mit vielen bezahlten Beschäftigten könnten hingegen viele Personen mit der gleichen Tätigkeit und einer ähnlichen Bezahlung beschäftigt sein.

Bei anderen Kontrollvariablen die aufgenommen wurden, um weitere Unterschiede zwischen den Organisationen zu beschreiben, ergeben sich keine signifikanten Ergebnisse. Weder die Branche noch die Frage, ob sich die Organisation an einem Kollektivvertrag orientiert bzw. keinem Kollektivvertrag unterliegt, haben einen von null verschiedenen Einfluss auf die erwartete Lohnstreuung einer Organisation. Vielleicht spiegelt der fehlende Einfluss der Kollektivverträge auf die Lohnhöhe den geringen Stellenwert von Gewerkschaften im Nonprofit Sektor wider. Ein Branchendummy (,ÖNACE 91') ${ }^{67}$ hat in einem Modellfall ein signifikant von null verschiedenes Ergebnis, was jedoch nicht als robustes Ergebnis eingeschätzt werden kann.

Bei den Variablen, die die unterschiedliche Zusammensetzung der Arbeitnehmer/-innen in einer Organisation beschreiben, sind die Streuung der Angestellten und der Vollzeitkräfte signifikant positiv, was dem erwarteten Vorzeichen entspricht, die Streuung des Alters und die des Geschlechts sind hingegen nicht signifikant. Höhere Standard-abweichungen der Zahl der Angestellten und der Zahl der Vollzeitkräfte erhöhen die Lohnstreuung.

67 Das sind Interessenvertretungen, kirchliche und sonstige religiöse Vereinigungen, sonstige Vereine (ohne Sozialwesen, Kultur und Sport). 
Die durchschnittliche Lohnhöhe weist unterschiedliche Ergebnisse auf. Für die ersten drei Modellvarianten hat die durchschnittliche Lohnhöhe einen signifikant negativen Einfluss auf die Lohnstreuung, im letzten Fall einen signifikant positiven. Das negative Vorzeichen, d.h. höhere durchschnittliche Löhne führen zu einer geringeren Lohnstreuung, entspricht nicht dem erwartenten. Die unterschiedlichen Vorzeichen je nach verwendetem Streuungsmaß für die abhängige Variable zeigen aber, dass das Ergebnis als nicht sehr robust eingeschätzt werden kann.

Tabelle 34 weist zur Überprüfung der Robustheit der oben dargestellten Ergebnisse, Koeffizienten und Standardfehler für eine andere Spezifikation der Schätzgleichung auf. Hier wurden die Branche, die Kollektivvertragsdummys und die Anzahl der Vollzeitäquivalente nicht in die Schätzgleichung aufgenommen. Die Fallzahl ist nun höher, was damit erklärt werden kann, dass durch eine geringere Anzahl fehlender Werte mehr NPO in die Berechnungen eingeschlossen wurden.

Im Vergleich der Ergebnisse mit jenen der „Langvariante“ fällt auf, dass sowohl die Vorzeichen der Koeffizienten gleich geblieben sind als auch die Signifikanzen annähernd dieselben sind. Gegenüber der „Langvariante“ verbessert sich das korrigierte $\mathrm{R}^{2}$ für den vierten Modellfall sogar um 4 Prozentpunkte, für die erste Variante bleibt das korrigierte $\mathrm{R}^{2}$ gleich, für die mittleren beiden Fälle verschlechtert es sich geringfügig. Auch die Höhe der Koeffizienten kann als ähnlich eingestuft werden. Insgesamt kann aus dem Vergleich geschlossen werden, dass die Ergebnisse sehr robust gegenüber Änderungen in der genauen Modellspezifikation sind und keine dahinterliegenden Variablen auf die in den Regressionen verwendeten Variablen mehr wirken.

Wieder ist der Koeffizient für die Ehrenamtsvariable negativ, allerdings weist der zweite Modellfall kein signifikantes Ergebnis auf. Die Variable ,Spenden und Sponsoring/Gesamteinnahmen' ist wiederum positiv, auch höhere Subventionen erhöhen die Lohnstreuung. Auch hier weist ein Modellfall - der vierte keine signifikant von null verschiedenen Ergebnisse auf.

Die Koeffizienten der Variable ,Streuung soziale Stellung' weisen im Gegensatz zur Variante mit mehr Kontrollvariablen nur mehr zwei signifikante Ergebnisse auf. Die ,Streuung des Beschäftigungsausmaßes' hat annähernd die gleichen Werte als in der „Langvariante“, was dafür spricht, dass diese Ergebnisse als robust einzuschätzen sind.

Die Koeffizienten der , durchschnittlichen Lohnhöhe' sind wiederum für die ersten drei Modellfälle negativ, für den letzten Fall positiv. 
IV

Modellvariable Koeffizient

Standard-

abhängige Var.: Standardabw. log. Lohn

Koeffizient

tandard

IV

Standard-

IV

Standard-

둘

Q90/Q10

$\mathrm{N}=\mathbf{2 4 0}$

Varianz log. Lohn

fehler

Log. Standardabw. Lohn

\begin{tabular}{|c|c|c|c|c|c|c|c|c|}
\hline & \multicolumn{2}{|c|}{$\mathrm{N}=\mathbf{2 5 7}$} & \multicolumn{2}{|c|}{$\mathrm{N}=240$} & \multicolumn{2}{|c|}{$\mathrm{N}=257$} & \multicolumn{2}{|c|}{$\mathrm{N}=256$} \\
\hline Streuung Alter & $-0,00073$ & 0,00508 & 0,05229 & 0,07525 & $-0,00005$ & 0,00035 & 0,00206 & 0,01203 \\
\hline Streuung Geschlecht & 0,03959 & 0,07751 & 0,42845 & 1,18561 & 0,19789 & 0,18818 & 0,20842 & 0,18150 \\
\hline Streuung Besch.ausmaß & $0,28315^{* * *}$ & 0,07124 & $2,236^{* *}$ & 1,08152 & $0,69148^{* * *}$ & 0,17668 & $0,87708^{* * *}$ & 0,16642 \\
\hline Streuung soziale Stellung & 0,08953 & 0,07576 & $2,80638^{* *}$ & 1,16842 & 0,26710 & 0,20175 & $0,33702^{*}$ & 0,17595 \\
\hline Streuung Ehrenamtliche & $-0,20988^{* * *}$ & 0,07783 & $-1,65459$ & 1,16239 & $-0,39898^{*}$ & 0,22203 & $-0,52481^{* * *}$ & 0,18247 \\
\hline Anteil & & & & & & & & \\
\hline $\begin{array}{l}\text { Spenden u.Sponsoring } \\
\text { Gesamteinnahmen }\end{array}$ & $0,19224^{* *}$ & 0,07750 & $3,43053^{* * *}$ & 1,19791 & $0,19915^{*}$ & 0,10396 & 0,17071 & 0,18167 \\
\hline $\begin{array}{l}\text { Ant. Sub- } \\
\text { vention/Gesamteinn. }\end{array}$ & $0,14484^{* * *}$ & 0,04937 & $2,88282^{* * *}$ & 0,75211 & $0,22783^{* * *}$ & 0,06659 & 0,09493 & 0,11550 \\
\hline $\begin{array}{l}\text { Anteil Leistungsvertrag/ } \\
\text { Gesamteinnahmen }\end{array}$ & $-0,04289$ & 0,05278 & 0,46278 & 0,80273 & $-0,04480$ & 0,07086 & $-0,09296$ & 0,12308 \\
\hline durchschnittl Lohnhöhe & $-0,26617^{* * *}$ & 0,04837 & $-3,80913^{* * *}$ & 0,72223 & $-0,38473^{* * *}$ & 0,06456 & $0,39917^{* * *}$ & 0,11357 \\
\hline Konstante & $1,48533^{* * *}$ & 0,18740 & $16,7586^{* * *}$ & 2,82085 & $1,69440^{* * *}$ & 0,24618 & $1,31923^{* * *}$ & 0,44408 \\
\hline
\end{tabular}

adj. $R^{2}$

0,23

0,24

*** $\left.{ }^{(* \star}\right)\left[{ }^{\star}\right]$ auf $99 \%(95 \%)$ [90\%-] Level signifikant

Quelle: NPO-Steuer-Daten 2006, eigene Berechnungen 
Abschließend wird ein Vergleich der Ergebnisse, die unter Verwendung des Fallausschlusses und unter Verwendung von Missing Dummys berechnet wurden, angestellt. Dargestellt sind die Ergebnisse für die Standardabweichung der logarithmierten Bruttotageseinkommen als der abhängigen Variable. Die Version mit Missing Dummys wurde derart berechnet, dass alle fehlenden Werte auf Null gesetzt wurden und gleichzeitig jeweils ein Dummy eingefügt wurde, der den Wert 1 annimmt, wenn der Wert fehlend ist und den Wert 0 , wenn er nicht fehlend ist. Im Fall des Ausschlusses, gingen Datenfälle mit fehlenden Werten nicht in die Berechnung mit ein, weshalb die Fallzahl hier kleiner ist.

Tabelle 35: Ergebnisse der Schätzung auf die Lohnstreuung bei Verwendung unterschiedlicher Methoden zur Korrektur fehlender Werte

IV

Standard-

Modellvariable

Koeffizient
IV

Standard-

fehler abhängige Variable: Standardabw. der logarithm. Bruttotageseinkommen

$\mathbf{N}=\mathbf{3 1 7}$

$\mathrm{N}=\mathbf{2 3 9}$

Missing Dummy

\begin{tabular}{|c|c|c|c|c|}
\hline $\begin{array}{l}\text { Standardabw. Ehrenamt } \\
\text { Standardabw. Ehrenamt }\end{array}$ & $-0,21750^{* * *}$ & 0,07834 & $-0,24355^{* * *}$ & 0,08461 \\
\hline Missing Dummy & $-0,05950$ & 0,08113 & $\cdots$ & $\cdots$ \\
\hline $\begin{array}{c}\text { Spenden u. Sponsoring/Gesamt- } \\
\text { einnahmen }\end{array}$ & $0,18276^{* *}$ & 0,08324 & $0,19743^{* *}$ & 0,08657 \\
\hline $\begin{array}{c}\text { Subvention/Gesamt-einnahmen } \\
\text { Leistungsvertrag/ }\end{array}$ & $0,12499^{* *}$ & 0,05202 & $0,13243^{* *}$ & 0,05217 \\
\hline $\begin{array}{c}\text { Gesamteinnahmen } \\
\text { Finanzierungsstruktur Missing }\end{array}$ & $-0,01680$ & 0,05673 & $-0,00985$ & 0,05655 \\
\hline $\begin{array}{c}\text { Dummy } \\
\text { kein Kollektivvertrag, aber }\end{array}$ & $-0,05154$ & 0,04762 & --- & $\ldots$ \\
\hline $\begin{array}{c}\text { Orientierung } \\
\text { kein Kollektivvertrag, keine }\end{array}$ & $-0,00249$ & 0,03839 & $-0,03318$ & 0,03998 \\
\hline Orientierung & $-0,00564$ & 0,04213 & $-0,04480$ & 0,04456 \\
\hline KV Missing Dummy & 0,04034 & 0,05736 & -- & $\cdots$ \\
\hline Konstante & $1,20991^{* * *}$ & 0,13869 & $1,56621^{* * *}$ & 0,20444 \\
\hline
\end{tabular}

Quelle: NPO-Steuer-Daten 2006, eigene Berechnungen

Es zeigt sich, dass keine Missing Dummy Variable signifikante Ergebnisse aufweist. Alle Koeffizienten zeigen dieselben Vorzeichen und haben annähernd dieselbe Höhe. Auch hier kann folglich festgestellt werden kann, dass die Ergebnisse unabhängig von der Wahl der Methode im Umgang mit fehlenden Variablen als robust eingeschätzt werden können. 


\subsection{Beantwortung der Forschungsfragen}

Der zweite Teil der Disseration stellte die Frage, welche Determinanten Einfluss auf die innerbetriebliche Lohnstreuung einer Organisation haben. Hinsichtlich der nonprofit-spezifischen Elemente zeigt sich, dass sich das Verhältnis von ehrenamtlichen Mitarbeiter/-innen und bezahlten Vollzeitäquivalenten auswirkt. Je ausgeglichener das Verhältnis von freiwilligen Mitarbeiter/-inne/n zu bezahlten Arbeitskräften in einer Organisation ist, desto geringer ist die Lohnstreuung. Steigt beispiels-weise die Standardabweichung der ehrenamtlichen Mitarbeiter/innen um 1, so sinkt die Standardabweichung der logarithmierten Löhne um 0,21 bzw. 0,24, je nach gewählten Kontrollvariablen. Auch für die anderen Modellfälle, für die unterschiedliche Streuungsmaße als abhängige Variable verwendet wurden, zeigt sich ein ähnliches Bild.

Der Anteil von Spenden und Sponsoring an den Gesamteinnahmen weist in fast allen Modellvarianten ein signifikant positives Ergebnis auf. Eine NPO, die sich zu großen Teilen aus Spenden und Sponsoring finanziert, hat also eine höhere Lohnstreuung als eine Organisation, bei der das nicht der Fall ist. Ähnlich verhält es sich bei Subventionen. Auch hier liegt ein signifikant positives Ergebnis vor, das Ergebnis trifft auf drei von vier Modellspezifikationen zu. Eine hohe Abhängigkeit von Subventionen vergrößert demnach tendenziell die Lohnstreuung. In der Modellspezifikation mit den Logarithmen der Standardabweichungen der Bruttotageslöhne als abhängige Variable sind die Koeffizienten der beiden Variablen (Spenden/Sponsoring und Subventionen) nicht signifikant von 0 verschieden.

Kontrollvariablen, die über mehrere oder alle Modellvarianten hindurch signifikanten Einfluss auf die innerbetriebliche Lohnstreuung haben, sind die Streuung der Angestellten, der Vollzeitbeschäftigten, die logarithmierten Vollzeitäquivalente und die mittlere Lohnhöhe der NPO. Anders als erwartet, sind die Vorzeichen der beiden letzten Variablen in den meisten Modellfällen negativ, d.h. mehr bezahlte Beschäftigte und ein höherer Durchschnittslohn in einer NPO verringern die Lohn-streuung. In der letzten präsentierten Spezifikation ist der Koeffizient des Logarithmus der durchschnittlichen Löhne wiederum positiv und entspricht damit dem erwarteten Vorzeichen. Insgesamt sind diese Ergebnisse als nicht sehr robust einzuschätzen. Je nach genauer Spezifikation ändern sich für diese Variablen die Ergebnisse.

Die Branche, in der die Organisation aktiv ist, sowie die Frage, ob eine Organisation gemäß eines Kollektivvertrags bezahlt, weisen dagegen - außer in einem Fall - durchgehend keine signifikanten Ergebnisse auf. Nur in einem Fall wies der Sektor ,Interessensvertretungen (ÖNACE 91)' ein signifikant positives Vorzeichen auf, was ebenfalls nicht als robustes Ergebnis eingeschätzt werden kann.

Die Unterschiede in den Ergebnissen mit Bezug auf die Signifikanzniveaus und die Stärke der Effekte, die durch unterschiedliche Spezifikationen der Variablen 
hervorgerufen sind, zeigen in der Gesamtbeurteilung, wie wichtig eine Diskussion um die Verwendung einzelner Maße ist. In der vorhandenen empirischen Literatur werden Unterschiede durch verschiedene Streuungsmaße überraschend wenig diskutiert, obwohl, wie eben gezeigt wurde, das Ergebnis dadurch in Teilen beeinflusst wird. 
Astrid Haider - 978-3-631-75377-4

Downloaded from PubFactory at 01/11/2019 05:43:53AM

via free access 
Teil III:

Zusammenfassende Schlussbetrachtung und Ausblick 
Astrid Haider - 978-3-631-75377-4

Downloaded from PubFactory at 01/11/2019 05:43:53AM

via free access 
Die Dissertation hatte zum Ziel, Besonderheiten von NPO in die Lohnanalysen miteinzubeziehen. Es wurde der Einfluss ehrenamtlicher Arbeit, von Spenden und von Subventionen auf die Lohnhöhe und die innerbetriebliche Lohnstreuung in NPO untersucht.

Ziel des ersten Teils der Dissertation war es, eine Lohnfunktion für NPO zu schätzen. Spezielle Beachtung fanden dabei (freiwillige) Zuwendungen, in Form von freiwilliger Arbeit, Spenden und Subventionen an NPO, die das Budget der Organisation erweitern. Die zwei Forschungsfragen des Abschnitts lauteten: Beeinflussen Ehrenamtlichkeit, Spenden und Subventionen die Lohnhöhe von bezahlten Arbeitnehmer/-innen in NPO? Wenn diese Elemente eine Rolle bei der Bestimmung der Lohnhöhe spielen, auf welche Art und Weise beeinflussen sie die Lohnhöhe?

Zur Beantwortung der Forschungsfragen wurden einerseits theoretische Ansätze zur Erklärung der Lohnhöhe in der Arbeitsmarkttheorie kurz vorgestellt, andererseits Besonderheiten des Nonprofit Sektors in Hinsicht auf die vorgestellten Ansätze erläutert. Dabei wurde speziell auf die Einflussgrößen, die in den Forschungsfragen genannt sind - ehrenamtliche Arbeit, Spenden und Subventionen - eingegangen. Es zeigte sich, dass Argumente sowohl für einen positiven Einfluss dieser drei Variablen auf die Lohnhöhe als auch für einen negativen existieren.

Zusätzlich wurden vorhandene empirische Studien, die sich mit der Lohnhöhe von bezahlten Arbeitnehmer/-innen in NPO beschäftigen, zusammengefasst. Dabei war festzustellen, dass sich die meisten Studien mit einem Vergleich der Lohnhöhe im Nonprofit Sektor mit jener im gewinnorientierten Sektor befassen, wobei ein Großteil der Analysen US-amerikanische Daten verwendet. Nur wenige Arbeiten verwenden sogenannte LEE-Daten, die Angaben der Individuen und der Or-ganisationen beinhalten. Insgesamt zeigt sich, dass aus den vorhandenen Analysen kein Schluss gezogen werden kann, in welchem Sektor die Löhne höher sind. Ein Grund der Unterschiede in den Ergebnissen liegt vermutlich in der Vielfalt der Daten, der Datenebenen und der verwendeten Kontrollvariablen.

In der vorliegenden Arbeit stand Unterschiede innerhalb des Nonprofit Sektors im Mittelpunkt. Die empirische Schätzung der Lohnfunktion erfolgte anhand der LEE-Daten „NPO-Steuer-Daten 2006“, die In-formationen zu 39.613 Arbeitnehmer/-innen in 421 österreichischen NPO umfassen. Zur Schätzung wurde einerseits eine OLS-Regression durchgeführt, andererseits eine Quantilsregression, die den Vorteil hat, dass der Einfluss der unabhängigen Variablen auf verschiedenen Quantilen der abhängigen Variablen geschätzt werden kann. Diese Methode wurde bislang noch nicht zur Schätzung der Lohnhöhe im Nonprofit Sektor verwendet. 
Die Ergebnisse zeigen, dass Organisationen mit ehrenamtlichen Mitarbeiter/innen weniger hohe Löhne zahlen als Organisationen ohne freiwillige Arbeitskräfte, wobei die Größe des Koeffizienten ca. $10 \%$ beträgt und an den Rändern der Lohnverteilung weniger stark ausgeprägt ist. Die konkrete Anzahl der ehrenamtlichen Mitarbeiter/-innen hat keinen Einfluss. Während in der OLSRegression Spenden keinen signifikanten Einfluss auf die Lohnhöhe haben, zeigt sich bei der Quantilsregression, dass sie bei hohen Löhnen einen positiven Koeffizienten aufweisen. Subventionen haben bei beiden Schätz-methoden einen signifikanten positiven Einfluss auf die Lohnhöhe, wobei dieser bei hohen Löhnen stärker ist.

Der zweite Teil der Dissertation beschäftigt sich, in Vertiefung des ersten Teils mit der innerbetrieblichen Lohnstreuung im Nonprofit Sektor. Dabei wird die Frage gestellt, ob freiwillige Arbeit, Spenden und Subventionen die innerbetriebliche Lohnstreuung von NPO beeinflussen.

Zur Beantwortung der Forschungsfragen wurden wiederum zuerst allgemein Theorien über die innerbetriebliche Lohnstreuung vorgestellt, bevor auf Besonderheiten im Nonprofit Sektor eingegangen wurde. Ein Exkurs behandelte Löhne von Führungskräften in NPO, da sie einerseits die innerbetriebliche Lohnstreuung als „Ausreißer“ besonders be-einflussen und sie andererseits in verstärktem Ausmaß als Vergleichs-werte für die Einschätzung der Angemessenheit des Lohns für ein Individuum dienen.

Das nächste Unterkapitel der Dissertation stellte die Ergebnisse und die Methoden vorhandener empirischer Studien vor, wobei wiederum gesondert auf NPO eingegangen wurde. Dabei wurde festgestellt, dass zur innerbetrieblichen Lohnstreuung in NPO keine empirischen Arbeiten vorliegen.

Kapitel 13 stellt den für die empirische Analyse verwendeten Modellrahmen vor. Im Zuge dessen wurden auch verschiedene Berechnungsmethoden der Lohnstreuung dargestellt und die einzelnen Variablen spezifiziert. Zur Berechnung der Determinanten der innerbetrieblichen Lohnstreuung in NPO wurden wiederum die NPO-Steuerdaten 2006 verwendet. Die NPO Daten wurden vom Institut für Sozialpolitik gemeinsam mit der Statistik Austria erhoben und mit Individualsteuerdaten aus der Steuerstatstik durch die Statistik Austria ergänzt. Anders als im ersten Teil der Dissertation wurden die LEE-Daten auf Organisationsebene aggregiert. Außerdem wurden nur Organisationen in die Schätzungen einbezogen, die mehr als zwei bezahlte Individuen beschäftigten. Allerdings wurden die Schätzungen zur Überprüfung der Robustheit der Ergebnisse auch mit anderen Fallzahlen (Organisationen mit mehr als einer bezahlten Arbeitskraft und mit mehr als vier bezahlten Arbeitskräften) wiederholt. Die Grundaussagen ändern sich dadurch nicht.

Für die Schätzungen wurden verschiedene Streuungsmaße herangezogen, nämlich die Standardabweichung der logarithmierten Löhne, der $Q 0,9 / Q 0,1$-Quo- 
tient, die Varianz der logarithmierten Löhne und die Logarithmen der Standardabweichungen der Bruttotageslöhne, da sich, wie aus der vorhandenen Literatur ersichtlich, empirisch kein Maß durch besondere Vorteile auszeichnet und sich in der Empirie auch kein Maß als gängig etabliert hat. Durch die Verwendung unterschiedlicher Maße konnte auch die Robustheit der Ergebnisse überprüft werden. Zur Schätzung wurde eine Instrumentvariablenschätzung herangezogen, da bei Einbezug der mittleren Lohnhöhe in einer Organisation als Kontrollvariable ein Endogenitätsproblem vorliegt.

Die Ergebnisse zeigen, dass nonprofit-spezifische Variablen wie ehrenamtliche Beschäftigte, Spenden und Subventionen die Lohnstreuung beeinflussen. Organisationen, die ein ausgeglichenes Verhältnis zwischen bezahlten Arbeitskräften und freiwillige Mitarbeiter/-inne/n haben, haben eine geringere Lohnstreuung. Spenden und Subventionen vergrößern die Lohnstreuung.

\section{$17 \quad$ Ausblick}

Für einen möglichen Zusammenhang zwischen ehrenamtlichen Mitarbeiter/innen, Spenden und Subventionen auf Lohnhöhe und innerbetriebliche Lohnstreuung wurde in beiden Teilen der Dissertationen mehrere Vermutungen geäußert, wieso es diesen Zusammenhang gibt und wie Wirkungsrichtungen erklärt werden können. Die Ergebnisse zeigen, dass es die vermuteten Zusammenhänge zwischen den erwähnten Variablen gibt, offen bleibt jedoch, welche der verschiedenen, denkbaren Erklärungen zutrifft.

Grob zusammengefasst wirken sich ehrenamtliche Mitarbeiter/-innen negativ auf die Lohnhöhe und Lohnspreizung aus. Womöglich kann das mit der Arbeitsspendenhypothese erklärt werden. So können NPO unter Umständen in zwei Kategorien geteilt werden: Einerseits in eine Gruppe, in der ehrenamtliche Arbeit wichtig für die Tätigkeit der NPO ist, bzw. in eine Gruppe, wo die Missionsorientierung der NPO stark ausgeprägt ist. Diejenigen, die bezahlt werden, leisten womöglich aufgrund der Mission der NPO oder der Organisationskultur eine relativ hohe „Arbeitsspende“. Die Lohnstreuung bleibt in diesen Organisationen eher klein, weil vielleicht nur bestimmte Stellen im mittleren Lohnbereich besetzt sind. Andererseits könnte es NPO geben, die vorwiegend ,professionell" agieren und wo die Dienstleistungsbereitstellung besonderen Stellenwert hat. Hier sind die Löhne höher und auch die Lohnstreuung in derartigen Organisationen ist höher.

In Bezug auf Spenden und Subventionen kann grob zusammengefasst werden, dass höhere Zuwendungen - sowohl in Form von Spenden als auch in Form von öffentlichen Zuwendungen - zu höheren Löhnen und auch zu einer höheren innerbetrieblichen Lohnstreuung führen. Möglicherweise kann das Ergebnis mit höherer Zahlungsfähigkeit, d.h. mit Rent-Sharing, der NPO erklärt werden. 
Spenden und Subventionen erweitern das Budget der Organisation, was sich wiederum auf höhere Löhne oder durch mehr Anstellungen bezahlter Mitarbeiter auf die Lohnstreuung auswirkt. Allerdings profitieren, wie im ersten Teil der Dissertation zu sehen war, nicht alle Lohngruppen gleichermaßen von dieser Budgeterweiterung.

Noch einmal sei betont, dass das nur mögliche Erklärungen sind. Für eine genauere Untersuchung der Erklärungsgründe der Ergebnisse sind mehr Informationen vonnöten. Zum einen wäre für zukünftige Forschung interessant, ob es eine Rolle spielt, welche Aufgaben-verteilung bezahlte und unbezahlte Arbeitnehmer/-innen in NPO haben. Dazu wären detailliertere Angaben zur Art der Tätigkeit sowohl bei bezahlten als auch bei ehrenamtlichen Mitarbeiter/-innen notwendig.

Insgesamt erlauben die vorliegenden Daten aufgrund der Datenstruktur eine Untersuchung mit vielen Kontrollvariablen, die Qualität der Ergebnisse könnte jedoch verbessert werden, wenn weitere Variablen vorliegen würden, so z.B. genauere Angaben zur Ausbildung, zur Verweildauer im Unternehmen oder auch zu Fragen, wie z.B. einer Einschätzung der Arbeitnehmer/-innen über die Wichtigkeit der Mission der NPO. Mit diesen Variablen könnten genauere Aussagen darüber getroffen werden, welcher Erklärungsansatz für die Ergebnisse am zutreffendsten ist. Auch anhand von Paneldaten könnte nach Individualunterschieden kontrolliert werden. Teils fehlende Werte zu Informationen auf Organisationsebene schränken die Aussagekraft der Ergebnisse weiter ein. Zum tieferen Verständnis über die Zusammenhänge ist jedoch weitere Forschung auch in Form qualitativer Interviews - von großem Interesse.

In dieser Arbeit wurde angenommen, dass sich Spenden und Subventionen auf die Lohnhöhe und -streuung auswirken und dass nicht die umgekehrte Wirkungsrichtung gilt. Unter Umständen könnte sich, wie manchmal in der vorhandenen Literatur angenommen, z.B. die Lohnhöhe auch auf die Höhe der Spenden auswirken. Dies setzt jedoch voraus, dass die Löhne den Spender/-inne/n bekannt sind, was zurzeit für Österreich jedoch als nicht realistisch angenommen wird, da größtenteils Lohnverschwiegenheit herrscht. Sollte sich diese Annahme zukünftig ändern, müssten auch die vorgelegten Modelle dahingehend adaptiert werden und eine mögliche Endogenität dieser Variable berücksichtigt werden.

Diese Arbeit konnte dazu beitragen, erste Zusammenhänge eines weitgehend nicht erforschten Themas - der Determinanten der Lohnhöhe und der innerbetrieblichen Lohnstreuung im Nonprofit Sektor aufzuzeigen. Dazu wurde geklärt, welche Variablen die Lohnhöhe und Lohnstreuung beeinflussen, wobei besonderes Augenmerk auf nonprofit-spezifische Faktoren gelegt wurde. Hierfür wurden für Österreich einzigartige Arbeitnehmer-Arbeitgeber-Daten verwendet, wobei der gezeigte Zusammenhang auch für andere Länder Aussagekraft hat. 
Die Arbeit kann in verschiedene Richtungen weiter gedacht werden. So könnte die Untersuchung getrennt für eher dienstleistungsorientierte NPO und NPO, die vorwiegend eine Interessensvertretungsfunktion ausüben, durchgeführt werden. Ebenso wäre eine Trennung der Analyse der Lohnhöhe für Teilzeit- und Vollzeitkräfte womöglich sinnvoll, wenn sich Arbeitnehmer/-innen sich nicht zufällig in diese beiden Kategorien selbstselektieren, was sich wiederum auf die Löhne auswirken kann. Die erklärenden Variablen könnten unterschiedliche Auswirkungen auf die beiden Teilgruppen haben (vgl. Mocan, Tekin 2003).

Nicht betrachtet wurden in dieser Arbeit Lohnnebenleistungen, Arbeitsbedingungen und die Arbeitszufriedenheit. So kann nicht ausgeschlossen werden, dass beispielsweise Löhne in NPO mit ehrenamtlichen Arbeit zwar niedriger sind, diese niedrigeren Löhne jedoch durch Lohnnebenleistungen wie Firmenfahrzeuge oder durch einen höheren Urlaubsanspruch ,aufgewogen“ werden. Auch wissen wir anhand der Daten nur wenig über die Arbeitsbedingungen und die Arbeitszufriedenheit der Arbeitnehmer/-innen in den untersuchten österrreichischen NPO.

Zukünftige Forschung könnte auch an die vorwiegend amerikanische Tradition anschließen und Lohnhöhe und Lohnstreuung zwischen dem Nonprofit, gewinnorientierten und öffentlichen Sektor mit öster-reichischen Daten zu vergleichen. Hierzu wäre es sinnvoll, die Untersuchung auf einige Branchen zu beschränken, da in Österreich alle drei institutionellen Sektoren nur in wenigen Teilmärkten gleichzeitig agieren. 
Astrid Haider - 978-3-631-75377-4

Downloaded from PubFactory at 01/11/2019 05:43:53AM

via free access 


\section{8 Tabellen- und Abbildungsverzeichnis}

TABELLE 1: UNTERSCHIEDLICHE THEORIEN ZUR ERKLĀRUNG VON UNTERSCHIEDEN IN DER LOHNHOHE. .36

TABELLE 2: BRUTTOVERDIENSTE DER UNSELBSTÄNDIG ERWERBSTÄTIGEN 2005 NACH ABSCHNITTEN UND UNTERABSCHNITTEN DER ONACE 1995. 42

TABELLE 3: ÜBERBLICK ÜBER VORHANDENE EMPIRISCHE STUDIEN ................................................................59

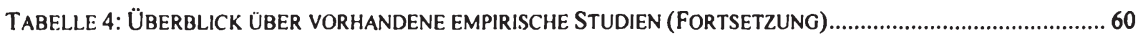

TABELLE 5: ÖKONOMETRISCHE IMPLEMENTATION DER VARIABLEN DER LOHNFUNKTION......................................68

TABELLE 6: ERWARTETE VORZEICHEN DER KOEFFIZIENTEN DER LOHNFUNKTION .............................................. 75

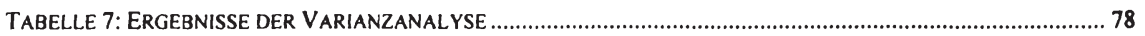

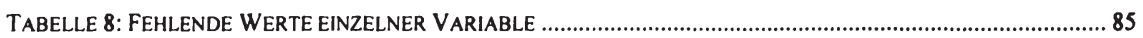

TABELLE 9: ERGEBNISVERGLEICH UNTER VERWENDUNG UNTERSCHIEDLICHE METHODEN IM UMGANG MIT

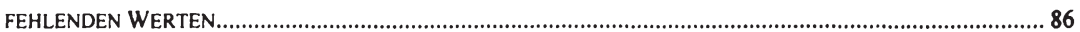

TABELLE 10: ANZAHL DER ORGANISATIONEN IM DATENSATZ NACH TÄTIGKEITSBEREICH ..................................88

TABELLE 11: ANZAHL DER ORGANISATIONEN IM DATENSATZ NACH BUNDESLAND.............................................8 89

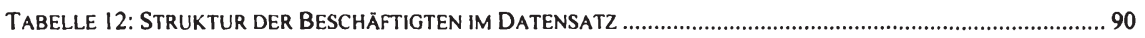

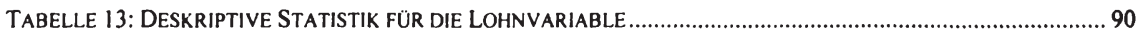

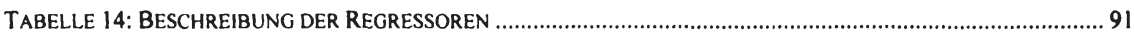

TABELLE 15: OLS-REGRESSIONEN, AUSGEWÄHLTE ERGEBNISSE ..................................................................... 93

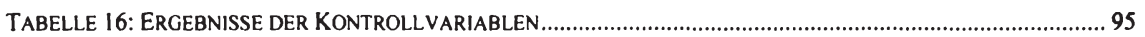

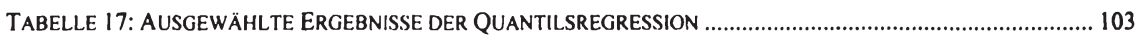

TABELLE 18: WEITERE ERGEBNISSE DER QUANTILSREGRESSION .................................................................... 105

TABELLE 19: WEITERE ERGEBNISSE DER QUANTILSREGRESSION (FORTS.) ......................................................... 106

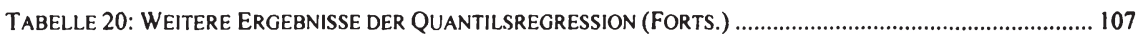

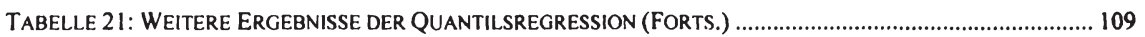

TABELLE 22: WEITERE ERGEBNISSE DER QUANTILSREGRESSION (FORTS.) ................................................... 110

TABELLE 23: ÜBERBLICK ÜBER RELEVANTE VORHANDENE LITERATUR IM BEREICH GEWINNORIENTIERTER

UNTERNEHMEN

TABELLE 24: ÜBERBLICK UBER RELEVANTE VORHANDENE LITERATUR IM BEREICH DER NPO ODER NONPROFITNAHEN ORGANISATIONEN . 149

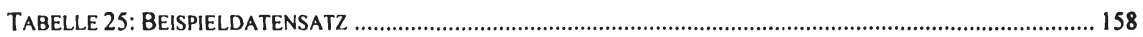

TABELLE 26: STANDARDABWEICHUNG UND VARIANZ IM BEISPIELDATENSATZ .............................................. 158

TABELLE 27: BESCHREIBUNG DER REGRESSOREN UND ERWARTETE VORZEICHEN ............................................ 161

TABELLE 28: HĀUFIGKEIT DER ORGANISATIONEN NACH ANZAHL DER MITARBEITER/-INNEN ............................ 165

TABELLE 29: DESKRIPTIVE STATISTIK DER VERWENDETEN VARIABLEN .......................................................... 166

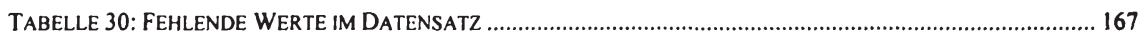

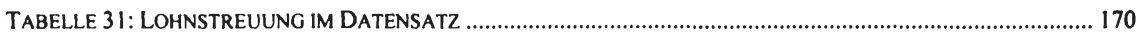

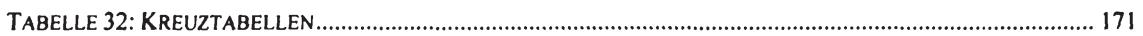

TABELLE 33: SCHÄTZUNG DER LOHNSTREUUNG, UMFASSENDES MODELL („LANG-VARIANTE“), VERSCHIEDENE ABHÄNGIGE VARIABLE. 173

TABELLE 34: SCHĀTZUNG DER LOHNSTREUUNG (,KURZVARIANTE“), VERSCHIEDENE ABHÃNGIGE VARIABLE .. 176 TABELLE 35: ERGEBNISSE DER SCHĀTZUNG AUF DIE LOHNSTREUUNG BEI VERWENDUNG UNTERSCHIEDLICHER METHODEN ZUR KORREKTUR FEHLENDER WERTE. 
ABBILDUNG 1: ÜBERSICHT ÜBER RELEVANTE ERKLÄRUNGSANSÄTZE .35

ABBILDUNG 2: KORRELATION GESCHLECHT UND LOHN .160 
Abowd, John M.; Kramarz, Francis (2000): "Inter-industry and Firm-size Wage Differentials in the United States", in: Cornell University working paper.

Akerlof, George A. (1970): "The Market for "Lemons": Quality Uncertainty and the Market Mechanism", in: Quarterly Journal of Economics, 84 (3). 488500 .

Akerlof, George A. (1982): "Labor Contracts as Partial Gift Exchange", in: The Quarterly Journal of Economics, 97 (4). 543-569.

Akerlof, George A.; Yellen, Janet L. (1990): "The Fair Wage-Effort Hypothesis and Unemployment", in: The Quarterly Journal of Economics, 105 (2). 255-283.

Allison, Paul David (2002): Missing Data. Thousand Oaks: Sage.

Andini, Corrado (2007): "Returns to education and wage equations: a dynamic approach", in: Applied Economics Letters, 14 (8). 577.

Backhaus, Klaus; Erichson, Bernd; Plinke, Wulff; Weiber, Rolf (2000): Multivariate Analysemethoden. 9. Auflage. Berlin, Heidelberg, New York: Springer Verlag.

Badelt, Christoph (2002): Zielsetzungen und Inhalte des "Handbuchs der Nonprofit Organisationen". in: Badelt, Christoph (Hrsg.): Handbuch der Nonprofit Organisatonen. Stuttgart: Schäffer-Poeschel Verlag. 3-18.

Badelt, Christoph; Hollerweger, Eva (2007): Ehrenamtliche Arbeit im Nonprofit Sektor, in: Badelt, Christoph; Meyer, Michael; Simsa, Ruth (Hrsg.): Handbuch der Nonprofit Organisationen. Strukturen und Management. Stuttgart: Schäffer-Poeschel. 503-531.

Badelt, Christoph; Meyer, Michael; Simsa, Ruth (2007a): Ausblick: Entwicklungsperspektiven des Nonprofit Sektors, in: Badelt, Christoph; Meyer, Michael; Simsa, Ruth (Hrsg.): Handbuch der Nonprofit Organisationen. Strukturen und Management. Stuttgart: Schäffer-Poeschel.

Badelt, Christoph; Meyer, Michael; Simsa, Ruth (2007b): Die Wiener Schule der NPO-Forschung, in: Badelt, Christoph; Meyer, Michael; Simsa, Ruth (Hrsg.): Handbuch der Nonprofit Organisatonen. Strukturen und Management. Stuttgart: Schäffer-Poeschel. 3-16.

Ballou, Jeffrey P.; Weisbrod, Burton A. (2003): "Managerial Rewards and the Behavior of For-Profit, Governmental and Nonprofit Organizations: Evidence from the Hospital Industry." in: Journal of Public Economics, 87 (9-10). 1895-1920.

Barsky, Carl B.; Personick, Martin E. (1981): "Measuring wage dispersion: pay ranges reflect industry traits", in: Monthly Labor Review, 104 (4). 35 41.

Beaumont, P.B.; Harris, R.I.D. (2003): "Internal Wage Structures and Organizational Performance", in: British Journal of Industrial Relations, 41 (1). 53-70. 
Bechtel, Stephan; Heinbach, Wolf Dieter; Strotmann, Harald (2005): Tarifbindung, betriebliche Lohnhöhe und Lohnstreuung im Produzierenden Gewerbe Baden-Württembergs, in: Forschungsdatenzentrum der Statistischen Landesämter (Hrsg.): Amtliche Mikrodaten für die Sozial- und Wirtschaftswissenschaften, Beiträge zu den Nutzerkonferenzen des FDZ der Statistischen Landesämter 2005. Düsseldorf: Statistische Landesämter. $159-178$.

Becker, Gary Stanley (1975): Human capital: a theoretical and empirical analysis, with special reference to education. 2. New York.

Becker, Silke (2000): Lohnstrukturen. Eine betriebswirtschaftliche Untersuchung. München, Mering: Rainer Hampp Verlag.

Bellmann, Lutz; Bender, Stefan; Kölling, Arnd (2002): Der Linked EmployerEmployee-Datensatz aus IAB-Betriebspanel und Beschäftigtenstatistik der Bundesanstalt für Arbeit (LIAB), in: Kleinhenz, Gerhard (Hrsg.): IAB-Kompendium Arbeitsmarkt- und Berufsforschung. 21-30.

Ben-Ner, Avner (1986): Nonprofit Organizations: Why do They Exist in Market Economics? in: Rose-Ackerman, Susan (Hrsg.): The Economics of Nonprofit Institutions. Studies in Structure and Policy. New York, Oxford: Oxford University Press. 94-113.

Benz, Matthias (2005): "Not for the Profit, but for the Satisfaction? - Evidence on Worker Well-Being in Non-Profit Firms", in: Kyklos, 58 (2). 155 177.

Blackburn, McKinley L. (2007): "Estimating wage differentials without logarithms", in: Labour Economics, 14 (1). 73-98.

Blaise, Melly (2005): "Public-private sector wage differentials in Germany: Evidence from quantile regression", in: Empirical Economics, 30 (2). 505-520.

Blümle, Gerold (1975): Theorie der Einkommensverteilung. Berlin, Heidelberg, New York: Springer-Verlag.

Bönisch, Markus (2008): "Kollektivvertragliche Abdeckung in Österreich", in: Statistische Nachrichten, 3/2008. 207-211.

Borjas, George J. (2005): Labor Economics. 3rd. Boston: McGraw-Hill Irwin. Borjas, George J.; Frech, H. E., III; Ginsburg, Paul B. (1983): "Property Rights and Wages: The Case of Nursing Homes", in: The Journal of Human Resources, 18 (2). 231-247.

Brandl, Julia; Güttel, Wolfgang H.; Konlechner, Stefan; Beisheim, Margret; Eckardstein, Dudo von; Elsik, Wolfgang (2006): "Entwicklungsdynamik von Vergütungssystemen in Nonprofit-Organisationen", in: Zeitschrift für Personalforschung, 20 (4). 356-374.

Bronars, Stephen G.; Famulari, Melissa (1997): "Wage, Tenure, and Wage Growth Variation Within and Across Establishments", in: Journal of Labor Economics, 15 (2). 285-317. 
Brown, Eleanor; Slivinski, Al (2006): Nonprofit Organizations and the Market, in: Powell, Walter W.; Steinberg, Richard (Hrsg.): The Nonprofit Sector. A Research Handbook. New Haven, London: Yale University Press. 140158.

Buchinger, Clemens; Trukeschitz, Birgit; Schneider, Ulrike (2008): "Leistungsbezogene öffentliche Finanzierung von Nonprofit Organisationen im österreichischen Sozialwesen. Formen und Bedeutung von Leistungsverträgen sowie Eigenschaften sozialer NPOs mit Leistungsverträgen", in: Forschungsbericht des Instituts für Sozialpolitik, 01/2008.

Buchinsky, Moshe (1998): "Recent Advances in Quantile Regression Models", in: The Journal of Human Resources, 33 (1). 88-126.

Burke, L.A.; Witt, L.A. (2004): "Personality and High-maintenance Employee Behavior", in: Journal of Business and Psychology, 18 (3). 349-363.

Cain, Glen G. (1986): The Economic Analysis of Labor Market Discrimination: A Survey, in: Ashenfelter, Orley C.; Layard, Richard (Hrsg.): Handbook of Labor Economics. Amsterdam, New York, Oxford, Tokyo: Elsevier. 1. 693-785.

Canal Dominguez, Juan Francisco; Rodriguez Gutierrez, Cesar (2004): "Collective Bargaining and Within-firm Wage Dispersion in Spain", in: British Journal of Industrial Relations, 42 (3). 481-506.

Cardoso, Ana Rute (1997): "Workers or Employers: Who Is Shaping Wage Inequality?" in: Oxford Bulletin of Economics and Statistics, 59 (4). 523 547.

Charness, Gary; Kuhn, Peter (2007): "Does Pay Inequality Affect Worker Effort? Experimental Evidence", in: Journal of Labor Economics, 25 (4). 693-723.

Choi, Kang-Shik; Jeong, Jinook (2007): "Does unmeasured ability explain the wage premium associated with technological change? Quantile regression analysis", in: Applied Economics, 39 (9). 1163 - 1171.

Cowell, Frank A. (1995): Measuring Inequality. Second. London, New York: Prentice Hall/Harvester Wheatsheaf.

Cowherd, Douglas M.; Levine, David I. (1992): "Product Quality and Pay Equity Between Lower-level Employees and Top Management: An Investigation of Distributive Justice Theory", in: Administrative Science Quarterly, 37 (2). 302-320.

Criscuolo, Chiara (2000): "Employer Size-Wage Effect: A Critical Review and an Econometric Analysis", in: Working Paper: Università degli Studi di Siena. Dipartimento di Economia Politica, 277.

Davis, Steven J.; Haltiwanger, John C. (1991): "Wage Dispersion between and within U.S. Manufacturing Plants, 1963-1986", in: Brookings Papers on Economic Activity. Microeconomics., 1991. 115-200.

Davis, Steven J.; Haltiwanger, John C. (1995): "Employer Size and the Wage Structure in U.S. Manufacturing", in: NBER Working Paper Series, 5393. 
Dell'Aringa, Carlo; Lucifora, Claudio (1994): "Wage Dispersion and Unionism: Do Unions Protect Low Pay?" in: International Journal of Manpower, 15 (2/3). 150-169.

Dell'Aringa, Carlo; Pagani, Laura (2007): "Collective Bargaining and Wage Dispersion in Europe", in: British Journal of Industrial Relations, 45 (1). 29-54.

Depken II, Craig A. (2000): "Wage disparity and team productivity: evidence from major league baseball", in: Economics Letters, 67. 87-92.

Devaro, Jed; Brookshire, Dana (2007): "Promotions and Inventives in Nonprofit and For-Profit Oranizations", in: Industrial and Labor Relations Review, 60 (3). 311-339.

Dickens, William T.; Katz, Lawrence F.; Dickens, William T. and Katz, Lawrence F., "Interindustry Wage Differences and Industry Characteristics" (August 1987). NBER Working Paper No. W2014. (1987): "Interindustry Wage Differences and Industry Characteristics ", in: NBER Working Paper, No.W2014.

Dimmel, Nikolaus (2005): Perspektiven der Sozialwirtschaft 2005-2015. Vergaberecht - Leistungsverträge - Sozialplanung. Wien: Lit Verlag.

Eckardstein, Dudo von (2007): Personalmanagement in NPOs, in: Badelt, Christoph; Meyer, Michael; Simsa, Ruth (Hrsg.): Handbuch der Nonprofit Organisationen. Strukturen und Management. Stuttgart: SchäfferPoeschel Verlag. 273-298.

Efron, Bradley; Tibshirani, Robert J. (1993): An introduction to the bootstrap. New York: LinkChapman\&Hall.

Emanuele, Rosemarie (1996): "Is there a (downward sloping) demand curve for volunteer labour?" in: Annals of Public and Cooperative Economics, 67 (2). 193-208.

Farber, Henry S. (1986): The Analysis of Union Behavior, in: Ashenfelter, Orley C.; Layard, Richard (Hrsg.): Handbook of Labor Economics. Amsterdam, New York, Oxford, Tokyo: Elsevier. 2. 1039-1089.

Feldstein, Martin S. (1971): The Rising Cost of Hospital Care. Washington D.C.: Information Resources Press.

Fitzenberger, Bernd; Kohn, Karsten (2005): "Gleicher Lohn für gleiche Arbeit? Zum Zusammenhang zwischen Gewerkschaftsmitgliedschaft und Lohnstruktur in Westdeutschland 1985-1997", in: ZEW Discussion Paper, 06006.

Frank, Robert (1984): "Are Workers Paid Their Marginal Product?" in: American Economic Review, 74 (5). 549-571.

Frank, Robert (1996): "What Price the High Moral Ground", in: Southern Economic Journal, 63 (1). 1 - 17.

Franz, Wolfgang (2006): Arbeitsmarktökonomik. 6. Auflage. Berlin, Heidelberg, New York: Springer. 
Freeman, Richard B. (1980): "Unionism and the Dispersion of Wages", in: Industrial and Labor Relations Review, 34 (1). 3-23.

Freeman, Richard B. (1982): "Union Wage Practices and Wage Dispersion within Establishments", in: Industrial and Labor Relations Review, 36 (1). 3-21.

Frumkin, Peter; Keating, Elizabeth (2001): "The Price of Doing Good: Executive Compensation in Nonprofit Organizations", in: Hauser Center Working Paper Series. Harvard University, 8.

Gabler Wirtschafts Lexikon (2004): Gabler Wirtschafts Lexikon. 16. Auflage. Wiesbaden: Betriebswirtschaftlicher Verlag Dr. Th. Gabler / GWV Fachverlage GmbH.

Gaskin, Katherine; Smith, Justin Davis (1995): A New Civic Europe? A study of the extent and role of volunteering. Second Edition. London: The Volunteer Centre UK.

Geigant, Friedrich; Haslinger, Franz; Sobotka, Dieter; Westphal, Horst M. (2000): Lexikon Volkswirtschaft. 7. überarbeitete Auflage. Landsberg am Lech: Verlag Moderne Industrie.

Gerlach, Knut; Stephan, Gesine (2005): "Wage Distribution by Wage-Setting Regime", in: IAB Discussion Paper, 9/2005.

Gerlach, Knut; Stephan, Gesine (2006a): "Bargaining Regimes and Wage Dispersion", in: Jahrbücher für Nationalökonomie und Statistik, 226 (6). 629-645.

Gerlach, Knut; Stephan, Gesine (2006b): "Pay Policies of Firms and Collective Wage Contracts - An Uneasy Partnership?" in: Industrial Relations, 45 (1). $47-67$.

Goddeeris, John H. (1988): "Compensating Differentials and Self-Selection: An Application to Lawyers", in: The Journal of Political Economy, 96 (2). 411-428.

Gosling, Amanda; Machin, Stephen (1995): "Trade Unions and the Dispersion of Earnings in British Establishments, 1980-1990", in: Oxford Bulletin of Economics and Statistics, 57 (2). 167-184.

Gould, William W. (1992): "sg11.1: Quantile regression with bootstrapped errors." in: Stata Technical Bulletin, 9. 19-21.

Gould, William W. (1997): "sg70: Interquartile and simultaneous-quantile regression", in: Stata Technical Bulletin, 38. 14-22.

Gray, Samuel R.; Benson, Philip G. (2003): "Determinants of Executive Compensation in Small Business Development Centers", in: Nonprofit Management and Leadership, 13 (3). 213-227.

Greene, William H. (2003): Econometric Analysis. International Edition. 5th edition. Upper Saddle River, NJ: Pearson Prentice Hall.

Grepperud, Sverre; Pedersen, Pal Andreas (2006): "Crowding Effects and Work Ethics", in: Labour, 20 (1). 125-138. 
Griliches, Zvi (1986): Economic Data Issues, in: Griliches, Zvi; Intriligator, Michael D. (Hrsg.): Handbook of Econometrics. Amsterdam, New York, Oxford, Tokyo: Elsevier Science Publishers. 3. 1465-1514.

Groshen, Erica L. (1991a): "Five Reasons Why Wages Vary Among Employers", in: Industrial Relations, 30 (3). 350-381.

Groshen, Erica L. (1991b): "Sources of Intra-industry Wage Dispersion: How Much Do Employer Matter?" in: Quarterly Journal of Economics, 106 (3). 869-884.

Gunderson, Morley (2006): "Viewpoint: Male-female wage differentials: how can that be?" in: Canadian Journal of Economics, 39 (1). 1-21.

Gusenleitner, Markus; Winter-Ebmer, Rudolf; Zweimüller, Josef (1998): "The Distribution of Earnings in Austria, 1972 - 1991", in: Allgemeines Statistisches Archiv, 82. 275-290.

Hackl, Peter; Glatzer, Ernst (2000): Ökonometrie. Vortragsskript. 6. Auflage. Wien: Facultas.

Haider, Astrid; Leisch, Robert; Schneider, Ulrike; Stöger, Klaus (2008): "Neue Datengrundlagen für den Non-Profit Bereich", in: Statistische Nachrichten, August 2008. 754-762.

Hallock, Kevin F. (2002): "Managerial Pay and Governance in American Nonprofits", in: Industrial Relations: A Journal of Economy \& Society, 41 (3). 377-406.

Haltiwanger, John C.; Davis, Steven J. (1996): "Employer Size and the Wage Structure in U.S. Manufacturing", in: Annales d'Economie et de Statistique, 41/42 (4). 324-367.

Haltiwanger, John C.; Lane, Julia I.; Spletzer, James R.; Theeuwes, Jules J.M.; Troske, Kenneth R. (1999): The Creation and Analysis of EmployerEmployee Matched Data. Amsterdam: Elsevier Science B.V.

Hamermesh, Daniel S. (1999): "LEEping into the future of labor economics: the research potential of linking employer and employee data", in: Labour Economics, 6 (1). 25-41.

Handy, Femida; Mook, Laurie; Quarter, Jack (2008): "The Interchangeability of Paid Staff and Volunteers in Nonprofit Organizations", in: Nonprofit and Voluntary Sector Quarterly, 37 (1). 76-92.

Hansmann, Henry B. (1980): "The Role of Nonprofit Enterprise", in: The Yale Law Journal, 89 (5). 835.

Hartog, Joop; Pereira, Pedro T.; Vieira, José A.C. (2000): "Inter-industry Wage Dispersion in Portugal", in: Empirica, 27 (4). 353-365.

Heitzmann, Karin (2001): Dimensionen, Strukturen und Bedeutung des Nonprofit Sektors. Eine theoretisch-konzeptionelle und empirische Analyse für Österreich. Wien: Facultas Copy Store.

Hesse, Nils; Rivas, Maria Fernanda (2007): "Managerial Compensation in a Two-Level Gift-Exchange Experiment", in: Documentos de Trabajo (working papers), Department of Economics - dECON, 23/07. 
Heyman, Fredrik (2002): Pay Inequality and Firm Performance: Evidence from Matched Employer-Employee Data: FIEF Working Paper Series 2002.

Heyman, Fredrik (2005): "Pay inequality and firm performance: evidence from matched employer-employee data", in: Applied Economics, 37 (11). 1313-1327.

Hibbs Jr., Douglas A.; Locking, Hakan (2000): "Wage Dispersion and Productive Efficiency: Evidence for Sweden", in: Journal of Labor Economics, 18 (4). 755-782.

Hiller, Tobias (2006): "Die Humankapitaltheorie. Ein theoretischer Erklärungsansatz für Lohndifferenziale", in: WiSt, 5. 285-288.

Hirsch, Barry T. (1982): "The Interindustry Stucture of Unionism, Earnings, and Earnings Dispersion", in: Industrial and Labor Relations Review, 36 (1). 22-39.

Holtmann, Alphonse G.; Idson, Todd L. (1993): "Wage Determination of Registered Nurses in Proprietary and Nonprofit Nursing Homes", in: The Journal of Human Resources, 28 (1). 55-79.

Horowitz, Joel L. (2001): The Bootstrap, in: Heckman, James J.; Leamer, Edward (Hrsg.): Handbook of Econometrics. Amsterdam, London, New York et al.: Elsevier Science. 5. 3160-3228.

Horton, Nicholas J.; Kleinman, Ken P. (2007): "Much Ado About Nothing: A Comparison of Missing Data Methods and Software to Fit Incomplete Data Regression Models", in: The American Statistician, 61 (1). 79-90.

Hübler, Olaf (2003): "Geschlechtsspezifische Lohnunterschiede", in: Mitteilungen aus der Arbeitsmarkt- und Berufsforschung (MittAB), 36. 539559.

Ito, Takatoshi; Domain, Dale (1987): "A Musical Note on the Efficiency Wage Hypothesis - Programmings, Wages and Budgets of American Symphony Orchestras." in: Economics Letters, 25. 95 - 99.

James, Estelle Dinerstein; Rose-Ackerman, Susan (1986): "The nonprofit enterprise in market economics", in: Chur: Harwood, 7 (9). 102.

James Jr., Harvey S (2005): "Why did you do that? An economic examination of the effect of extrinsic compensation on intrinsic motivation and performance", in: Journal of Economic Psychology, 26 (4). 549-566.

James, Robert G.; Morlock, Mark J. (1987): "The determinants of intraoccupational wage dispersion", in: Applied Economics, 19 (7). 969-981.

Jirjahn, Uwe; Kraft, Kornelius (2007): "Intra-firm Wage Dispersion and Firm Performance - Is There a Uniform Relationship?" in: Kyklos, 60 (2). 231 253.

John, Oliver P.; Srivastava, Sanjay (1999): Big Five Trait Taxonomy, in: Pervin, Lawrence A.; John, Oliver P. (Hrsg.): Handbook of Personality: Theory and Reserach. New York: Guilford. 102-138. 
Jones, Michael P. (1996): "Indicator and Stratification Methods for Missing Explanatory Variables in Multiple Linear Regression", in: Journal of the American Statistical Association, 91 (433). 222-230.

Kahn, Lawrence M. (1998): "Collective bargaining and the interindustry wage structure: International evidence", in: Economica, 65 (260). 507-534.

Kahn, Lawrence M.; Curme, Michael (1987): "Unions and Nonunion Wage Dispersion", in: The Review of Economics and Statistics, 69 (4). 600607.

Keller, Robert T. (2007): "Predicting Job Performance from Individual Characteristics among R\&D Engineers", in: The Business Review, 8 (1). 1218.

Klodt, Thomas (2000): Produktmärkte, Rent Sharing und Lohnhöhe. Eine ökonometrische Analyse mit Betriebspaneldaten. Frankfurt/Main: Campus Verlag GmbH.

Koenker, Roger (2005): Quantile Regression. Cambridge, New York et al.: Cambridge University Press.

Koenker, Roger; Bassett, Gilbert Jr. (1978): "Regression Quantiles", in: Econometrica, 46 (1). 33-50.

Kornai, Janos (1986): "The soft budget constraint", in: Kyklos, 39 (1). 3-30.

Kornai, Janos; Maskin, Eric; Roland, Gerard (2003): "Understanding the soft budget constraint", in: Journal of Economic Literature, 41 (4). 10951136.

Kremer, Michael; Maskin, Eric (1996): "Wage Inequality and Segregation by Skill", in: NBER Working Paper Series, 5718.

Lakdawalla, Darius; Philipson, Tomas (1998): "Nonprofit Production and Competition", in: NBER Working Paper, No. 6377.

Lallemand, Thierry; Plasman, Robert; Rycx, Francois (2004): "Intra-Firm Wage Dispersion and Firm Performance: Evidence from Linked EmployerEmployee Data", in: Kyklos, 57 (4). 533-558.

Lallemand, Thierry; Plasman, Robert; Rycx, Francois (2005): "Why do larger firms pay higher wages? Evidence from matched worker-firm data." in: International Journal of Manpower, 26 (7/8). 705.

Lallemand, Thierry; Plasman, Robert; Rycx, Francois (2007a): "The establishment-size wage premium: evidence from European countries", in: Empirica, 34 (5). 427-451.

Lallemand, Thierry; Plasman, Robert; Rycx, Francois (2007b): Wage Structure and Firm Producitivity in Belgium, in: Lazear, Edward P.; Shaw, Kathryn L. (Hrsg.): An International Comparison of the Structure of Wages: University of Chicago Press, NBER.

Lallemand, Thierry; Rycx, Francois (2006): "Establishment Size and the Dispersion of Wages: Evidence from European Countries", in: Applied Economics Quarterly, 52 (4). 309-336. 
Landmann, Oliver; Jerger, Jürgen (1999): Beschäftigungstheorie. Berlin, Heidelberg, New York: Springer.

Lanfranchi, Joseph; Narcy, Mathieu (2008): "Différences de satisfaction dans l'emploi entre secteurs à but lucratif et à but non lucratif: Le role joué par les caractéristiques d'emploi", in: Annals of Public and Cooperative Economics, 79 (2). 323-368.

Lazear, Edward P. (1991): "Labor Economics and the Psychology of Organizations", in: Journal of Economic Perspectives, 5 (2). 89-110.

Lazear, Edward P.; Rosen, Sherwin (1981): "Rank-Order Tournaments as Optimum Labor Contracts", in: The Journal of Political Economy, 89 (5). 841-864.

Lazear, Edward P.; Shaw, Kathryn L. (2007): Wage Structure, Raises and Mobility: International Comparisons of the Structure of Wages Within and Across Firms, in: Lazear, Edward P.; Shaw, Kathryn L. (Hrsg.): An International Comparison of the Structure of Wages: University of Chicago Press, NBER.

Leete, Laura (2000): "Wage equity and employee motivation in nonprofit and for-profit organizations", in: Journal of Economic Behavior and Organization, 43. 423-446.

Leete, Laura (2001): "Whither the Nonprofit Wage Differential? Estimates from the 1990 Census", in: Journal of Labor Economics, 19 (1). $136-169$.

Leete, Laura (2006): Work in the Nonprofit Sector, in: Powell, Walter W.; Steinberg, Richard (Hrsg.): The Nonprofit Sector - A Research Handbook. New Haven, London: Yale University Press. 159 - 179.

Lemieux, Thomas (2002): "Decomposing Changes in Wage Distributions: A Unified Approach", in: The Canadian Journal of Economics, 35 (4). 646688.

Lemieux, Thomas (2006): The "Mincer Equation" Thirty Years after Schooling, Experience, and Earnings, in: Grossbard-Shechtman, Shoshana (Hrsg.): Jacob Mincer, A Pioneer of Modern Labor Economics. New York: Springer Science+Business Media. 127-145.

Levine, David I. (1991): "Cohesiveness, Productivity, and Wage Dispersion", in: Journal of Economic Behavior and Organization, 15. 237-255.

Lewis, H. Gregg (1986): Union Relative Wage Effects, in: Ashenfelter, Orley C.; Layard, Richard (Hrsg.): Handbook of Labor Economics. Amsterdam, New York, Oxford, Tokyo: Elsevier. 2. 1139-1181.

Lexikon der Geographie (2001). Heidelberg: Spektrum Akademischer Verlag.

Littich, Edith (2007): Finanzierung von NPOs, in: Badelt, Christoph; Meyer, Michael; Simsa, Ruth (Hrsg.): Handbuch der Nonprofit Organisationen. Stuttgart: Schäffer-Poeschel. 322-339.

Little, Roderick J. A.; Rubin, Donald B. (2002): Statistical analysis with missing data. 2nd. New York: Wiley. 
Machado, José A.F.; Mata, José (2005): "Counterfactual Decomposition of Changes in Wage Distributions Using Quantile Regression", in: Journal of Applied Econometrics, 20 (4). 445-465.

Machin, Stephen; Manning, Alan (2004): "A Test of Competitive Labor Market Theory: The Wage Structure Among Care Assistants in the South of England", in: Industrial and Labor Relations Review, 57 (3). 371-385.

Manning, Alan (1994): "Labour Markets with Company Wage Policies", in: CEP Discussion Paper, 0214.

Medoff, James; Brown, Charles (1989): "The Employer Size-Wage Effect", in: Journal of Political Economy, 97 (5). 1027-1059.

Melly, Blaise (2005): "Public-private sector wage differentials in Germany: Evidence from quantile regression", in: Empirical Economics, 30 (2). 505-520.

Menchik, Paul L.; Weisbrod, Burton A. (1987): "Volunteer Labor Supply", in: Journal of Public Economics, 32 (2). 159-183.

Meszaros, Gerhard (2007): NPO-Gehälter: Generell niedriger. Die Presse. 8.7.2007. K5.

Metcalf, David; Hansen, Kirstine; Charlwood, Andy (2000): "Unions and the Sword of Justice: Unions and Pay Systems, Pay Inequality, Pay Discrimination and Low Pay." in: Center for Economic Performance Discussion Paper, 452.

Meyer, Michael (2007): Von der öffentlichen Bürokratie zur technokratischen NPO? Über die Effekte leistungsvertraglicher Finanzierungsformen auf die Organisationsstrukturen von NPO, in: Schneider, Ulrike; Trukeschitz, Birgit (Hrsg.): Quasi-Märkte und Qualität. Die Qualität arbeitsmarktpolitischer und sozialer Dienstleistungen im Kontext öffentlicher Beschaffungspolitik. Baden-Baden: Nomos. 85-102.

Meyers Großes Taschenlexikon in 24 Bänden.

Mincer, Jacob (1958): "Investment in Human Capital and Personal Income Distribution", in: Journal of Political Economy, 66 (4). 281-302.

Mirvis, Philip H.; Hackett, Edward J. (1983): "Work and work force characteristics in the nonprofit sector", in: Monthly Labor Review, 116 (4). 312.

Mocan, H. Naci; Tekin, Erdal (2003): "An Analysis of Employer-Employee Matched Data of Child Care Workers", in: The Review of Economics and Statistics, 85 (1). 38-50.

Mocan, H. Naci; Viola, Deborah (1997): "The Determinants of Child Care Workers' Wages and Compensation: Sectoral Difference, Human Capital, Race, Insiders and Outsiders", in: NBER Working Paper Series, 6328.

Mortensen, Dale T.; Pissarides, Christopher A. (1999): New Developments in Models of Search in the Labor Markets, in: Ashenfelter, Orley C.; Card, David (Hrsg.): Handbook of Labor Economics. Amsterdam: Elsevier Science B.V. 3B. 2567-2627. 
Mosca, Michele; Musella, Marco; Pastore, Francesco (2007): "Relational goods, monitoring and non-pecuniary compensations in the nonprofit sector: The case of the Italian social services", in: Annals of Public and Cooperative Economics, 78 (1). 57-86.

Mueller, Gerrit; Plug, Erik (2006): "Estimating the effect of personality on male and female earnings", in: Industrial and Labor Relations Review, 60 (1). 3-22.

Netting, F. Ellen; O'Connor, Mary Katherine; Thomas, M. Lori; Yancey, Gaynor (2005): "Mixing and Phasing of Roles Among Volunteers, Staff, and Participants in Faith-Based Programs", in: Nonprofit and Voluntary Sector Quarterly, 34 (2). 179-205.

Neumayr, Michaela; Schneider, Ulrike; Meyer, Michael; Haider, Astrid (2007): The Non-profit Sector in Austria - An economic, legal and political appraisal. Vienna: Vienna University for Economics and Business Administration.

Noguchi, Haruko; Shimizutani, Satoshi (2007): "Nonprofit/for-profit status and earning differentials in the Japanese at-home elderly care industry: Evidence from micro-level data on home helpers and staff nurses", in: Journal of the Japanese and International Economies, 21 (1). 106-120.

Nordström Skans, Oskar; Edin, Per-Anders; Holmlund, Bertil (2007): "Wage dispersion between and within plants: Sweden 1985-2000", in: NBER Working Paper, 13021.

Nyhus, Ellen K.; Pons, Empar (2006): "The effects of personality on earnings", in: Journal of Economic Psychology, 26 (3).

OECD (1997): Employment Outlook. Paris: OECD.

OECD (2004): Employment Outlook. Paris: OECD.

Oi, Walter Y.; Idson, Todd L. (1999): Firm Size and Wages, in: Ashenfelter, Orley C.; Card, David (Hrsg.): Handbook of Labor Economics. Amsterdam et al.: Elsevier. 3B. 2165-2212.

Oster, Sharon (1998): "Executive Compensation in the Nonprofit Sector", in: Nonprofit Management and Leadership, 8. 207 - 221.

Ostermeier, Christian (2002): Klassifikation und Systematisierung des Nonprofit-Sektors. Systematische Darstellung und Analyse bestehender Typologie- und Klassifikationsansätze unter Berücksichtigung nationaler und internationaler Anwendungsmöglichkeiten. Diplomarbeit. Wirtschaftsuniversität Wien.

Österreichisches Institut für Spendenwesen (2007): Spendenbericht 2007. Wien: Österreichisches Institut für Spendenwesen.

Pfeffer, Jeffrey; Davis-Blake, Alison (1990): "Determinants of Salary Dispersion in Organizations", in: Industrial Relations, 29 (1). 38-57.

Pfeffer, Jeffrey; Langton, Nancy (1993): "The Effect of Wage Dispersion on Satisfaction, and Working Collaboratively: Evidence from College and 
University Faculty", in: Administrative Science Quarterly, 38 (3). 382407.

Piekkola, Hannu; Kauhanen, Antti (2003): "Rent sharing as firm-level pay", in: International Journal of Manpower, 24 (4). 426-451.

Pindyck, Robert S.; Rubinfeld, Daniel L. (2005): Mikroökonomie. 6. Auflage. München: Pearson Studium.

Preston, Anne E. (1988): "The Effects of Property Rights on Labor Costs of Nonprofit Firms: An Application to the Day Care Industry." in: Journal of Industrial Economics, 36. 337 - 350.

Preston, Anne E. (1989): "The Nonprofit Worker in a For-Profit World", in: Journal of Labor Economics, 7 (4). 438-463.

Preston, Anne E. (1990): "Women in the White-Collar Nonprofit Sector: The Best Option or the Only Option?" in: Review of Ecnonomics and Statistics, 72 (4). 560-568.

Rodriguez Gutierrez, Cesar (2001): "Wage dispersion within firms and collective bargaining in Spain", in: Economics Letters, 72. 381-386.

Roomkin, Myron J.; Weisbrod, Burton A. (1999): "Managerial Compensation and Incentives in For-Profit and Nonprofit Hospitals", in: Journal of Law Economics \& Organization, 15 (3). 750-781.

Rose-Ackerman, Susan (1996): "Altruism, Nonprofits, and Economic Theory", in: Journal of Economic Literature, 34. 701 - 728.

Rose-Ackermann, Susan (Ed.) (1986): The Economics of Nonprofit Institutions. New York, Oxford: Oxford University Press.

Ross, Helmut (1981): Theorie der internen Lohnstruktur: Ein kritischer Vergleich der Aussagekraft vorherrschender Arbeitsmarkttheorien hinsichtlich der Inflexibilität der internen Lohnstruktur. Frankfurt am Main: Verlag Peter Lang GmbH.

Rotolo, Thomas; Wilson, John (2006): "Employment Sector and Volunteering: The Contribution of Nonprofit and Public Sector Workers to the Volunteer Labor Force." in: The Sociological Quarterly, 47. 21-40.

Ruhm, Christopher J.; Borkoski, Carey (2003): "Compensation in the Nonprofit Sector", in: Journal of Human Resources, 28 (4). 992 - 1021.

Runggaldier, Ulrich; Drs, Monika (2007): Arbeits- und sozialrechtliche Rahmenbedingungen beim Einsatz von MitarbeiterInnen in NPOs, in: Badelt, Christoph; Meyer, Michael; Simsa, Ruth (Hrsg.): Handbuch der Nonprofit Organisationen. Strukturen und Management. Stuttgart: Schäffer-Poeschel Verlag. 299-321.

Salamon, Lester M.; Anheier, Helmut K. (1996): The International Classification of Nonprofit Organizations: ICNPO Rev.1, 1996. Baltimore: The Johns Hopkins Institute for Policy Studies.

Salamon, Lester M.; Haddock, Megan A.; Sokolowski, S. Wojciech; Tice, Helen

S. (2007): Measuring Civil Society and Volunteering: Initial Findings 
from Implementation of the UN Handbook on Nonprofit Institutions. Baltimore: Johns Hopkins Center for Civil Society Studies.

Salvanes, Kjell G.; Burgess, Simon; Lane, Julia (1999): Souces of Earnings Dispersion in a Linked Employer-Employee Data Set: Evidence from Norway, in: Haltiwanger, John C.; Lane, Julia I.; Spletzer, James R.; Theeuwes, Jules J.M.; Troske, Kenneth R. (Hrsg.): The Creation and Analysis of Employer-Employee Matched Data. Amsterdam: Elsevier Science B.V. 261-284.

Schafer, Joseph L. (1997): Analysis of incomplete multivariate data. London: Chapman\&Hall.

Scheffer, Judi (2002): "Dealing with Missing Data", in: Research Letters in the Information and Mathematical Sciences, 3 (1). 153-160.

Schneider, Ulrike (2008): Dimensionen der Wertschöpfung durch NonprofitOrganisationen, in: König, Joachim; Oerthel, Christian (Hrsg.): In Soziales investieren - Mehr Werte schaffen. ConSOzial 2007. München: Allitera Verlag. 117-138.

Schneider, Ulrike; Badelt, Christoph; Hagleitner, Joachim (2006): Der Nonprofit Sektor in Österreich, in: Badelt, Christoph; Meyer, Michael; Simsa, Ruth (Hrsg.): Handbuch der Nonprofit Organisationen. Strukturen und Management. Stuttgart: Schäffer Poeschel. 4. Auflage.

Schneider, Ulrike; Badelt, Christoph; Hagleitner, Joachim (2007): Der Nonprofit

Sektor in Österreich, in: Badelt, Christoph; Meyer, Michael; Simsa, Ruth

(Hrsg.): Handbuch der Nonprofit Organisationen. Strukturen und Management. Stuttgart: Schäffer Poeschel. 4. Auflage. 55-80.

Schneider, Ulrike; Trukeschitz, Birgit (2007): Öffentliche Beschaffungspolitik im Bereich sozialer und arbeitsmarktpolitischer Dienstleistungen, in: Schneider, Ulrike; Trukeschitz, Birgit (Hrsg.): Quasi-Märkte und Qualität: Die Qualität arbeitsmarktpolitischer und sozialer Dienstleistungen im Kontext öffentlicher Beschaffungspolitik. Baden-Baden: Nomos. 9-13.

Schultz, Theodore W. (1963): The Economic Value of Education. New York, London: Columbia University Press.

Schulze, Niels (2004): Applied Quantile Regression: Microeconometric, Financial, and Environmental Analyses. Dissertation. Eberhard-KarlsUniversität Tübingen.

Shackett, Joyce R.; Trapani, John M. (1987): "Earnings Differentials and Market Structure", in: The Journal of Human Resources, 22 (4). 518-531.

Simon, Hipolito (2005): "Employer wage differentials from an international perspective", in: Economics Letters, 88. 284-288.

Simsa, Ruth; Meyer, Michael (2006): Die Wiener Schule der NPO-Forschung: Wirtschatsuniversität Wien. 
Snijders, Tom A.B.; Bosker, Roel J. (1999): Multilevel Analysis. An introduction to basic and advanced multilevel modeling. London, Thousand Oaks, New Delhi: Sage Publications.

Speckbacher, Gerhard (2003): "The Economics of Performance Management in Nonprofit Organizations", in: Nonprofit Management and Leadership, 13 (3). 267-281.

Statistik Austria (2007): Statistisches Jahrbuch Österreichs 2007. Wien: Verlag Österreich $\mathrm{GmbH}$.

Statistik Austria (o.J.): Erläuterungen zur ÖNACE 2003. Wien: Statistik Austria. Steinberg, Richard (2006): Economic Theories of Nonprofit Organizations, in: Powell, Walter W.; Steinberg, Richard (Hrsg.): The Non-profit Sector. New Haven, London: Yale University Press. 117-139.

Stephan, Gesine (2001): Firmenlohndifferenziale. Eine Analyse für die Bundesrepublik Deutschland. Frankfurt, New York: Campus.

Sturman, Ted S. (1999): "Achievement Motivation and Type A Behavior as Motivational Orientations", in: Journal of Research in Personality, 33 (2). 189-207.

Tinkelman, Daniel; Mankaney, Kamini (2007): "When is Administrative Efficiency Associated With Charitable Donations?" in: Nonprofit and Voluntary Sector Quarterly, 36 (1). 41-64.

Trukeschitz, Birgit (2006): Im Dienst Sozialer Dienste. Frankfurt am Main: Peter Lang GmbH.

UN (2003): Handbook on Non-Profit Institutions in the System of National Accounts, in: United Nations Department of Economic and Social Affairs, Statistics Division (Hrsg.): Handbook of National Accounting. New York: United Nations. Series F, No. 91.

Valentinov, Vladislav (2008): "The economics of the non-distribution constraint: A critical appraisal", in: Annals of Public and Cooperative Economics, 79 (1). 35-52.

Wagner, Joachim; Schank, Thorsten; Schnabel, Claus; Addison, John T. (2006): "Works Councils, Labor Productivity and Plant Heterogeneity: First Evidence from Quantile Regressions", in: Jahrbücher für Nationalökonomie und Statistik, 226 (5). 505-518.

Warwick, Mal (2007): Guess Who's Socially Irresponsible. Nonprofits aren't as nice to their employees as you might think. Stanford Social Innovation Review. Winter 2007. 12-13.

Weichselbaumer, Doris; Winter-Ebmer, Rudolf (2005): "A Meta-Analysis of the International Gender Wage Gap", in: Journal of Economic Surveys, 19 (3). 479-511.

Weisbrod, Burton A. (1977): The Voluntary Nonprofit Sector. Lexington, Toronto: Lexington Books. 
Weisbrod, Burton A. (1983): "Nonprofit and Proprietary Sector Behavior: Wage Differentials among Lawyers", in: Journal of Labor Economics, 1 (3). 246-263.

Weisbrod, Burton A. (1988): The Nonprofit Economy. Cambridge, London: Harvard University Press.

White, Halbert (1980): "A heteroskedasticity-consistent covariance matrix estimator and a direct test für heteroskedasticity", in: Econometrica, 48 (4). 817-838.

Winter-Ebmer, Rudolf; Zweimüller, Josef (1999): "Intra-firm Wage Dispersion and Firm Performance", in: Kyklos, 52 (4). 555-572.

Wooldridge, Jeffrey M. (2002): Econometric Analysis of Cross Section and Panel Data. Cambridge, London: MIT Press.

Zauner, Alfred; Heimerl, Peter; Mayrhofer, Wolfgang; Meyer, Michael; Nachbagauer, Andreas; Praschak, Susanne (2006): Von der Subvention zum Leistungsvertrag. Neue Koordinations- und Steuerungsformen und ihre Konsequenzen für Nonprofit Organisationen - eine systemtheoretische Analyse. Bern, Stuttgart, Wien: Haupt Verlag. 


\section{Forschungsergebnisse der Wirtschaftsuniversität Wien}

Herausgeber: Wirtschaftsuniversität Wien vertreten durch a.o. Univ. Prof. Dr. Barbara Sporn

Band 1 Stefan Felder: Frequenzallokation in der Telekommunikation. Ökonomische Analyse der Vergabe von Frequenzen unter besonderer Berücksichtigung der UMTS-Auktionen. 2004.

Band 2 Thomas Haller: Marketing im liberalisierten Strommarkt. Kommunikation und Produktplanung im Privatkundenmarkt. 2005.

Band 3 Alexander Stremitzer: Agency Theory: Methodology, Analysis. A Structured Approach to Writing Contracts. 2005.

Band 4 Günther Sedlacek: Analyse der Studiendauer und des Studienabbruch-Risikos. Unter Verwendung der statistischen Methoden der Ereignisanalyse. 2004.

Band 5 Monika Knassmüller: Unternehmensleitbilder im Vergleich. Sinn- und Bedeutungsrahmen deutschsprachiger Unternehmensleitbilder - Versuch einer empirischen (Re-)Konstruktion. 2005.

Band 6 Matthias Fink: Erfolgsfaktor Selbstverpflichtung bei vertrauensbasierten Kooperationen. Mit einem empirischen Befund. 2005.

Band 7 Michael Gerhard Kraft: Ökonomie zwischen Wissenschaft und Ethik. Eine dogmenhistorische Untersuchung von Léon M.E. Walras bis Milton Friedman. 2005.

Band 8 Ingrid Zechmeister: Mental Health Care Financing in the Process of Change. Challenges and Approaches for Austria. 2005.

Band 9 Sarah Meisenberger: Strukturierte Organisationen und Wissen. 2005.

Band 10 Anne-Katrin Neyer: Multinational teams in the European Commission and the European Parliament. 2005.

Band 11 Birgit Trukeschitz: Im Dienst Sozialer Dienste. Ökonomische Analyse der Beschäftigung in sozialen Dienstleistungseinrichtungen des Nonprofit Sektors. 2006

Band 12 Marcus Kölling: Interkulturelles Wissensmanagement. Deutschland Ost und West. 2006.

Band 13 Ulrich Berger: The Economics of Two-way Interconnection. 2006.

Band 14 Susanne Guth: Interoperability of DRM Systems. Exchanging and Processing XML-based Rights Expressions. 2006.

Band 15 Bernhard Klement: Ökonomische Kriterien und Anreizmechanismen für eine effiziente Förderung von industrieller Forschung und Innovation. Mit einer empirischen Quantifizierung der Hebeleffekte von F\&E-Förderinstrumenten in Österreich. 2006.

Band 16 Markus Imgrund: Wege aus der Insolvenz. Eine Analyse der Forttührung und Sanierung insolventer Klein- und Mittelbetriebe unter besonderer Berücksichtigung des Konfigurationsansatzes. 2007.

Band 17 Nicolas Knotzer: Product Recommendations in E-Commerce Retailing Applications. 2008.

Band 18 Astrid Dickinger: Perceived Quality of Mobile Services. A Segment-Specific Analysis. 2007.

Band 19 Nadine Wiedermann-Ondrej: Hybride Finanzierungsinstrumente in der nationalen und internationalen Besteuerung der USA. 2008.

Band 20 Helmut Sorger: Entscheidungsorientiertes Risikomanagement in der Industrieunternehmung. 2008.

Band 21 Martin Rietsch: Messung und Analyse des ökonomischen Wechselkursrisikos aus Unternehmenssicht: Ein stochastischer Simulationsansatz. 2008. 
Band 22 Hans Christian Mantler: Makroökonomische Effizienz des Finanzsektors. Herleitung eines theoretischen Modells und Schătzung der Wachstumsimplikationen für die Marktwirtschaften und Transformationsőkonomien Europas. 2008.

Band 23 Youri Tacoun: La théorie de la valeur de Christian von Ehrenfels. 2008.

Band 24 Monika Koller: Longitudinale Betrachtung der Kognitiven Dissonanz. Eine Tagebuchstudie zur Reiseentscheidung. 2008.

Band 25 Marcus Scheiblecker: The Austrian Business Cycle in the European Context. 2008.

Band 26 Aida Numic: Multinational Teams in European and American Companies. 2008.

Band 27 Ulrike Bauernfeind: User Satisfaction with Personalised Internet Applications. 2008.

Band 28 Reinhold Schodl: Systematische Analyse und Bewertung komplexer Supply Chain Prozesse bei dynamischer Festlegung des Auftragsentkopplungspunkts. 2008.

Band 29 Bianca Gusenbauer: ÖHfentlich-private Finanzierung von Infrastruktur in Entwicklungslăndern und deren Beitrag zur Armutsreduktion. Fallstudien in Vietnam und auf den Philippinen. 2009.

Band 30 Elisabeth Salomon: Hybrides Management in sino-österreichischen Joint Ventures in China aus österreichischer Perspektive. 2009.

Band 31 Katharina Mader: Gender Budgeting: Ein emanzipatorisches, finanzpolitisches und demokratiepolitisches Instrument. 2009.

Band 32 Michael Weber: Die Generierung von Empfehlungen für zwischenbetriebliche Transaktionen als gesamtwirtschaftliche Infrastrukturleistung. 2010.

Band 33 Lisa Gimpl-Heersink: Joint Pricing and Inventory Control under Reference Price Effects. 2009.

Band 34 Stefan A. Zopf: Analyse der Potentiale und Effekte des Ingredient Brandings und dessen Einfluss auf die Markenstärke. 2009.

Band 35 Dagmar Kiefer: Multicultural Work in Five United Nations Organisations. An Austrian Perspective. 2009.

Band 36 Gottfried Gruber: Multichannel Management. A Normative Model Towards Optimality. 2009.

Band 37 Rainer Quante: Management of Stochastic Demand in Make-to-Stock Manufacturing. 2009.

Band 38 Franz F. Eiffe: Auf den Spuren von Amartya Sen. Zur theoriegeschichtlichen Genese des Capability-Ansatzes und seinem Beitrag zur Armutsanalyse in der EU. 2010.

Band 39 Astrid Haider: Die Lohnhöhe und Lohnstreuung im Nonprofit-Sektor. Eine quantitative Analyse anhand österreichischer Arbeitnehmer-Arbeitgeber-Daten. 2010.

www.peterlang.de 


\section{Im Dienst Sozialer Dienste \\ Ökonomische Analyse der Beschäftigung in sozialen Dienstleistungseinrichtungen des Nonprofit Sektors}

Frankfurt am Main, Berlin, Bern, Bruxelles, New York, Oxford, Wien, 2006. 304 S., 60 Tab., 8 Graf.

ISBN 978-3-631-53929-3 · br. $€ 54,70$ *

In der Versorgung von benachteiligten Menschen mit sozialen Dienstleistungen spielen Nonprofit Organisationen eine wesentliche Rolle. Dieses Buch befasst sich zunächst aus ökonomischer Sicht mit den charakteristischen Eigenschaften sozialer Dienstleistungen und fokussiert im Anschluss daran auf die Beschäftigung in sozialen Dienstleistungseinrichtungen des Nonprofit Sektors. Ein Set von zentralen Forschungsfragen leitet diese Studie: Aus theoretischer Sicht interessierten die ökonomischen Bestimmungsgründe und Besonderheiten des Arbeitskräfteeinsatzes in diesen Einrichtungen. Der empirische Teil gibt einen detaillierten Einblick in die Methodik der Erhebung und in die Ergebnisse der Analysen zum Beschäftigungsvolumen und zur Beschäftigungsstruktur in Sozialen Diensten des österreichischen Nonprofit Sektors.

Aus dem Inhalt: Eigenschaften sozialer Dienstleistungen aus ökonomischer Sicht · Soziale Dienstleistungen in nationalen und internationalen Klassifikationen wirtschaftlicher Tätigkeiten - Nonprofit Organisationen als Anbieterinnen sozialer Dienstleistungen - Theoretische Grundlagen der Beschäftigung in Sozialen Diensten des Nonprofit Sektors - Methodische Grundlagen der empirischen Erhebung der Beschäftigung in Sozialen Diensten des Nonprofit Sektors · Stichprobenbeschreibung · Soziale Dienste des Nonprofit Sektors 2002 . Hochrechnungen zu Volumen und Struktur des Arbeitskräfteeinsatzes in Sozialen Diensten des Nonprofit Sektors allgemein sowie nach Angebotsschwerpunkten

Frankfurt am Main - Berlin - Bern - Bruxelles - New York - Oxford - Wien

Auslieferung: Verlag Peter Lang AG

Moosstr. 1, $\mathrm{CH}-2542$ Pieterlen

Telefax 0041 (0) $32 / 3761727$

*inklusive der in Deutschland gültigen Mehrwertsteuer

Preisänderungen vorbehalten

Homepage http://www.peterlang.de 